\title{
Cell death and synaptic remodelling as a consequence of perinatal asphyxia : implications of hypothermia
}

Citation for published version (APA):

van de Berg, W. D. J. (2003). Cell death and synaptic remodelling as a consequence of perinatal asphyxia : implications of hypothermia. [Doctoral Thesis, Maastricht University]. Universiteit Maastricht. https://doi.org/10.26481/dis.20030612wb

Document status and date:

Published: 01/01/2003

DOI:

10.26481/dis.20030612wb

Document Version:

Publisher's PDF, also known as Version of record

\section{Please check the document version of this publication:}

- A submitted manuscript is the version of the article upon submission and before peer-review. There can be important differences between the submitted version and the official published version of record. People interested in the research are advised to contact the author for the final version of the publication, or visit the DOI to the publisher's website.

- The final author version and the galley proof are versions of the publication after peer review.

- The final published version features the final layout of the paper including the volume, issue and page numbers.

Link to publication

\footnotetext{
General rights rights.

- You may freely distribute the URL identifying the publication in the public portal. please follow below link for the End User Agreement:

www.umlib.nl/taverne-license

Take down policy

If you believe that this document breaches copyright please contact us at:

repository@maastrichtuniversity.nl

providing details and we will investigate your claim.
}

Copyright and moral rights for the publications made accessible in the public portal are retained by the authors and/or other copyright owners and it is a condition of accessing publications that users recognise and abide by the legal requirements associated with these

- Users may download and print one copy of any publication from the public portal for the purpose of private study or research.

- You may not further distribute the material or use it for any profit-making activity or commercial gain

If the publication is distributed under the terms of Article $25 \mathrm{fa}$ of the Dutch Copyright Act, indicated by the "Taverne" license above, 


\section{Cell death and synaptic remodelling as a consequence of perinatal asphyxia}

Implications of hypothermia

Wilma Dorethea Johanna van de Berg 
W.D.J. van de Berg, Maastricht 2003

Cell death and synaptic remodelling as a consequence of perinatal asphyxia Implications of hypothermia.

Thesis with summary in English and Dutch

ISBN: $90-9016940-7$

Author: W.D.J. van de Berg

Cover: Angeline Taal, Artee Grafische Vorming

Lay-out: W.D.J. van de Berg

Productions: Datawyse I Universitaire Pers Maastricht 


\title{
Cell death and synaptic remodelling as a consequence of perinatal asphyxia
}

\section{Implications of hypothermia}

\author{
PROEFSCHRIFT
}

ter verkrijging van de graad van doctor

aan de Universiteit Maastricht, op gezag van de Rector Magnificus, Prof. dr. A.C. Nieuwenhuijzen Kruseman, volgens het besluit van het College van Decanen, in het openbaar te verdedigen op donderdag 12 juni 2003 om 12:00 uur

door

Wilma Dorethea Johanna van de Berg geboren op 1 maart 1974 te St. Geertruid 


\section{Promotores}

Prof. dr. C.E. Blanco

Prof. dr. H.W.M. Steinbusch

\section{Beoordelingscommissie}

Prof. dr. J.S.H. Vles (voorzitter)

Dr. T.H. Hasaart

Prof. dr. H. Korr (RWTH University of Aachen, Germany)

Dr. C. Mallard (Göteborg University, Sweden)

Prof. dr. F.C. Ramaekers

The study presented in this thesis is a research project of the department of paediatrics of the Academic Hospital Maastricht and the University Maastricht and was performed at research institute Growth and Development (GROW) and the research institute Brain and Behaviour, University of Maastricht, The Netherlands.

The publication of this thesis was supported by (in alphabetical order) Abbott BV, Charles River NL, Datex-Ohmeda BV, Microbrightfield Europe, Paes Nederland BV, Van Leersum Fund of the Royal Dutch Academy of Sciences and 'Stichting Bevordering Kindergeneeskunde'. 


\section{Contents}

Prologue

\section{Chapter 1}

General introduction

Chapter 2

Use of cryostat sections from snap-frozen nervous tissue for combining stereological estimates with histological, cellular, or molecular analyses on adjacent sections

$J$ Chem Neuroanatomy 2000;20:21-29

\section{Chapter 3}

Perinatal asphyxia induced neuron loss by apoptosis in the neonatal rat striatum: a combined TUNEL and stereological study

Experimental Neurology 2002:174:29-36

\section{Chapter 4}

Qualitative and quantitative analysis of the ontogeny of apoptotic cell death during postnatal development after birth asphyxia

Experimental Neurology, in press

\section{Chapter 5}

Perinatal asphyxia increases DAF-2/NO fluorescence during postnatal development in the striatum, but not cerebellum

Eur J Neuroscience, in press

\section{Chapter 6}

Impact of perinatal asphyxia on the GABAergic and molor system Neuroscience 2003;117:83-96

\section{Chapter 7}

Regional specificity in neuronal and synaptic loss after perinatal asphyxia in the rat brain

Brain Research, in press

\section{Chapter 8}

Perinatal asphyxia results in changes in presynaptic bouton number in striatum and cerebral cortex: a stereological and behavioural analysis $J$ Chem Neuroanatomy 2000:20:71-82 
Chapter 9

183

Hypothermia prevents cytoplasmic stress gene response induced by perinatal asphyxia

Chapter 10

General discussion

Summary

204

Samenvatting

209

Curriculum vitae

213

Publications

214

Dankwoord

216 


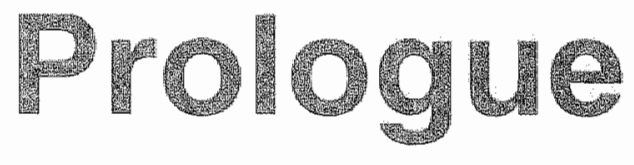




\section{Cell death and synaptic remodelling as a consequence of perinatal asphyxia. Implications of hypothermia.}

This thesis will focus on perinatal asphyxia and its consequences for central nervous system (CNS) development. It has been estimated that once an hour an infant is born with cerebral palsy and that every $5 \mathrm{~min}$, an infant is born with mental retardation. Although only a small percentage of these infants have suffered from perinatal asphyxia [13,14], this group is particularly interesting as their outcome might be improved considerably by appropriate therapy.

Perinatal asphyxia causes cell death within a critical period in CNS development $[4,8,15]$. Mitochondrial and endoplasmatic reticulum failure, as a consequence of oxygen and energy deprivation, play an important role in the rate and morphological type of cell death following perinatal asphyxia $[9,10,12,17]$. Immediate cell death after the insult occurs mainly by necrosis and starts before treatment can be given. Therapeutic intervention has to be aimed at inhibition of delayed neuronal death (apoptosis) and stimulation of regeneration processes. One of the most potential and promising interventions is mild hypothermia $[1,6,19,20]$. Post-asphyxia hypothermia may be able to decrease the amount of cell damage by decreasing the metabolic rate (energy consumption) and subsequently the production of endogenous cell damaging agents, such as nitric oxide, stress response genes, activated caspases etc $[5,16,18]$. The clinical effectiveness of hypothermia is still largely unknown, but there are currently three clinical trials addressing the issue.

The neurological outcome after perinatal asphyxia is strongly dependent on neuronal and synaptic plasticity, which is the ability of neuronal tissue to change or adapt. Several compensatory mechanisms are described in the CNS after injury: regenerative sprouting, pre- or postsynaptic changes, network rearrangement, synaptogenesis or neurogenesis. However, the processes that facilitate function recovery after hypoxic-ischemic injury are not fully understood. Insight in these processes can be a great asset to treatment strategies.

In this thesis, cell death and synaptic remodelling were studied on different levels: 1) on a cellular level, 2) neurochemical and neuroanatomical level, 3) behavioural level. At the cellular level, nitric oxide production, the induction of stress genes and the activation of caspases were assessed at different times during the first two weeks after perinatal asphyxia in the rat. Functional outcome (behaviour) was studied using locomotor (open field, grip task, footprint task) and memory tasks (Morris water maze escape task). The behavioural measurements were correlated to neurochemical (GABA) and neuroanatomical (total number of neurons and synapses within brain regions, vollume of brain regions) parameters. These data were used to gain insight into adaptation of the brain during physiological (i.e. CNS development) and pathological (i.e. neurodegeneration) conditions and explore possible avenue of treatment. 


\section{Aims and outline of the thesis}

The current state of knowledge of perinatal brain damage and its consequences originates primarily from animal studies. Experimental animal models generally aim at investigating the effects of a single factor in order to assure specificity with regard to the source initiating the pathophysiological response. Perinatal asphyxia, however, is a global hypoxic-ischemic insult, which is accompanied by altered blood flow, cellular stress and by CNS plasticity. Consequently, a good animal model of perinatal asphyxia should mimic the multiplicity of causes acting at both cellular and anatomical levels. In our studies, we used a non-invasive animal model that induces intrauterine global asphyxia at the time of birth. This animal model induces cellular damage within the basal ganglia, hippocampus, cerebellum and spinal cord $[2,3]$. Furthermore, it is shown that this global asphyxia paradigm can induce long-lasting behaviour deficits $[7,11]$.

This thesis focuses on cell death and synaptic remodelling as a consequence of perinatal asphyxia and the usefulness of hypothermia as a therapeutic strategy. The following aims were formulated:

1. Characterization of a non-invasive animal model of global asphyxia at birth.

2. To gain insight into the cellular processes induced by perinatal asphyxia, which lead to cell death.

3. To gain insight into the ability of the CNS to compensate for or adapt to cellular damage during $\mathrm{CNS}$ development.

4. To study the long-term consequences of perinatal asphyxia in relation to the aging process.

5. To gain insight into the effectiveness and possible neuroprotection of post-asphyxia hypothermia.

In the first chapter of this thesis, an overview of the literature is given concerning experimental models for perinatal asphyxia, structural and functional consequences of perinatal asphyxia and potential therapies. The purpose of this review was to evaluate the advantages and disadvanges of different experimental models for perinatal asphyxia. Moreover, to investigate whether these models can resemble the clinical symptoms of perinatal asphyxia. First, mechanisms of cell death during normal development are compared with mechanisms of cell death after perinatal asphyxia. Secondly, brain structures and neurotransmitter systems involved in locomotor behaviour and cognitive performance are discussed as well as the behavioural deficits described in the different experimental models. Finally, we evaluate which potential therapy could be interesting for clinical use.

First, we present a study in which methodological issues concerning quantification of total neuronal numbers within the brain are discussed (chapter 2). The following chapters (chapter 3-9) of this thesis describe the experiments with the global asphyxia model at term in the rat. This model is used to study both short-term and long-term consequences of the asphyctic insult. The first four chapters address the characterisation of the amount of cell damage and mechanism of cell damage within several brain regions after the asphyctic insult. The time 
course of cell death after perinatal asphyxia is compared with the time course of apoptosis during normal development (chapter 3 and 4). Furthermore, we studied whether caspase-3 activity, NO production and stress response genes play an important role in the mechanism of cell death in this animal model for perinatal asphyxia (chapter 4,5 and 9).

Next, we investigated whether behavioural deficits following this global asphyctic insult at term were related to loss of GABAergic neurons in the striatum (chapter 6) and/or loss of presynaptic boutons (chapter 7) within the striatum, hippocampus and/or cerebellum. Thereafter, the relation between cognitive performance (short-term and long-term memory performance) and synaptic plasticity was studied at 22 months after the perinatal asphyctic insult (chapter 8). These experiments were designed to investigate whether global asphyxia at term could have long-lasting behavioural consequences related to neurodegenerative diseases, such as dementia. In addition, we evaluated whether post-insult hypothermia was able to prevent the cellular stress response (chapter 9). Finally, in chapter 10 a general discussion of the combined results presented in this thesis is given. 


\section{References}

[1] Battin, M.R., Dezoete, J.A., Gunn, T.R., Gluckman, P.D. and Gunn, A.J. 200 H. Neurodevelopmental outcome of infants treated with head cooling and mild hypohermia after perinatal aspliyxia. Pediatrics 107: 480-4

[2] de Louw, A.J., de Vente, J., Steinbusch, H.P., Gavilanes, A.W., Steinbusch, H.W., Blanco, C.E., Troost, J. and Wles, J.S. 2002. Apoptosis in the rat spinal cord during posinatal development; the effect of perinatal asphyxia on programmed cell death. Neuroscience $112: 75 \|-8$

[3] Dell'Anna, E., Chen, Y., Engidawork, E., Andersson, K., Lubec, G., Luthman, J. and HerreraMarschitz, M. 1997. Delayed neuronal death following perinatal asphyxia in rat. Exp Brain Res 115 $105-15$.

[4] Edwards, A.D., Yue, X., Cox, P., Hope, P.L., Azzopardi, D.V., Squier, M.V. and Mehmet, H. 1997 Apoptosis in the brains of infants suffering intrauterine cerebral injury. Pediatr Res 42: 684-9.

[5] Fukuda, H., Tomimatsu, T., Watanabe, N., Mu, J.W. Kohzuki, M., Endo, M., Fujii, E., Kanzaki, T. and Murata, Y. 200॥. Post-ischemic hypothermia blocks caspase-3 activation in the newborn rat brain after hypoxia-ischemia. Brain Res 910:187-91.

[6] Gunn, A.J. 2000. Cerebral hypothermia for prevention of brain injury following perinatal asphyxia. Curr Opin Pediatr 12: $111-5$.

[7] Hoeger, H., Engelmann, M., Bernert, G., Seidl, R., Bubna-Littitz, H., Mosgoeller, W., Lubec, B. and Lubec, G. 2000. Long term neurological and behavioral effects off graded perinatal asphyxia in the rat. Life Sci 66: 947-62.

[8] Inder, T.E. and Volpe, J.J. 2000. Mechanisms of perinatal brain injury. Semin Neonatol 5:3-16.

[9] Kroemer, G., Zanzami, N. and Susin, S.A. 1997. Mitochondrial control of apoptosis. Immunol Today $18: 44-51$.

[10] Leist, M. and Nicotera, P. 1997. The shape of cell death. Biochem Biophys Res Commun 236: 1-9.

[11] Loidl, C.F., Gavilanes, A.W., Van Dijk, E.H., Vreuls, W., Blokland, A., Vles, J.S., Steinbusch, H.W. and Blanco, C.E. 2000. Effects of hypothermia and gender on survival and behavior after perinatal asphyxia in rats. Physiol Behav 68: 263-9.

[12] Mishra, O.P. and Delivoria-Papadopoulos, M. 1999. Cellular mechanisms of hypoxic injury in the developing brain. Brain Res Bull 48: 233-8.

[13] Nelson, K.B. and Grether, J.K. 1999. Causes of cerebral palsy. Curr Opin Pediatr 11: 487-91.

[14] Pschirrer, E.R. and Yeomans, E.R. 2000. Does asphyxia cause cerebral palsy? Semin Perinatol 24: 215-20.

[15] Rossiter, J.P., Anderson, L.L., Yang, F. and Cole, G.M. 2002. Caspase-3 activation and caspase-like proteolytic activity in human perinatal hypoxic-ischemic brain injury. Acta Neuropathol (Berl) 103 $66-73$.

[16] Tomimatsu, T., Fukuda, H., Endo, M., Watanabe, N., Mu, J., Kohzuki, M., Fujii, E., Kanzaki, T. and Murata, Y. 2001. Effects of hypothermia on neonatal hypoxic-ischemic brain injury in the rat: phosphorylation of Akt, activation of caspase-3-like protease. Neurosci Lett 312:21-4.

[17] Volpe, J.J. 2001. Perinatal brain injury: from pathogenesis to neuroprotection. Ment Retard Dev Disabil Res Rev 7: 56-64.

[18] Wagner, B.P., Nedelcw, J. and Martin, E. 2002. Delayed postisclemic hypothermia improves longterm behavioral outcome after cerebral hypoxia-ischemia in neonatal rats. Pediatr Res $51: 354-60$.

[19] Wagner, C.L., Eicher, D.J., Katikaneni, L.D., Barbosa, E. and Holden, K.R. 1999. The use of hypothermia: a role in the treatment of neonatal asphyxia? Pediatr Neurol 21:429-43.

[20] Willarms, G.D., Dardzinski, B.J., Buckalew, A.R. and Smith, M.B. 1997. Modest hypothennia preserves cerebral energy metabolism during hypoxia-ischemia and correlates with brain damage: a $3 \mathrm{l} P$ nuclear magnetic resonance study in unanesthetized neonatal rats. Pediatr Res 42: 700-8. 
$-12-$

<゙- 


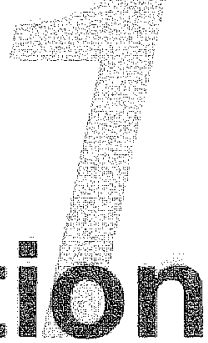

General introduction 


\section{Introduction}

Asphyxia before, during or after bith is a common cause of perinatal mortality and neurological morbidity, with a prevalence of 1.5 to 2.5 per 1000 live term infants [101]. Perinatal asphyxia is a condition defined as a period of global hypoxia and ischemia around birth. The Greek word 'asphyxia' reflects the clinical signs of an infant without visible breathing or heart action as a result of lack of oxygen [38].

Asphyxia occurs when there is a disturbance in gas exchange between the foetus and mother during pregnancy or labour. During periods of asplayxia, the foetus reduces its metabolic rate and utilizes anaerobic glycolysis to meet its energy needs. The subsequent formation of lactic acid results in a decrease of foetall blood $\mathrm{pH}$ (acidosis) $[235,236]$. The foetus is equipped with an impressive of physiologic, pharmacological and metabolic adaptive systems to enable it to survive the hypoxic-ischemic insult. Brain injury only occurs when the asphyxia is severe enough to impair cerebral blood flow. Within the brain, blood flow during asphyxia is redirected to the brainstem at the expense of higher cerebral structures such as the cortex. Furthermore, several cases were reported with selective vulnerability of basal ganglia [179], hippocampus [170,199], cerebellum and spinal cord $[49,203]$.

Clinical features observed in the neonate after perinatal asphyxia include seizures, abnormal respiratory patterns, movement disorders and impaired suck, swallow and feeding behaviour and multiple organ failure [38]. Some infants recover, whereas others develop permanent brain damage resulting in mental or motor disabilities, such as hyperactivity and attention deficit disorders, epilepsy, cerebral palsy or spasticity [128]. The prognosis of the infant is mainly dependent on the duration of the asphyxia. The risk for motor abnormalities, such as cerebral palsy, has for example been directly correlated to the duration of severely depressed Apgar scores and clinical signs of seizures, hypotonia, or multiorgan system dysfunction \186].

Perinatal asphyxia and its consequences are studied in animals by inducing either focal or global hypoxia-ischemia. Focal hypoxia-ischemia affects a specific region and is characterized by an infarct within this region (lesion site). It is induced by an occlusion or vascular interruption followed by anoxia/hypoxia. Global hypoxia-ischemia occurs when all blood supply to the brain is interrupted. This can be induced intrauterine by clamping or interuption of the vessels during the prenatal of perinatal period. In the next paragraphs, two different animal models for perinatal asphyxia are discussed: the Rice-Vannucci model (unillateral carotid ligation followed by exposure to hypoxia) [196] and the global asphyxia model (intrauterine hypoxia-ischemia) [15]. The term asphyxia is used in this thesis when manipulating the concentration of inspired gas mixtures or ventilator settings, preventing breathing, or reducing uterine perfusion, produced hypoxia-ischemia. The term "hypoxiaischemia' is used when cerebral ischemia was produced by unilateral or bilaterall ligation or occlusion of the conmon carotid arteries and followed by hypoxia (reducing oxygen concentration). 


\section{Experimental models of perinatal asphyxia}

Much of our current knowledge concerning the pathophysiology of perinatal asphyxia has evolved from animal studies, using piglets, sheep or rodents. The rat is most frequentlly used in experimental studies, as they are easily handled and suitable for use in models studying acute and short-term consequences, as well as long-term effects. Another advantage of using rats as animal model is the large amount of literature already available on baseline neurochemistry, physiology and neurological methods of behavioural testing. A clear disadvantage of the rat as a model to study perinatal asphyxia is the uncertainty whether or not rat brain maturation is comparable to human brain maturation. Compared to the human, the rat is bonn very prematurely. Comparing the brain growth, it is suggested that the maturity levels of the brain of a 10-day-old rat approximates to the developmental stage of a fullterm human infant's brain $[59,60,176]$. However, brain maturation in both rat and human has not been established yet for many critical events during brain development, such as differentiation, migration, myelination, protein synthesis etc. The postnatal rate of maturation in rats is also more rapid compared to infants [60]. Finally, a disadvantage of using rats is the relatively small body size, consequently multiple organ function is difficult to monitor [198].

\section{The Rice-Vannucci model}

Over the last decade, many researchers have used a model of hypoxic-ischemic brain damage in the 7-day-old rats to study consequences of perinatal asphyxia. In this model, hypoxiaischemia is produced by unilateral carotid artery ligation followed by exposure to hypoxia by means of reduction of oxygen concentration ( $8 \%$ oxygen) for $1-3 \mathrm{hrs}$. This animal model is known as the Rice-Vanmucci neonatal adaptation [196] of the Levine procedure in the adult rat [145]. The model has been modified by several researchers to reduce the extent of the brain lesion and the variability of neuronal loss, which depends mainly on the length of hypoxia and the time interval between the ischemic and hypoxic insult. Brain damage in this neonatal hypoxiaischemia model is restricted to one hemisphere ipsilateral to the occluded artery and is observed within the cortex, the subcortical and periventricular white matter, the striatum, and the hippocampus. In this model, the hypoxic-ischemic insult leads to necrotic cell death within hours after the insult accompanied by oedema and delayed apoptotic cell death.

The Rice-Vannucci model was modified by Hattori et al. by ligating both carotid arteries and subjecting the rat pups to 60 min of hypoxia ( $8 \%$ oxygen) [95]. This procedure consistently produced severe neocortical infarction and selective neuronal death in the granule cell layer of the dentate gyrus $[188,204,243]$. Other researchers studied the developmental changes in the sensitivity of neonatal rat brain to hypoxia-ischemia by applying the Rice-Vannucci model at different time points up to 25 days after birth $[16,76,82,96,100,123,208,246]$. The extent of injury was variable, ranging from focal neuronal death to massive cortical infarction. Application of hypoxia-ischemia at younger ages (1,3 or 5 dlays after birth) [246] led to a decrease of the size of the infarct as compared to the older ages $(7,14,21$ days after birth) $[16,82,208]$. 
The Rice-Vannucci model has proven to be an excellent tool to study physiological, biochemical and molecular mechanisms of perinatal hypoxic-ischemic brain damage. In addition, this model has been used extensively to study physiological and therapeutic variables $[1,178,226,240]$, which may be beneficial to the developing brain undergoing hypoxia-ischemia. However, the major concern with regards to the suitability of this model to study perinatal asphyxia is the invasive nature of the Rice-Vannucci model. Rather than inducing global asphyxia, in which multiple organ failure is one of the key events, this model creates an infarct in a certain area. Therefore, this model could be considered as an animal model of perinatal stroke.

\section{Global asphyxia at term}

Bjelke and co-workers introduced a non-invasive rat model for studying short-term and longterm consequences of perinatal asphyxia in 1991. In this model, global asphyxia was induced during delivery, mimicking the conditions resulting in asphyxia during human labour $[15,43,44]$. Asphyxia was induced in pups by removing the uterus horns of pregnant dams at the last day of gestation by hysterectomy (see Figure 1). The uterus horns, still containing the foetuses, were then placed in a water bath at $37^{\circ} \mathrm{C}$ for various periods of time. The severity of the brain damage in this model is dependent on the duration of the asphyxia, as well as the temperature of the water. At $37^{\circ} \mathrm{C}, 100 \%$ survival was observed up to a 16 min asphyctic period, and asphyctic periods longer than $21 \mathrm{~min}$ were inevitably associated with $100 \%$ mortality. However, $100 \%$ survival was still observed for up to a $30 \mathrm{~min}$ asphyctic period at $30^{\circ} \mathrm{C}$ and up to a 100 min asphyctic period at $15^{\circ} \mathrm{C}[152,154]$. Rats surviving asphyctic periods longer than $19 \mathrm{~min}$ at $37^{\circ} \mathrm{C}$ showed chronic alterations in the release patterns of neurotransmitters, morphological alterations in the striatum and cortex and a decrease in spontaneous motor activity in later life $[43,54,55,102]$. Further, apoptotic cell death, but not necrotic cell death, was found in hippocampus, striatum and cerebellum after asphyxia [54].

Although brain maturation of the rat at the time of birth is different from human brain maturation, this model seems to be very suitable to study the consequences of global asphyxia, as the pattern of brain damage is similar to that seen in neonatal infants. Moreover, the model is easy to perform without the use of any anaesthetics or invasive surgical procedures. Disadvantages of the model include the high mortality in the group exposed to 19-20 min of asphyxia $(40-80 \%)$ and the high variability in the outcome of the pups. Furthermore, the rat pup is still very small at P0, consequently difficult to monitor and to handle, e.g. for
protective treatments. 


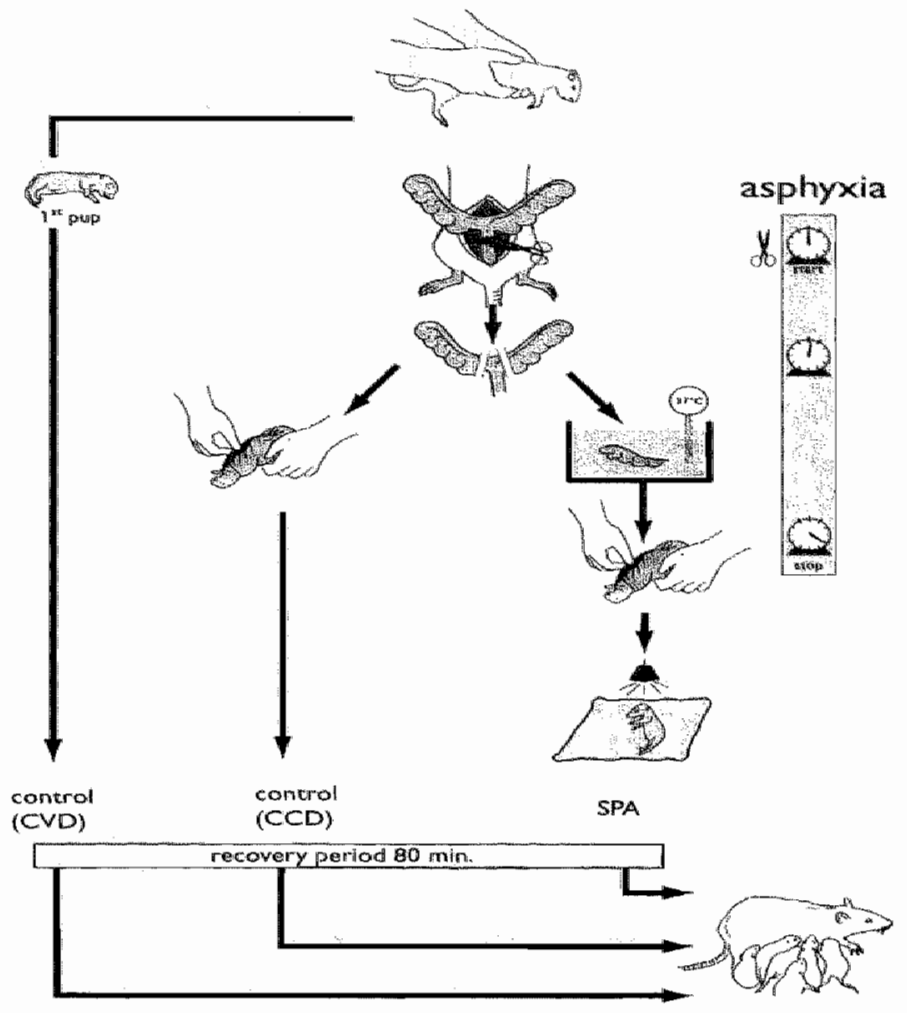

Figure 1. Rat model for perinatal asphyxia. Asphyxia was iduced in pups by removing the uterus horns of the pregnant dam at the last day of gestation by hysterectomy. CVD: control vaginall delivery; CCD: control caesarean delivery; SPA: severe perinatal asphyxia.

\section{Cell death during normal development of the CNS}

\section{Cell death}

Cell death is frequently classified according to morphological or biochemical features into two types: necrosis and apoptosis [118,119]. Necrosis refers to a passive and acute process characterized by swelling, disturbance of osmotic balance, cytolysis and tissue inflammation. The basic mechanism of necrosis is thought to be a loss of control over cell volume, related. to changes in the permeability of the cell membrane. The affected membrane rapidly loses its' ion-pumping capacity, resulting in an osmotic shock and the development of intracellular acidosis. The point of no return is reached with irreversible damage to mitochondrial structure and function [19]. 
Apoptosis refers to a selective, programmed type of cell death characterized by cell and nuclear shrinkage, chromatin condensation and nuclear fragmentation [245]. The term apoptosis is derived from the Greek word meaning "falling off" (as leaves do in autumn) to describe this natural, timely death of cells [118]. Endogenous and/or exogenous agents can initiate apoptosis in many ways. All different path ways lead eventually to the activation of a family of proteolytic enzymes called caspases [97]. A short overview of different pathways that have been identified will be given in the next paragraph.

\section{Molecular regulation of apoptosis}

The apoptotic program can be divided into three distinct phases: initiation, commitment of propagation, and execution phase [163]. The initiation phase is defined as the stage in which the cell receives a trigger to commit to the cell death process or lost a survival factor. During the commitment phase, multiple signalling pathways are activated leading to activation of caspases. This phase is controlled by mitochondrial mechanisms in which the Bcl-2 family of proteins plays an important role. The execution phase involves multiple pathways that lead to the critical features of apoptosis, such as DNA laddering, lamin disassembly and chromatin condensation $[97,254]$.

Mitochondria play an important role in the decision on the rate [127] and shape [137] of cell death. The mitochondria act as a calcium buffer, taking up the calcium when cytoplasmic levels rise. Increase in mitochondrial calcium levels can lead to opening of permeability transition pores (non-specific mitochondrial membrane pores), leading to depolarisation of the mitochondrial membrane, mitochondrial dysfunction and swelling $[83,194]$. The intermembrane space of the mitochondria contains cytochrome $\mathrm{C}$, certain pro-caspases, adenylate kinase 2 and apoptosis-inducing factor (AIF) [149,250]. Permeabilisation of the outer membrane by the onset of apoptosis results in the release of these factors. Cytochrome $C$ is a nuclear DNA encoded protein involved in electron transport during oxidative phosphorylation. Cytochrome c can bind to Apaf-1 (apoptotic protease-activating factor) and pro-caspase 9 to form an apoptosome [149]. Pro-caspase 9 is then cleaved into active caspase 9 , which can further activate pro-caspase 3 resulting in active caspase 3 , an active effector caspase and cell death [256].

The Bcl-2 family of proteins play a crucial role in signalling transduction pathways leading to apoptosis. This family consists of approximately 15 members and can be divided in antiapoptotic (Bcl-2, Bcl-XL, Bcl-W, Mcl-1) and pro-apoptotic (Bax, Bak, Bok, Bid, Bad, BclXS, Bim, Bik, Blk, Hrk) proteins [2, 192]. The anti-apoptotic family members all possess Bcl2 homology domains (BH) 1 and 2 , and some contain additional BH3 and BH4 domains. The pro-apoptotic family members all possess a $\mathrm{BH} 3$ domain, but can be subdivided by the presence of absence of $\mathrm{BH} 1$ and $\mathrm{BH} 2$ domains [2]. Various members of the Bcl-2 family control the membrane permeabilisation of mitochondria and the release of cytochrome C or AIF. Some members, such as Bcl-2 or Bcl-xL, act as anti-apoptotic factors by preventing activation of executioner caspases. Bel-2 homologues can interact directly with caspases or other apoptosisinhibiting proteins such as Bag-1 [218] and Raf-1 [242]. Active Raf-1 can protect cells from 
apoptosis and inactivate $\mathrm{BAD}$, a proapoptotic $\mathrm{Bcl}-2$ homologue. $\mathrm{Bcl}-2$ can also bind to $\mathrm{Apaf}-$ 1 , enabling Apaf- 1 to activate caspases. Bcl-2 and Bcl-xL are able to form membrane channels for specific ions and regulate the permeability of intracellular membranes [193]. In this way, $\mathrm{Bcl}-2$ and $\mathrm{Bcl}-\mathrm{xL}$ can prevent the release of cytochrome $\mathrm{C}$ from mitochondria. In contrast, overexpression of the Bax, Bak or Bid stimulates both the cytochrome $\mathrm{C}$ release and caspase activation. Bax, Bad, Bak, Bik, Bid, Bim, Hrk, Bcl-xS, can block the anti-apoptotic effects of $\mathrm{Bcl}-2$ or $\mathrm{Bcl}-\mathrm{xL}$. These family members promote activation of caspases among others by dissociating complexes of $\mathrm{Bcl}-2$ or $\mathrm{Bcl}-\mathrm{xL}$ with Apaf-1.

The signalling pathways initiating and controlling apoptosis lead to activation of caspases. All known caspases possess an active-site cysteine, and cleave substrates after aspartic acid residues. There are at least 14 different caspases [225], initiator $(1,2,4,5,8,10-14)$ and executioner $(3,6,7,9)$ caspases, which have been subdivided into subfamilies based on their substrate preference, extent of sequence identity and structural similarities. For instance, caspases with caspase-activating recruitment domains (CARDs), which includes caspase-1, $2,-4,-5,-9,-11$ and -12 , are probably activated by the cytochrome C/Apaf-1/caspase-9 complex. In contrary, caspase- 8 and -10 are activated by interaction between their so-called 'death-domain' and the intracellular domain of 'death receptors'. These 'death receptors' allow apoptosis to be initated by extracellular factors, including TNF-alpha, the TNF-alpha-related apoptosis inducing ligand (TRIAL) and Fas-ligand that can bind to the TNF-receptor, the TRAILreceptors and Fas, respectively. Finally, recent data have provided evidence that certain caspases, such as caspase-11 and -12, are activated only under pathological conditions [168].

Proteolytic cleavage by caspases can lead to loss of biological activity of the substrate by cleaving off a negative regulatory domain or by inactivating a regulatory subunit. Close to 100 caspase substrates have been reported over the years, many of which are not relevant substrates but more 'innocent bystanders'. Willy and co-workers described in 1980 that activation of a DNA ladder nuclease (now known as caspase-activated DNase, or CAD), which is responsible for cutting the genomic DNA between nucleosomes to generate DNA fragments [201]. Activation of CAD is induced by caspase-3 mediated cleavage of an inhibitory subunit of CAD leading to activation of its catalytic activity. Cleavage and inactivation of nuclear lamins by caspases is responsible for nuclear shrinking and budding $[26,191]$. Cleavage of cytoskeletal proteins such as fodrin and gelsolin [126] is required for loss of overall cell shape. Caspase-mediated cleavage of PAK2, a member of the p21activated kinase family, seems to be responsible for the blebbing observed in apoptotic cells $[97,200]$. These characteristic features of apoptosis have been used extensively as markers of apoptotic cell death.

The above-described signalling pathways inducing cell death are all dependent on the activation of caspases. However, there are other cell death pathways described in the CNS that are caspase-independent, such as the Bax pathway $[79,85,97,1.15]$, release of apoptosis inducing factor (AIF) from the mitochondrial membrane [214] or activation of other proteases that mediate apoptotic cell death. Bax and Bax-like proteins can, after activation, release cytochrome C, AlF and other pro-apoptotic molecules. AlF can activate a nuclear DNase. Proteases that can induce apoptosis in a caspase-independent manner include calpains [212] 
and serine proteases [104]. Little is known about the molecular and biochemical changes induced by these factors in the CNS.

\section{Detection of cell death in the CNS}

Cell death in the immature brain can be studied in many ways using morphological and biochemical markers. Essential requirements for apoptosis detection techniques in the central nervous system include high sensitivity for apoptotic cells, the ability to differentiate between apoptotic and necrotic cell death and other forms of DNA damage, and the distinction between different stages of the cell death process. In this thesis, we used several techniques to study cell death induced by perinatal asphyxia: 1) the TUNEL (terminal deoxynucleotidyl transferase (TdT)-mediated dUTP nick-end labeling) assay; 2) DNA markers (Hoechst 33342); 3) histological staining methods (Hematoxylin-Eosin and Nissl); 4) electron microscopy (EM) and 5) immunohistochemical staining was used to localize caspase-3 activated cells within different brain regions. In addition, we used a fluorometric assay designed to measure caspase-3 and caspase-3-like activity in tissue.

The TUNEL assay is based on the specific binding of TdT to a free 3'-OH end of DNA [73]. The 3' $\mathrm{OH}$ ends of DNA fragments are labelled either with biotin-dUTP, digoxigenindUTP, or FITC-dUTP mediated by TdT. This technique allows the detection of DNA strand breaks in individual cells in tissue sections and allows quantification of damaged cells by light microscopy. The TUNEL assay cannot be used to distinguish between apoptosis or necrosis, since positive cells may contain either laddered or randomly digested DNA and/or active DNA repair systems [40]. Major advantages of this technique are the ability to reveal early DNA breaks during apoptosis and quantification of the percentage of apoptotic cells by flow cytometric analysis or direct counting under a microscope. Furthermore, this technique can be combined with multiple immunofluorescence staining for cell markers to characterize cell types undergoing apoptosis or with in situ detection of mRNA expression [206].

Histological staining methods, such as Nissl and Hemotoxylin-Eosin (HE), visualize the morphological appearance of all cells and are therefore suitable for distinguishing normal cells from degenerating cells. Condensed chromatin and formation of apoptotic bodies are easily determined by examining tissue sections stained with Nissl or Hematoxylin [206]. With an electron microscope, morphological changes can be used such as swelling of organelles, membrane breakdown, cell disintegration (features of necrosis) or cytoplasmic density, chromatin condensation, membrane-bound apoptotic bodies (features of apoptosis). Features of necrosis and apoptosis as well as subtle changes in nuclear densities can be distinguished using electron microscopy [180]. A major disadvantage of the use of electron microscopy is the time-consuming analysis and complexity in both preparation of the tissue and analysis.

Immunohistochemical analysis of caspases or products of cleavage by caspases is possible now, since many antibodies were produced in the last years. Antibodies recognize the full length and/or large cleaved fragments of their respective proteins. For example, cleaved 


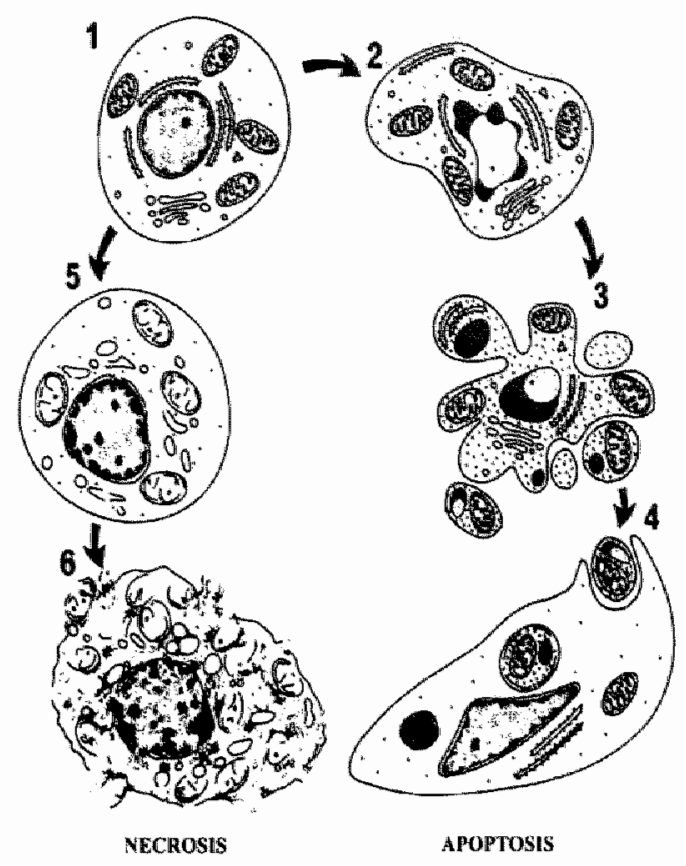

Figure 2.The morphological appearance of necrosis (left) and apoptosis (right). (1) a normal cell. At the onset of apoptosis in (2), there is compaction of chromatin around the outside of the nucleus and condensation of the cytoplasm. In (3) the cell surface has become convoluted, and apoptotic bodies are formed (nuclear fragments). The apoptotic bodies are ingested by neighbouring cells in (4). Necrosis begins in (5) with mitochondrial swelling and clumping of chromatin. This is followed in (6) by disruption of the membrane and loss of cell integrity. (From Kerr and Harmon, 1991; with permission).

caspase-3 antibody (Asp 175) detects endogenous levels of the large fragment (17/19 kDa) of activated caspase-3 resulting from cleavage adjacent to Asp 175 [64,172]. These antibodies can easily be combined with cell-specific markers for indentification of cell type.

Another method to detect apoptosis in tissue or cell cultures is fluorometric or colorimetric determination of artificial caspase substrates. These include acetyl-Asp-Glu-Val-Asp (DEVD) and acetyl-Tyr-Val-Ala-Asp (YVAD), linked either to 7-amino-4-mehtylcoumarin (AMC) dye or pNA. DEVD is cleaved by all of the caspases, except caspase-2, and can be used as a general measurement for caspase activity. YVAD is cleaved by caspase- 1 and caspase- 4 , poorly by caspase- 3 , and not cleaved by caspase-2, $-6,-7$ [219]. The selectivity of the substrates is based on the absolute caspase enzyme substrate cleavage site $\mathrm{C}$-terminal to aspartate. The cleavage rate for each substrate reflects the proteolytic activity of the caspases present in the tissue. A major advantage of this method is the fast quantitative analysis of caspase activity within the tissue. The selectivity of the different substrates is however still under debate. 


\section{Developmental apoprosis}

Apoptosis occurs throughout the CNS in neurons, glial and neural progenitor cells. It is estimated that at least half of the original cell population is eliminated as a result of apoptosis in the developing nervous system [27,160]. Apoptosis is essential for normal development, as it is important for optimisation of synaptic connections, removal of unnecessary neurons and pattern formation. Moreover, apoptosis acts as part of a control and repair mechanism that contributes to the high level of plasticity during development by compensating for many genetic or developmental errors. Incorrectly programmed cells fail to receive the trophic signal needed for their survival and consequently activate their suicide program.

The essential role of apoptosis during neural development is illustrated by knockout mice lacking caspase-3, caspase-8 or caspase-9 or Apaf- $1[39,92,130,131,252]$. These mice all have abnormal development: hyperplasia, disorganized cell distribution, craniofacial malformations and ventricular obstruction by mitotic and differentiating neurons [39,252]. Deletions of caspase -8 and caspase -9 are both lethal to the embryo, while caspase- 3 knockouts die in the first three weeks of life. Brain development is almost normal in caspase-1, and -11 knockout mice, suggesting that these caspase are less critical for normal neuronal development. These mice are deficient in interleukin-lalpha and - 1 beta and are resistant to lipopolysaccharide-induced endotoxic shock. In contrast, mice that over express the antiapoptotic gene bcl-2 or lack the pro-apoptotic gene bax exhibit increases in selected neuronal. subpopulations without gross malformations of the CNS. However, other researchers were able to show that cells during development can die in a bax and bcl-2 independent manner. These findings implicate a crucial role of apoptotic cell death during morphogenesis of the developing brain.

Levi-Montalcini and colleagues discovered the target-derived factor for sensory neurons as Nerve Growth Factor (NGF), for which they received the Nobel Prize [142,143]. NGF belongs to a family of neurotrophins that includes NGF, NT-3, BDNF and NT-4/5. These neurotrophins act on a family of tyrosine kinase receptors trkA, trkB, and trkC. Levi-Montalcini and coworkers showed that if neurotrophins or their receptors were absent, all neurons that require these factors would die. These discoveries led to the foundation of the neurotrophin hypothesis, which proposes that immature neurons compete for target-derived trophic factors, which are present in limited amounts [144]. Neurons that are successful in establishing correct synaptic connections would obtain trophic factors to support their survival. Glial cells seem to depend on trophic support during myelination or for the regulation of proliferation, differentiation and survival. For instance, several trophic factors, such as interleukin-1 (IL-1), Insulin-like Growth Factor (IGF-1), NT-3, were able to inhibit apoptosis in myelinating of differentiating oligendrocyte populations [10]. After the period of developmental cell death, neurons and glial cells are less sensitive to growth factor withdrawal.

Little is known about exact time course of pre- and postnatal developmental cell death in the different brain structures within the human or rat. There seem to be temporal differences in the timing of developmental apoptosis in various regions of the maturing brain. For example, in the pons and medulla of the rat physiological apoptosis occurs perinatally, whereas rat cerebellar neurons undergo physiological apoptosis during the first two postnatal weeks 
[220,247], thalamic nuclei of the rat exhibit apoptotic morphology in the first postnatal week [211], and cortical neurons of the rat die naturally during the first and second postnatal weeks $[65-67,99,162]$. The timing of these events is paralleled by an increase in the expression of bax and caspase- 3 [166].

During development, cell death is primarily a matter of cell suicide (apoptosis), which is carefully regulated by the caspases, bcl-2 family of genes and by survival signalling pathways. NGF withdrawal during development results in a decrease in mitogen-activated protein (MAP) kinase and phosphoiniostitide 3-kinase (PI3K) activity. The MAP kinase cascade includes subsequent phosphorylation and activation of the kinases Raf, MAP kinase/ERK kinase (MEK) and extracellular signal-regulated protein kinase (ERK). This cascade plays an important role in cell survival by activating the of pp90 ribosomal S6 kinase (RSK) protein family members. RSK can phosphorylate and inactivate BAD and activate the transcription factor CREB. CREB is known to activate transcription of bcl-2 and can stimulate cell survival directly. Active PI3K enzymes can then catalyse the formation of the lipid 3'-phosphorylated phophoinositides, which regulate the activity of Ser/Thr kinase Akt. Active Akt in turn supports the survival of neurons by activating key proteins including apoptosis regulators and transcription factors $[189,195]$.

NGF withdrawal not only leads to loss of survival factors, but also induces activation of proteins that can cause mitochondrial damage. After NGF removal, c-Jun amino $(\mathrm{N})$-terminal kinases (JNK, also known as stress-activated protein kinases) are activated leading to phosphorylation of c-Jun. This transcription factor in turn induces the expression of DP5. DP5 might translocate Bax from the cytosol to the mitochondria, causing mitochondrial damage. This results in the release of cytochrome $\mathrm{C}$, activation of caspase -9 and -3 , and eventually cell death (see for review [202]).

\section{Cell death as a consequence of perinatal asplyxia}

Hypoxia-ischemia induced neuronal death has long been considered to represent necrosis, but in the last decade several studies showed that many neurons undergo apoptosis after global or focal hypoxia-ischemia [174,231]. Furthermore, only recently researchers have started to accept the fact that the response to injury by the immature brain differs from the adult brain. Both mechanism and the rate of cell death appear to be different in the immature CNS after injury.

In the Rice-Vannucci model, perinatal asphyxia leads to necrosis in selective areas in the first few hours after the insult (primary damage). Thereafter a secondary response is triggered that can continue for days or weeks, resulting in a greater proportion of delayed brain damage, primarily apoptosis. This secondary response is often accompanied by an inflammatory reaction $[164,174]$. The temporal pattern of apoptosis varies in each brain region and is dependent on the age at which hypoxia-ischemia is induced. For example, in frontal and parietal cortex, striatum, globus pallidus and hippocampus, a relatively high density of apoptotic cells were observed from $6 \mathrm{hr}$ through 7 days after neonatal unilateral hypoxia-ischemia at P7 (Rice-Vanucci model) [169,174]. In the cingulate cortex, CA3, dentate 
gyrus and thalamus, a peak in density between 24 and 72 hr was found after the insult followed by a subsequent decline [175]. An increase in apoptotic density was not observed until 24 hrs after the insult in animals with mild to moderate brain damage [169].

In the global asphyxia model at term [15], apoptotic morphology was observed in the thalamus and anygdala, 1-4 days after birth. Increased number of fragmented nuclei was also observed in the hippocampus, striatum, frontal and piriform cortex and cerebellum during the 8 days after the insult, peaking at P8. There was no necrosis or inflammation observed in this model [54].

In the last decade, a plethora of studies have been published investigating the series of events leading to cell death after CNS injury in the adult brain. Little is known however, about the exact mechanisms involved in CNS injury after perinatal asphyxia. Whereas trophic factor withdrawal has a prominent role in physiological apoptosis, there is little evidence to implicate trophic factor withdrawal as a primary mechanism in perinatal asphyctic insults. Rather, glucose and oxygen deprivation may trigger mitochondrial impairment and excitotoxicity. It has been suggested that in pathological situations such as perinatal asphyxia, glutamate receptor stimulation is a direct consequence of energy failure [98,255]. Accordingly, it is hypothesized that this energy-linked excitoxicity may lead to hypopolarisation of neurons, NMDA-Rmediated influx of $\mathrm{Na}+$ and $\mathrm{Ca}^{2+}$ and mitochondrial failure.

It may be speculated that after hypoxic-ischemic injury in the develloping CNS different death pathways and regulatory mechanisms are activated within a single cell. For instance, free radicals such as superoxide anion $\left({ }^{\circ} 2^{\circ}\right)$, hydrogen peroxide $\left(\mathrm{H}_{2} \mathrm{O}_{2}\right)$, and the hydroxyl radical $(\cdot \mathrm{OH})$, can cause DNA strand breakage and base modification in DNA, lipid peroxidation with subsequent loss of membrane integrity and eventually to cytotoxicity [93]. Moreover, apoptosis due to DNA damage involves various cyclins and their specific cyclin-dependent kinases, which are important components of the cell cycle machinery. Upregulation of these cyclins by DNA damage leads to apoptosis [45]. The tumour suppressor protein, p53, is also involved in the apoptotic process after DNA damage. The exact mechanism by which p 53 promotes cell death is not known; several studies have suggested that p53 promotes Bax-mediated cell death. Furthermore, apoptosis after hypoxic-ischemic injury can be mediated by the activation of death receptors, such as Fas [173]. These receptors contain a death domain that is shared by an adapter molecule, named MORT-1/FADD. This molecule also contains a death effector domain which can bind to pro-caspase 8 , creating the death-inducing complex (DISC). When the DISC binds pro-caspase 8, autocleavage will occur. Active caspase-8 can act directly and indirectly (via mitochondria) to cleave caspase-3, leading eventually to apoptosis [202].

Neuronal necrosis may represent the morphological type of cell death resulting from a failure of cells to execute the full apoptotic program. Portera-Cailliau and co-workers showed that glutamate-induced excitotoxicity during the first week op development induces neuronal death with features of both apoptosis and necrosis (apoptosis-necrosis morphological continuum) [185]. This apoptosis-necrosis continum was also observed in rat pups after unilateral carotid artery occlusion at P7 (Rice-Vannucci model) [169,187]. However, prolonged caspase-3 activation [77] and the presence of cells with an apoptotic-like morphology up to days after 
the hypoxic-ischemic injury $[169,188]$ suggests that the apoptotic machinery plays an impotant role in cell death after hypoxia-ischemia in the immature brain.

In summary, several investigators have shown that the cascade of biochemical and molecular events after unilateral or bilateral carotid ligation (Rice-Vannucci model) includes Fas death receptor activation, mitochondrial distress, formation of free radicals, accumulation of cytochrome $c$ and cleavage of caspase 8 and caspase-3. Furthermore, alterations in the levels of bax and bcl-2 favouring apoptosis-promoting bax, were reported after neonatal hypoxiaischemia [173].

Little is known however about the signalling cascade leading to cell death after global asphyxia at term. Seidl et al. (2001) showed that perinatal asphyxia in this animal model caused energy depletion in the brain, heart and kidney [205]. Furthermore, it was shown that global asphyxia at term caused an increase in cerebral blood flow and brain tissue glucose accompanied with severe acidosis $[61,158]$. In addition, evidence for the involvement of glutamate toxicity and oxidative stress in the cell death mechanism following global asphyxia at term was provided by measuring the release of glutamate [124] and free radicals [36]. Glutamate release was still increased, 3 months after the asphyxial insult (birth). The release of free radicals was increased after 5 min of reoxygenation ( 5 min recovery). Expression of transcription factors and transporters and metabolic factors were found to be up- or downregulated in asphyctic rat pups $10 \mathrm{~min}$ after the global asphyctic insult, providing evidence for changes in gene response $[133,159]$. These data suggest that acidosis, energy depletion, overstimulation by excitatory amino acids (for example glutamate), and free radical-mediated reactions may be involved in the signalling transduction pathways towards cell death after global asphyxia at term.

\section{Nitric oxide as mediator of neuronal death}

Nitric oxide (NO) is an important signalling molecule that regulates several physiological processes including vasodilatation, inflammation, neurodevelopment and synaptic plasticity (see for reviews $[50,51,72]$ ). Furthermore, NO plays an important role in the synthesis of cyclic GMP, an important second messenger molecule in the CNS, by regulating guanylyl cyclase activity [23]. However, excessive production of NO can easily lead to neurotoxicity, because of its radical chemical properties. NO is a small lipid soluble molecule that can diffuse readily from the site of synthesis and act on neighbouring cells. The biological activity of $\mathrm{NO}$ is dependent on the rate of synthesis, concentration, rate of reaction with a target molecule and the concentration of a target molecule.

NO is produced by the enzymatic conversion of L-arginine, in L-citrulline and NO in the presence of oxygen and NADPH. This reaction is catalyzed by NO synthases (NOS). Three major isoforms of NOS have been identifed: neuronal NOS (nNOS) 22,24$]$, endothelial NOS (eNOS) [68,134], and inducible NOS (NOS) $[157,248]$. nNOS is mainly localized in neurons using the excitatory transmitter glutamate or inhibitory transmitter GABA. lit is also found in cholinergic and other aminergic cells in the striatum, cortex and hippocampus and in glutamergic cells of the cerebellum. Expression of iNOS is observed predominantly in 
microglia and astrocytes following injury. eNOS is localized in endothelial cells and a small population of neurons (hippocampal pyramidal and granule cells) within the CNS [52,234]. The catalytic activity of nNOS (and eNOS) is turned on by an increase in $\mathrm{Ca}^{2+}$ influx and calcium binding to calmodulin. The calmodulin binding site of nNOS triggers the electron transport cycle necessary for nNOS activity.

Several studies demonstrated that NO production could be involved in the regulation of apoptosis after hypoxic-ischemic damage. Under pathological conditions, excessive $\mathrm{NO}$ and $\mathrm{O}_{2}$ may react to form peroxynitrite (ONOO-), a highly reactive oxidant. Peroxynitrite can nitrate or hydroxylate protein tyrosine residues $[12,48,106]$, it oxidizes thiols $[190,213]$, or decomposes in $\mathrm{OH}$ and $\mathrm{NO} 2$, which both can initiate lipid peroxidation $[12,48]$. Possible mechanisms of neurotoxicity of peroxynitrite involve DNA damage and activation of poly(ADP-ribose) synthase (PARS) [216]. After activation, PARS promotes posttranslation protein modification by catalysing the transfer of $A D P$-ribose units onto acceptor proteins such as histones, and PARS itself. This leads to depletion of NAD and ATP and cell death. It has been suggested that $\mathrm{NO}$ also inhibits the activity of ribonuclease reductase and other DNA-repair enzymes, causing inhibition of cell proliferation [46]. Interaction of NO with iron-sulfur-containing enzymes involved in energy metabolism, such as cytochrome c oxidase, may lead to mitochondrial damage and loss of calcium homeostasis [21]. NO may also contribute to excitotoxicity by increasing the extracellular concentrations of various neurotransmitters, including glutamate, aspartate, through enhanced release and/or inhibition of reuptake [25].

Involvement of NO-mediated toxicity in the mechanism of cell death after perinatal asphyxia has been suggested in many studies. In the Rice-Vannucci model for perinatal asphyxia, nNOS immunoreactivity was increased up to P7 in brain regions ipsilateral to the injury, including cerebral cortex, caudate-putamen, and thalamus [107]. Suppression of this nNOS activity by injection of the selective nNOS inhibitor 7 -nitroindazole (7-NI) prior to the lesion reduced the brain damage induced by the hypoxic-ischemic insult considerably [108]. In addition, suppression of iNOS activity by injection of an inhibitor of INOS prior to and daily atter the lesion resulted in a decrease in NO production and focal lesion. In the global asphyxia model, short and long-term changes in cGMP levels and NOS activity were reported in the striatum and cortex $[63,151,152]$. However, there are no studies reporting a direct correlation between the NO production and the anount of cell death in this model. The available data show that NOS activity is increased after global asphyxia at term and that NO may play a role in the cell death process after perinatal asphyxial injury. 


\section{Brain structures and neurotransmitters}

\section{Basal ganglia}

The basal ganglia is a complex neuronal network that is involved in motor control, sensorimotor integration and learning [31]. It consists of four large subcortical nuclei: the striatum (caudate nucleus, putamen and nucleus accumbens), globus pallidus (GPi), the subthalamic nucleus (STN) and the substantia nigra (SN; pars compacta and pars reticulata). The striatum is the input structure of the basal ganglia, receiving afferents from the entire cerebral cortex, the thalamic nuclei and the midbrain serotonergic and dopaminergic neurons. The striatum projects to the pars reticulata of the $\mathrm{SN}$ and the GPi (output structures) through both a direct and an indirect pathway. The direct pathway consists of the GABA/substance $\mathrm{P} /$ dynorphincontaining striatonigral (SNR) and striatopallidal (GPi) projection neurons. The indirect striatal projection is provided by D2/enkephalin-containing neurons that project to the GPi. The GPi in turn provides an inhibitory projection to the SNR and to the subthalamic nucleus. The subthalamic nucleus provides an excitatory input to the SNR. These direct and indirect pathways have opposite effects on the GABAergic output neurons that project to the thalamic nuclei, the superior colliculus and the SNR (for review see [75]). The 'balance' between these two pathways appears to be essential for the normal regulation of movement [3,74]. Disturbance of the balance at the levels of the output centers of the basal ganglia are considered to be the cause of either hypokinetic disorders, such as Parkinson's disease (loss of dopamine), or hyperkinetic disorders, such as Huntington's disease (loss of subset of striatal neurons) [56]. Neurons in the striatum can be subdivided into two subtypes depending on frequence of spines on their dendrites: spiny and aspiny neurons [58]. The spiny neurons represent more than $90 \%$ of the total population of striatal neurons $[84,251]$. The remaining neurons are considered to be mainly interneurons. The spiny neurons all contain glutamic acid decarboxylase (GAD) the enzyme responsible for GABA production [122]. These striatal neurons receive excitatory (glutamatergic) input from the cortex, thalamus and amygdala (see for review [209]). Further, they receive inputs from dopamine afferents from the $\mathrm{SN}$, inhibitory GABA inputs from axon collaterals of other spiny neurons, inhibitory input from GABA striatal interneurons as well as inputs from cholinergic interneurons [75].

Both clinical and experimental studies have shown that the basal ganglia is especially vulnerable to a perinatal asphyctic insult and that lesions within the basal ganglia can lead to severe neurological deficits. For example, clinical case studies using computer aided tomography and conventional magnetic resonance imaging (MRI) have provided evidence of damage in basal ganglia and thalami as early as $6 \mathrm{~h}$ post-insult in several cases of perinatal asphyxia [8,47,2 10]. Moreover, infants with more severe lesions evident on brain MRI such as basal ganglia lesions, showed persistent and diffuse neurological abnormalities (spastic quadriplegia, choreoathetosis, and persistent feeding problems) after the first week of life [161,197]. Using the Rice-Vannucci model to induce neonatal hypoxia-ischemia (P7), Nakajima and co-workers showed that cell damage was profoundly present in both the thalamus and striatum [169,173]. In addition, increased striatal immunoreactivity of dynorphin A and 
decreased striatal immunoreactivity for substance $P$ were detected 7 days after the hypoxicischemic insult (at P14) whereas neuropeptide Y-like [114] and tyrosine hydroxylase immunoreactivity (enzyme necessary for synthesis of dopamine) were unaltered [29]. On the other hand, the striatal cholinergic system seems to be relatively resistant to the neonatal hypoxic-ischemic insult [28].

Similar observations were reported in studies using the global asphyxia model at term. However, global asphyxia seems to induce more long-lasting alterations in dopaminergic parameters within striatum, such as an increase in density of striatal TH [43] and DI and D2 dopamine receptor mRNA levels up to 4 weeks of age [86], and a decrease in striatal dopamine release at 6 months of age [154]. Striatal glutamate and aspartate levels were also decreased 6 months after the global asphyctic insult. In the substantia nigra, global asphyxia at term caused a decrease in both GABA and aspartate levels [154].

The above-clescribed studies provide evidence for both acute and chronic deficits in neurotransmission within the basal ganglia after perinatal asphyxia. In addition, these studies show that there is a selective vulnerability of different cell types within the basal ganglia to a perinatal asphyxial insult. The reason for this selective vulnerability is not known, but it is suggested that cell types with a high metabolic rate are more vulnerable for hypoxicischemic insult. Several studies have shown that excessive release of excitatory amino acids during asphyxia may contribute to the brain injury within the basal ganglia through overactivation of receptors $[14,140]$. This overstimulation might lead to a disruption of the neurotransmiitter systems leading to a chronic imbalance in the projection pathways that control movement. Tracer studies investigating the interactions between the neurotransmitter systems within the basal ganglia are needed to provide more detailed information regarding the disturbed projection pathways after perinatal asphyxia.

\section{Cerebellum}

The cerebellum plays an important role in sensorimotor coordination, adaptation, associative learning and acquisition of spatial memory [135]. Whereas cortical and hippocampal regions are involved in spatial and object recognition. The cerebellum seems to be involved in the navigation to reach a certain object, explore a new environment and acquire spatial information in relation to personal orientation [183]. The volume of the cerebellar cortex of pre-term term infants, compared to term infants, is strongly related to cognitive test performance, in particular the Wechsler Intelligence Scale for Children [4]. Furthermore, experimental studies with hemicerebellectomized (HCbed) rats demonstrated that the cerebellum is essential for development of efficient exploration strategies. These HCbed rats displayed circling behaviour in a maze and were not able to acquire the task if they did not learn the task before the surgery [184]. The cerebellar cortex consists of three layers: the granular cell layer, the Purkinje cell layer and the molecular cell layer. The granular cell layer borders on the central white matter of the cerebel/um and consists of small, glutamatergic neurons. The Purkinje cell layer contains large, GABAergic neurons (Purkinje cells), which are considered the output neurons of the cerebellum. These cells are typically located in a single row at the border 
of the granular and the molecular cell layer. The Bergmann glia cells (also called Golgi epithelial cells) lay between the larger Purkinje cells and provide feedback inhibition to the granular cells. The molecular cell layer has a low cell content and contains mainly dendritic branches of the Purkinje cells and the Bergmann glial fibers (for reviews see [237,238].

Purkinje cells are especially vulnerable to hypoxic-ischemic insults. The reason for the selective vulnerability of Purkinje cells is not fully understood, but several studies suggested that it may be related to the high expression of AMPA receptors by these neurons causing increased susceptibility to exctotoxic mediated cell death or to the high metabolic rate of these neurons [117]. Purkinje cells are rich in calcium and calcium-buffering systems, which are generally resistant to hypoxic-ischemic mediated cell death. Recently, Katsetos and co-workers showed that acute hypoxia in guinea pig fetus at term reduced calbindin-D28K immunoreactivity in Purkinje cells until $72 \mathrm{~h}$ after the insult [117]. More interesting, the same group of investigators showed that immunoreactivity of calbindin-D28K in cerebellar Purkinje cells from children with hypoxic-ischemic encephalopathy was decreased compared to children who died without neurological and neuropathological findings [136]. Loss of Ca" buffering proteins, such as calbindin-D28K, may contribute to delayed cell death or degeneration within the cerebellum after neonatal hypoxia-ischemia.

Although many behavioural studies have been performed using the Rice-Vannucci model of neonatal hypoxia-ischemia, none of these studies have investigated the relation between cerebellar damage and behavioural function. These studies are strictly related to structural changes within the hippocampus, basal ganglia or cortex. Using the global asphyxia model at term, Dell'Anna and co-workers demonstrated delayed neuronal death in the cerebellum at postnatal day 8 as a consequence of the asphyctic insult [54]. Furthermore, white matter damage within the cerebellum was detected 3 months after the global asphyctic insult at term [125]. Volumes of the different layers and total number of granule and Purkinje cells within the cerebellum were, however, not affected in asphyctic rats at 2 years of age [244]. These data provide further support for selective vulnerability of Purkinje cells to perinatal asphyxia. Loss of Purkinje cells or their synaptic contacts may be related to the behavioural deficits observed after perinatal asphyxia in both experimental settings and clinical cases. Further studies exploring the issue are necessary to investigate the direet relation between cerebellar structural changes and behavioural deficits after perinatal asphyxia.

\section{Behavioural outcome after perinatal asphyxia}

The extent of behavioural deficits produced by perinatal asphyxia can give an indication of the extent of the CNS damage and is important for evaluating the long-term consequences of post-asphyxial therapy. Locomotor behaviour is mostly studied using the open field task, which can provide measurements of anxiety or spontaneous activity $[102,153]$. Both young and adult rats display exploratory behaviour when subjected to a novel environment. It is believed that this behaviour is facilitated by arousal, curiosity and fear for an unfamiliar environment. 
Normally, rats start to explore new surroundings following a short delay after placing them in the middle of the open field. Fear and emotional state of the animal modulate this spontaneous activity.

To evaluate sensorimotor behaviour, a wide variety of tasks were developed, such as a the rotating treadmill, circling behaviour, Jatency to fall off a bridge or wire (grip task), the staircase task [165], foot-fault test [1 1], postural reflex test [18], limb-placing test [18] and foot print task $[132,224]$. These simple tests can easily be applied at any age and scoring is straight forward and unambiguous. Learning effects can however interfere with the interpretation of the degree of sensorimotor recovery, due to repeated trials. For example, the increase in time spent on the bridge over trials might be indicative of an underlying learning process or improved sensorimotor performance. This makes it difficult to interpret improvement over time within the same animal.

There are many behavioural tasks for measuring learning and memory-related behaviour. The Morris water escape task has proven to be an exceptionally useful tool to study learning and memory abilities of rats $[57,167]$. During this test, rats have to learn to locate a hidden platform with a fixed location in a water tank during repeated trials. The rat can use a learned sequence of movements leading it to the platform or it can use the cues/objects from the environment to orientate itself spatially (spatial reference memory). A probe trial, in which the platform has been removed, can be used to test whether the rats actually learned the position of the platform. During the probe trial, the spatial accuracy of the rat is determined, represented by the time spend in the quadrant where the platform used to be (target quadrant) or by the number of times it crosses the former platform location.

\section{Locomotor and sensorimotor deficits in animal models for perinatal asphyxia}

Animal studies using the Rice-Vannucci model (unilateral carotid ligation with subsequent hypoxia at P7) demonstrated motor deficits in the rotarod test [11 10,111,239], grip task [18] and asymmetry in limb placing and foot-faults [18] at 6 weeks of age. In addition, motor function was abnormal in the postural reflex test at this age [18] and a marked impairment in the staircase task at 3 months of age [227]. In the open field task after apomorphine administration, lesioned rats showed a marked turning behaviour ipsilaterial to the llesioned side $[6,239]$. Spontaneous activity in the open field task was increased at weaning but not at 3 months of age [6].

Most experimental studies investigating motor deficits after term global asphyxia describe a decrease in locomotor activity ( 5 months of age) and anxiety ( 3 months of age) in the open field task for male rats $[102,153]$. Hoeger and co-workers, however, did not observe this hypoactivity in the open field task at 3 months of age [102]. At 4 weeks of age, Chen and coworkers (1995) observed an increase in spontaneous activity, motility and rearing ( $-25 \%)$ after $15-16$ min of asphyxia, while following $19-20$ min of asphyxia these parameters were decreased $(-50 \%)$, as compared to control rats. There were no differences observed in sensorimotor behaviour between asphyctic and control rats using measures of limb clasping, limb placing or turning on an inclined grid [17] at 6 weeks of age, or of the grip task or rotarod 
task at 24 months of age [244].

The above-described studies show that long-lasting locomotor deficits are induced by both hypoxia-ischemia (Rice-Vannucci model) and global asphyxia at term. The locomotor deficits were more subtle and less consistent in the global asphyxia model. In addition, sensorimotor deficits were observed after hypoxia-ischemia at P7 (Rice-Vannucci) but not after global asphyxia at term.

\section{Learning $\&$ Memory performance after perinatal asphyxia in the rat}

In the Rice-Vannucci model, perinatal asphyxia resulted in learning impairments in choice reaction time task, plus-maze tasks, T-maze acquisition and Morris water escape task (long-term reference memory), tested between 6 and 16 weeks of age $[6,7,105,239,253]$. Impairment of short-term memory tested using the radial maze tasks was transient in this model, tested between 7 and 12 weeks of age [105]. Furthermore, lesioned rats were not deficient in performing a passive avoidance paradigm at 10 weeks of age.

Global asphyxia at term had no effect on acquisition of spatial learning in the Morris water maze at 6 weeks of age $[17,153]$. At 4 months of age, asphyctic rats $(10,15$ or $20 \mathrm{~min}$ of asphyxia) were impaired in the initial acquisition of a spatial learning task in the Morris water maze as compared to control rats [17], indicating procedureal learning deficits. At 24 months of age, asphyctic rats were impaired in the ability to relearn the location of the platform after changing the position of the platform [244]. There were no deficits found in asphyctic rats in the multiple T-maze at 6 weeks of age [17] or 3 months of age [102].

\section{Regeneration following perinatal asphyxia}

Animal and human studies have shown that the injured CNS is capable of reorganisation. For example, the recovery of function in patients with unilateral brain damage in the perinatal period was demonstrated in an fMRI study. Finger movements of the affected hand produced widespread activation of the intact, ipsilateral hemisphere, suggesting that new ipsilateral projections were developed and functional [35]. Several criteria need to be met before functional recovery is possible. First, the injured neuron must survive and damaged axons must be able to regrow towards their original neuronal targets. Furthermore, the axons need to be renyelinated and functional synapses have to be formed on the surface of the target neuron before reconnection can be established [103]. The extent of functional recovery (regeneration capacity) after injury seems to depend on the age of the patient, location and extent of the lesion as well as individual variations in anatomical and functional connections (see for review [42]). Moreover, environmental factors, such as living in an enriched environment and physical rehabilitation, can lead to a robust increase in regeneration capacity [232].

Perinatal asphyxia occurs at a time when the brain is undergoing enormous growth and remodelling. Almost $2 / 3$ of the total number of cells in the brain is produced after birth. 
Stem and progenitor cells within the brain can proliferate and differentiate into new neurons or glial cells. These precursor cells occur in many regions of the brain, including the subventricular zone (SVZ), subgranular zone of the dentate gyrus ( $S G Z$ ) and the granular cell layer of the cerebellum $[70,116,233]$. The SVZ is the major source of myelinating oligodendrocytes and astrocytes within the forebrain during prenatal development $[146,147]$. In the neonatal and juvenile rats, SVZ cells do not migrate rostrally to the olfactory bulb as SVZ newly generated cells do in the adult rats [146]. The age at which SVZ cells switch from glial production to olfactory bulb neurogenesis has not been established yet. New neurons produced within the SGZ in the adult animal migrate into the adjacent dentate gyrus granule cell layer $[81,155]$. Proliferation and differentiation within the SVZ and SGZ is regulated by many factors including glucocorticoids [33], sex hormones [222], growth factors $[120,129,241]$, excitatory neurotransinission [32,34], learning [80], and stress $[139,221]$ (see for an overview of enhancers and inhibitors of neurogenesis [182]).

Recently, Levison and co-workers reported that hypoxia-ischemia at P7 (Rice-Vannucci model) produces long-lasting depletion of the SVZ progenitor cells. Futhermore, they showed that many of the dying cells after perinatal asphyxia within the SVZ were oligodendrocyte progenitor cells. At 3 weeks of age, the subcortical white matter of the rats, which had suffered hypoxia-ischemia at P7, was poorly myelinated, depleted of oligodendrocytes and enriched in astrocytes [148]. These data suggest that perinatal hypoxia-ischemia can diminish the total number of cells within the brain by disruption of proliferation.

In adult rats, proliferation has been shown to be upregulated after injury in the SVZ and SGZ and cortex $[112,113,217,249]$. Furthermore, newly made cells within the SGZ were able to migrate into the granule cell layer of the hippocampus and differentiate into neuronal cells within 2 months after global ischemia in the adult rat [109]. Moreover, one study provided evidence for newly generated neurons within adult humans. For example, Eriksson et al. described a study with terminally ill patients, which had received the proliferation marker bromodeoxyuridine (BrdU) to monitor tumour cell proliferation. Samples of the hippocampus and $S V Z$ of these patients were analysed post-mortem and newborn neurons were detected in the granule cell layer of all individuals [62]. These data show that neuronal regeneration can occur after injury within the adult brain. Furthermore, since neural precursors are present throughout life, stimulation of endogenous precursors could have therapeutic potential. Growth factors, such as BDNF, NGF and insulin-like growth factor-1 (IGF-1), play an important role in regulating neurogenesis and synaptogenesis. For example, administration of BDNF into the lateral ventricle has been shown to result in enhanced neurogenesis in the olfactory bulb and striatum $[13,181]$. Furthermore, administration of NGF to lesioned rats (unilateral decortication) increased the number of cholinergic boutons and synaptic contacts within the cortex [71]. Overexpression of IGF-I was also shown to produce a persistent increase in total number of neurons (neurogenesis) and synapses (synaptogenesis) in the hippocampus during postnatal development [177]. These growth factors are strongly expressed during recovery of hypoxic-ischemic insults in rats $[91,94,156]$. Treatment studies have proven that these factors are able to offer neuroprotection after injury in the neonatal rat when administered centrally or systemically $[5,88,1.21,208]$. 
Little is known about regenerative processes within the CNS after perinatal asphyxia of neonatal hypoxia-ischemia. Since the neonatal brain is more receptive to trophic factors, it is suggested that synaptic rearrangement, neurochemical compensation and regenerative sprouting can compensate for the loss of neurons in the developing CNS. The challenge in the absence of a direct treatment for perinatal asphyxia is to learn to enhance adaptive compensatory responses and modify those that do not promote normal function including for example glial scar formation.

\section{Therapeutic strategies}

As discussed previously, perinatal asphyxia leads to brain damage which starts during the hypoxic-ischemic insult itself (primary damage) and can continue for days or weeks (secondary damage). The aim of therapeutic intervention in perinatal asphyxia is to prevent the delayed neuronal death (secondary damage) and to promote functional recovery after brain injury.

Knowledge about the molecular mechanisms involved in the pathophysiology of perinatal asphyxia has led to the following therapeutic strategies: 1) prevention of energy depletion, for example by administration of barbiturates or mild hypothermia;2) decrease in excitotoxicity, for example by blocking NMDA receptors or calcium uptake, or by free radical scavengers; 3 ) reducing inflammatory response, for example by employing antiinflammatory agents; 4) blockade of intracellular events, for example by NOS inhibitors/ scavengers, free radical scavengers or anti-apoptotic agents; 5) stimulation endogenous protection mechanisms, for example by employing growth factors; 6) combined treatment, for example employing a cocktail containing protease inhibitors and receptor antagonists (see for reviews $[138,176,178,236]$ ).

These therapeutic strategies have predominantly or exclusively been tested in animal models for hypoxic-ischemic damage. Their application to the newborn human term infant has proven to be a great challenge. Many of these promising therapeutic strategies failed to produce clinically significant improvements in adults or are toxic. For example, clinical studies investigating therapeutic strategies in humans with acute stroke failed to show any benefit for the patient's recovery in the use of calcium channel blockers or free radical scavengers. Moreover, studies investigating the use of NMDA antagonists or the anti-inflammatory agents in the pathophysiology of stroke were terminated because of toxic side effects $[9,20,53,87,257]$.

In the term human infant, barbiturates were used for the treatment of seizures in neonatal hypoxic-ischemic encephalopathy. These studies did not however produce consistent results. For example, a randomized controlled trial of 32 asphyctic term infants that were given thiopental by constant infusion over 24 hrs, showed that this treatment did not reduce the number of seizures or mortality rate. Neurological outcome between the asphyxiated and control group was also similar [78]. An earlier study suggested benefits due to of the use 
of pentobarbital administered shortly after birth [215]: In addition, calcium channel blockers and free radical scavengers were used in the human asphyxiated term infants to reduce brain damage. A clinical study in asphyxiated infants using nicardipine, a calcium channel blocker, as a treatment had to be discontinued because of adverse hemodynamic effects of this agent [141]. The effect of allopurinol ( $40 \mathrm{mg} / \mathrm{kg}$ intraveously), a xanthine-oxidase inhibitor and free radical scavenger, was tested on the free radical status and outcome of severely asphyxiated infants. Allopurinol was able to decrease the amount of free radicals in plasma, maintain stable cerebral blood volume and electrical brain activity without inducing toxic side effects [230]. These case studies provide evidence for the use of drugs after perinatal asphyxia but deserve a critical approach. Potent drugs, such as allopurinol, have to be tested in randomized multicentre trials before clinical application is possible.

Although many drugs failed to provide neuroprotection in the human term infant, they were able to reduce damage in experimental animals. This discrepancy is probably due to the short time window of damage in the animal studies compared to humans. More important, all these therapeutic strategies block just one individual component of the signal pathways towards cell death leaving the door open for other inducers of cell death.

One of the most promising therapies for brain damage as a consequence of perinatal asphyxia is mild hypothermia. Several studies have shown that post-asphyxia mild hypothermia is safe and easy to apply $[89,90]$. There is, however, currently no study published describing a beneficial effect of hypothermia in the term infant. Numerous animal studies showed that mild hypothermia can have a neuroprotective effect by affecting multiple downstream mechanisms of cell injury. For example, post-insult hypothermia $\left(30^{\circ} \mathrm{C}\right)$ for 24 or $26 \mathrm{~h}$, starting directly or $2 \mathrm{~h}$ after the neonatal hypoxic-ischemic insult (Rice-Vannucci model at P7) reduced the size of cerebral infarction [239] and caspase- 3 activity at 6 and $24 \mathrm{~h}$ after the insult $[69,226]$. Post-insult hypothermia $\left(30^{\circ} \mathrm{C}\right)$ for $26 \mathrm{~h}$ was able to improve cognitive performance in the Morris water maze at 6 weeks of age. This hypothermia paradigm did not affect functional outcome in control pups [239]. A larger decrease in temperature $\left(21^{\circ} \mathrm{C}\right)$ did not improve cognitive performance after hypoxia-ischemia, but prevented motor deficits [253]. However, a number of studies using the Rice-Vannucci model showed that the brain damage was not prevented but simply delayed by mild hypothermia $\left(32^{\circ} \mathrm{C}, 3 \mathrm{~h}\right)[229]$. The outcome probably depends on the temperature and time-interval of hypothermia. Recent studies showed that mild hypothermia $\left(30^{\circ} \mathrm{C}\right)$ is most effective in reducing cerebral injury in the Rice-Vannucci model when applied in the first $72 \mathrm{~h}$ after the hypoxic-ischemic insult $[207,223]$.

The effect of hypothermia on the outcome of perinatal asphyxia was also studied using the global asphyxia model at term [15]. Hypothermia, during $20 \mathrm{~min}$ at $15^{\circ} \mathrm{C}$ and after $5 \mathrm{~min}$ at $15^{\circ} \mathrm{C}$ global asphyxia, prevented an increase in NOS-dependent cGMP synthesis (measured at $\mathrm{P} 10$ ) and a decrease in NOS-immunoreactive neurons (measured at 6 months of age) [37,152]. Furthermore, hypothermia during the asphyxial insult was also able to prevent hypoactivity in the open field task at 5 months of age [153]. 
The above described studies illustrate the potential benefit of post-asphyxial hypothermia treatment. Although the exact mechanism of protection by hypothermia is unknown, these studies show that hypothermia can reduce cerebral damage probably by a reduction of energy depletion, reduction of free radical formation, nitric oxide synthesis and caspase activation. Furthermore, it is postulated that hypothermia can lower excitatory amino acid secretion, downregulate glutamate receptors $[30,150,171,258]$ and reduce inflammatory response $[41,228]$.

Unfortunately, there are only few studies investigating long-term outcome after post-asphyxia hypothermia. It has been suggested that hypothermia might be able to prevent cerebral damage transiently but not permanently. Therefore, more studies are necessary to study this important issue at the morphological, biochemical and behaviour level. However, even if hypothermia is not able to protect permanently, above mentioned studies show that hypothermia can provide a therapeutic window. As such it seems particularly interesting to combine hypothermia with caspase inhibitors and anti-inflammatory agents. Recently, Adachi and coworkers (2001) showed that a combination of hypothermia $\left(29^{\circ} \mathrm{C}\right.$ for $\left.\mathbb{l} \mathrm{h}\right)$ and a caspase inhibitor, boc-aspartyl-(OMe)-fluoromethyl-ketone (BAF), provided a strong protective effect against caspase activity, measurement $16 \mathrm{~h}$ after the insult, and neuronal damage at 7 days of age [1]. This study encourages further investigations using combined hypothermia and drug treatment.

\section{Conclusion}

Short and long-term consequences of perinatal asphyxia cam easily be studied using animal models inducing either hypoxia-ischemia or global asphyxia. Hypoxia-ischemia (RiceVannucci model) produces mild or severe focal lesions depending on the duration of hypoxia and age of induction. Global asphyxia does not produce an infarct, but damages brain areas in which blood flow is reduced. Both necrotic and apoptotic features were observed in degenerating cells after hypoxia-ischemia, whereas only apoptotic features were visible in brain areas affected by global asphyxia at term. Furthermore, hypoxia-ischemia induces an inflammatory response, in contrary to global asphyxia at term. Mitochondrial failure and oxidative stress seem to be responsible for the activation of caspases and eventual cell death after hypoxia-ischemia or global asphyxia.

The basal ganglia, hippocampus and cerebellum are particularly vulnerable to an asphyxial insult. Loss of neurons in these regions can result in the functional deficits observed after perinatal asphyxia, such as locomotor, sensorimotor and learning deficits. Long-lasting locomotor and learning deficits were observed after hypoxia-ischemia and global asphyxia, whereas sensorimotor deficits were only observed after hypoxia-ischemia.

We conclude that both models are able to resemble clinical features of perinatal asphyxia. Hypoxia-ischemia induced at P7, however, induces more severe grey matter damage, whereas global asphyxia ( $\mathrm{P} 0$ ) produced mild grey and white matter damage. This is probably the result of the different time points of inductions ( $\mathrm{P} 7$ versus $\mathrm{P} 0$ ), since young rat are more resistant 
to hypoxia-ischemia than older rats. Furthermore, there seems to be a direct relation between the extent of structural damage within specific brain regions and the functional deficits after the insult in both models.

Long-term functional consequences are dependent on the extent of the injury and regenerative capacity. Understanding the mechanisms of neuronal plasticity is essential in order necessary to design appropriate strategies to up- or down-regulate plasticity changes and to promote recovery of function after perinatal asphyxia. Little is known however about the regeneration capacity and compensatory mechanisms after neonatal hypoxia-ischema or global asphyxia. Although there is no intervention that can be recommended for the treatment of clinical brain clamage after perinatal asphyxia on the basis of consistent randomized trial evidence, considerable progress has been made concerning the use of post-insult mild hypothermia. Post-asphyxia hypothermia has been proven to be effective in animal models and non-toxic in infants. A large multicentre trial of mild cooling for infants that suffered of perinatal asphyxia is currently in progress. 


\section{References}

[1] M. Adachi, O. Sohma, S. Tsuneishi, S. Takada and H. Nakamura. 2001. Combination effect of systemic hypothermia and caspase inhibitor administration against hypoxic-ischemic brain damage in neonatal rats. Pediatr Res 50: 590-5.

[2] J.M. Adams and S. Cory. 1998. The Bcl-2 protein family: arbiters of cell survival. Science 281: $1322-6$.

[3] G.E. Alexander and M.D. Crutcher. 1990. Functional architecture of basal ganglia circuits: neural substrates of parallel processing. Trends Neurosci 13: 266-71.

[4] M. Allin, H. Matsumoto, A.M. Santhouse, C. Nosarti, M.H. AlAsady, A.L. Stewart, L. Rifkin and R.M. Murray. 2001. Cognitive and motor function and the size of the cerebellum in adolescents born very pre-term. Brain 124:60-6.

[5] C.R. Almli, T.J. Levy, B.H. Han, A.R. Shah, J.M. Gidday and D.M. Holtzman. 2000. BDNF protects against spatial memory deficits following neonatal hypoxia-ischemia. Exp Neural 166: 99-114.

[6] W.Balduini, V. De Angelis, E. Mazzoni and M. Cimino. 2000. Long-lasting behavioral alterations following a hypoxic/ischemic brain injury in neonatal rats. Brain Res 859: 318-25.

[7] W. Balduini, V. De Angelis, E. Mazzoni and M. Cimino. 2001. Simvastatin protects against long-lasting behavioral and morphological consequences of neonatal hypoxic/ischemic brain injury. Stroke 32: 2185-91.

[8] A.J. Barkovich, K. Baranski, D. Vigneron, J.C. Partridge, D.K. Hallam, B.L. Hajnal and D.M. Ferriero. 1999. Proton MR spectroscopy for the evaluation of brain injury in asphyxiated, term neonates. AJNR Am J Neuroradiol 20: 1399-405.

[9] F.C.Barone and G.Z. Feuerstein. 1999. Inflammatory mediators and stroke: new opportunities for novel therapeutics. J Cereb Blood Flow Metab 19: 819-34.

[10] B.A. Barres, I.K. Hart, H.S. Coles, J.F. Burne, U.T. Voyvodic W.D. Fichardson and M.C. Raff. 1992. Cell death in the oligodendrocyte lineage. J Neurobiol 23: 1221-30.

[11] T.M. Barth and B.B. Stanfield. 1990. The recovery of forelimb-placing behavior in rats with neonatal unilateral cortical damage involves the remaining hemisphere. J Neurosci 10: 344959.

[12] J.S. Beckman, J. Chen, J.P. Crow and Y.Z. Ye. 1994. Reactions of nitric oxide, superoxide and peraxynitrite with superoxide dismutase in neurodegeneration. Prog Brain Res 103: 371-80.

[13] A. Benraiss, E. Chmielnicki, K. Lerner, D. Roh and S.A. Goldman. 2001. Adenoviral brainderived neurotrophic factor induces both neostriatal and olfactory neuronal recruitment from endogenous progenitor cells in the adult forebrain. J Neurosci 21: 6718-31.

[14] R. Berger and Y. Garnier. 1999. Pathophysiology of perinatal brain damage. Brain Res Brain Res Rev 30: 107-34.

[15] B. Bjelke, K. Andersson, S.O. Ogren and P. Bolme. 1991. Asphyctic lesion: proliferation of tyrosine hydroxylase-immunoreactive nerve cell bodies in the rat substantia nigra and functional changes in dopamine neurotransmission. Brain Res 543: 1-9.

[16] R.M. Blumberg, D.L. Taylor, X. Yue, K. Aguan, J. McKenzie, E.B. Cady, C.P. Weiner, H. Mehmet and A.D. Edwards. 1999. Increased nitric oxide synthesis is not involved in delayed cerebral energy failure following focal hypoxic-ischemic injury to the developing brain. Pediatr Res 46: 224-31.

[17] P. Boksa, A. Krishnamurthy and W. Brooks. 1995. Effects of a period of asphyxia during birth on spatial learning in the rat. Pediatr Res 37: 489-96.

[18] E. Bona, B. B. Johansson and H. Hagberg. 1997. Sensorimotor function and neuropathology five to six weeks after hypoxia-ischemia in seven-day-old rats. Pediatr Res 42: 678-83. 
[19] F.T. Bosman, B.C. Visser and J. van Oeveren. 1996. Apoptosis: pathophysiology of programmed cell death. Pathol Res Pract 192: 676-83.

[20] M.P. Bowes, F. Rothlein, S.C. Fagan and J.A. Zivin. 1995. Monoclonal antibodies preventing leukocyte activation reduce experimental neurologic injury and enhance efficacy of thrombolytic therapy. Neurology $45: 815-9$.

[21] C.S. Boyd and E. Cadenas, 2002. Nitric oxide and cell signaling pathways in mitochondrialdependent apoptosis. Biol Chem 383: 411-23.

[22] D.S. Bredt; P.M. Hwang, C.E. Glatt, C. Lowenstein, R.R. Reed and S.H. Snyder. 1991. Cloned and expressed nitric oxide synthase structurally resembles cytochrome P-450 reductase. Nature 351:714-8.

[23] D.S. Bredt and S.H. Snyder. 1989. Nitric oxide mediates glutamate-linked enhancement of cGMP levels in the cerebellum. Proc Natl Acad Sci U S. A 86: 9030-3.

[24] D.S. Bredt and S.H. Snyder. 1990. Isolation of nitric oxide synthetase, a calmodulin-requiring enzyme. Proc Natl Acad Sci U S A 87: 682-5.

[25] G.C. Brown and V. Borutaite. 2001. Nitric oxide, mitochondria, and cell death. IUBMB Life 52: $189-95$.

[26] B. Buendia, A. Santa-Maria and J.C. Courvalin. 1999. Caspase-dependent proteolysis of integral and peripheral proteins of nuclear membranes and nuclear pore complex proteins during apoptosis. J Cell Sci 112 ( Pt 11): 1743-53.

[27] M.J. Burek and R.W. Oppenheim. 1996. Programmed cell death in the developing nervous system. Brain Pathol 6:427-46.

[28] R.E. Burke and N. Kenyon. 1991. The effect of neonatal hypoxia-ischemia on striatal cholinergic neuropil: a quantitative morphologic analysis. Exp Neurol 113: 63-73.

[29] R.E. Burke and A. Reches. 1991. Preserved striatal tyrosine hydroxylase activity, assessed in vivo, following neonatal hypoxia-ischemia. Brain Res Dev Brain Res 61: $277-80$.

[30] R. Busto, M.Y. Globus, W.D. Dietrich, E. Martinez, I. Valdes and M.D. Ginsberg. 1989. Effect of mild hypothermia on ischemia-induced release of neurotransmitters and free fatty acids in rat brain. Stroke 20: 904-10.

[31] P. Calabresi, M. De Murtas and G. Bernardi. 1997. The neostriatum beyond the motor function: experimental and clinical evidence. Neuroscience 78: 39-60.

[32] H.A. Cameron, B.S. McEwen and E. Gould. 1995. Regulation of adult neurogenesis by excitatory input and NMDA receptor activation in the dentate gyrus. J Neurosci 15:4687 92.

[33] H.A. Cameron and R.D. McKay. 1999. Restoring production of hippocampal neurons in old age. Nat Neurosci 2: 894-7.

[34] H.A. Cameron, P.Tanapat and E. Gould. 1998. Adrenal steroids and N-methyl-D-aspartate receptor activation regulate neurogenesis in the dentate gyrus of adult rats through a common pathway. Neuroscience 82: 349-54.

[35] Y. Cao, E.M. Vikingstad, P.R. Huttenlocher, V.L. Towle and D.N. Levin. 1994. Functional magnetic resonance studies of the reorganization of the human hand sensorimotor area after unilateral brain injury in the perinatal period. Proc Natl Acad Sci U S A 91: 9612-6.

[36] F. Capani, C.F. Loidl, F. Aguirre, L. Piehl, G. Facorro, A. Hager, T. De Paoli, H. Farach and J. Pecci-Saavedra. 2001. Changes in reactive oxygen species (ROS) production in rat brain during global perinatal asphyxia: an ESR study. Brain Res 914: 204-7.

[37] F. Capani, F. Loidl, J.J. Lopez-Costa, A. Selvin-Testa and J.P. Saavedra, 1997. Ultrastructural changes in nitric oxide synthase immunoreactivity in the brain of rats subjected to perinatal asphyxia: neuroprotective effects of cold treatment. Brain Res 775: 11-23.

[38] B.S. Carter, A.D. Haverkamp and G.B. Merenstein. 1993. The definition of acute perinatal asphyxia. Clin Perinatol 20: 287-304. 
F. Cecconi, G. Alvarez-Bolado, B.I. Meyer, K.A. Roth and P. Gruss, 1998. Apaty (CED-4 homolog) regulates programmed cell death in mammalian development. Cell 94: 727-37.

[40] C. Charriaut-Marlangue and $Y$. Ben-Ari. 1995. A cautionary note on the use of the TUNEL stain to determine apoptosis. Neuroreport 7: $61-4$.

[41] K. Chatzipanteli, O.F. Alonso, S. Kraydieh and W.D. Dietrich. 2000. Importance of posttraumatic hypothermia and hyperthermia on the inflammatory response after fluid percussion brain injury: biochemical and immunocytochemical studies. $J$ Cereb Blood Flow Metab 20: 531-42.

[42] R. Chen, L.G. Cohen and M. Hallett. 2002. Nerwous system reorganization following injury. Neuroscience 111: 761-73.

[43] Y. Chen, M. Herrera-Marschitz, B. Bjelke, M. Blum, J. Gross and K. Andersson, 1997. Perinatal asphyxia-induced changes in rat brain tyrosine hydroxylase- immunoreactive cell body number: effects of nicotine treatment. Neurosci Lett 221: 77-80.

[44] Y. Chen, M. Hillefors-Berglund, M. Herrera-Marschitz, B. Bjelke, J. Gross, K. Andersson and $G$. von Euler. 1997. Perinatal asphyxia induces long-term changes in dopamine D1, $D 2$, and D3 receptor binding in the rat brain. Exp Neurol 146: 74-80.

[45] A. Copani, D. Uberti, M.A. Sortino, V. Bruno, F. Nicoletti and M. Memo. 2001. Activation of cell-cycle-associated protelins in neuronal death: a mandatory or dispensable path? Trends Neurosci 24: 25-31.

[46] R.S. Costa and $\downarrow$. Assreuy. 2002. Nitric oxidle inhibits irreversibly P8:15 cell proliferation: involvement of potassium channels. Cell Prolif $35: 321-32$.

[47] F.M. Cowan, J.M. Pennock, J.D. Hanrahan, K.P. Manji and A.D. Edwards. 1994. Early detection of cerebral infarction and hypoxic ischemic encephalopathy in neonates using diffusion-weighted magnetic resonance imaging. Neuropediatrics 25: 172-5.

[48] J.P. Crow and J.S. Beckman. 1995. Reactions between nitric oxide, superoxide, and peroxynitrite: footprints of peroxynitrite in vivo. Adv Pharmacol 34: 17-43.

[49] M. Dambska, L. Dydyk, T. Szretter, J. Wozniewicz and R.E. Myers. 1976. Topography of lesions in newborn and infant brains following cardiac arrest and resuscitation. Damage to brain and hemispheres. Biol Neonate 29: 194-206.

[50] T.M. Dawson, Gonzalez-Zulueta, M., Kusel, J., Dawson, V.L. 1998. Nitric oxide: diverse actions in the central and peripheral nervous systems. The Neuroscientist 4: 96-1 112.

[51] V.L. Dawson and T.M. Dawson. 1998. Nitric oxide in neurodegeneration. Prog Brain Res 118: $215-29$.

[52] J. De Vente, D.A. Hopkins, M. Markerink-Van Ittersum, P.C. Emson, H.H. Schmidi and H.W. Steinbusch. 1998. Distribution of nitric oxide synthase and nitric oxide-receptive, cyclic GMP-producing structures in the rat brain. Neuroscience 87:207-41.

[53] T.J. DeGraba. 1998. The role of inflammation after acute stroke: utility of pursuing antiadhesion molecule therapy. Neurology 51: S62-8.

[54] E. Dell'Anna, Y. Chen, E. Engildawork, K. Andersson, G. Lubec, J. Luthman and M. HerreraMarschitz. 1997. Delayed neuronal death following pierinatal asphyxia in rat. Exp Brain Res 115: $105-15$.

[55] E. Dell'Anna, M.C. Geloso, M. Magarelli and M. Molinari. 1996. Development of GABA and calcium binding proteins immunoreactivity in the rat hippocampus following neonatal anoxia. Neurosci Lett 211: 93-6.

[56] M.R. DeLong. 1990. Primate models of movement disorders of basall ganglia origin. Trends Neurosci 13: $281-5$.

[57] R. D'Hooge and P.P. De Deyn. 2001. Applications of the Morris water maze in the study of learning and memory. Brain Res Brain Res Rev 36: 60-90.

[58] M. Difiglia, P. Pasik and T. Pasik. 1976. A Golgi study of neuronal types in the neostriatum of 
monkeys. Brain Res 114: 245-56.

[59] J. Dobbing. 1971. Undernutrition and the developing brain: the use of animal models of elucidate the human problem. Psychiatr Neurol Neurochir 74:433-42.

[60] J. Dobbing and J. Sands. 1979. Comparative aspects of the brain growth spurt. Early Hum Dev 3: 79-83.

[61] E. Engidawork, Y. Chen, E. Dell'Anna, M. Goiny, G. Lubec, U. Ungerstedt, K. Andersson and $M$. Herrera-Marschitz. 1997. Effect of perinatal asphyxia on systemic and intracerebral $\mathrm{pH}$ and glycolysis metabolism in the rat. Exp Neurol 145: 390-6.

[62] P.S. Eriksson, E. Perfilieva, T. Bjork-Eriksson, A.M. Albom, C. Nordborg, D.A. Peterson and F.H. Gage. 1998. Neurogenesis in the adult human hippocampus. Nat Med 4:1313-7.

[63] C. Fabian Loid!, F. Capani, J.J. Lopez-Costa, A. Selvin-Testa, E.M. Lopez and J. PecciSaavedra. 1997. Long term changes in NADPH-diaphorase reactivity in striatal and cortical neurons following experimental perinatal asphyxia: neuroprotective effects of hypothermia. Int J Neurasci 89: 1-14.

[64] T. Fernandes-Alnemri, G. Litwack and E.S. Alnemri. 1994. CPP32, a novel human apoptotic protein with homology to Caenorhabditis elegans cell death protein Ced-3 and mammalian interleukin-1 beta-converting enzyme. J Biol Chem 269: 30761-4.

[65] I. Ferrer, E. Bernet. E. Soriano, T. del Rio and M. Fonseca. 1990. Naturally occurring cell death in the cerebral cortex of the rat and removal of dead cells by transitory phagocytes. Neuroscience $39: 451-8$.

[66] J. Ferrer, T. Serrano and E. Soriano. 1990. Naturally occurring cell death in the subicular complex and hippocampus in the rat during development. Neurosci Res 8: 60-6.

[67] B.L. Finlay and M. Slattery. 1983. Local differences in the amount of early cell death in neocortex predict adult local specializations. Science 219: 1349-51.

[68] U. Forstermann, J.S. Pollock, H.H. Schmidt, M. Heller and F. Murad. 1991. Calmodulindependent endothelium-derived relaxing factor/nitric oxide synthase activity is present in the particulate and cytosolic fractions of bovine aortic endothelial cells. Proc Natl Acad Sci U S A 88: 1788-92.

[69] H. Fukuda, T. Tomimatsu, N. Watanabe, J.W. Mu, M. Kohzuki, M. Endo, E. Fujili, T. Kanzaki and Y. Murata. 2001. Post-ischemic hypothermia blocks caspase-3 activation in the newborn rat brain after hypoxia-ischemia. Brain Res 910: 187-91.

[70] F.H. Giage. 2002. Neurogenesis in the adult brain. J Neurosci 22: 612-3.

[71] L. Garofalo, A. Ribeiro-da-Silva and A.C. Cuello. 1992. Nerve growth factor-induced synaptogenesis and hypertrophy of cortical cholinergic terminals. Proc Natl Acad Sci US A 89:2639-43.

[7.2] J. Garthwaite and C.L. Boulton. 1995. Nitric oxide signaling in the central nervous system. Annu Rev Physiol 57: 683-706.

[73] Y. Gavrieli, Y. Sherman and S.A. Ben-Sasson. 1992. Identification of programmed cell death in situ via speciffc labeling of nuclear DNA fragmentation. J Cell Bilol 119: 493-501.

[74] C.R. Gerfen. 1992. The neostriatal mosaic: multiple levels of compartmental organization. Trends Neurosci 15: 133-9.

[75] C.R. Gerfen, Wilson, C. J. 1996. The basal ganglia. In Swanson, L. W., Bjorklund, A., Hokfelt, T. eds. Handbook of Chemical Neuroanatomy: Integrated systems of the CNS, part III. Cerebellum, Basal Ganglia, Olfactory systems. Amsterdam, Elsevier Science B.V. 1996:
$371-468$.

[76] K.L. Gilby, J.N. Armstrong, R.W. Currie and H.A. Robertson. 1997. The effects of hypoxiaischemia on expression of c-Fos, $c-J u n$ and $\mathrm{Hsp} 70$ in the young rat hippocampus. Brain

[77] R. Gill, M. Soriano, K. Blomgren, H. Hagberg, R. Wybrecht, M.T. Miss, S. Hoefer, G. Adam, 
O. Niedlerhauser, J.A. Kemp and H. Loetscher. 2002. Role of caspase-3 activation in cerebrat ischemia-induced neurodegeneration in adult and neonatal brain. J Cereb Blood Flow Metab 22: 420-30.

[78] R.N. Goldberg, P. Moscoso, C.R. Bauer, F.L. Bloom, R.G. Curless, B. Burke and E. Bancalari. 1986. Use of barbiturate therapy in severe perinatal asphyxia: a randomized controlled trial. J Pediatr 109: 851-6.

[79] I.S. Goping, A. Gross, J.N. Lavoie, M. Ngulyen, R. Jemmerson, K. Roth, S.J. Korsmeyer and G.C. Shore. 1998. Regullated targeting of BAX to mitochondria. J Cell Biol 143: 207-15.

[80] E. Gould, A. Beylin, P. Tanapat, A. Reeves and T.J. Shors. 1999. Learning enhances adult neurogenesis in the hippocampal formation. Nat Neurosci 2: 260-5.

[81] E. Gould, A.J. Reeves, M.S. Graziano and C.G. Gross. 1999. Neurogenesis in the neocortex of adult primates. Science 286: 548-52.

[82] M.R. Grafe. 1994. Developmental changes in the sensitivity of the neonatal rat brain to hypoxic/ischemic injury. Brain Res 653: 161-6.

[83] D.R. Green and J.C. Reed. 1998. Mitochondria and apoptosis. Science 281: 1309-12.

[84] I. Grofova. 1975. The identification of striatal and pallidal neurons projecting to substantia nigra. An experimental study by means of retrograde axonal transport of horseradish peroxidase. Brain Res 91: 286-91.

[85] A. Gross, J. Jockel, M.C. Wei and S.J. Korsmeyer. 1998. Enforced dimerization of BAX results in its translocation, mitochondrial dysfunction and apoptosis. Embo $\mathrm{J}$ 17: 3878-85.

[86] J. Gross, I. Muller, Y. Chen, M. Elizallde, N. Leclere, M. Herrera-Marschitz and K. Andersson. 2000. Perinatal asphyxia induces region-specific long-term changes in mRNA levels of tyrosine hydroxylase and dopamine $\mathrm{D}(1)$ and $\mathrm{D}(2)$ receptors in rat brain. Brain Res $\mathrm{Mol}$ Brain Res 79: 110-7.

[87] J. Grotta, W. Clark, B. Coull, L.C. Pettigrew, B. Mackay, L.B. Goldstein, I. Meissner, D. Murphy and L. LaRue. 1995. Safety and tolerability of the glutamate antagonist CGS 19755 (Selfotel) in patients with acute ischemic stroke. Results af a phase lla randomized trial. Stroke 26: $602-5$.

[88] J. Guan, O.T. Miller, K.M. Waugh, D.C. McCarthy and P.D. Gluckman. 2001. Insulin-like growth factor- 1 improves somatosensory function and reduces the extent of cortical infarction and ongoing neuronal loss after hypoxia-ischemia in rats. Neuroscience 105: 299-306.

[89] A.J. Gunn. 2000. Cerebral hypothermia for prevention of brain injury following perinatal asphyxia. Curr Opin Pediatr 12: 111-5.

[90] A.J. Gunn, P.D. Gluckman and T.R. Gunn. 1998. Selective head cooling in newborn infants after perinatal asphyxia: a safety study. Pediatrics 102: 885-92.

[91] K. Gustafson, H. Hagberg, B.A. Bengtsson, C. Brantsing and J. Isgaard. 1999. Possible protective role of growth hormone in hypoxia-ischemia in neonatal rats. Pediatr Res 45: 318-23.

[92] R. Hakem, A. Hakem, G.S. Duncan, J.T. Henderson, M. Woo, M.S. Soengas, A. Elia, J.L. de la Pompa, D. Kagi, W. Khoo, J. Potter, R. Yoshida, S.A. Kaufman, S. W. Lowe, J.M. Penninger and T.W. Mak. 1998. Differential requirement for caspase 9 in apoptotic pathways in vivo. Cell 94: 339-52.

[93] B. Halliwell and J.M. Gutteridge. 1985. The importance of free radicals and catalytic metal ions in human diseases. Mol Aspects Med 8: 89-193.

[94] Y. Hashimoto, H. Kawatsura, Y. Shiga, S. Furukawa and T. Shigeno. 1992. Significance of nerve growth factor content levels after transient forebrain ischemia in gerbils. Neurosci Lett 139: 45-6.

[95] H. Hattori, A.M. Morin, P.H. Schwartz, D.G. Fujikawa and C.G. Wasterlain. 1989. Posthypoxic treatment with MK-801 reduces hypoxic-ischemic damage in the neonatal rat. Neurology 39: 713-8. 
[96] T. Hayakawa, Y. Hamada, T. Maihara, H. Hattorl and H. Mikawa. 1994. Phenytoin reduces neonatal hypoxic-ischemic brain damage in rats. Life Sici 54: 387-92.

[97] M.O. Hengartner. 2000. The biochemistry of apoptosis. Nature 407: 770-6.

[98] R.C. Henneberry. 1989. The role of neuronal energy in the neurotoxicity of excitatory amino acids. Neurobiol Aging 10:611-3; discussion 618-20.

[99] D. Heumann and G. Leuba. 1983. Neuronal death in the development and aging of the cerebral cortex of the mouse. Neuropathol Appl Neurobiol 9: 297-311.

[100] Y. Higuchi, H. Hattori, R. Hattori and K. Furusho. 1996. Increased neurons containing neuronal nitric oxide synthase in the brain of a hypoxic-ischemic neonatal rat model. Brain Dev 18: 369-75.

[101] A. Hill. 1991. Current concepts of hypoxic-ischemic cerebral injury in the term newborn. Pediatr Neurol 7: 3:7-25.

[102] H. Hoeger, M. Engelmann, G. Bernert, A. Seid,, H. Bubna-Littitz, W. Masgoeller, B. Lubec and $G$. Lubec. 2000. Long term neurological and behavioral effects of graded perinatal asphyxila in the rat. Life Sci 66: $947-62$.

[103] P.J. Horner and F.H. Gage. 2000. Regenerating the damaged central nervous system. Nature 407: 963-70.

[104] F.M. Hughes, Jr., R.B. Evans-Storms and J.A. Cidlowski. 1998. Evidence that non-Caspase proteases are required for chromatin degradation during apoptosis. Cell Death Differ 5 : $1017-27$.

[105] T. Ikeda, K. Mishima, T. Yoshikawa, K. Iwasaki, M. Fujiwara, Y.X. Xia and T. Ikenoue. 2001. Selective and long-term learning impairment following neonatal hypoxic-ischemic brain insult in rats. Behav Brain Res 118: 17-25.

[106] H. Ischiropoulos, L. Zhu, J. Chen, M. Tsai, J.C. Martin, C.D. Smith and J.S. Beckman. 1992. Peroxynitrite-mediated tyrosine nitration catalyzed by superoxide dismutase. Arch Biochem Biophys 298: 431-7.

[107] A. Ishida, S. Ishiwa, W.H. Trescher, W. Nakajima, M.S. Lange, M.E. Blue and M.V. Johnston. 2001. Delayed increase in neuronal nitric oxide synthase immunoreactivity in thalamus and other brain regions after hypoxic-ischemic injury in neonatal rats. Exp Neurol 168: 323-33.

[108] A. Ishida, W.H. Trescher, M.S. Lange and M.V. Johnston. 2001. Prolonged suppression of brain nitric oxide synthase activity by 7 -nitroindazole protects against cerebral hypoxicischemic injury in neonatal rat. Brain Dev 23: 349-54.

[109] M. Iwai, K. Sato, N. Omori, I. Nagano, Y. Manabe, M. Shoji and K. Abe. 2002. Three steps of neural stem cells development in gerbil dentate gyrus after transient ischemia. J Cereb Blood Flow Metab 22: $4111-9$.

[110] E.M. Jansen and W.C. Low. 1996. Long-term effects of neonatal ischemic-hypoxic brain injury on sensorimotor and locomotor tasks in rats. Behav Brain Res 78: 189-94.

[111] E.M. Jansen and W.C. Low. 1996. Quantitative analysis of contralateral hemisphere hypertrophy and sensorimotor performance in adult rats following unilateral neonatal ischemic-hypoxic brain injury. Brain Res 708: 93-9.

[112] W. Jiang, W. Gu, T. Brannstrom, R. Rosqvist and P. Wester. 2001. Cortical neurogenesis in adult rats after transient middle cerebral artery occlusion. Stroke 32: 1201-7.

[113] K. Jin, M. Minami, J.Q. Lan, X.O. Mao, S. Batteur, R.P. Simon and D.A. Greenberg.. 2001. Neurogenesis in dentate subgranuiar zone and rostral subventricular zone after focal cerebral ischemia in the rat. Proc Natl Acad Sci U S A 98: 4710-5.

[114] M. Johnson, G.R. Hanson, J.W. Gibb, J. Adair and F. Filloux. 1994. Effect of neonatal hypoxiaischemia on nigro-striatal dopamine receptors and on striatal neuropeptide $Y$, dynorphin $A$ and substance $P$ concentrations in rats. Brain Res Dev Brain Res 83: 109-18.

[115] J.M. Jurgensmeier, Z. Xie, Q. Deveraux, L. Ellerby, D. Bredesen and J.C. Reed. 1998. Bax 
directly induces release of cytochrome c from isolated mitochondria. Proc Natl Acad SoI U S A 95: 4997-5002.

[116] M.S. Kaplan and J.W. Hinds. 1977. Neurogenesis in the adult rat: electron microscopic analysis of light radioautographs. Science 197: 1092-4.

[117] C.D. Katsetos, E. Spandou, A. Legido, M.L. Taylor, S.A. Zanelli, J.P. de Chadarevian, S. Christakos, O.P. Mishra and M. Delivoria-Papadopoulos. 2001. Acute hypoxia-induced alterations of calbindin-D28k immunoreactivity in cerebellar Purkinje cells of the guinea pig fetus at term. J Neuropathol Exp Neurol 60: 470-82.

[118] J.F. Kerr, A.H. Wyllie and A.R. Currie. 1972. Apoptosis: a basic biological phenomenon with wide-ranging implications in tissue kinetics. Br J Cancer 26: 239-57.

[119] J.F.R.a.H. Kerr, B.V. 1991. Definition and incidence of apoptosis: A historical persperctive. In Tomei, L.D., Cope., F.O. (eds) Apoptosis, the molecular basis of cell death. Cold Spring Harbor, NY, Cold Spring Harbor Laboratory Press.

[120] B. Kirschenbaum, F. Doetsch, C. Lois and A. Alvarez-Buylla. 1999. Adult subventricular zone neuronal precursors continue to proliferate and migrate in the absence of the olfactory bulb. a Neurosci 19:2171-80.

[121] P.B. Kirschner, B.G. Jenkins, J.B. Schulz, S.P. Finkelstein, R.T. Matthews, B.R. Rosen and M.F. Beal. 1996. NGF, BDNF and NT-5, but not NT-3 protect against MPP+ toxicity and oxidative stress in neonatal animals. Brain Res $713: 178-85$.

[122] H. Kita and S.T. Kitai. 1988. Glutamate decarboxylase immunoreactive neurons in rat neostriatum: their morphological types and populations. Brain Res 447: 346-52.

[123] M. Klempt, N.D. Klempt and P.D. Gluckman. 1993. Hypoxia and hypoxia/ischemia affect the expression of insulin-like growth factor binding protein 2 in the developing rat brain. Brain Res Mol Brain Res 17: 347-50.

[124] C. Kohlhauser, S. Kaehler, W. Mosgoeller, N. Singewald, D. Kouvelas, H. Prast, H. Hoeger and B. Lubec. 1999. Histological changes and neurotransmitter levels three months following perinatal asphyxia in the rat. Life Sci 64: 2109-24.

[125] C. Kohlhauser, W. Mosgoller, H. Hoger and B. Lubec. 2000. Myelination deficits in brain of rats following perinatal asphyxia. Life Sci 67: 2355-68.

[126] S. Kothakota, T. Azuma, C. Reinhard, A. Klippel, ل. Tang, K. Chu, T.J. McGarry, M. W. Kirschner, K. Koths, D.J. Kwiatkowski and L. T. Williams. 1997. Caspase-3-generated fragment of gelsolin: effector of morphological change in apoptosis. Science 278: 294-8.

[127] G. Kroemer, N. Zamzami and S.A. Susin. 1997. Mitochondrial control of apoptosis. Immunol Today 18: $44-51$.

[128] C. Kuenzle, O. Baenziger, E. Martin, L. Thun-Hohenstein, M. Steinlin, M. Good S. Fanconi, E. Boltshauser and R.H. Largo. 1994. Prognostic value of early MR imaging in term infants with severe perinatal asphyxia. Neuropediatrics 25: 191-200.

[129] H.G. Kuhn, J. Winkler, G. Kempermann, L.J. Thal and F.H. Gage. 1997. Epidermal growth factor and fibroblast growth factor-2 have different effects on neural progenitors in the adult rat brain. $\mathbb{N}$ Neurosci $17: 5820-9$.

[130] K. Kuida, T.F. Haydar, C.Y. Kuan, Y. Gu, C. Taya, H. Karasuyama, M.S. Siu, P. Rakic and A.A. Flavell. 1998. Reduced apoptosis and cytochrome c-mediated caspase activation in mice lacking caspase 9. Cell 94: 325-37.

[131] K. Kuida, T.S. Zheng, S. Na, C. Kuan, D. Yang " H. Karasuyama, P. Rakic and R.A. Flavell. 1996. Decreased apoptosis in the brain and premature lethality in CPP32-deficient mice. Nature 384: 368-72.

[132] E. Kunkel-Bagden and B.S. Bregman. 1990. Spinal cord transplants enhance the recovery of locomotor function after spinal cord injury at birth. Exp Brain Res 81: 25-34.

[133] O. Labudova, E. Schuller, K. Yeghiazarjan, E. Kitzmueller, H. Hoeger, G. Lubec and B. Lubec. 
1999. Genes involved in the pathophysiology of perinatal asphyxia. Life Sci 64: 1831-8.

[134] S. Lamas, P.A. Marsden, G.K. LI. P. Tempst and T. Michel. 1992. Endothelial nitric oxide synthase: molecular cloning and characterization of a distinct constitutive enzyme isoform. Proc Nat Acad Sci U S A 89: 6348-52.

[135] M.G. Leggio, M. Molinari, P. Neri; A. Grazlano, L. Mandolesi and L. Petrosini. 2000. Representation of actions in rats: the role of cerebellum in learning spatial performances by observation. Proc Natl Acad Sci U S A 97: 2320-5.

[136] A. Legido, Parikh. N.A., Taylor, M.L., Kaleyias, J.K., de Chadarevian, J., Katsetos, C.D. 2002. Decreased immunoreactivity of Calbindin-D28K in cerebellar Purkinje cells of children with hypoxic/ischemic encephalopathy. Pediatric Academic Societies. Abstract nr. 2603.

[137] M. Leist and P. Nicotera. 1997. The shape of cell death. Biochem Biophys Res Commun 236: $1-9$.

[138] R.R. Leker and E. Shohami. 2002. Cerebral ischemia and trauma-different etiologies yet similar mechanisms: neuroprotective opportunities. Brain Res Brain Res Rev 39: 55-73.

[139] V. Lemaire, M. Koehi, M. Le Moal and D.N. Abrous. 2000. Prenatal stress produces learning deficits associated with an inhibition of meurogenesis in the hippocampus. Proc Natl Acad Sci U S A 97: $11032-7$.

[140] M. Levene. 1992. Role of excitatory amino acid antagonists in the management of birth asphyxia. Biol Neonate 62: 248-51.

[1.1] M.l. Levene, N.A. Gibson, A.C. Fenton. E. Papathoma and D. Barnett. 1990. The use of a calcium-channel blocker, nicardipine, for severely asphyxiated newborn infants.. Dev Med Chilld Neurol $32:$ 567-74.

[142] R. Levi-Montalcini. 1966. The nerve growth factor: its mode of action on sensory and sympathetic nerve cells. Harvey Lect $60: 217-59$.

[143] R. Levi-Montaicini. 1987. The nerve growth factor 35 years later. Science 237: 1154-62.

[144] R. Levi-Montalcini, S.D. Skaper, R. Dal Toso, L. Petrelli and A. Leon. 1996. Nerve growth factor: from neurotrophin to neurokine. Trends Neurosci 19: 514-20.

[145] S. Levine. 1960. Anoxic-ischemic encephalopathy in rats. Am. J. Pathol. 36: 1-17.

[146] S.W. Levison, C. Chuang, B.J. Abramson and J.E. Goldman. 1993. The migrational patterns and developmental fates of glial precursors in the rat subventricular zone are temporally regulated. Development 119: $611-22$.

[147] S.W. Levison and J.E. Goldman. 1993. Both oligodendrocytes and astrocytes develop from progenitors in the subventricular zone of postnatal rat forebrain. Neuron 10: 201-12.

[148] S.W. Levison, R.P. Rothstein, M.J. Romanko, M.J. Snyder, R.L. Meyers and S.J. Vannucci. 2001. Hypoxia/ischemia depletes the rat perinatal subventricular zone of oligodendrocyte progenitors and neural stem cells. Dev Neurosci 23: 234-47.

[149] P. Li, D. Nijhawan. I. Budihardjo, S.M. Srinivasula, M. Ahmad, E.S. Alnemri and X. Wang. 1997. Cytochrome $c$ and dATP-dependent formation of Apaf-1/caspase-9 complex initiates an apoptotic protease cascade. Cell 91: 479-89.

[150] P.A. Li, Q.P. He, H. Miyashita, W. Howllet, B.K. Siesjo and A. Shuaib. 1999. Hypothermia amelliorates ischemic brain damage and suppresses the release of extracellular amino acids in both normo- and hyperglycemic subjects. Exp Neurol 158: 242-53.

[151] C.F. Loidl, F. Capani, J.J. Lopez-Costa, A. Selvin-Testa, E.M. Lopez, J. Golldstein and J. Pecci-Saavedra. 1997. Short-term changes in NADPH-diaphorase reactivity in rat brain following perinatal asphyxia. Neuroprotective effects of cold treatment. Mol Chem Neuropathol 31: 301-16.

[152] C.F. Loidi, J. De Vente, M.M. van Ittersum, E.H. van Dijk "J.S. Vles, H.W. Steinbusch and C.E. Blanco. 1998. Hypothermia during or after severe perinatal asphyxia prevents increase in cyclic GMP-related nitric oxide levels in the newborn rat striatum. Brain Res $791: 303-7$. 
[153] C.F. Loidl, A.W. Gavilanes, E.H.Van Dijk, W. Vreuls, A. Blokland, J.S. Vles, H.W. Steinbusch and C.E. Blanco. 2000. Effects of hypothermia and gender on survival and behavior after perinatal asphyxia in rats. Physiol Behav 68: $263-9$.

[154] C.F. Loidl, M. Herrera-Marschitz, K. Andersson, Z.B. You, M. Goiny, W.T. O'Connor, $R$. Silveira, R. Rawal, B. Bjelke, Y. Chen and et al. 1994. Long-term effects of perinatal asphyxia on basal ganglia neurotransmitter systems studied with microdialysis in rat. Neurosci Lett 175: 9-12.

[155] C. Lois, J.M. Garcia-Verdugo and A. Alvarez-Buylla. 1996. Chain migration of neuronal precursors. Science 271: 978-81.

[156] H. Lorez, F. Keller, G. Ruess and U. Otten. 1989. Nerve growth factor increases in adult rat brain after hypoxic injury. Neurosci Lett 98: 339-44.

[157] C.J. Lowenstein, C.S. Glatt, D.S. Bredt and S.H. Snyder. 1992. Cloned and expressed macrophage nitric oxide synthase contrasts with the brain enzyme. Proc Natl Acad Sci U S A 89: $6711-5$.

[158] B. Lubec, M. Chiappe-Gutierrez, H. Hoeger, E. Kitzmueller and G. Lubec. 2000. Glucose transporters, hexokinase, and phosphofructokinase in brain of rats with perinatal asphyxia. Pediatr Res 47: 84-8.

[159] B. Lubec, O. Labudova H. Hoeger, L. Kirchner and G. Lubec. 2002. Expression of transcription factors in the brain of rats with perinatal asphyxia. Biol Neonate 81: 266-78.

[160] P. Meier, A. Finch and G. Evan. 2000. Apoptosis in development. Nature 407: 796-801.

[161] E. Mercuri, A. Guzzetta, L. Haataja, F. Cowan, M. Rutherford, S. Counsell, M. Papadimitriou, G. Cioni and L. Dubowitz. 1999. Neonatal neurological examination in infants with hypoxic ischaemic encephalopathy: correlation with MRI findings. Neuropediatrics 30: $83-9$.

[162] M.W. Miller. 1995. Relationship of the time of origin and death of neurons in rat somatosensory cortex: barrel versus septal cortex and projection versus local circuit neurons. J Comp Neurol 355: 6-14.

[163] J.C. Mills. 2001. Mechanisms underlying the hallmark features of the execution-phase of apoptosis. In Eds. Mattson, M.P., Estus, S., Rangnekar, V.M. Advances in Cell aging and gerontology: Programmed cell death. Volume 1.: 1-38.

[164] O.P. Mishra and M. Delivoria-Papadopoulos. 1999. Cellular mechanisms of hypoxic injury in the developing brain. Brain Res Bull 48: 233-8.

[165] C.P. Montoya, L.J. Campbell-Hope, K.D. Pemberton and S.B. Dunnett. 1991. The "staircase test": a measure of independent forelimb reaching and grasping abilities in rats. J Neuroscl Methods 36: 219-28.

[166] S.M. Mooney and M.W. Miller. 2000. Expression of bcl-2, bax, and caspese -3 in the brain of the developing rat. Brain Res Dev Brain Res 123: 103-17.

[167] R. Morris. 1984. Developments of a water-maze procedure for studying spatial learning in the rat. J Neurosci Methods 11: 47-60.

[168] T. Nakagawa and J. Yuan. 2000. Cross-talk between two cysteine protease families. Activation of caspase-12 by calpain in apoptosis. J Cell Biol 150: 887-94.

[169] W. Nakajima, A. Ishida, M.S. Lange, K.L. Gabrielson, M.A. Wilson, L.J. Martin, M.E. Blue and M.V. Johnston. 2000. Apoptosis has a prolonged role in the neurodegeneration after hypoxic ischemia in the newborn rat. J Neurosici 20: 7994-8004.

[170] Y. Nakamura, T. Nakashima, S. Fukuda, H. Nakashima and T. Hashimoto. 1986. Hypoxicischemic brain lesions found in asphyxiating neonates. Acta Pathol Jpn 36: 551-63.

[171] K. Nakashima and M.M. Todd. 1996. Effects of hypothermia on the rate of excitatory amino acid release after ischemic depolarization. Stroke 27: 913m $\mathrm{B}$.

[172] D.W. Nicholson, A. Ali, N.A. Thornberry, J.P. Vaillancourt, C.K. Ding, M. Gallant, Y. Gareau, P.R. Griffin, M. Labelle, Y.A. Lazebnik and et al. 1995. Identification and inhibition of the ICE 
CED-3 protease necessary for mammalian apoptosis. Nature $376: 37-43$.

[173] F.J. Northington, D.M. Ferriero, D.L. Flock and L.J. Martin. 2001. Delayed neurodegeneration in neonatal rat thalamus after hypoxia-ischemia is apoptosis. I Neurosci 21: 1931-8.

[174] F.J. Northington, D.M. Ferriero, E.M. Graham, R.J. Traystman and L.J. Martin. 2001. Early Neurodegeneration after Hypoxia-lschemia in Neonatal Rat Is Necrosis whille Delayed Neuronal Death Is Apoptosis. Neurobiol Dis 8: 207-19.

[175] F.J. Northington, D.M. Ferriero and L.J. Martin. 2001. Neurodegeneration in the thalamus following neonatal hypoxia-ischemia is programmed cell death. Dev Neurosci 23: 186-91.

[176] C. Nyakas, B. Buwalda and P.G. Luiten. 1996. Hypoxia and brain development. Prog Neurobiol 49: 1-51.

[177] J.R. O'Kusky, $P_{\text {. }}$ Ye and A.J. D'Ercole. 2000. Insulin-like growth factor-1 promotes neurogenesis and synaptogenesis in the hippocampal dentate gyrus during postnatal development. J Neurosci 20: 8435-42.

[178] C. Paimer and R.C. Vannucci. 1993. Potential new therapies for perinatal cerebral hypoxiaischemia. Clin Perinatol 20: 411-32.

[179] J.F. Pasternak, T.A. Predey and M.A. Mikhael. 1991. Neonatal asphyxia: vulnerability of basal ganglia, thalamus, and brainstem. Pediatr Neurol 7: 147-9.

[180] C.M. Payne and D.W. Cromey. 1992. An image analysis workstation designed for multiple users: application of quantitative digital imaging techniques to electron microscopy. Ultrastruct Pathol 16: 147-54.

[181] V. Pencea, K.D. Bingaman, S.J. Wiegand and M.B. Luskin. 2001. Infusion of brain-derived neurotrophic factor into the lateral ventricle of the adult rat leads to new neurons in the parenchyma of the striatum, septum, thalamus, and hypothalamus. J Neurosci 21: 670617.

[182] D.A. Peterson. 2002. Stem cells in brain plasticity and repair. Curr Opin Pharmacol 2: 34-42.

[183] L. Petrosini, M.G. Leggio and M. Molinari. 1998. The cerebellum in the spatial problem solving: a co-star or a guest star? Prog Neurobiol 56: $191-210$.

[184] L. Petrosini, M. Molinari and M.E. Dell'Anna. 1996. Cerebellar contribution to spatial event processing: Morris water maze and T-maze. Eur J Neulrosci 8: 1882-96.

[185] C. Portera-Cailliau, D.L. Price and L.J. Martin. 1997. Excltotoxic neuronal death in the immature brain is an apoptosis-necrosis morphological continuum. J Comp Neurol 378: 7087.

[186] E.R. Pschirrer and E.R. Yeomans. 2000. Does asphyxia cause cerebral palsy? Semin Perinatol 24: 215-20.

[187] M. Puka-Sundwall, B. Gajkowska, M. Cholewinski, K. Blomgren, J.W. Lazarewicz and H. Hagberg. 2000. Subcellular distribution of calcium and uitrastructural changes after cerebral hypoxia-ischemia in immature rats. Brain Res Dev Brain Res 125: 31-41.

[188] M.R. Pulera, L.M. Adams, H. Liu, D.G. Santos, R.N. Nishimura, F.Yang "G.M. Cole and C.G. Wasterlain. 1998. Apoptosis in a neonatal rat model of cerebral hypoxia-ischemia. Stroke 29: $2622-30$

[189] S. Rabizadeh, J. Oh, L. T. Zhong, J. Yang, C.M. Bitler, L.L. Butcher and D. E. Bredesen. 1993. Induction of apoptosis by the low-affinity NGF receptor. Science 261: 345-8.

[190] R. Radi, J.S. Beckiman, K.M. Bush and B.A. Freeman. 1991. Peroxynitrite oxidation of sulfhydryls. The cytotoxic potential of superoxide and nitric oxide. J Biol Chem 266: 4244 50.

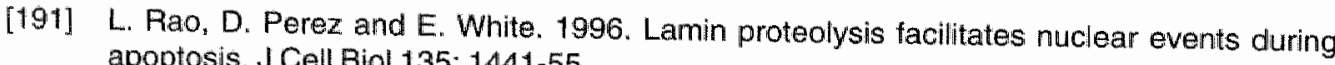
apoptosis. J Cell Biol 135: 1441-55.

[192] J.C. Reed. 1994. Bcl-2 and the regulation of programmed cell death. J Cell Biol 124: 1-6.

[193] J.C. Reed. 1997. Double identity for proteins of the Bol-2 family. Nature 387: 773-6. 
[194] J.C. Reed, J.M. Jurgensmeier and S. Matsuyama. 1998. Bcl-2 family proteins and mitochondria. Biochim Biophys Acta 1366: 127-37.

[195] A. Riccio, S. Ahn, C.M. Davenport, J.A. Blendy and D.D. Ginty. 1999. Mediation by a C.REB family transcription factor of NGF-dependent survival of sympathetic neurons. Science 286: $2358-61$.

[196] J.E. Rice, 3rd, R.C. Vannucci and J.B. Brierley. 1981. The influence of immaturity on hypoxicischemic brain damage in the rat. Ann Neurol 9: 131-41.

[197] E.H. Roland, K. Poskitt, E. Rodriguez, B.A. Lupton and A. Hill. 1998. Perinatal hypoxicischemic thalamic injury: clinical features and neuroimaging. Ann Neurol 44: 161-6.

[198] T. Roohey, T.N. Raju and A.N. Moustogiannis. 1997. Animal models for the study of perinatal hypoxic-ischemic encephalopathy: a critical analysis. Early Hum Dev 47: 115-46.

[199] J.P. Rossiter, L.L. Anderson, F.Yang and G.M. Cole. 2002. Caspase-3 activation and caspaselike proteolytic activity in human perinatal hypoxic-ischemic brain injury. Acta Neuropathol (Berl) 103: 66-73

[200] T. Rudel and G.M. Bokoch. 1997. Membrane and morphological changes in apoptotic cells regulated by caspase-mediated activation of PAK2. Science 276: 1571-4.

[201] H. Sakahira, Y. Takemura and S. Nagata. 2001. Enzymatic active site of caspase-activated DNase (CAD) and its inhibition by inhibitor of CAD. Arch Biochem Biophys 388: 91-9.

[202] U. Sartorius, 1. Schmitz and P.H. Krammer, 2001. Molecular mechanisms of death-receptormediated apoptosis. Chembiochem 2: 20-9.

[203] H. Schneider, L. Ballowitz, H. Schachinger, F. Hanefeld and J.U. Droszus. 1975. Anoxic encephalopathy with predominant involvement of basal ganglia, brain stem and spinal cord in the perinatal period. Report on seven newborns. Acta Neuropathol (Berl) 32: 287-98.

[204] P.H. Schwartz, W.F. Massarweh, H.V. Vinters and C.G. Wasterlain. 1992. A rat model of severe neonatal hypoxic-ischemic brain injury. Stroke 23: 539-46.

[205] R. Seidl, S. Stockler-Ipsiroglu, B. Rolinski, C. Kohthauser, K.R. Herkner, B. Lubec and G. Lubec. 2000. Energy metabolism in graded perinatal asphyxia of the rat. Life Sci 67:421-35.

[206] R. Sgonc and J. Gruber. 1998. Apoptosis detection: an overview. Exp Gerontol 33: 525-33.

[207] E.S. Sirimanne, R.M. Blumberg, D. Bossano, M. Gunning, A.D. Edwards, P.D. Gluckman and C.E. Williams. 1996. The effect of prolonged modification of cerebral temperature on outcome after hypoxic-ischemic brain injury in the infant rat. Pediatr Res 39: 591-7.

[208] S.V. Sizonenko, E.S. Sirimanne, C.E. Williams and P.D. Gluckman. 2001. Neuroprotective effects of the N-terminal tripeptide of IGF-1, glycine-proline-glutamate, in the immature rat brain after hypoxic-ischemic injury. Brain Res 922: 42-50.

[209] J.a.C. Soghomonian, M. 2000. GABA in the basal ganglia. In: Martin, D.L., Olsen, R.W. eds. GABA in the nervous system: the view at fifty years. New York: Lippincott Williams \& Wilkins, 2000: 265-291.

[210] J.S. Soul, R.L. Robertson, A.A. Tzika, A.J. du Plessis and J.J. Volpe. 2001. Time course of changles in diffusion-weighted magnetic resonance imaging in a case of neonatal encephalopathy with defined onset and duration of hypoxic-ischemic insult. Pediatrics 108 : $12 \| 1-4$.

[211] R. Spreafico, C. Frassoni, P. Arcelli, M. Selvaggio and S. De Biasi. 1995. In situ labeling of apoptotic cell death in the cerebral cortex and thalamus of rats during development. $J$ Comp Neurol 363: 281-95.

[212] M.K. Squier, A.C. Miller, A.M. Malkinson and J.J. Cohen. 1994. Calpain activation in apoptosis. J Cell Physiol 159: 229-37.

[213] J.S. Stamler, D.I. Simon, J.A. Osborne, M.E. Mullins, O. Jaraki, T. Michel, D.J. Singel and J. Loscalzo. 1992. S-nitrosylation of proteins with nitric oxide: synthesis and characterization of biologically active compounds. Proc Natl Acad Sci U S A 89:444-8. 
[214] S.A. Susin, H.K. Lorenzo, N. Zamzami, L Narzo, B.E. Snow, G.M. Brothers, J. Mangion, E. Jacotot, P. Costantini, M. Loeffler, N. Larochette, D.P. Goodlett, R. Aebersold, D. P. Siderovski, J.M. Penninger and G. Kroemer. 1999. Molecular characterization of mitochondrial apoptosisinducing factor. Nature $397: 441-6$.

[215] N.W. Svenningsen, G. Blennow, M. Lindroth, P.O. Gaddlin and H. Ahistrom. 1982. Brainorientated intensive care treatment in severe neonatal asphyxia. Effects of phenobarbitone protection. Arch Dis Child 57: 176-83.

[216] C. Szabo and V.L. Dawson, 1998. Role of poly(ADP-ribose) synthetase in inflammation and ischaemia-reperfusion. Trends Pharmacol Sci 19: 287-98.

[217] K. Takasawa, K. Kitagawa, Y. Yagita, T. Sasaki, S. Tanaka, K. Matsushita, T. Ohstuki, T. Miyata, H. Okano, M. Hori and M. Matsumoto. 2002. Increased proliferation of neural progenitor cells but reduced survival of newborn cells in the contralateral hippocampus after focal cerebral ischemia in rats. J Cereb Blood Flow Metab 22: 299-307.

[218] S. Takayama, T. Sato, S. Krajewsiki, K. Kochel, S. Irie, J.A. Millan and J.C. Reed. 1995. Cloning and functional analysis of BAG-1: a novel Bcl-2-binding protein with anti-cell death activity. Cell 80: 279-84.

[219] R.V. Talanian, C. Quinlan, S. Trautz, M.C. Hackett, J.A. Mankovich, D. Banach, T. Ghayur, K.D. Brady and W.W. Wong. 1997. Substrate specificities of caspase family proteases. J Biol Chem 272: $9677-82$.

[220] M. Tanaka, T. Momoi and T. Marunouchi. 2000. In situ detection of activated caspase-3 in apoptotic granule neurons in the dleveloping cerebellum in slice cultures and in vivo. Brain Res Dev Brain Res 121: 223-8.

[221] P. Tanapat, L.A. Galea and E. Gould. 1998. Stress inhibits the proliferation of granule cell precursors in the developing dentate gyrus. Int J Dev Neurosci 16: 235-9.

[222] P. Tanapat, N.B. Hastings, A.J. Reeves and E. Gould. 1999. Estrogen stimulates a transient increase in the number of new neurons in the dentate gyrus of the adult female rat. $J$ Neurosci 19: 5792-801.

[223] D.L. Taylor, H. Mehmet, E.B. Cady and A.D. Edwards. 2002. Improved neuroprotection with hypothermia delayed by 6 hours following cerebral hypoxia-ischemia in the 14-day-old rat. Pediatr Res 51: 13-9.

[224] C.E. Teunissen, H.W. Steinbusch, M. Angevaren, M. Appels, C. de Bruijn, J. Prickaerts and J. de Vente. 2001. Behavioural correlates of striatal glial fibrillary acidic protein in the 3nitropropionic acid rat model: disturbed walking pattern and spatial orientation. Neuroscience 105: 153-67.

[225] N.A. Thomberry and Y. Lazebnik. 1998. Caspases: enemies within. Science 281: 1312-6.

[226] T. Tomimatsu, H. Fukuda, M. Endo, N. Watanabe, J. Mu, M. Kohzuki, E. Fujii, T. Kanzaki and Y. Murata. 2001. Effects of hypothermia on neonatal hypoxic-ischemic brain injury in the rat: phosphorylation of Akt, activation af caspase-3-like protease. Neurosci Lett 312: 21-4.

[227] T. Tomimatsu, H. Fukuda, M. Endoh, J. Mu, N. Watanabe, M. Kohzuki, E. Fujii, T. Kanzaki, K. Oshima, K. Doi, T. Kubo and Y. Murata. 2002. Effects of neonatal hypoxic-ischemic brain injury on skilled motor tasks and brainstem function in adult rats. Brain Res 926: 108-17.

[228] T. Toyoda, S. Suzuki, N.F. Kassell and K.S. Lee. 1996. Intraischemic hypothermia attenuates neutrophil infiltration in the rat neocortex after focal ischemia-reperfusion injury. Neurosurgery

[229] W.H. Trescher, S. Ushiwa and M.V. Johnston. 1997. Brief post-hypoxic-ischemic hypothermia markedly delays neonatal brain injury. Brain Dev 19: 326-38.

[230] F. Van Bel, M. Shadid, R.M. Moison, C.A. Dorrepaal, J. Fontijn, L. Monteiro, M. Van De Bor and H.M. Berger. 1998. Effect of allopurinol on postasphyxial free radical formation, cerebral hemodynamics, and electrical brain activity. Pediatrics 101: 185-93. 
[231] W.D. Van de Berg, C. Schmitz, H.W. Steinbusch and C.E. Blanco. 2002. Perinatal asphyxia induced neuronal loss by apoptosis in the neonatal rat striatum: a combined TUNEL and stereological study. Exp Neurol 174: 29-36.

[232] H. van Praag, G. Kempermann and F.H. Gage. 2000. Neural consequences of environmental enrichment. Nat Rev Neurosci 1: 191-8.

[233] H. van Praag, A.F. Schinder, B.R. Christie "N. Toni, T.D. Palmer and F.H. Gage. 2002. Functional neurogenesis in the adult hippocampus. Nature 415: 1030-4.

[234] S.R. Vincent. 2000. Histochemistry of nitric oxide synthase in the central nervous system. In Handbook of Chemical Neuroanatomy 17: 19-49.

[235] J.J. Volpe. 1998. Brain injury in the premature infant: overview of clinical aspects, neuropathology, and pathogenesis. Semin Pediatr Neurol 5: 135-51.

[236] J.J. Volpe. 2001. Perinatal brain injury: from pathogenesis to neuroprotection. Ment Retard Dev Disabil Res Rev 7: 56-64.

[237] J. Voogd and M. Glickstein. 1998. The anatomy of the cerebellum. Trends Neurosci 21: 3705.

[238] J. Voogd, Jaarsma, D., Marani, E. 1996. The cerebelturn: chemoarchitecture and anatomy. In: Swanson, L.W., Bjorklund, A., Hokfelt, T. Handbook of chemical Neuroanatomy: Integrated systems of the CNS, Part III. Cerebellum, Basal Ganglia, Olfactory system. Amsterdam, Elsevier 1996: 1-369.

[239] B.P.Wagner, J. Nedelcu and E. Martin. 2002. Delayed postischemic hypothermia improves long-term behavioral outcome after cerebral hypoxia-ischemia in neonatall rats. Pediatr Res 51: $354-60$.

[240] C.L. Wagner, D.J. Eicher, L.D. Katikanemi, E. Barbosa and K.R. Holden. 1999. The use of hypothermia: a role in the treatment of neonatal asphyxia? Pediatr Neurol 21: 429-43.

[241] J.P. Wagner, I.B. Black and E. DiCicco-Bloom. 1999. Stimulation of neonatal and adult brain neurogenesis by subcutaneous injection of basic fibroblast growth factor. J Neurosci 19: 6006-16.

[242] H.G. Wang, U.R. Rapp and J.C. Reed. 1996. Bcl-2 targets the protein kinase Raf-1 to mitochondria. Cell 87: 629-38.

[243] C.G. Wasterlain, L.M. Adams, P.H. Schwartz, H. Hattori, R.D. Sofia and J.K. Wichmann. 1993. Posthypoxic treatment with felbamate is neuroprotective in a rat model of hypoxiaischemia. Neurology 43: 2303-10.

[244] R. Weitzdoerfer, N. Gerstl, H. Hoeger, W. Mosgoeller, W. Dreher, E. Engidawork, J. Overgaard-Larsen and B. Lubec. 2002. Long-term sequelae of perinatal asphyxia in the aging rat. Cell Mol Life Sci 59: 519-26.

[245] K.A. Wood and R.J. Youle. 1994. Apoptosis and free radicals. Ann NY Acad Sci 738: 400-7.

[246] X.Y. Xia, T. Ikeda, A. Ota, Y.X. Xia, H. Sameshima, T. Ikenoue and K. Toshimori. 1999. Heat shock protein 72 expression and microtubule-associated protein 2 disappearance after hypoxia-ischemia in the developing rat brain. Am J Obstet Gynecol 180: 1254-62.

[247] Q. Xiao and V.M. Nikodem. 1998. Apoptosis in the developing cerebellum of the thyroid hormone deficient rat. Front Biosci 3: A.52-7.

[248] Q.W. Xie, H.J. Cho, J. Calaycay, R.A. Mumford, K.M. Swiderek, T.D. Lee, A. Ding. T. Troso and C. Nathan. 1992. Cloning and characterization of inducible nitric oxide synthase from mouse macrophages. Science 256: 225-8.

[249] Y. Yagita, K. Kitagawa, T. Ohtsuki, K. Takasawa, T. Miyata, H. Okano, M. Hori and M. Matsumoto. 2001. Neurogenesis by progenitor cells in the ischemic adult rat hippocampus. Stroke 32: 1890-6.

[250] J. Yang, X. Liu, K. Bhalla, C.N. Kim, A.M. lbrado, J. Cai, T.I. Peng, D.P. Jones and X. Wang. 1997. Prevention of apoptosis by Bcl-2: release of cytochrome c from mitochondria blocked. Science 275: 1129-32. 
[251] J. Yelnik, C. Francois, G. Percheron and D. Tande. 1991. Morphological taxonomy of the neurons of the primate striatum. J Comp Neurol 313:273-94.

[252] H. Yoshida, Y.Y. Kong, R. Yoshida, A.J. Ellia, A. Hakem, R. Hakem, J.M. Penninger and T.W. Mak. 1998. Apalt is required for mitochondrial pathways of apoptosis and brain development. Cell $94: 739-50$.

[253] R.S.Young, J. Kolonich, C.L. Woods and S.K. Yagel. 1986. Behavioral performance of rats following neonatal hypoxia-ischemia. Stroke 17: 1313-6.

[254] J. Yuan and B.A. Yankner. 2000. Apoptosis in the nervous system. Nature 407: 802-9.

[255] G.D. Zeevalk and W.J. Nicklas. 1990. Action of the anti-ischemic agent ifenprodil on Nmethyl-D-aspartate and kainate-mediated excitotoxicity. Brain Res. 522: 135-9.

[256] K.C. Zimmermann, C. Bonzon and D.R. Green. 2001. The machinery of programmed cell death. Pharmacol Ther 92: 57-70.

[257] J.A. Zivin. 1997. Neuroprotective therapies in stroke. Drugs 54 Suppl 3: 83-8; discussion 88-9.

[258] M.H. Zornow. 1995. Inhibition of glutamate release: a possible mechanism of hypothermic neuroprotection. J Neurosurg Anesthesiol 7: 148-51. 


\section{Use of cryostat sections from snap- frozen nervous tissue for combining stereological estimates with histological, cellular, or molecular analyses on adjacent sections}

C. Schmitz, M. Dafotakis, H. Heinsen, K. Mugrauer, A. Niesel, G.J. Popken, M. Stephan, W.D.J. Van de Berg, S. von Hörsten, H. Korp J Chem Neuroanatomy 2000;20:21-29 


\section{Abstract}

Adequate tissue preparation is essential for both modern stereological and immunohistochemical investigations. However, combining these methodologies in a single study presents a number of obstacles pertaining to optimal histological preparation. Tissue shrinkage and loss of nuclei/nucleoli from the unprotected section surfaces of unembedded tissue used for immunohistochemistry may be problematic with regard to adequate stereological design. In this study, frozen cryostat sections from hippocampal and cerebellar regions of two rat strains and cerebellar and cerebral regions from a human brain were analyzed to determine the potential impact of these factors on estimates of neuron number obtained using the optical disector. Neuronal nuclei and nucleoli were clearly present in thin sections of snap-frozen rat $(3 \mu \mathrm{m})$ and human $(6 \mu \mathrm{m})$ tissue, indicating that neuronal nuclei/nucleoli are not unavoidably lost from unprotected section surfaces of unembedded tissue. In order to quantify the potential impact of any nuclear loss, optical fractionator estimates of rat hippocampal pyramidal cells in areas CA1-3 and cerebellar granule and Purkinje cells were made using minimal $(1 \mu \mathrm{m})$ upper guard zones. Estimates did not differ from data reported previously in the literature. This data indicates that cryostat sections of snap-frozen nervous tissue may successfully be used for estimating total neuronal numbers using optical disectors. 


\section{Introduction}

The use of thick cryostat sections (up to $700 \mu \mathrm{m}$ ) and thick methacrylate sections (up to 70 $\mu \mathrm{m})$ has become routine for estimating total neuronall numbers using the optical disector (concerning the use of cryostat sections see $[9,25,26]$; concerning the use of methacrylate sections see $[4,13,30])$. Methacrylate sections have an advantage over cryostat sections in that they show less shrinkage in section thickness during histological processing (in the following text, shrinkage in section thickness will be addressed by a thickness shrinkage factor. By definition, a thickness shrinkage factor of 1 means that there is no shrinkage in section thickness, whereas a thickness shrinkage factor of, say, 0.33 means that the original section thickness was three times the thickness after histological processing). The thickness shrinkage factor for methacrylate sections has been reported as between 1 [27] and 0.78 [17], while the thickness shrinkage factor for cryostat sections of formalin-fixed nervous tissue has been found to be between 0.8 [9] and 0.32 [24]. However, there are two major disadvantages of thick methacrylate sections with respect to the application of the optical disector. First, methacrylate sections may show a substantially lower density of neurons in the core than in the upper and lower margins of these sections due to compression of the tissue edges by the knife blade during sectioning [7,29]. Second, it is impossible to label specimens adequately using immunohistochemical techniques or fluorescent markers since the methacrylate resin is not removed from the cut specimens $[1,19]$.

In the cerebellar nucleus dentatus a loss of all nucleoli of neurons in the upper $5 \mu \mathrm{m}$ (related to the original section thickness) and almost all nucleoli in the bottom $10 \mu \mathrm{m}$ has been reported in $100 \mu \mathrm{m}$ thick vibratome sections of a human brain fixed for approximately 10 years in buffered formalin [1]. The authors suggested that cells in the unprotected section surfaces of the unembedded tissue may have lost their nuclei when hit by the knife.

Loss of nucleoli or even nuclei at the sections edges may seriously affect reliability of neuron counts. In order to address this problem, Andersen and Gundersen (1999) suggest that when using the optical disector for counting neurons, one must respect rather large guard zones, sometimes on the order of the cell's diameter, at the upper and lower surface of thick sections of unembedded tissue [1]. However, use of such large guard zones is difficult when combining stereology and immunohistochemistry. For example, in a confocal laser microscopy optical disector study quantifying GABAergic neurons in the mouse hippocampus, Jimno et al. found that GAD67 immunostaining for somatic profiles reliably penetrated the section $14 \mu \mathrm{m}$ from the surface but was markedly decreased at $18 \mu \mathrm{m}$ from the surface (perfusion-fixed brains; 50 $\mu \mathrm{m}$ thick vibratome sections; mean thickness shrinkage factor $=0.70)$ [.11]. Therefore, they used optical sections $2 \mu \mathrm{m}$ from the upper surfaces as look-up sections of the optical disectors, and those $4-12 \mu \mathrm{m}$ from the surfaces as reference sections. The authors emphasized that there were no appreciable differences in the numbers of immunoreactive somatic profiles between the optical sections near the upper surfaces and those at $14 \mu \mathrm{m}$ from the upper surfaces of the investigated sections.

It cannot be excluded that the loss of neuronal muclei or nucleoli across unprotected section surfaces reported by Andersen and Gundersen (1999) may also occur in sections of snap- 
frozen nervous tissue. However, dependent on the study objectives, it may be most appropriate to use snap-frozen sections for counting neurons with the optical disector. For example, several studies in rats have demonstrated that postnatal experiences such as handling or maternal deprivation alter glucocorticoid mRNA receptor expression levels $[18,28]$. Since modulation of peripheral glucocorticoid levels might be associated with glucocorticoid-induced alterations of neuronal numbers in the central nervous system, it might be worthwhile to estimate neuronal numbers on adjacent slices of such brains. The most prudent experimental design would be to use brains that have been snap-frozen immediately after decapitation and apply modern stereological techniques to sections corresponding with the in situ hybridization sections. Therefore, it is the aim of the present study to evaluate whether or not there is a general loss of nuclei or nucleoli in the upper $5 \mu \mathrm{m}$ (related to the original section thickness) of serial cryostat sections of snap-frozen rat or human brains. Furthermore, we addressed the question whether or not sections of snap-frozen rat brain may be used for counting neurons using the optical disector. To do this, we estimated the mean total number of hippocampal pyramidal cells and cerebellar granule and Purkinje cells in the brains of adult rats using cryostat sections and the optical fractionator, deliberately placing the top of the counting frames only $1 \mu \mathrm{m}$ below the upper surface of the sections. The results were compared with data reported in the literature.

\section{Materials and methods}

\section{Experiment 1}

Experiment 1 was carried out using the brains of a 7 -month-old male Lewis rat (L), a 1 month-old male Wistar rat (W), and a 29-year-old male human (H) who died of a drug overdose but was without known neurological or psychiatric disorders. Investigations were approved by the Ethical Boards of Hannover District Government (Hannover, Germany) (L), the Studiecentrum voor Kernenergie / Centre d'Étude de l'Énergie Nucléaire (CENSCK; Mol, Belgium) (L), and the Faculty of Medicine at the University of Wuerzburg (Wuerzburg, Germany).

After decapitation of the Lewis rat, the brain was quickly removed from the skull and immediately frozen in a bucket containing 2-methylbutane kept at $-50^{\circ} \mathrm{C}$ on dry ice. The brain was stored at $-80^{\circ} \mathrm{C}$, then moved to a freezer at $-20^{\circ} \mathrm{C}$ fifteen hours before cutting. A parasagittal cut was made in the right brain half approximately $3 \mathrm{~mm}$ from the mediosagittal line. The resulting surface was used to mount the brain on the specimen holder of a cryostat (HM500 OMV, Microm, Germany) using Tissue-Tek (Sakura, Japan; temperature in the box $-15^{\circ} \mathrm{C}$, temperature of the specimen holder $-10^{\circ} \mathrm{C}$ ). Serial sagittal sections of varying thickness $(3 \mu \mathrm{m}$ to $100 \mu \mathrm{m})$ of the left hemisphere were cut using disposable 76 -mm microtome blades (Feather, Japan). Sections were thaw-mounted onto gelatinized glass slides (room temperature, RT) and dried on a heating plate for $1 \mathrm{~h}$ at $37^{\circ} \mathrm{C}$. Sections were 
fixed for one hour in acetone (RT), stored for $20 \mathrm{~min}$ in $\mathrm{Na}$-acetate buffer $(0.2 \mathrm{M} ; \mathrm{RT})$, stained

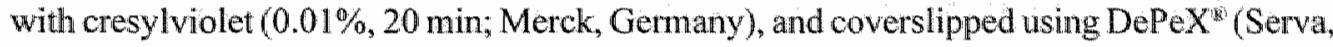
Germany).

The Wistar rat (W) was anesthetized with chloralhydrate $(0.005 \mathrm{ml} / \mathrm{g}$ body weight $[\mathrm{BW}]$, i.p.) and perfused with $0.1 \%$ procaine in Ringer's solution $\left(37^{\circ} \mathrm{C} ; 20\right.$ s) followed by $4 \%$ formalin in saline ( $\mathrm{RT}, \mathrm{pH} 7.2 ; 10 \mathrm{~min}$ ). After opening the skull, the head was fixed for approximately $24 \mathrm{~h} \mathrm{in} 4 \%$ formalin at $4^{\circ} \mathrm{C}$. The brain was removed, halved in the mediosagittal line, and further fixed for 10 days in $4 \%$ formalin at $4^{\circ} \mathrm{C}$. After rinsing the left half of the brain for 12 $\mathrm{h}$ in tap water, it was cryoprotected using increasing sucrose $0.1 \mathrm{M}$ Tris buffer solutions $(\mathrm{pH}$ $7.4 ; 4^{\circ} \mathrm{C} ; 10 \%$ for $24 \mathrm{~h}, 20 \%$ for $24 \mathrm{~h}, 30 \%$ for $48 \mathrm{~h}$ ). The brain half was frozen in Tissue-Tek and cut into serial sagittal sections of varying thicknesses $(6 \mu \mathrm{m}$ to $100 \mu \mathrm{m})$ on a cryostat (temperature in the box $-20^{\circ} \mathrm{C}$, temperature of the specimen holder $-15^{\circ} \mathrm{C}$ ). Here we were not able to obtain thinner sections with adequate gross morphology. Sections were thaw-mounted onto gelatinized glass slides (RT), dried for $1 \mathrm{~h}$ at $37^{\circ} \mathrm{C}$ on a heating plate, and stored for $24 \mathrm{~h}$ at $37^{\circ} \mathrm{C}$ in a thermostatically-controlled oven. Sections were degreased with Triton $\mathrm{X}-100$ (Merck, Germany; $0.025 \%, 20 \mathrm{~min})$, stained with cresylviolet $(0.01 \%, 20 \mathrm{~min}$ ), and coverslipped using DePeX

The human brain was immersion-fixed in $10 \%$ aqueous formalin solution for 24 months. Blocks of cerebral and cerebellar cortex approximately $1.5 \mathrm{~cm}^{3}$ were cut from the brain. These blocks were processed as described for the Wistar rat brain, starting with the $12 \mathrm{~h}$ tap water rinse. Serial sections of varying thicknesses $(5 \mu \mathrm{m}$ to $100 \mu \mathrm{m})$ were collected. We were not able to obtain thinner sections with adequate gross morphology. In addition to staining with cresylviolet, some sections were stained with gallocyanin $(0.15 \%, 24 \mathrm{~h})$.

All sections were examined for the presence of neuronal nuclei and nucleoli using a Leitz Ortholux II light microscope (Leitz, Germany) and a 40x objective (Leitz NPL FL 40, NA = 0.70 ) and a 100x objective (Leitz NPL Fluotar 100, oil, NA = 1.32). Representative photomicrographs were taken from hippocampal area $\mathrm{CA} 3(\mathrm{~L}, \mathrm{~W})$, cerebellar cortex $(\mathrm{L}, \mathrm{W}$, $\mathrm{H})$, and cerebral cortex layer $\mathrm{V}(\mathrm{H})$ of the thinnest sections for each subject.

\section{Experiment 2}

Experiment 2 was carried out using two groups of rats. The first group consisted of six $7-$ month-old Fischer 344 rats, and the second group of six Lewis rats of the same age. Investigations were approved by the Ethical Board of Hannover District Government (Hannover, Germany). Animals were housed at the Central Animal Laboratory at Hannover Medical School (Hannover, Germany; specific pathogen free [SPF] conditions, three animals of the same gender per cage, air-conditioned rooms, $24^{\circ} \mathrm{C}, 60^{\circ} \%$ humidity, $12: 12 \mathrm{hr}$ light:dark cycle with artificial lights on at 7:00 a.m., free access to water and Altromin rat chow). The brains were processed as described for the Lewis rat in experiment 1. The entire left brain half and the rest of the right brain half were serially cut into $100 \mu \mathrm{m}$ thick sagittal sections. The boundary between left and right brain half was determined by inspection of the sections, considering landmarks at the middle of the brain such as third ventricle, fourth ventricle, medial parts of the hippocampus etc. Estimates of total number of neurons in specified 
regions of the left hemispheres were carried out using the optical fractionator [5] (applied as recommended by $[23,24]$ ) and a C.A.S.T. - Grid system (Olympus, Denmark), consisting of a modified light microscope (type BX50, Olympus), Olympus UP anApo objectives (40×, oil, $\mathrm{NA}=1.0$ and $100 \times$, oil, $\mathrm{NA}=1.35$ ), a motorized specimen stage for automatic sampling (Maerzhaeuser, Germany), an electronic microcator (Heidenhain, Germany), a CCD colour video cumera (JAI, Denmark), a PC (IBM, England) with framegrabber board (type Screen Machine II, Fast Multimedia, Germany), installed C.A.S.T.-Grid software (Olympus), and a $17^{\prime \prime}$ television screen monitor (IBM). Every third section of the left brain halves were investigated. All hippocampal pyramidal cells (areas CAI-3; distinguished from area CA4 and the subiculum as depicted in [25]) were counted when the top of the nucleus came into focus within optical disectors systematically and randomly spaced throughout this brain region. Therefore, the top of the nuclei (not the nucleoli) served as counting unit. All details of the counting procedure are summarized in Table 1. Optical disectors were also used for counting cerebellar granule and Purkinje cells, also using the top of the nuclei as counting unit. Details of the counting procedures are also given in Table 1. Thickness of the sections was measured according to West et al. [32]. Using the corresponding oil objective and the microcator attached to the stage, the distance between the upper and lower surface of the section, identified by focusing up and down on the section, was measured at the position of every fifth optical disector. The mean tissue thickness was used as the section thickness for the series cut from that individual. Estimated total neuronal numbers were calculated from the number of counted neurons and the sampling probability $[5,23,24]$. Coefficients of error of the estimated total neuronal numbers were calculated as the reciprocal values of the square root of the numbers of counted neurons $[23,24]$. Differences in the mean total neuronal numbers were tested by the Mann-Whitney test. Differences were considered to be significant if $P<0.05$. Calculations were carried out with GraphPad Prism version 3.00 for Windows (GraphPad Software, USA).

Table 1. Details of the stereological analyses of experiment 2.

\begin{tabular}{|c|c|c|c|c|c|c|}
\hline $\begin{array}{l}\text { Group } \\
\text { Gell type }\end{array}$ & \multicolumn{2}{|c|}{$\begin{array}{l}\text { Fischer } 344 \text { Lewis } \\
\text { HPC (area CA1-3) }\end{array}$} & \multicolumn{2}{|c|}{$\begin{array}{l}\text { Fischer } 344 \text { Lewis } \\
\text { Cerebellar granule cells }\end{array}$} & \multicolumn{2}{|c|}{$\begin{array}{l}\text { Fischet } 344 \text { Lewis } \\
\text { Cerebellar Purkinje cells }\end{array}$} \\
\hline ssf & 0.333 & 0.333 & 0.333 & 0.333 & 0.333 & 0.333 \\
\hline Objective & $40 x$ & $40 x$ & $100 x$ & $100 x$ & $40 x$ & $40 x$ \\
\hline frame $\left(\mu m^{2}\right)$ & 3,060 & 3,060 & 197 & 197 & 18,357 & 18,357 \\
\hline height ( $1 \mu \mathrm{m})$ & 7 & 7 & 3 & 3 & 7 & 7 \\
\hline top of frame (km) & -1 & -1 & -1 & -1 & -1 & -1 \\
\hline steps $\left(X_{,} Y_{;} \mu m\right)$ & 225 & 225 & 600 & 600 & 400 & 400 \\
\hline ast & 0.060 & 0.060 & 0.00055 & 0.00055 & 0.115 & 0.115 \\
\hline $\operatorname{tsi}$ & 0.193 & 0.185 & 0.193 & 0.193 & 0.380 & 0.386 \\
\hline$(\text { sst } \times \text { asf } \times \text { isf })^{-1}$ & 258 & 268 & 37,732 & 37,865 & 71 & 68 \\
\hline mean $\mathrm{S} Q$ & 2,345 & 2,240 & 1,292 & 1,369 & 2,087 & 1,960 \\
\hline mean $S$ cs & 700 & 624 & 480 & 478 & 774 & 695 \\
\hline
\end{tabular}

ssf, section sampling fraction; asf, area sampling fraction; $\overline{\text { tsf }}$, average thickness sampling fraction; ( $s \mathrm{sf}^{\mathrm{x}}$ asf $\left.\times \overline{\mathrm{tsf}}\right)^{-1}$, reciprocal value of the sampling probability; mean $S \mathrm{Q}$, mean number of counted neurons; mean $\mathrm{S} \mathrm{CS}_{\text {, }}$ mean number of analyzed counting spaces. 


\section{Results}

Representative photomicrographs of rat tissue used in experiment 1 are shown in Figure 1. Nuclei and nucleoli were observed both in hippocampal area CA3 and cerebellar cortex; even on 3 to $6 \mu \mathrm{m}$ thick sections. The quality of the sections facilitated unambiguous identification of the investigated types of neurons. Representative photomicrographs of human tissue described in experiment 1 are shown in Figure 2. Nuclei and nucleoli were observed both in cerebral and cerebellar cortex, even on $5 \mu \mathrm{m}$ thick sections. The quality of the human tissue sections also facilitated unambiguous identification of the investigated types of neurons. Rarely were nuclei with more than one nucleolus observed.

Estimated total numbers of hippocampal pyramidal cells in areas CAI-3 and cerebellar granule and Purkinje cells of the rats investigated in experiment 2 are shown in Figure $3 \mathrm{~A}$ and Table 2. There were no significant differences between the Fischer 344 and the Lewis rats with respect to estimated mean total numbers of hippocampal pyramidal cells in areas CA1-3 $(P=0.937)$, cerebellar granule cells $(P=0.240)$ and Purkinje cells $(P=0.699)$. Figure $3 \mathrm{~B}$ displays the actual thickness of the analyzed sections for estimating the total numbers of neurons shown in Fig. 3A. No significant differences in mean tissue thickness of the hippocampal areas CA1-3 $(\mathrm{P}=0.180)$ or the cerebellar granule cell layer $(\mathrm{P}=1.000)$ were found between the Fischer 344 and the Lewis rats. However, for the cerebellar Purkinje cell layer, a very small but significant difference was found between the Fischer 344 and the Lewis rats (Mann Whitney test; $P=0.002$ ). Mean predicted coefficients of error of the estimated total neuronal numbers varied between 0.021 and 0.028 . Mean thickness shrinkage factors were 0.37 for hippocampal areas CA1-3,0.156 for cerebellar granule cell layer and 0.185 for cerebellar Purkinje cell layer. The estimated mean total neuronal numbers presented here are consistent with previous estimates shown in Table 3 and 4.

Table 2. Results of the stereological analysis of Section 2.2

\section{Group Cell type}

\# of cells - mean

\# of cells - SEM

mean predicted

coefficient of error

\section{Fischer 344 Lewis HPC (area CA1-3)}

604,457
28,442
0.021

603,439

41,421

0.021

\section{Fischer 344 Lewis Cerebellar granule cells}

$\begin{array}{cc}48,738,689 & 51,848,939 \\ 3,874,494 & 2,794,518 \\ 0.028 & 0.027\end{array}$

$48,738,689$

0.028

\section{Fischer 344 Lewis Purkinje cells}

$\begin{array}{rc}144,647 & 132,742 \\ 11,333 & 7,128 \\ 0.022 & 0.023\end{array}$




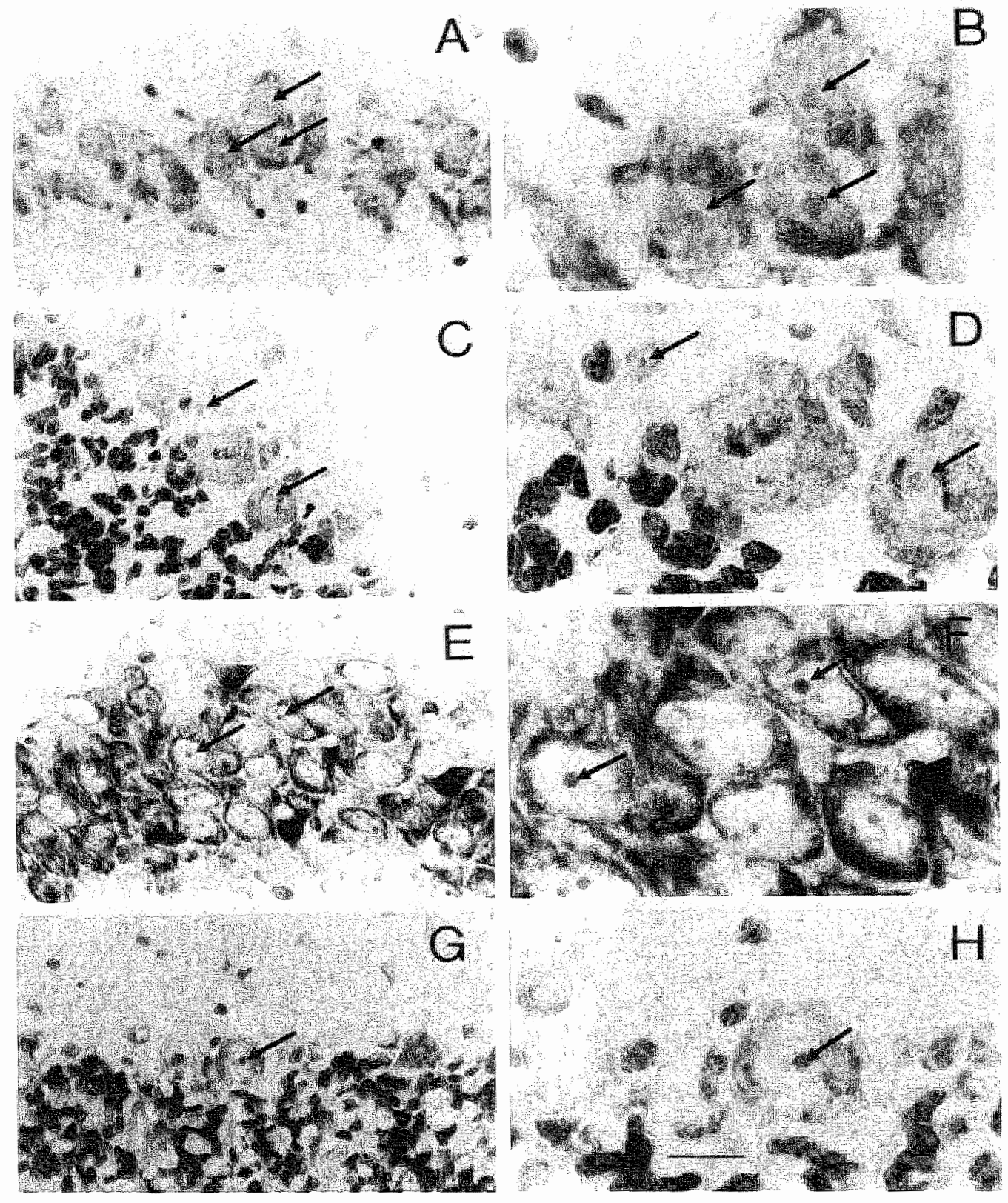

Figure 1. Photomicrographs of cryostat sections of rat brains, demonstrating the presence of neuronal nucleoli in 3 to $6 \mu \mathrm{m}$ thick sections. (A, B) Brain of a 7 month old Lewis rat, snap-frozen, $3 \mu \mathrm{m}$ thick sagittal section stained with cresylviolet, hippocampal area CA3. $(C, D)$ Same section, cerebellar granule and Purkinje cell layers. (E, F) Brain of a one month old Wistar rat, perfusion-fixed with formalin, $6 \mu \mathrm{m}$ thick sagittal section stained with cresylviolet, hippocampal area CA3. $(G, H)$ Same section, cerebellar granule and Purkinje cell layers. Nucleoli are marked by arrows. Bar, $20 \mu \mathrm{m}$. 

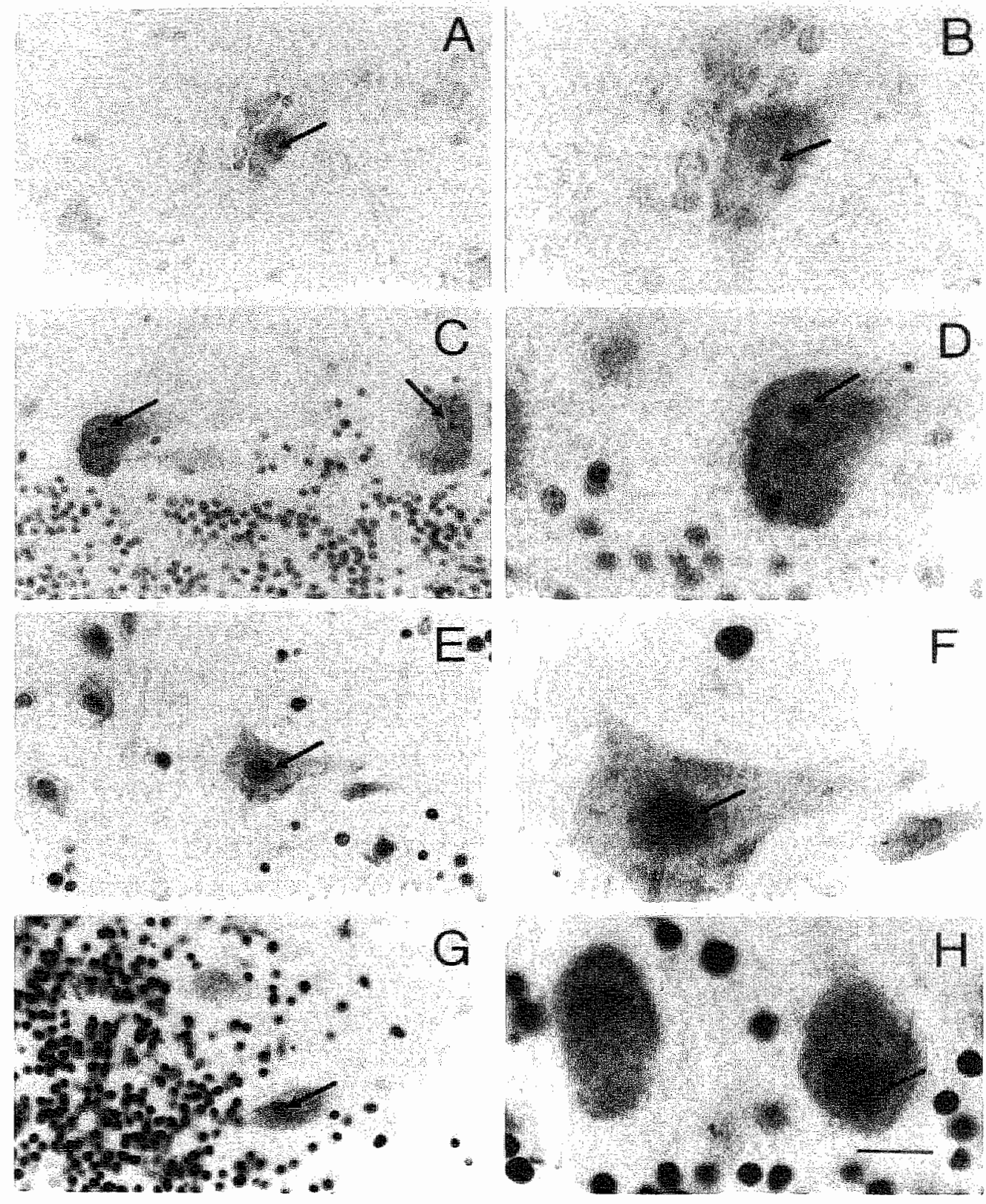

Figure 2. Photomicrographs of cryostat sections of a human brain (male, 29 years old, immersion-fixed with formalin for approximately 24 months), demonstrating the presence of neuronal nucleoli in $5 \mu \mathrm{m}$ thick sections. $(A, B)$ Section stained with cresylviolet, cerebral cortex, layer $\mathrm{V}$. (C, D) Section stained with cresylviolet, cerebellar granulle and Purkinje cell layers. $(E, F)$ Section stained with gallocyanin, cerebral cortex, layer $V(G, H)$ Section stained with gallocyanin, cerebellar granule and Purkinje cell layers. Nucleoli are marked by arrows. Bar, 20um. 
Table 3. Compilation of estimated mean total numbers of HPC

\begin{tabular}{|c|c|c|c|c|}
\hline Authors & Strain & Sex & Age [months] & if of $\mathrm{HPC}(\mathrm{CA} 1-3)$ \\
\hline [this study] & Fischer 344 & Male & 7 & 604,457 \\
\hline [this study] & Lewis & Male & 7 & 603,439 \\
\hline Rapp and Gallagher (1996) & Long-Evans & Male & 6 & $w 605,000$ \\
\hline Rapp and Gallagher (1996) & Long-Ewans & Male & $27-28$ & 625,000 \\
\hline West et al. (1991) & Wistar & $?$ & 1 & 632,000 \\
\hline Rasmussen et al. (1996) & Wistar & Male & 2 & 930,000 \\
\hline Sousa et al. (1999) & Wistar & Male & 7 & $* 660,000$ \\
\hline Lukoyanov et al. (2000) & Wistar & Male & 9 & 650,000 \\
\hline Lukoyanov et al. (1999) & Wistar & Male & 14 & 687,000 \\
\hline Rasmussien et al. (1996) & Wistar & Male & 24 & $1,020,000$ \\
\hline
\end{tabular}

Data are given as unilateral values. $t$, data obtained using methacrylate sections and the optical disector.
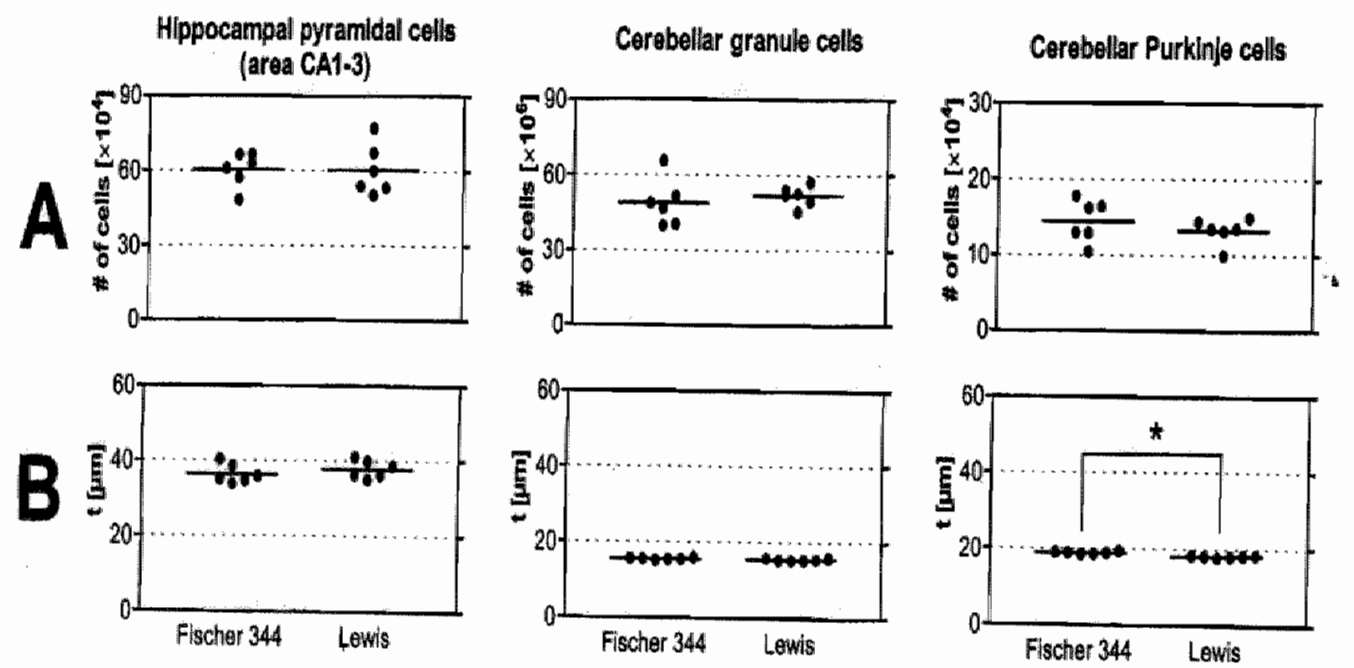

\section{Group}

Figure 3. (A) Estimated totall numbers of hippocampal pyramidal cells in areas CA $1-3$ and cerebellar granule and Purkinje cells of 7 -month-old male Fischer 344 rats and 7 -monthold male Lewis rats. There are no significant differences between the Fischer 344 and the neurons shown and in the sections for estimating the totambers of ween the Fis in (A). Note that there are only small differences in section thickness bet hippocampal areas 
Table 4. Compilation of estimated mean total numbers of cerebellar granule cells (CGC) and Purkinje cells (CPC) in the rat brain.

\begin{tabular}{|c|c|c|c|c|c|}
\hline Authors & Strain & Sex & Age [months] & \# of GC & A of PC \\
\hline [this study] & Fischer 344 & Male & 7 & $97,477,378$ & 289,294 \\
\hline [this study] & Lewis & Male & 7 & $103,697,879$ & 265,484 \\
\hline Bedi et al. (1992) & Lister & $?$ & 1 & & 204,300 \\
\hline Bedi et al. (1992) & Lister & $?$ & 2 & & 188,200 \\
\hline Bedi et al. (1992) & Lister & ? & 7 & & 253,800 \\
\hline $\begin{array}{l}\text { Armstrong and Schild } \\
(1970)\end{array}$ & Wistar & Male & 2.5 & & 326,000 \\
\hline $\begin{array}{l}\text { Harvey and Napper } \\
\text { (1988) }\end{array}$ & Wistar & ? & [adult] & $92,000,000$ & 338,000 \\
\hline Hillman and Chen (1981) & Wistar & $?$ & [adult] & $\approx 90,000,000$ & 278,000 \\
\hline Larsen et al. (1994) & Wistar & Male & 4 & $3300,000,000$ & $\approx 780,000$ \\
\hline Korbo et al. (1993) & Wistar & Male & 7 & $265,000,000$ & 610,000 \\
\hline
\end{tabular}

Results presented in this study (Table 2) were doubled to make comparisons with data in the literature easier. $\neq$, data obtained using paraffin sections and a modification of the fractionator; $t$, data obtained using methacrylate sections and the optical disector.

\section{Discussion}

Recently, Andersen and Gundersen (1999) reported a loss of all nucleoli of neurons in the upper $5 \mu \mathrm{m}$ of $100 \mu \mathrm{m}$ thick vibratome sections in the cerebellar nucleus dentatus of a human brain, as well as a loss of almost all nucleoli in the lowest $10 \mu \mathrm{m}$ of these sections [1]. The authors suggested that in this unembedded tissue, cells hit by the knife may have lost their nuclei across the unprotected section surfaces. The results of experiment 1 presented here clearly show that this is not a general feature of sections of unembedded tissue. Even in $3 \mu \mathrm{m}$ thick serial sections of snap-frozen rat brains (i.e., of tissue without any fixation before sectioning), we were able to detect many nuclei and nucleoli of hippocampal pyramidal cells and cerebellar Purkinje cells (Fig, 1A-D). The same was found for $6 \mu \mathrm{m}$ thick sections of rat brains following perfusion-fixation with formalin (Fig. $(\mathrm{E}-\mathrm{H}$ ) and for $5 \mu \mathrm{m}$ thick sections of human brain following immersion-fixation with formalin (Fig. 2). The differences between these results and the findings of Andersen and Gundersen (1999) are likely due to differences in the manner of preparing brain sections [1]. Protocols for obtaining adequate cryostat sections of the rodent brain for optical disector analysis are given above. Corresponding protocols for the human brain may be found in this issue of the Journal of Chemical Neuroanatomy $[9,20]$.

Based on their finding of a lack of nucleoli in the upper $5 \mu \mathrm{m}$ and the lower $10 \mu \mathrm{m}$ of their 100 $\mu \mathrm{m}$ thick vibratome sections, Andersen and Gundersen (1999) concluded that one must respect rather large guard zones close to the upper and lower surfaces of thick sections of unembedded tissue if using the optical disector for counting neurons [1]. However, the results of experiment 
2 show that the use of large guard zones are not necessary in order to reliably estimate total neuronal numbers using the optical disector in all unembedded tissue. When placing the top of the counting frames only $1 \mu \mathrm{m}$ below the upper surface of the sections of the snap-frozen rat brains, we obtained estimated total numbers of hippocampal pyramidal cells and cerebellar granule and Purkinje cells which were similar to data reported in the literature (Table 3 and 4 ; data obtained using methacrylate sections and the optical disector are marked). The reason why Korbo et al., Larsen et al. and Rasmussen et al. have obtained higher estimates than reported earlier in the literature has not been discussed by these authors and remains therefore unknown $[12,14,22]$.

The reason for the greater thickness shrinkage found here for the cerebellum than for the hippocampus is unknown (mean thickness shrinkage factor of 0.156 for cerebellar granule cell layer and 0.185 for cerebellar Purkinje cell layer, and 0.37 for hippocampal areas CAI3). Interestingly, this difference between cerebellum and hippocampus was not observed for $100 \mu \mathrm{m}$ thick sagittal cryostat sections of mouse brains perfusion-fixed with formalin (mean thickness shrinkage factor of 0.32 for cerebellar granule and Purkinje cell layer, and 0.34 for hippocampal areas CA1-3; 25,26$]$; all mentioned hippocampal data are similar to thickness shrinkage factors which can be calculated from data previously reported for frozen sections of the rat striatum $[15,31])$. This points to the need for determining the actual section thickness for any analyzed area rather than to use correction factors for tissue shrinkage reported in the literature. Note that the studies by Schmitz er al. provide protocols for obtaining reliable estimates of total neuronal numbers using cryostat sections of cryoprotected tissue of perfusion-fixed animals, which may also be used for immunohistochemical or in situ hybridization analysis $[25,26]$.

To summarize, cryostat sections of snap-frozen nervous tissue may successfully be used for estimating total neuronal numbers using optical disectors. Contrary to methacrylate sections, the use of cryostat sections of snap-frozen tissue opens the possibility for combining estimates of total neuronal numbers with any kind of histological, cellular, or molecular analysis of adjacent sections of the same brain. It can be expected that such combined investigations will be one of the most dynamic aspects of applied neurostereology in the near future.

\section{Acknowledgements}

The authors wish to thank Miss Helga Helten for excellent technical assistance. This study was supported by grants from the START-program of the Faculty of Medicine at the RWTH University of Aachen, Germany (CS), from the Volkswagen Foundation (SvH; $1 / 75$ 169) and from EC (HK; NRPB Association Contract No. F14P-CT95-0008). 


\section{References}

[1] Andersen, B.B., Gundersen, H.J.G., 1999. Pronounced loss of cell nuclei and anisotropic deformation of thick sections. J. Microsc. 196, 69-73.

[2] Armstrong, D.M., Schild, R.F., 1970. A quantitative study of the Purkinje cells in the cerebellum of the albino rat. J. Comp. Neurol. $139_{n} 449-456$.

[3] Biedi, K.S., Campbell, L.F., Mayhew. T.M., 1992. A fractionator study of the effects of undernutrition during early life on rat Purkinje cell numbers (with a caveat on the use of nucleoli as counting units) J. Anat. 181, 199-208.

[4] Fischer, C.P., Gundersen ${ }_{\mathrm{s}}$ H.J.G., Pakkenberg, B., 1999. Preferential loss of large neocortical neurons during HIV infection: a study of the size distribution of neocortical neurons in the human brain. Brain Res. 828, 119-126.

[5] Gundersen, H.J.G., 1986. Stereology of arbitrary particles. J. Microsc. 143, 3-45.

[6] Harvey, R.J., Napper, R.M.A., 1988. Quantitative study of granule and Purkinje cells in the cerebellar cortex of the rat. J. Comp. Neurol. 274, 151-157.

[7] Hatton, W.J., Von Bartheld, C.S. 1999. Analysis of cell death in the trochlear nucleus of the chick embryo: calibration of the optical disector counting method reveals systematic bias. J. Comp. Neurol. 409, 169-186.

[8] Heinsen, H., Rüb, U., Bauer, M., Ulmar, G., Bethke, B., Schüler, M., Böcker, F., Eisenmenger, W., Götz, M., Korr, H., Schmitz, C., 1999. Nerve cell loss in the thalamic mediodorsal nucleus in Huntington's disease. Acta Neuropathol. 97, 613-622.

[9] Heinsen, H., Arzberger, T., Schmitz, C., 2000. Celloidin embedding of human brains - a new, simple and reliable method for producing serial sections of high section thickness. $J$. Chem. Neuroanat., in press.

[10] Hillman, D.E., Chen, $S_{*}, 1981$. Vulnerability of cerebellar development in malnutrition - I. Quantitation of layer volume and neuron numbers. Neurosci. 6,1249-1262.

[11] Jinno, S., Aika, Y., Fukuda, T., Kosaka, T., 1998. Quantitative analysis of GABAergic neurons in the mouse hippocampus, with optical disector using confocal laser scanning micrascopy. Brain Res. 814, 55-70.

[12] Korbo, L., Andersen, B.B., Ladefoged, O., Møller, A., 1993. Total numbers of various cell types in rat cerebellar cortex estimated using an unbiased stereological method. Brain Res. 609, 262-268.

[13] Korbo, L., Ladefoged, O., Lam, H.R., Ostergaard, G., West, M.J., Arlien-Søborg, P., 1996. Neuronal loss in hippocampus in rats exposed to toluene. Neurotoxicol. 17, 359.366.

[14] Larsen, J.O., Tandrup. T., Braendgaard, H. "1994. The volume of Purkinje cells decreases in the cerebellum of acrylamide - intoxicated rats, but no cells are lost. Acta Neuropathol. 88 , $307-312$.

[15] Long, J.M., Kalehua, A.N., Muth, N.J., Hengemihle, J.M., Jucker, M., Calhoun, M.E., Ingram, D.K., Mouton, P.R., 1998. Stereological estimation of total microglia number in mouse hippocampus. J. Neurosci. Meth. 84, 101-108.

[16] Lukoyanov, N.V., Madeira, M.D. Paula-Barbosa, M.M., 1999. Behavioral and neuroanatomical consequences of chronic ethanol intake and withdrawal. Physiol. Behav. 66, 337-346.

[17] Lukoyanov, N.V., Brandlao, F."Cadete-Leite, A., Madeira, M.D., Paula-Barbosa, M.M. 2000. Synaptic reorganization in the hippocampal formation of alcohol-fed rats may compensate for functional deficits related to neuronal loss. Alcohol 20,139-148.

[18] Meaney, M.J., Diorio, J., Francis, D. Weaver, S., Yau, J., Chapman, K., Seckl, J.R., 2000. Postnatal handling increases the expression of cAMP-inducible transcription factors in the rat hippocampus: the effects of thyroid hormones and serotonin. J. Neurosci. 20, 39263935. 
[19] Messina, A., Sangster, C.L.C., Morrison, W.A., Galea, M.P., 2000. Requirements for obtaining unbiased estimates of neuronal numbers in frozen sections. J Neurosci. Meth. $97,133-137$.

[20] Perl, D.P. Good, P.F., Morrison, J.H., Erwin, J.M., Hof, P.R., 2000. Practical approaches to stereology in the setting of aging-and disease-related brain banks. J. Chem. Neuroanat., in press.

[21] Rapp $P_{1,}$ Gallagher, $M_{2}, 1996$. Preserved neuron number in the hippocampus of aged rats with spatial learning deficits. Proc. Natl. Acad. Sci. USA 93, 9926-9930.

[22] Rasmussen, T., Schliemann, T., Sørensen, J.C., Zimmer, J., West, M.J., 1996. Memory impaired aged rats: no loss of principal and subicular neurons. Neurobiol. Aging 17, 143147.

[23] Schmitz, C., 1998. Variation of fractionator estimates and its prediction. Anat. Embryol. 198, 371-397.

[24] Schmitz ${ }_{n}$ C., Hof , P.R. 2000. Recommendations for straightforward and rigorous methods of counting neurons based on a computer simulation approach. $J$ Chem Neuroanat, in press.

[25] Schmitz, C., Bültmann, E., Gube, M., Korr, H., 1999. Neuron loss in the mouse hippocampus following prenatal injection of tritiated thymidine or saline. Int. J. Dev. Neurosci. 17, 185-190.

[26] Schmitz, C., Otto, UU., Korr, H., 2000. More cerebellar granule cells following a prenatal low dose X-irradiation. Brain Res., in press.

[27] Sousa, N., Paula-Barbosa, M.M., Almeida, O.F., 1999. Ligand and subfiell specificity of corticoid-induced neuronal loss in the rat hippocampal formation. Neurosci. 89, 1079-1087.

[28] Van Oers, H.J, De Kloet, E.R., Whelan, T., Levine, S. (1998) Maternal deprivation effect on the infant's neural stress markers is reversed by tactile stimulation and feeding but not by suppressing corticosterane. J. Neurosci. 18, 10171-10179.

[29] Von Bartheld, C.S., 1999. Systematic bias in an "unbiased" neuronal counting technique. Anat. Rec. 257, 119-120.

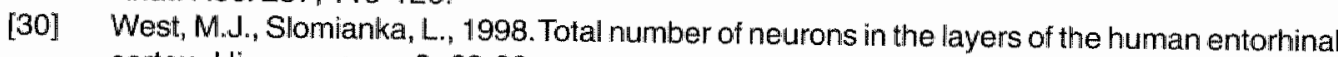
cortex. Hippocanipus 8, 69-82.

[31] West, M.J., Slomianka, L., Gundersen, H.J.G., 1991. Unbiased stereological estimation of the total number of neurons in the subdivisions of the rat hippocampus using the optical fractionator. Anat. Rec. 231, 482-497.

[32] West, M.J., Ostergaard, K., Andreassen, O.A., Finsen, B., 1996. Estimation of the number of somatostatin neurons in the striatum. An in situ hybridization study using the optica: fractionator method. J. Comp. Neurol. 370, 11-22. 


\section{Perinatal asphyxia induced neuronal loss by apoptosis in the neonatal rat striatum}

A combined TUNEL and stereological study

W.D.J Van de Berg, C. Schmitz, H.W.M. Steinbusch, C.E. Blanco

Experimental Neurology 2002;174:29-36 


\section{Abstract}

Perinatal asphyxia can lead to cell damage in various regions of the brain, such as the neostriatum. In this study, we investigated the mechanism of cell death that leads to neuron loss in the neostriatum of rat pups. Asphyxia was induced by immersing fetus-containing uterus horns in a water bath at $37^{\circ} \mathrm{C}$ for $20 \mathrm{~min}$. This led to an increase in mortality rate $( \pm 40 \%)$ compared to control pups $(0 \%)$. TUNEL-positive cell profiles were visible in all groups at postnatal day $(\mathrm{P}) 2, \mathrm{P} 8$ and $\mathrm{P} 15$, peaking at $\mathrm{P} 8$. A significant increase of $40 \%$ at $\mathrm{P} 8$ and $45 \%$ at $\mathrm{P} 15$ in the number of TUNEL-positive cell profiles was observed in asphyctic rats compared to control rats. Nuclear condensation and fragmentation was visible with the DNA stain Hoechst 33324. Furthermore, laser scanning confocal microscopy showed multiple DNA fragments in TUNEL-positive cell profiles. We found a decrease of $16 \%$ in the total number of striatal neurons in the asphyctic pups compared to the control pups at 21 days post-asphyxia using stereology. These data show that asphyxia causes exaggerated apoptotic cell death during the first week of life and as a consequence a small amount of neuron loss in the neostriatum. 


\section{Introduction}

Complications during pregnancy or birth, such as asphyxia, can have a long-term effect on the developing brain. These insults cannot only lead to mental retardation and/or motor disabilities, but also to mortality $[5,55,66]$. Many studies have tried to unveil the mechanism for the pathologic processes leading to cerebral dysfunction $[3,13]$. Cellular mechanisms, such as the presence of antioxidant defense systems, the development and modulation of excitatory neurotransmitter receptors and $\mathrm{Ca}^{2+}$-dependent mechanisms, have been shown to be important determinants of the susceptibility of the developing brain to asphyxia $[44,54,62]$. Furthermore, certain regions in the brain, such as the neostriatum, thalamus and hippocampus seem to be more vulnerable to a hypoxic insult $[1,14,40,47]$.

Cell death in the immature brain is mostly studied using the TUNEL (terminal deoxynucleotidyl transferase (TdT) -mediated dUTP nick-end labeling) assay, DNA markers, histological staining methods or electron microscopy (EM) $[12,27,50]$. In the TUNEL assay, TdT binds to exposed 3'-OH ends of DNA strand breaks, generated principally by apoptosis signals [17]. Apoptosis and necrosis are distinguished on the basis of different morphological and biochemical characteristics, such as organized chromatin condensation and preservation of plasma membrane integrity in apoptosis contrast with cytolysis and tissue inflammation in necrosis $[29,30,68]$. Several observations have shown that apoptosis plays an important role in the central and peripheral nervous system, where it regulates developmental, physiological and pathological cell death $[53,67]$.

Several animal models are used to study shorl-term and long-term effects of perinatal asphyxia $[9,28]$. In our study, we use an animal model that induces global asphyxia during delivery, leading to chronic neurotransmitter alterations such as an increase in striatal dopamine levels and a decrease in gamma-amino butyric acid (GABA) and aspartate levels $[10,15,34$, $35,36]$. Furthermore, an increase in the number of TUNEL-stained cells has been shown in this model in the frontal cortex, striatum and cerebellum at postnatal day P8 [14]. However, none of these studies showed neuron loss due to perinatal asphyxia in the aforementioned brain areas.

Since the striatum is one of the most vulnerable regions of the brain for perinatal asphyxia, we determined neuron damage and neuron loss in this area, as a consequence of perinatal asphyxia, using a modern quantitative neuro-anatomical approach. We applied TUNEL to label double-stranded DNA breaks. In addition, we analyzed brain sections for morphological changes, such as nuclear condensation and fragmentation with Hoechst and HematoxylinEosin staining, in conjunction with confocal microscopy. Finally, we investigated neuron loss by using state-of-the-art stereology. 


\section{Materials and methods}

\section{Animals}

Forty-two full-term pregnant $W$ istar rats and their male pups $(n=72)$ were used. Dams were purchased from Charles River-Broekmans (Someren. The Netherlands). They were housed in the Animal Care Department of the University of Maastricht under standard conditions (12:12 h light:dark cycles, $20^{\circ} \mathrm{C}$, with free access to standard laboratory chow and water). The ethical board of University of Maastricht approved animal care and procedures.

\section{Induction of perinatal asphyxia}

Asphyxia was induced in rat fetuses at P0 by placing the uteri and its contents in a water bath for 20 minutes, as described previously in Van de Berg et al. [65]. Briefly, dams were decapitated immediately after one or two pups were delivered vaginally (CVD) on day 21 or 22 of pregnancy. Afterwards, dams were rapidly hysterectomized and the uterus horns containing the remaining fetuses were detached and one hom was placed in a water bath at $37^{\circ} \mathrm{C}$ for $20 \mathrm{~min}$ (severe perinatal asphyxia; SPA). The other horn was rapidly opened and pups were removed and placed in an incubator at $37^{\circ} \mathrm{C}$ (time of procedure $<30$ sec; control cesarean delivery; CCD). After $20 \mathrm{~min}$ in the water bath (SPA), pups were removed from the uterus horn and stimulated to breath by cleaning skin and by gently padding them on the chest. The maximal duration of the resuscitation was 5 minutes. If a pup was not able to gasp, it was excluded from the study. The procedures for cleaning and resuscitation were performed in an incubator at $37^{\circ} \mathrm{C}$. The pups were left to recover for $60 \mathrm{~min}$ in the incubator, marked and then placed with a surrogate mother. Each surrogate mother received 10 pups. The percentage of mortality in the SPA group was $\pm 40 \%$. All pups belonging to the control groups (i.e., CVD and CCD) were gasping immediately after birth and all survived.

\section{General technical procedures}

At P2, P8 and P15, the pups were anaesthetized with sodium pentobarbital $(60 \mathrm{mg} / \mathrm{kg}$; i.p.; Nembutal) and perfused through the heart $(50 \mathrm{mmHg})$, first with tyrode, followed by the fixative containing $4 \%$ paraformaldehyde and $2 \%$ picric acid in $0.1 \mathrm{M}$ phosphate buffer ( $\mathrm{PB}$, pH 7.6, room temperature; RT). Following perfusion, the brain was removed from the skull and postfixed for 24 hours at $4{ }^{\circ} \mathrm{C}$ in $4 \%$ paraformaldehyde in $0.1 \mathrm{M}$ PB for TUNEL and Hematoxylin-Eosin (HE) staining, or for 72 hours at $4^{\circ} \mathrm{C}$ in the same solution for stereology. Brain tissue was cryoprotected either by immersion in $15 \%$ sucrose/0.1 M TBS overnight at $4 \mathrm{C}$ for TUNEL and HE staining, or by immersion in $30 \%$ sucrose $0.1 \mathrm{M}$ TBS for 48 hours at $4^{\circ} \mathrm{C}$ for stereological analysis. Afterwards, the brain was quickly frozen by placement in isopentan for $25 \mathrm{sec}$, cooled with liquid nitrogen and stored at $-80^{\circ} \mathrm{C}$ until further processing.

\section{TUNEL labeling of dying cells}

The brain was entirely cut to serial, $16 \mu \mathrm{m}$ thick, coronal sections on a cryostat. Every eight section was used for TUNEL staining by using the method developed by Gavrieli et al. [17],
slightly modified to accommodate frozen sections of pup tissue. Sections were permeabilized 
for 15 min using methacarn (methanol: chloroform: acetic acid $=66: 33: 1 ; \mathrm{v} / \mathrm{v} / \mathrm{v}$ ), rinsed in TBS ( $2 \times 10 \mathrm{~min})$ and incubated in terminal transferase (TdT) buffer ( $1 \mathrm{M}$ potassium cacodylate, $125 \mathrm{mM}$ Tris, Bovine serum Albumin (BSA) $1.25 \mathrm{mg} / \mathrm{ml}$ and $25 \mathrm{mM}$ cobalt chloride ( $\mathrm{pH}$ 6.6, $\left.25^{\circ} \mathrm{C}\right), \mathrm{TdT}(0.3 \mathrm{e} . \mathrm{u} / \mathrm{\mu l})$ and biotinylated $\mathrm{dUTP}(0.04 \mathrm{mM})$ (Boehringer Mannheim, Indianapolis, $\mathrm{NN}$, USA) in a humid chamber at $37^{\circ} \mathrm{C}$ for $2 \mathrm{hr}$. During incubation, sections were covered with $20 \mu \mathrm{l}$ of the incubation solution and coverslipped with parafilm. The reaction was stopped by placing the slides in $2 \times$ SSC buffer ( $300 \mathrm{mM}$ sodium chloride, $30 \mathrm{mM}$ sodium nitrate; $\mathrm{pH} 7.6$ ) for $20 \mathrm{~min}$ at RT, followed by TBS for $10 \mathrm{~min}$. Subsequently, streptavidin-Cy 3 (1:2000 dilution; for $60 \mathrm{~min}$ at RT) was used to visualize nuclei labeled with biotinylated dUTP. After washing in TBS, sections were counterstained with Hoechst 33324 (1:1000 dilution; $60 \mathrm{~min}$ at RT; Sigma), rinsed again in TBS and mounted with TBS:glycerol ( $1: 3)$.

As a negative control, sections were subjected to the above procedure, with either TdT or biotin-16-dUTP omitted. As a positive control, sections were pretreated with $\| \mu \mathrm{g} / \mathrm{ml}$ DNAse (Boehringer Mannheim, Indianapolis, IN, USA) for $60 \mathrm{~min}$ at $37^{\circ} \mathrm{C}$ prior to the incubation with the TdT reaction mixture. All cell nuclei were positively stained after DNAse treatment.

\section{Quantitative and confocal analysis of TUNEL-stained cells}

TUNEL-stained cells were counted in tissue of 6 rats of each group (SPA, CCD and CVD) at each of the following time points: P2, P8 and P15. To do so, all stained sections were analyzed which contained the striatum, starting at Bregma $2.20 \mathrm{~mm}$. Sections were examined at a magnification of $\times 400$ with an Olympus $\mathrm{AX}-70$ microscope, using epifluorescent illumination (using the counting method described by Capurso et al. [7]). All TUNEL-stained cell profiles in the striatum were counted regardless morphology, using the corpus callosum and lateral ventricle as landmarks. The total number of positive cells in each rat was estimated by multiplying the sum of TUNEL-stained cell profiles in all sections by the sampling interval (i.e., equal to eight). Furthermore, for every TUNEL-stained cell profille it was checked whether the respective cell was highly pyknotic and whether the nucleus was fragmented, with the Hoechst 33324 staining.

Laser scanning confocal microscopy was performed to visualize and localize fragmented DNA strands with a Leica TCS NT system (Leica Microsystems, Heidelberg, Germany). The argon-krypton laser was used to excite the fluorochromes at $568 \mathrm{~nm}(\mathrm{Cy} 3)$. The fluorescence emission was separated with a $580 \mathrm{~nm}$ dichroic beam splitter first. Thereafter, the CY3 fluorescence was selected by a $590 \mathrm{~nm}$ long pass filter. This way cross-over fluorescence was negligible. Distribution and amplitude of fluorescent structures were characterized on dynamic sequences of images ( 32 images).

\section{Analysis of HE stained sections}

For each TUNEL-stained section, the adjacent section was stained with Hematoxylin-Eosin (HE). Representative TUNEL-stained cell profiles in the TUNEL-stained sections were identified in the adjacent HE sections and checked for nuclear and cytoplasmic morphology. 


\section{Stereological analysis}

For stereological analysis 18 rat pups ( $\mathrm{n}=6$ for each group) were perfused at $\mathrm{P} 21$. At this time-point, there were no TUNEL-positive cells visible. Brain tissue of the pups was entirely cut to serial, $100 \mu \mathrm{m}$ thick coronal sections. Due to inadequate dissecting and staining results, the brain from one SPA pup and one CCD pup was excluded from the study. Every second section was used for stereological analysis (starting randomly with either the first or the second section), yielding 8-10 sections per animal. Sections were stained with cresyl violet $(0.01 \%, 35 \mathrm{~min})$. Estimates of total striatal neuron numbers were conducted using the optical fractionator $[21,56]$.

All striatal cells were counted at a final magnification of $x 3,600$, which came into focus within approximately 450 systematically randomly spaced optical disectors through the left striatum (distance between disectors in mutually orthogonal directions $\mathrm{x}$ and $\mathrm{y}$ on the sections: $250 \mu \mathrm{m}$ ). Disectors were $10 \mu \mathrm{m}$ high in an average actual section thickness of approximately $31 \mu \mathrm{m}$, measured with an electronic microcator attached to the microscope. The frame area was $246 \mu \mathrm{m}^{2}$. All quantification was done with the C.A.S.T.-Grid software (Olympus, Denmark). Estimated total numbers of neurons were calculated from the number of counted neurons (on average approximately 600 striatal neurons per animal) and the sampling probability $[21,56]$. Sampling was optimized for prevention of type II error probability due to stereological sampling $[23,57,58]$. The precision of the estimated total numbers of neurons was predicted as recently explained in detail $[19,56]$.

\section{Statistical analysis}

The Kruskall-Wallis test was applied to test the effect of perinatal asphyxia regarding the number of TUNEL-stained cell profiles and the total number of striatal neurons. MannWhitney-U tests were applied to test whether or not the means were significantly different from each other (pair wise comparisons). Differences were considered to be significant if $\mathrm{P}<0.05$. The multistage Bonferroni correction was applied to reveal unacceptably high levels of type I error [59]. All calculations were done using the Statistical Package for the Social Sciences (SPSS Inc., Vermont, USA).

\section{Results}

\section{Morphology}

High-resolution light microscopic analysis of asphyctic rat brains and control rat brains resulted in a similar range of neurodegenerative profiles. No distinction based on morphological features was possible between developmental cell death and cell death induced by perinatal asphyxia. Morphological features of apoptosis, such as condensation, shrinkage and fragmentation were seen in sections of both asphyctic and controls. No vacuolated type or necrotic type of morphology was detectable (Figure 1). Degenerating neurons were darkly 
stained with $\mathrm{HE}$ and their size and shape was irregular. Nuclear fragmentation was visible with HE, TUNEL and with Hoechst 33324 staining within $85 \%$ of the stained cells. Fragmented cells were often more eosinophilic than other cells. Furthermore, confocal analysis showed internucleosomal DNA fragmentation in all TUNEL-positive cells (see Figure 1). The TUNELstained cell profiles were mainly distributed in the vicinity of the lateral ventricle and in the dorsal part of the neostriatum in both asphyctic, as control animals. Within the ventral part of the neostriatum only a few stained cells were visible.
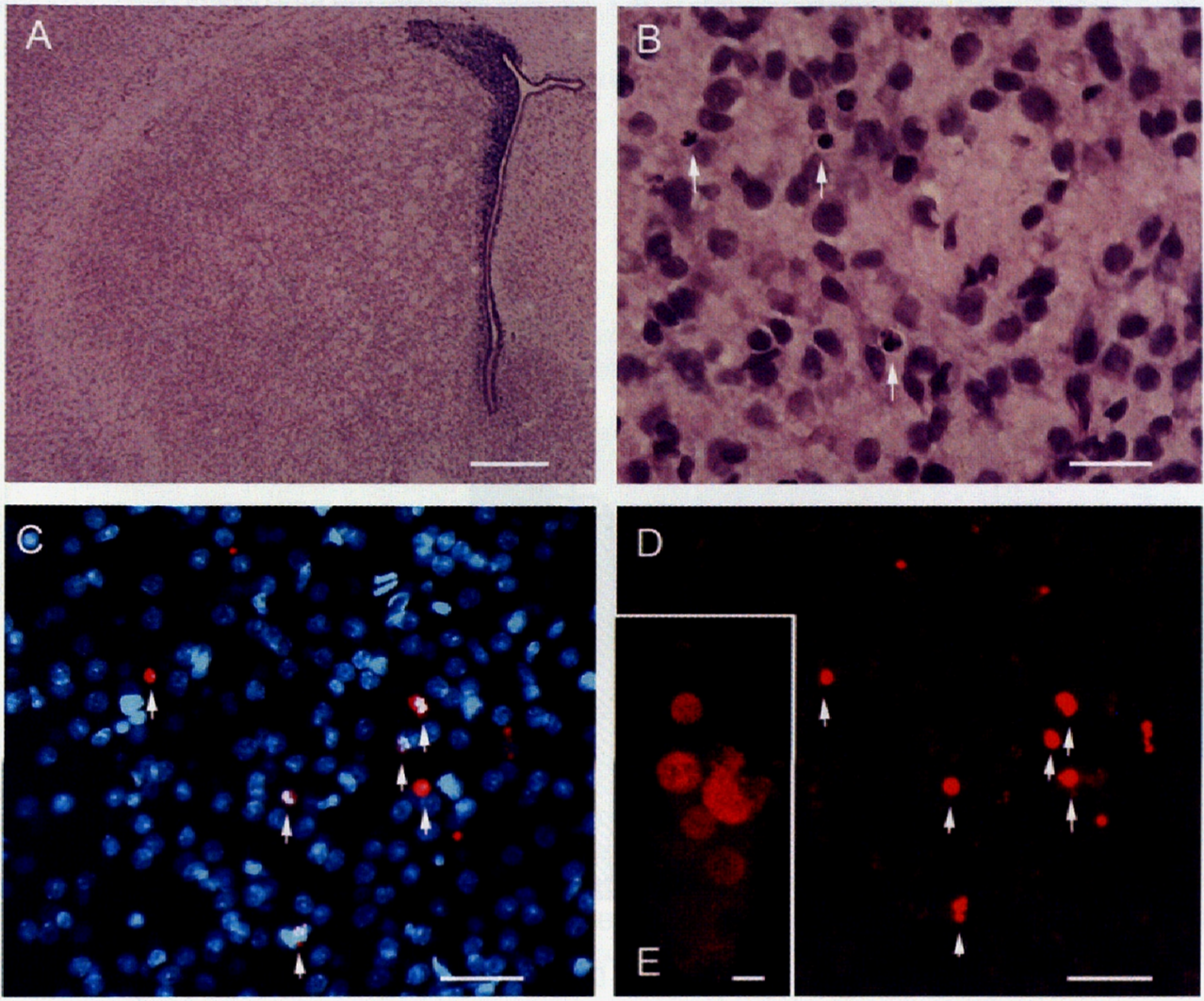

Figure 1. Apoptotic cell profiles in the rat striatum 8 days following perinatal asphyxia as depicted with HE (A, B), Hoechst (C) and TUNEL (D). A. Overview of a section stained with $\mathrm{HE}$. The corpus callosum and lateral ventricle were used as borders of the striatum. $\mathrm{B}$. Higher magnification of the framed area in A. Fragmented nuclei (note single arrows) and condensated nuclei (double arrows) are visible. C, D. Sections stained with TUNEL (D) and counterstained with Hoechst (C), showing fragmented nuclei (single arrows and condensed nuclei (double arrows). Double-staining shows clear fragmentation in TUNELstained cell profiles (compare C and D). E. Confocal image of TUNEL-stained cell profile in the striatum following perinatal asphyxia. Single fragments are visible. Scale bars: $A: 1$ $\mathrm{mm}$; B-D: $40 \mathrm{~mm}$; E: $5 \mathrm{~mm}$. 


\section{Time course of TUNEL-stained cell profiles during development and after asphyxia}

Figure 2 summarizes the results of the quantitative analysis of TUNEL-stained cell profiles in the striatum. TUNEL-stained cell profiles were visible at all time points in both asphyctic and control animals, reaching a peak at 8 days. At P2, there were approximately $2800( \pm$ TUNEL-positive cell profiles visible in the striatum of asphyctic animals and 2900 TUNELpositive cell profiles in the striatum of control animals. At P8, there were approximately 9600 TUNEL-positive cell profiles visible in the striatum of asphyctic animals and 5800 TUNEL-positive cell profiles in the striatum of control animals. At P15, approximately 2000 TUNEL-positive cell profiles were visible in the striatum of asphyctic and 900 TUNEL-positive cell profiles in the striatum of control animals. The difference between the SPA and CVD is $40 \%(\mathrm{P}=0.02)$ at $\mathrm{P} 8$ and $45 \%(\mathrm{P}=0.02)$ at $\mathrm{P} 15$.

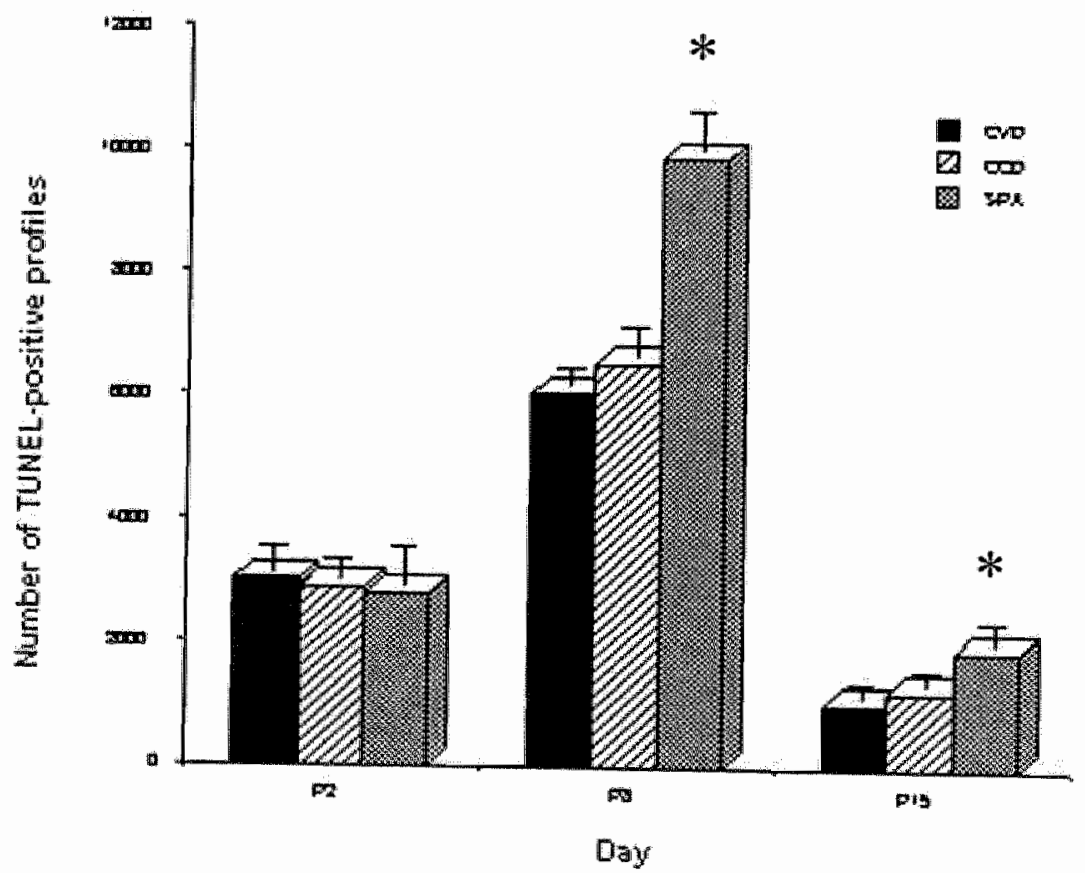

Figure 2. Time course of TUNEL-positive cell profiles during development and after perinatal asphxyia. The total number of apoptotic cells in the striatum was determined by multiplying the sum of TUNEL-stained cell profiles in all sections by the sampling interval (i.e., equal to eight). Data for each rat on each day are expressed as number of dying cell profiles in the left striatum. At this developmental age, there was progressively changing basal level of naturally occurring cell death, peaking at PQ. At P2 following asphyxia, there was no significant increase in levels of cell death in the striatum ( $P>0.05)$. By P8 and P15, a significant increase in the total number of TUNEL-positive cell profiles in the striatum was seen $(P=0.03$ and $P=0.04$, respectively). At all time points, 5 neonates were examined
(total $n=45$ ). 


\section{Stereological analysis}

Table I displays the results of the stereological analysis. The mean total number of neurons in the striatum of control animals, CVD and CCD, was respectively $1,872,394( \pm 183,603 \mathrm{SD})$ and $1,906,495( \pm 162,024 \mathrm{SD})$. By contrast, in the asphyctic animals (SPA) the mean total number of striatal neurons was $1,624,160$ ( $t 152,113 \mathrm{SD}$ ).

The percentage of difference between SPA and CVD or CCD was respectively $15.3 \%(\mathrm{P}=$ $0.03)$ and $17.4 \%(P=0.03)$. The difference between $C V D$ and $C C D$ was only $1.8 \%(P=$ 0.47). Considering Bonferroni correction, the difference between SPA and CVD has to be interpreted as significant but unspecific, and the difference between SPA and CCD as significant and specific. However, this kind of correction is the most conservative one for controlling unacceptable levels of type I error, and the difference between these groups would be considered as specific if the CCD animals would not have been investigated. Therefore is was reasonable to interpret the difference between the groups SPA and CVD also as a significant reduction of the mean total number of striatal cells at $\mathrm{P} 21$.

Table 1. Characteristics of 3 groups of rats (name, number of animals (n), and results found for these animals (i.e., body weight ${ }_{0}$ estimated total number of striatal cells $(\mathrm{m})$, and predicted precision of these estimates).

\begin{tabular}{lrrrr|}
\hline Group & CVD & CCD & SPA \\
Age (days) & & 5 & 6 & 5 \\
Body weight (g) & 21 & 21 & 21 \\
& Mean & 43.1 & 42.3 & 40.8 \\
Est. total number of striatal cells (m) Mean & $1,872,394$ & $1,906,495$ & $1,624,160$ \\
& $\mathrm{SD}$ & 183,603 & 162,024 & 152,113 \\
CEpred.(m) & & & $0.036-0.046$ & \\
& & & & \\
\hline
\end{tabular}

Note. Kruskall-Wallis test: comparison between SPA, CCD, CVD, $P=0.035$. Mann-Whitney$U$ test post-hoc pairwise comparisons between SPA and $C C D, P=0.028$ and between SPA and CVD, $P=0.029$. The total number of neurons in striatum of asphyctic animals is $17.4 \%$ less than in the CCD and $15.3 \%$ less than in the CVD animals.

\section{Discussion}

In this study, we showed that asphyxia leads to cell death in the striatum in the first two weeks of life, with the morphology of apoptosis. Furthermore, the effect was not limited to exaggerated developmental apoptosis, but it reduced the total number of neurons in the rat striatum. In addition, the morphology of the apoptotic cells differed from necrotic pattern observed in other models for perinatal asphyxia $[2,6,28,52]$. The present study emphasizes 
the repeated warning in the literature to use different staining methods for determining cell death or apoptosis in the brain. A combined approach of TUNEL, morphological staining methods and stereology can give clear evidence of the number of dying cells in a defined area. Thus far, there are no studies reporting total numbers of neurons in the rat striatum. In addition, there is no study in the literature analyzing the total number of TUNEL-stained cell profiles during development or following perinatal asphyxia in the rat striatum. To obtain data as valid as possible, all quantification was done applying state-of-the-art methodologies, considering all possible shortcomings, especially with respect to stereology $[20,22,57,58]$. The combined approach using TUNEL and stereology to determine damage due to the immature rat brain enabled us to study the time frame of apoptotic cell death. Since TUNEL can also mean an increased number of DNA-repairing cells, stereological data are necessary to confirm that an increased number of TUNEL-positive cells do really mean, "more cell death" [8].

Apoptosis is an organized, energy-dependent process, characterized by degradation of the cell into membrane-bound fragments. It plays an important role in brain development, as up to $50 \%$ of cells in the developing brain die by apoptosis [49], and there is evidence to suggest that immature cells are more prone to apoptosis than mature cells [4]. The definition and detection of apoptosis is mainly based on two hallmarks of the process: typical morphologic changes $[30,33,38]$ and internucleosomal DNA fragmentation, executed by selectively activated DNAses [16]. The significance of apoptosis has mostly been studied using the TUNEL assay that detects DNA strand breaks in tissue sections and allows quantification of damaged cells by light microscopy [17]. However, common experience has shown that the TUNEL assay cannot be used to distinguish between apoptotic and necrotic cells, since positive cells may contain either laddered or randomly digested DNA and/or active DNA repair systems [8]. The specificity of the results can be substantiated by combining other methods with TUNEL, such as the assessment of the pattern of DNA fragmentation or the evaluation of the morphological features with Hoechst 33324 , HE or confocal microscopy.

Morphologically, apoptosis is characterized by condensation of the nuclear chromatin, the cytoplasm and nucleus, and DNA fragmentation. Necrosis is associated with swelling of the cell, dilation of the endoplasmic reticulum, alteration of the mitochondria and rupture of the plasma membrane $[11,30]$. Furthermore, a third type of morphology has been described in immature rat brain as a consequence of excitotoxicity, designated as vacuolated [51]. These different features of cell death are not exclusive forms of cell death but can coexist in one cell (continuum of different cell types) or brain area [50]. In the present study, an asphyctic period during bith caused degenerative morphology of apoptosis, identical to the apoptosis established during normal brain development. Both condensation and fragmentation were visible in the TUNEL-positive cell profiles following perinatal asphyxia.

The time course of TUNEL staining of striatal neurons showed an increase in apoptosis after asphyxia from $P 2$ to $P 15$. The peak at P8 gives evidence for a delayed form of neuronal death, since in other animal models for perinatal asphyxia and in other regions of the brain, cell death occurs within a few days of the injury $[2,45,63]$. After unilateral hypoxia-ischemia, for example, a peak of induced apoptotic cell death occurs in the substantia nigra 1-2 days after
the injury [48]. It is important to note that the peak in apoptosis is also seen in the injury [48]. It is important to note that the peak in apoptosis is also seen in control animals 
but to a lesser extent; the time of the increase in TUNEL-positive cell profiles therefore seems to be a maturation dependent event [37]. The observed peak in TUNEL-staining at P8 after injury correlates to a reported increase of active caspase-3 levels after injury in P7 pups compared to older pups [25]. Furthermore, it may also be related to the susceptibility of striatal cells to NMDA-mediated toxicity of the immature brain, which peaks around P7 in rats [42]. The NMDA receptor is more active in the immature brain compared to the mature brain and results in excessive calcium influx, activation of second messenger systems, as well as the activation of lipases and caspases $[18,41]$.

The cause of the delayed neuronal death in the striatum after perinatal asphyxia remains unclear. It is hypothesized that asphyxia leads to a sustained alteration of membrane calcium handling, leading to an increase in free cytosolic calcium concentration and gradual calcium overload in the mitochondria $[32,43,61]$. Mitochondrial calcium overload, particularly in association with oxidative stress, can cause delayed cell death. Additional changes in protein synthesis and signaling transduction deprives cells of enzymes or trophic factor essential for survival [60]. Our data show that asphyxia predisposes cells to die especially in the first 8 days after the insult, suggesting that asphyxia triggers a long-lasting reaction which might include mitochondrial damage.

The neuron loss $(-16 \%)$, seen 21 days after perinatal asphyxia, is a direct consequence of cell death induced by the birth asphyxia. Together with the TUNEL data, these data show that cells die as a consequence of perinatal asphyxia and that this process takes place in the first 15 days after birth. Furthermore, it indicates that the cells are lost in an apoptotic manner, since DNA fragmentation is visible in the TUNEL-positive cells (confocal microscopy). Since the difference in TUNEL-positive cells between the groups is much larger that the difference in cell counts using stereology, we hypothesize that not all TUNELpositive cells are lost or that the extensive increase in apoptotic cell death during the first two weeks of life is partly compensated for by neurogenesis.

The difference in total number of neurons in the striatum between asphyctic and control animals was not found by Kohlhauser et al., who studied cell density in the striatum following perinatal asphyxia [31]. This could be a direct consequence of the method applied by Kohlhauser et al., since only one micrograph of each brain region/area of each animal was subjected to the analysis of cell density. Here, more than 600 neurons were counted throughout the brain region per animal, to give an estimate of the total number of neurons in the specific region, i.e. striatum, using state-of-the-art stereology.

Interestingly, the finding of striatal neuron loss here is in line with other reports describing a loss of GABA-ergic and/or cholinergic neurons in the striatum following perinatal asphyxia $[15,31,39]$. Furthermore, a loss of striatal cells could explain the known behavioural changes due to perinatal asphyxia such as hyperactivity, attential deficits and mental retardation and motor disorders. Moreover, it points to potentially important long-term effects of perinatal asphyxia; the involvement of the striatum may help to explain the risk factor for mental disorders, such as dementia and schizophrenia, in humans with a clinical history of perinatal asphyxia $[26,46,64]$. 


\section{Acknowledgements}

We are grateful to Miss Hellen Steinbusch and Dr Hans-Georg Frank for technical assistance. We also thank Dr. Dick Terwel and Prof. Dr. H. Korr for helpful discussions.

\section{References}

[1] Abe, K., M. Aoki, J. Kawagoe, T. Yoshida, A. Hattori, K. Kogure, Y. Itoyama. 1995. Ischemic delayed neuronal death. An mitochondrial hypothesis. Stroke 26: 1478-1489.

[2] Beilharz, E.J., C.E. Williams, M. Dragunow, E.S. Sirimanne, P.D. Gluckman. 1995. Mechanisms of delayed cell death following hypoxic-ischemic injury in the immature rat: evidence for apoptosis during selective neuronal loss. Mol. Brain Res. 29: 1-14.

[3] Berger $R, Y$. Garnier. 1999. Pathophysiology of perinatal brain damage. Brain Res. Rev. 30: 107-34.

[4] Blaschke, A.J., J.A. Weiner, J. Chun. 1998. Programmed cell death is a universal feature of embryonic and postnatal neuroproliferative regions throughout the central nervous system. J. Comp. Neurol. 396: 39-50.

[5] Brown, J.K., F.J. Purvis, J.O. Forfar, F. Cockburn. 1974. Neurological aspects of perinatal asphyxia. Dev. Med. Child Neurol. 16: 567-80.

[6] Burke, R.E., A.L. Karanas. 1990. Quantitative morphological analysis of striatal cholinergic neurons in perinatal asphyxia. Ann. Neurol. 27: 81-8.

[7] Capurso, S.A., M.E. Calhoun, R.R. Sukhow, P.R. Mouton, D.L. Price, V.E. Koliatsos. 1997. Deafferentation causes apoptosis in cortical sensory neurons in the adult rat. J. Neurosci. 17:7372-7384.

[8] Charriaut-Marlangue, C., Y. Ben-Ari. 1995. A cautionary note on the use of the TUNEL stain to determine apoptosis. NeuroReport 7:61-64.

[9] Chen, Y., D. Engidawork, F. Loidl, E. Dell'Anna, M. Goiny, G. Lubec, K. Andersson, M. Herrera-Marschitz. 1997. Short- and long-term effects of perinatal asphyxia on monoamine, amino acid and glycolysis product levels measured in the basal ganglia of the rat. Dev. Brain Res. 104: 19-30.

[10] Chen., Y. S.O. Ogren, B. Bjelke, P. Bolme, P. Eneroth, J. Gross, F. Loidl, M. Herrera-Marschitz, $K$. Andersson. 1995. Nicotine treatments counteracts perinatal asphyxia-induced changes in the mesostriatal/limbic dopamine systems and motor behaviour in the four-week-old male rat. Neuroscience $68: 531-538$.

[11] Clarke, P.G.H. 1990. Developmental cell death: morphological diversities and multiple mechanisms. Anat. Embryol. 181: 195-213.

[12] Colbourne, F., G.R. Sutherland, R.N. Auer. 1999. Electron microscopic evidence against apoptosis as the mechanism of neuronal death in global ischemia. J. Neurosci. 19:4200. 4210.

[13] Delivoria-Papadopoulos, M., Mishra, O.P. 1998. Mechanisms of cerebral injury in perinatal apshyxia and strategies for prevention. J. Pediatr. 132: S30-\$34.

[14] Dell'Anna, E. Y. Chen, E. Engidawork, K. Andersson, G. Lubec, J. Luthman, M. HerreraMarschitz. 1997. Delayed neuronal death following perinatal asphyxia in rat. Exp. Brain Res. 115: 105-115.

[15] Dell'Anna, E., M.C. Geloso, M. Magarelli, M. Molinari. 1996. Development of GABA and calcium binding proteins immunoreactivity in the rat hippocampus following neonatal anoxia. Neurosci. Lett. 211: 93-96. 
[16] Enari, M., H. Sakahira, H. Yokoyama, K. Okawa, A. Iwamatsu, S. Nagata. 1998. A caspaseactivated DNase that degrades DNA during apoptosis, and its inhibitor ICAD. Nature 391 : 396.

[17] Gavrieli, Y., Y. Sherman, S.A. Ben-Sasson. 1992. Identification of programmed cell death in situ via specific labeling of nuclear DNA fragmentation. J. Cell Biol. 119: 493-501.

[18] Ghosh, A. and M.E. Greenberg. 1995. Calcium signaling in neurons: molecular mechansims and cellular consequences. Science 268: 239-247.

[19] Glaser, E.M., Wilson, P.D. 1998. The coefficient of error of optical fractionator population size estimates: a computer simulation comparing three estimators. J. Micros. 192; 163-171.

[20] Guillery, R.W., K. Herrup. 1997. Quantification without pontification: choosing a method for counting objects in sections tissues. J. Comp. Neurol. 386: 2-7.

[21] Gundersen, H.J.G. 1986 Stereology of arbitrary particles. J. Microsc. 143: 3-45.

[22] Hatton, W.J., C.S. von Bartheld. 1999. Analysis of cell death in the trochlear nucleus of the chick embryo: calibration of the optical disector counting method reveals systematic bias. J. Comp. Neurol. 409: 169-186.

[23] Heinsen, H., U. Rub, M. Bauer, G. Ulmar, B. Bethke, M. Schuler, F. Bocker, W. Eisenmenger, M. Gotz, H. Korr, C. Schmitz. 1999. Nerve cell loss in the thalamic mediodorsal nucleus in Huntington's disease. Acta Neuropathol. 97: 613-622.

[24] Herrera-Marschitz, M., C.F. Loidl, Z.B. You, K. Andersson, R. Silviera, W.T. O'Connor, M. Goiny. 1994. Neurocircuitry of the basal ganglia studlied by monitoring neurotransmitter release. Effects of intracerebral and perinatall asphyctic lesions. Mol. Neurobiol. 9: 171-182. Hu, B.R., C.L. Liu, Y. Ouyang, K. Blomgren and B.K. Siesjö. 2000. Involvement of caspase3 in cell death after hypoxia-ischemia declines during brain maturation. J. Cereb Blood Flow Metab. 20: 1294-1300.

[26] Hultman, C.M., P. Sparen, N. Takei, R.M. Murray, S. Cnattingius. 1999. Prenatal and perinatal risk factors for schizophrenia, affective psychosis, and reactice psychosis of early onset: case-control study. BMJ 318: 421-6.

[27] Huppertz, B., H.G. Frank, P. Kaufmann. 1999. The apoptosis cascade-morphological and immunohistochemical methads for its visualization. Anat. Embryol. 200: 1-18.

[28] Johnston, M.V. 1983. Neurotransmitter alterations in a model of perinatal hypoxic-ischemic brain injury. Ann. Neurol. 13: 511-518.

[29] Kerr, J.F.R and B.V. Harmon. 1991. Definition and incidence of apoptosis: An historical perspective. In Apoptosis: The molecular basis of cell death. (L.D. Tomei and F.O. Cope Eds.), pp. 5-29. Cold Spring Harbor Laboratory Press, Cold Spring Harbor, NY.

[30] Kerr, J.F.R., A.H. Wyllie, A.R. Currie. 1972. Apoptosis: a basic biological phenomenon with wide-ranging implications in tissue kinetics. Br. J. Cancer 26: 239-257.

[31] Kohlhauser, C., S. Kaehler, W. Mosgoeller, N. Singewald, D. Kouvelas, H. Prast, H. Hoeger, B. Lubec. 1999. Histological changes and neurotransmitter levels three months following perinatal asphyxia in the rat. Life Sci. 64:2109-2124.

[32] Kristian, T., B.K. Siesjo. 1996. Calcium-related damage in ischemia. Life Sci. 59: $357 \cdot 67$.

[33] Leist, M., Nicotera ${ }_{1}$. 1997. The shape of cell death. Biochem. Bilophys. Res. Commun. 236: $1-9$.

[34] Loidl, C.F., F. Capani, J.J. Lopez-Costa, A. Selvin-Testa, E.M. Lopez, J. Pecci-Saavedra. 1997. Long term changes in NADPH-diaphorase reactivity in striatal and contical neurons following experimental perinatal asphyxia: neuroprotective effects of hypothermia. Int. J. Neurosci. 89: 1-14.

[35] Loidl, C.F., M. Herrera-Marschitz, K. Andersson, Z.B. You, M. Goiny, W.T. O'Connor, R. Silveira, R. Rawal, B. Bjelke, Y. Chen. 1994. Long-term effects of perinatal asphyxia on basal ganglia neurotransmitter systems studied with microdialysis in rat. Neurosci. Lett. $175: 9-12$. 
[36] Loidl, C.F., J. De Vente, M.M. Van Ittersum, E.H.J. Van Dijk, J.S.H. Vhes, H.W.M. Steinbusch, C.E. Blanco. 1998. Hypothermia during or after severe perinatal asphyxia prevents increase in cyclic GMP related nitric oxide levels in the newborn rat striatum. Brian Res. 791: 303307.

[37] Maciejewska, B., M. Lipowska, P. Kowianski, B. Domaradzka-Pytel, J Morys. 1998. Postnatal development of the rat striatum-a study using in situ DNA end labeling technique. Acta Neurobiol. Exp. 58: 23-28.

[38] Maino, G. and 1. Joris. Apoptosis, oncosis, and necrosis. An overview of cell death. Am. J. Pathol. 146: 3-15.

[39] Mallard, E.C. H.J. Waldwogel, C.E. Williams, R.L. Faull, P.D. Gluckman. 1995. Repeated asphyxia causes loss of striatal projection neurons in the fetal sheep brain. Neuroscience 65: 827-836.

[40] Martin, L.J., A. M. Brambrink, A.C. Price, A. Kaiser, D.M. Agnew, R.N. Ichord, R.J. Traystman. 2000. Neuronal death in newborn striatum after hypoxia-ischemia is necrosis and evolves with oxidative stress. Neurobial. Dis. 7: 169-91.

[41] McDonald, J.W. and M.V. Johnston. 1990. Physiological and pathophysiological roles of excitatory amino acids during central nervous system development. Brain Res. Rev. 15: 41-70.

[42] McDonald, J.W. F.S. Silverstein, M.V. Johnston. 1988. Neurotoxicity of N-methyl-D-aspartate is markedly enhanced in developing rat central nervous system. Brain Res. 459: 200-203.

[43] Meyer, F.B. 1989. Calcium, neuronal hyperexcitability and ischemic injury. Brain Res. Rev.. 14:227-243,

[44] Mishra, O.P., M. Delivoria-Papadopoulos. 1999. Cellular mechanisms of hypoxic injury in the developing brain. Brain Res. Bull. 48: 233-238.

[45] Nakajima, W., A. Ishida, M.S. Lange, K.L. Gabrielson, M.A. Wilson, L.J. Martin, M.E. Blue M.V. Johnston. 2000. Apoptosis has a prolonged role in the neurodegeneration after hypoxic ischemia in the newborn rat. $J$. Neurosci. 20: 7994-8004.

[46] Nyakas, C., B. Buwalda, P.G. Luiten. 1996. Hypoxia and brain development. Prog. Neurobiol. 49: 1-51.

[47] Northington, F.J., D.M. Ferriero, D.L. Flock, and L.J. Martin. 2001. Delayed neurodegeneration is neonatal rat thalamus after hypoxia-ischemia is apoptosis. J. Neurosci. 21: 1931-1938.

[48] Oo, T.F., C. Henchcliffe, R.E. Burke. 1995. Apoptosis in substantia nigra following developmental hypoxic-ischemic injury. Neuroscience 69: 893-901.

[49] Oppenheim, R.W. 1991. Cell death during development of the nervous system. Annu. Rev. Neuroscience 14: 453-501.

[50] Portera-Cailliau, C., D.L. Price, L.J. Martin. 1995. Excitotoxic neuronal death in the immature brain is an apoptosis-necrosis morphological continuum. J. Comp. Neuroll. 378: 70-87.

[51] Portera-Cailliau, C., D.L. Price, L.J. Martin. 1997. Non-NMDA and NMDA receptor-mediated excitotoxic neuronal deaths in adult brain are morphologically distinct: further evidence for an apoptosis-necrosis continuum. J. Comp. Neurol. 378: 88-104.

[52] Pulera, M.R. L.M. Adams, H. Liu, D.G. Santos, R.N. Nishimura, F. Yang, G.M. Colle, C.G. Wasterlain. 1998. Apoptosis in a neonatal rat model of cerebral hypoxia-ilschemia. Stroke 29: $2622-2630$

[53] Raff, M.C. 1992. Social controls on cell survival and cell death. Nature 356:397-400.

[54] Raff, M.C, B.A. Barres, J.F. Burne, H.S. Coles, Y. Ishizaki M.D. Jacobson. 1993. Programmed cell death and the control of cell survival: Lessons from the nervous system. Science 262:
$695-700$.

[55] Raichle, M.E. 1993. The pathophysiology of brain ischemia. Ann. Neurol. 13: 2-10.

[56] Schmitz, C. 1998. Variation of fractionator estimates and its prediction. Anat. Embryol. 198: 
[57] Schmitz, C. 2000. Towards the use of state-of-the-art stereology in experimental gerontology. Exp. Gerontol. 35: 429-31.

[58] Schmitz, C., E. Bultmann, M. Gube, H. Korr. 1999. Neuron loss in the mouse hippocampus following prenatal injection of tritiated thymidine or saline. Int. J. Dev. Neurosci. 17: 185-190.

[59] Shaffer, J.P. 1995. Multiple hypothesis testing. Annu. Rev. Psychol. 46: 561-584.

[60] Siesjo, B.K, B. Hu, T. Kristian. 1999. Is the cell death pathway triggered by the mitochondrion or the endoplasmic reticulum? J. Cereb. Blood Flow Metab. 19: 19-26.

[61] Siesjo, B.K., P. Siesjo. 1996. Mechanisms of secondary brain injury. Eur. J. Anaesthesiol. 13: $247-68$.

[62] Taylor, D.L, A.D. Edwards, H. Mehmet. 1999. Oxidative metabolism, apoptosis and perinatal brain injury. Brain Pathol. 9: 93-117.

[63] Towfighi, J., D. Mauger. 1998. Temporal evolution of neuronal changes in cerebral hypoxiaischemia in developing rats: a quantitative light microscopic study. Dev. Brain Res. 109: $169-177$.

[64] Vaillancourt, C. and P. Boksa. 1998. Caesarean section birth with general anesthesia increases dopamine-mediated behaviour in the adult rat. Neuroreport 9: 2953-2959.

[65] Van de Berg, W.D.J., A. Blokland, A.C. Cuello, C. Schmitz, W. Vreuls, H.W.M. Steinbusch, C.E. Blanco. 2000. Perinatal asphyxia results in changes in presynaptic bouton number in striatum and cerebral cortex - a stereological and behavioral analysis. J. Chem. Neuroanatomy 20:71-82.

[66] Vannucci, R.C. 1990. Experimental biology of cerebral hypoxia-ilschemia: relation to perinatal brain damage. Pediatr. Res. 27: 317-326.

[67] Vaux, D.L. 1993. Toward an understanding of the molecular mechanisms of physiological cell death. Proc. Natl. Acad. Sci. USA 90: 786-9.

[68] Wyllie, A.H., J..F Kerr and A.R. Currie. 1980. Cell death: the significance of apoptosis. Int. Rev. Cytol. 68: 251-306. 


\section{Qualitative and quantitative analysis of the ontogeny of apoptotic cell death during postnatal development after birth asphyxia}

W.D.J.Van de Berg, A. Scheepens, H.P.J. Steinbusch, H.W.M. Steinbusch, C.E. Blanco

Experimental Neurology, in press 


\section{Abstract}

Cell death is crucial for normal CNS development and has been shown to occur at high levels during the fetal and early postnatal period. Caspase- 3 has been identified as a key protease in the execution of both neuronal and glial apoptosis. In the present study, we investigated the effect of global asplyyxia at term on the ontogeny of apoptotic cell death during the first 15 days of life. We investigated cell death using the TUNEL assay, immunohistochemistry for Caspase-3, electron microscopy and a functional Caspase-3-like assay to illustrate both qualitatively and quantitatively the progression of developmental cell death during the first 15 days after birth. Birth asphyxia increased the number of TUNEL-positive cells within the striatum and hippocampus, but did not affect the distribution of cell death within these areas. In both control and asphyctic rats, TUNEL and Caspase-3 positive cells were primarily found within the proliferating regions of the striatum, hippocampus and cerebellum. TUNEL-positive cells could be partly identified as astrocytes and oligodendrocytes. Ultrastructural analysis showed primarily the classical features of apoptosis occurring within degenerating cells of asphyctic rats. Birth asphyxia increased Caspase-3-like activity within the hippocampus at P8 and caused a decrease at P11. In contrast, the time course of Caspase-3-like activity within the subventricular zone and cerebellum was unchanged by the birth asphyxia. Neuronal and glial cell death during postnatal development within the hippocampus as a result of birth asphyxia may alter total neuron number and consequently increase the risk for cognitive deficits in later life. 


\section{Introduction}

Human and animal studies have shown that specific cell types within the striatum, hippocampus and cerebellum are particularly vulnerable to a perinatal asphyxial insult. For example, the loss of calbindin-immunoreactivity within cerebellular Purkinje cells was reported in children who suffered from hypoxic-ischemic brain injury as well as in the guinea pig fetus after acute hypoxia [18,21]. Furthermore, oligodendrocyte progenitor cells seem to be particularly vulnerable to hypoxic-ischemic injury in premature infants $[1,28]$. Loss of oligodendrocyte progenitors results in long-lasting disturbance of myelination and an increased risk for periventrular leuckomalacia (PVL) [2]. The vulnerability of the different cell types to perinatal asphyxial insults seems to be partly dependent on the stage of neuronal and glial maturation at the time as well as the type of insult $[38,43]$.

Little is known about the morphological type and time course of neuronal or glial cell death after perinatal asphyxia during the early postnatal period. Dell'Anna and co-workers described a dellayed form of neuronal death with apoptotic features after global asphyxia at term in both the striatum and cerebellum of rat pups [9]. Furthermore, our group reported TUNEL-positive cells within the striatum peaking 8 days after the global asphyctic insult at term [41]. There is however a lack of information on the ultrastructural features of cell death and vulnerability of the different cell types following this model of asphyxia.

The objective of the present study was to characterize, using qualitative methods, which cell type is particularly vulnerable to cell death after global asphyxia at term (P0) and whether these cells exhibited morphological features of apoptosis and/or necrosis using electron microscopy. In addition, we were interested in the time course of caspase-3-like activity after global asphyxia. Previously, it was shown that normal developmental apoptosis reaches its highest levels during the first postnatal week and declines thereafter within the striatum and hippocampus. In contrary, the cerebellum disp $\|$ ays a biphasic peak at P10 and P2 1 [45]. We hypothesize that caspase-3-like activity in affected areas is increased after global asphyxia, in particularly within the first week after injury (birth).

\section{Materials and methods}

\section{Induction of global asphyxia at term}

Global asphyxia was induced in rat pups at term as described previously in detail by Loidl et al. [22]. Briefly, time-pregnant Wistar dams were purchased from Charles River-Broekmans (Someren, The Netherlands). They were housed in the Animal Care Department of the University Maastricht under standard conditions $\left(12: 12 \mathrm{~h}\right.$ light:dark cycles, $20^{\circ} \mathrm{C}$, with free access to standard laboratory chow and water). Dams were euthanased by decapitation immediately after delivery of one or two pups (control pups). Afterwards, dams were rapidly hysterectomized and the uterus horns containing the remaining pups were detached and placed in a water bath at $37^{\circ} \mathrm{C}$ for $20 \mathrm{~min}$ (time of perinatal asphyxia). Subsequently, pups 
were removed from the uterus horns and stimulated to breath by cleaning and by gently padding them on the chest in a pediatric incubator $\left(37^{\circ} \mathrm{C}\right.$ and $90 \%$ humidity). The pups were left to recover for $60 \mathrm{~min}$ in the incubator, marked and then placed with a surrogate dam. The percentage of mortality in the SPA group was $\pm 40-60 \%$. All pups belonging to the control groups were gasping immediately after birth and all survived. Only male pups were used for this study since previous studies showed behavioural deficits related to the asphyctic insult occurred primarily in male pups [22]. Animal care and all experimental procedures were approved by the ethical board of Maastricht University.

\section{Tissue preparation}

Control and asphyctic rat pups were randomly assigned to different time points, i.e. P2, P5 or P8 and used for either light microscopic $(n=5$ per group at all time points) or electron microscopic analysis ( $\mathrm{n}=4$ for each group at all time points). For terminal deoxynulceotidyl transferase (TdT)-mediated dUTP nick-end labeling (TUNEL) assay and immunohistochemical analysis, pups were anaesthetized with sodium pentobarbital ( 60 $\mathrm{mg} / \mathrm{kg}$; i.p.; Nembutal) and perfused transcardially $(50 \mathrm{mmHg})$, first with tyrode, followed by the fixative containing $4 \%$ paraformaldehyde and $2 \%$ picric acid in $0.1 \mathrm{M}$ phosphate buffer ( $\mathrm{pH} 7.6)$ at room temperature. The brains were removed from the skull and post fixed for $24 \mathrm{~h}$ in $4 \%$ paraformaldehyde in $0.1 \mathrm{M} \mathrm{PB}$ at $4^{\circ} \mathrm{C}$. Brain tissue was cryoprotected by immersion in $15 \%$ sucrose $/ 0.1 \mathrm{M}$ Tris buffered saline (TBS) for $48 \mathrm{~h}$ at $4^{\circ} \mathrm{C}$. Afterwards, the brains were quickly frozen by placement in isopentane for $25 \mathrm{sec}$, cooled with liquid nitrogen. The brains were entirely cut to serial, $16 \mu \mathrm{m}$ thick, coronal sections on a cryostat (Leica $\mathrm{CM} 3050$ ) and these were stored at $-80^{\circ} \mathrm{C}$ until further processing.

For electron microscopy, pups were perfused through the heart using $4 \%$ paraformaldehyde and $2 \%$ glutaraldeyde in $0.1 \mathrm{M} \mathrm{PB}$ and tissue was post fixed in the same fixative for 3 days. The brains were removed from the skull after post fixation. Samples of the striatum and cerebellum from both hemispheres were microdissected and processed for electron microscopy.

\section{TUNEL labeling}

Every eighth section was used for TUNEL staining using the method developed by Gavrieli et al. (1992), slightly modified to accommodate frozen sections of pup tissue as described previously [41]. Briefly, sections were permeabilized for $10 \mathrm{~min}$ using methacarn (methanol: chloroform: acetic acid $=66: 33: 1 ; \mathrm{v} / \mathrm{v} / \mathrm{v})$, rinsed in TBS $(2 \times 10 \mathrm{~min})$ and incubated in terminal transferase (TdT) buffer (1 M potassium cacodylate, $125 \mathrm{mM}$ Tris, Bovine serum Albumin (BSA) $1.25 \mathrm{mg} / \mathrm{ml}$ and $25 \mathrm{mM}$ cobalt chloride $\left(\mathrm{pH} 6.6,25^{\circ} \mathrm{C}\right), \mathrm{TdT}(0.3 \mathrm{e} . u . / \mu \mathrm{l})$ and biotinylated dUTP $(0.04 \mathrm{mM})$ (Roche, Germany) in a humid chamber at $37^{\circ} \mathrm{C}$ for $90 \mathrm{~min}$. Sections were covered with $20 \mu \mathrm{l}$ of the incubation solution and coverslipped with parafilm. The reaction was stopped by placing the slides in 2 XSC buffer $(300 \mathrm{mM}$ sodium chloride, $30 \mathrm{mM}$ sodium nitrate; $\mathrm{pH} 7.6$ ) followed by TBS for $2 \times 10 \mathrm{~min}$. Subsequently, streptavidin-Cy3 (1:2000 
dilution; Jackson ImmunoResearch Laboratories, USA) was used to visualize nucllei labeled with biotinylated dUTP. After washing in TBS, sections were counterstained with Hoechst 33324 (1:500 dillution; Sigma, USA), rinsed again in TBS and mounted with TBS:glycerol $(1: 3)$

\section{Double labeling}

IUNEL-stained sections were processed for immunohistochemistry to study the colocalization of TUNEL with cell-specific markers. TBS $(0.01 \mathrm{M})$ with $0.2 \%$ Triton X-100 (TBS-T) was used for washing and diluting antibodies. An antibody against glial fibrillary acidic protein (anti-GFAP) (1:1600; Sigma) and vimentin $(1: 1 ;$ Zymed, USA) was used to identify astrocytes. Oligodendrocytes were detected with a monoclonal antiserum against O4 (1:50; MAB345, Chemicon International, USA). Negative controls were performed by omitting the primary antibody. After TUNEL-staining, sections were incubated with primary antibodies overnight at $4^{\circ} \mathrm{C}$. Subsequently, sections were incubated with the secondary antibody (goat anti-mouse Alexa Fluor@ 488 (1:100; Mol. Probes, USA) and coverslipped with TBS:glycerol (1:3) after washing.

Sections were examined at a magnification of $x 400$ and $x 1000$ with an Olympus $\mathrm{AX}-70$ microscope. Images of the sections were captured using a cooled $C \mathrm{CD}$ video camera attached to the microscope which was equipped with a narrowband MNIBA-type FITC filter, or MNG filter for CY fluorescence (Chroma Technology, The Netherlands). Using the Image Analysis System (SIS), grey scaled images were directly converted into artificial colors. All sections were stained simaltaneously and recorded on the same day to minimize experimental variation.

\section{Immunohistochemistry for caspase-3}

Sections adjacent to the TUNEL-stained sections were processed for caspase 3 immunohistochemistry. Prior to incubation with the primary antibody, sections were immersed in $70 \%$ methanol for $10 \mathrm{~min}$ and washed with TBS. Sections were incubated with a rabbit polyclonal antibody directed against caspase-3 (67341 A; Phamingen, Europe) diluted 1:500 in TBS-T. The anti-caspase-3 antibody recognizes both $32 \mathrm{kDa}$ proenzyme and the 12,17 and $20 \mathrm{kDa}$ active fragments (Pharmingen). The caspase-3 antibody was visualized using a biotinylated goat anti-rabbit antibody (1:400; Jackson Immunoresearch Laboratories) and streptavidine Cy-3. Sections were coverslipped using TBS:glycerol (1:3).

\section{Quantification of TUNEL and caspase-3 positive cells}

TUNEL and caspase-3 positive cells were counted in tissue of six rats of each group (control and asphyctic) at $\mathrm{P} 8$ as described previously in Van de Berg et al. (see chapter 2). All TUNELpositive cells were counted regardless of morphology in the striatum, pyramidall cell layer of the hippocampus and granule cell layer of the cerebellum. The total number of TUNEL-positive 
cells was estimated by multiplying the number of counted cells in all sections by the sampling interval (i.e., equal to eight).

\section{Ultrastructural analysis of dying cells within striatum, hippocampus and cerebellum} After fixation the samples were processed for transmission electron microscopy using established techniques. The samples were washed twice with $0.1 \mathrm{M}$ phosphate buffer, postfixed for one hour with $1 \%$ osmiumtetroxide in $0.1 \mathrm{M}$ phosphate buffer, dehydrated in a graded series of ethanol and embedded in epon 812 . Semithin sections of 1 im were cut on a ReichertJung ultracut, stained with $1 \%$ toluidin blue dissolved in $1 \%$ borax, and screened. An area of interest was selected, ultrathin sections (approx.70 nm) were cut and collected on 75 mesh formvar coated copper grids, subsequently the grids were contrasted with uranylacetate in $50 \%$ ethanol for 10 minutes followed by a 5 minute lead citrate staining. The contrasted sections were examined using a Philips CM 10 microscope at $80 \mathrm{KeV}$.

\section{Fluorometric assay of Caspase-3-like activity}

Another set of control and asphyctic rat pups was used for analyses of Caspase-3-like activity at P2, P5 or P8, P1 1 and P15. The pups were euthanased by decapitation at the appropriate time points. The brains of the pups were carefully removed and microdissected using the method of Wagner et al. [44]. The sub ventricular zone (SVZ) within the rostral forebrain, the hippocampus and the cerebellum were collected, weighed and snap frozen in liquid nitrogen. Tissue samples were stored at $-80^{\circ} \mathrm{C}$ until analysis. All dissection was done by the same experimenter to prevent inconsistency.

The tissue samples were homogenized in a lysis buffer containing $137 \mathrm{mM} \mathrm{NaCl}, 20 \mathrm{mM}$ Tris$\mathrm{HCl}(\mathrm{pH} 8.0), 1 \% \mathrm{NP}-40,10 \%$ glycerol and a complete protease inhibitor tablet (Roche, NL). The tissue samples of SVZ, hippocampus or cerebellum were homogenized in $1 \mathrm{ml}$ or $250 \mathrm{ml}$ of homogenization buffer using a Mini-Bead Beater (Biospec products, USA) thrice for 30 seconds with cooling on ice between runs. The tissue samples were briefly centrifuged and an aliquot of the supernatant $(30$ or $50 \mathrm{ml})$ were taken to determine the Caspase-3-like activity.

We used a fluorometric assay to measure Caspase-3-like activity within the homogenates as described previously by Puka-Sundvall and co-workers $[32,33]$. Briefly, samples of homogenates were mixed with extraction buffer 1 ( 70 or $50 \mathrm{ml}$ ) contaning $50 \mathrm{mM}$ Tris-HCL (pH 7.4), $100 \mathrm{mM} \mathrm{NaCl}, 5 \mathrm{mM}$ EDTA, $1 \mathrm{mM}$ EGTA, $3 \mathrm{mM} \mathrm{NaN3}, 1 \mathrm{mM}$ PMSF, $1 \mathrm{mg} / \mathrm{ml}$ pepstatin, $2.5 \mathrm{mg} / \mathrm{mll}$ leupeptin, $10 \mathrm{mg} / \mathrm{ml}$ aprotinun and $0.2 \% \mathrm{CHAPS}$, on a 96 wells microtiter plate (Microflwor; Dynatech, VA). After a 15 min incubation period at room temperature, 100 ml of peptide substrate, $50 \mathrm{mM}$ Ac-Asp-Glu-Val-Asp-AMC (Ac-DEVD-AMC; Biomol, Gemany) in extraction buffer 2 (extraction 1 without inhibitors and CHAPS, but with $4 \mathrm{mM}$ DTT) was added. Cleavage of the Ac-DEVD-AMC was measured at $37^{\circ} \mathrm{C}$ using a Spectramax Gemini microplate fluorometer (Mol. Devices) with an excitation wavelength of $380 \mathrm{~nm}$ and an emission wavelength of $440 \mathrm{~nm}$. The cleavage was followed at $2-\mathrm{min}$ intervals for $3 \mathrm{~h}_{*}$ and 
Vmax was calculated from entire linear part of the curve (as described by supplier). DEVD cleavage was expressed as AMC fluorescence (UFA) per mg of wet weight and minute. Measurement of protein concentration, as described by Whitaker and Granum [46], was not possible due to the small amounts of tissue available at P2, P5 and P8.

\section{Statistics}

Data regarding the number of TUNEL and caspase-3 positive cells were analyzed using Student's t-test. The differences in Ac-DEVD-AMC cleavage between control and asphyctic rats were evaluated using a pairwise two-way ANOVA analysis and post-hoc tests using Bonferroni. correction for repeated measures. Statistics were carried out using SigmaStat ${ }^{\mathrm{TW}}$ software version 2.03. Differences were considered significant if $\mathrm{P}=0.05$. All data are presented as mean $t$ standard error of the mean (SEM).

\section{RESULTS}

\section{Qualitative analysis of TUNEL-positive cells}

Light microscopy analysis of brains from asphyctic rat pups did not reveal any infarcted areas within the striatum, hippocampus of cerebellum at any timepoint. TUNEL-and caspase-3 positive cell profiles were visible in both the grey and white matter of striatum and cerebellum of control and asphyctic pups during the first week of postnatal life. The distribution of TUNELpositive cells within the asphyctic rats was similar to the control rats within the examined regions. Figure 1 illustrates the distribution of TUNEL-positive cells with the striatum, hippocampus and cerebellum of asphyctic rats at P8.

At P2 and P5, TUNEL-positive cells were found mainly within the subventricular zone and the dorsolateral part of the caudate putamen (CPu), whereas at P8 TUNEL-positive cells were more diffusely distributed throughout the entire striatum. TUNEL and caspase-3 positive cell profiles were visible in the corpus callosum at all ages (especially at P2). These cells exhibited different morphological features, e.g.elaborate cell-processes, compared to the TUNEL positive cells within the grey matter. In the hippocampus, TUNEL and caspase-3 positive profiles were present in the pyramidal cell layer (CA1-CA3), as well as in the surrounding areas of the pyramidal cell layer at all time points. Furthermore, TUNEL and caspase-3 positive cell profiles were observed within the fimbria of the hippocampus and in the dentate gyrus. In the cerebellum, TUNEL and caspase 3-positive cells were found within the white matter and in the granular cell layer at P2 and P5. At P8, TUNEL- and caspase 3 positive cells were mainly observed within the granular cell layer. At all time points, TUNEL and caspase-3 positive cell profiles were also observed with in the cingulate cortex.

Previously, we have shown that global asphyxia at term induces an increase in TUNEL-postive cells within the striatum at P8 and P15 [41]. Although, we counted approximately $980 \pm 78$ 
TUNEL-positive cells within the striatum of asphyctic rats at P8, we only counted $712 \pm 59$ ($27 \% ; \mathrm{P}<0.05$ ) caspase- 3 positive cells within adjacent sections of the striatum of these rats. This difference between TUNEL and caspase- 3 positive cells was also observed within the other examined areas (data not shown).
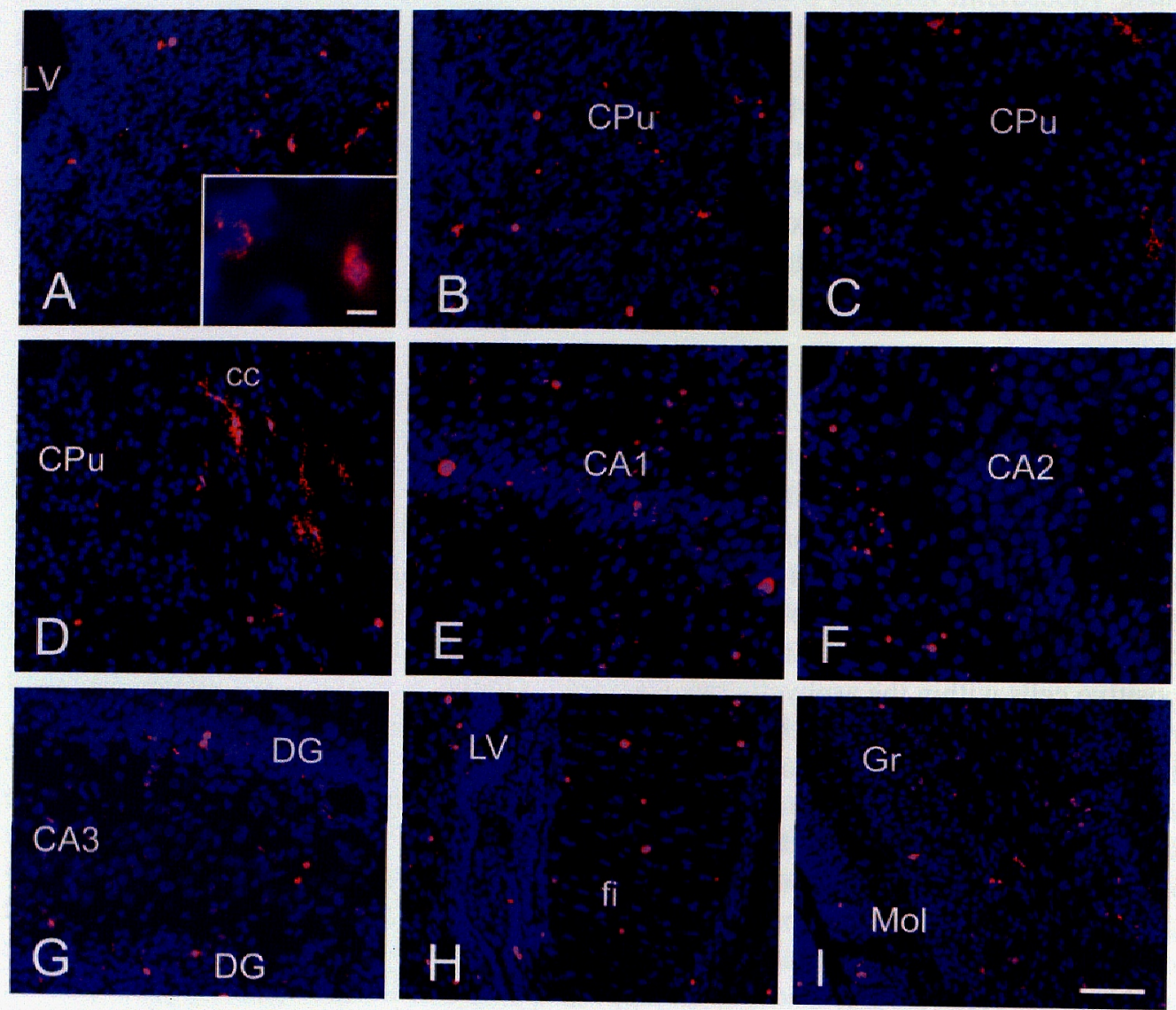

Figure 1. Photomicrographs of TUNEL-stained sections of the SVZ (A,B and $H)$, caudate putamen (A, B, C and D), hippocampus (E,F and $G$ ) and cerebellum (I) of asphyctic rats at postnatal age 8. All sections were counterstained with Hoechst 33324 (blue) to reveal cytoarchitecture. A) area next to the lateral ventricle (LV) showing TUNEL-positive cell profiles (red). B) medial-ventricular part of caudate putamen (Cpu). C) dorso-lateral part of the CPu. D) TUNEL-positive cell profiles within the corpus callosum (cc) exhibiting gliallike morphology. E) TUNEL-positive cell profiles within the CA1 regions of the hippocampus and outer regions of the pyramidal cell layer. F) TUNEL-positive cell profiles in the border zone of the CA2. G) TUNEL-positive cell profiles within the dentate gyrus (DG) and CA3 region of the hippocampus. H) TUNEL-positive cell profiles within the fimbria (fi) of the hippocampus next to the lateral ventricle (LV). I) TUNEL-positive cell profiles within the white matter and granule cell layer of the cerebellum. Scale bars: A-I, $40 \mathrm{~mm} ; \mathrm{J}, 5 \mathrm{~mm}$. 

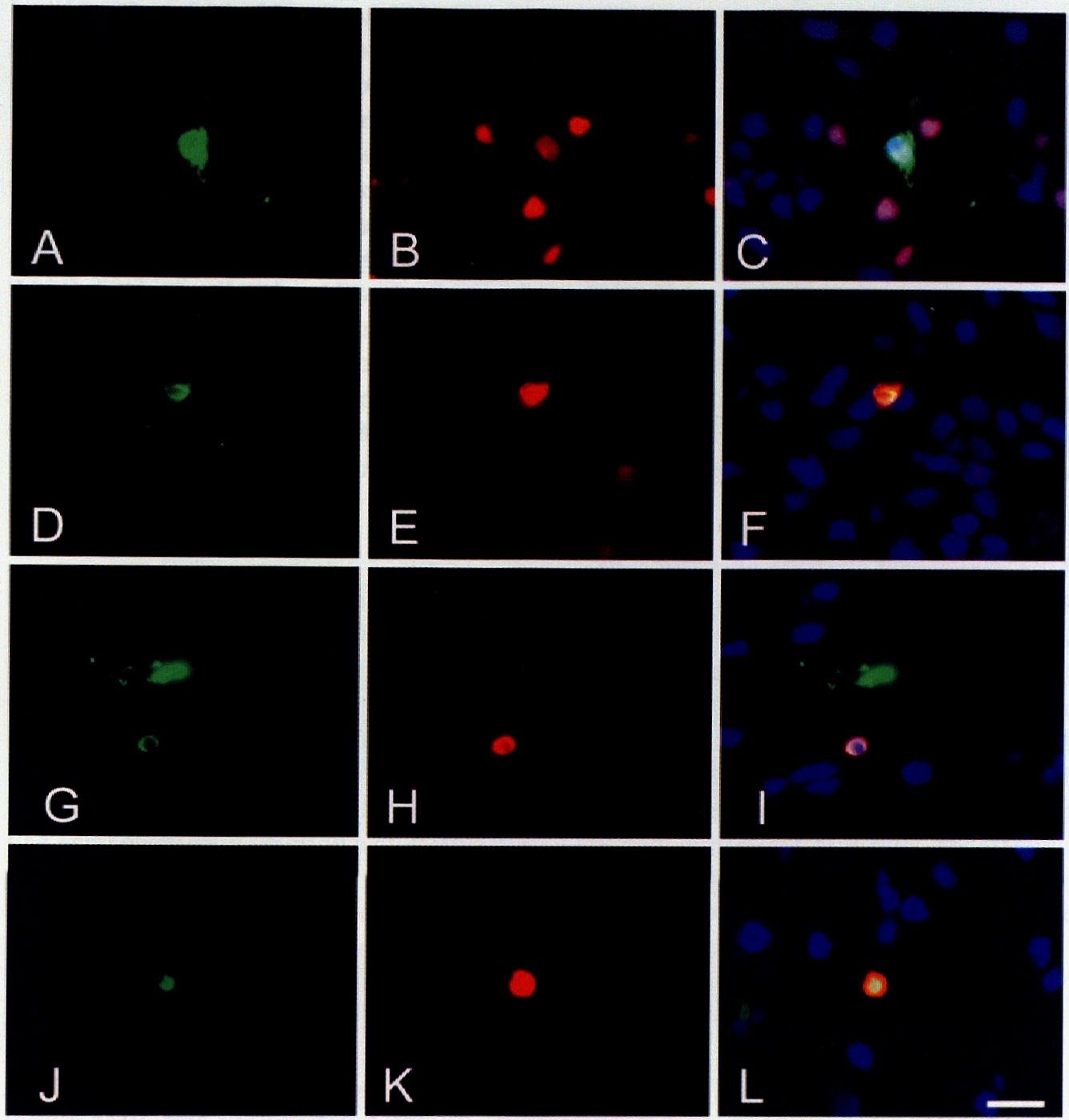

Figure 2. Photomicrographs illustrating co-localization between TUNEL and cell-specific markers, GFAP (astrocytes) or 04 (oligodendrocytes). A and D) GFAP immunoreactivity within the white matter of respectively the cerebellum and the corpus callosum. $\mathrm{G}$ and $\mathrm{J}$ ) O4 immunoreactivity within respectively the cerebellum and the corpus callosum. $\mathrm{C}$ and $\mathrm{F}$ ) co-localization between GFAP and TUNEL, indicative of a dying mature astrocyte. I) no co-localization was observed within the cerebellum between $\mathrm{O} 4$ and TUNEL. J) coIocalization between $\mathrm{O} 4$ and TUNEL within the corpus callosum, indicative of a dying immature oligodendrocyte. Scale bar: $10 \mathrm{~mm}$. 


\section{Co-localization between TUNEL and cell-specific markers}

Double labelling with cell-specific markers revealed TUNEL-staining to co-localize with astrocytes (GFAP-inmunoreactive cells) and immature astrocytes (vimentin-immunoreactive cells) within the white matter of the corpus callossum and cerebellum and within the grey matter of all areas examined in control and asphyctic rats. The 04 antibody labeled both the plasma membrane and cytoplasm of oligodendrocytes. Photomicrographs illustrating the colocalization between TUNEL and cell-specific markers are shown in figure 2 .

Co-localization with TUNEL and 04 was visible within the corpus callossum and the white matter of the cerebellum. Only a wery small percentage (approximately $5 \%$ ) of the TUNELpositive cells, however, could be identified as being either astrocytes or oligodendrocytes in control rats. In asphyctic rats, more TUNEL-positive cells showed co-localization with GFAP (approximately $10-15 \%$ ) or 04 (approximately 10-20\%) than in control rats. Moreover, TUNEL-positive cells within the white matter of asphyctic rats showed a primarily glial-like morphology, i.e. a cell body with many processes. TUNEL-positive neuron-like cells were observed within the pyramidal cell layer of the hippocampus and granule cell layer of the dentate gyrus and cerebellum (see Figure 1 E and II).

\section{Ultrastructural analysis of dying cells}

Ultrastructural analysis of dying cells within the areas examined (striatum, hippocampus and cerebellum) revealed the classic features of apoptosis as described previously by others $[20,31]$ in both neurons and glial cells of control rats. Both condensed and/or fragmented nuclei were observed in all three areas (Figure 3). Organelle and plasma integrity were preserved at early stages of apoptosis in control rats (see Figure 4A). Dying cells within the striatum, hippocampus and cerebellum of asphyctic rats also exhibited the classical features of apoptosis (Figure 3). Furthermore, most neurons and glial cells displayed a normal ultrastructural appearance. However, some mitochondria within cells with a normal appearance (size and structure) were extremely dilated and exhibited fewer cristae. This type of swollen mitochondria was only found within the hippocampus of asphyctic rats at P5 or P8 (Figure 4C). In addition, the endoplasmic reticulum exhibited a swollen appearance in some cells within the hippocampus at P5 and P8 and striatum at P5 (Figure 4D). The plasma membrame integrity and organelles of cerebellar cells seemed to be less affected.

\section{Quantitative analyses of caspase-3-like activity during development and after global
asphyxia}

To assess the time course of caspase-3-like activity during normal development and after global asphyxia, we measured DEVD-cleavage in homogenates of the subventricular zone, hippocampus and cerebellum of control and asplyctic rats during the first 15 days of postnatal life. The results of DEVD-cleavage activity are shown in Figure 5.

DEVD cleavage activity was detectable at all time points in both groups and decreased after PS in the SVZ and hippocampus. Constitutive DEVD cleavage activity in the SVZ peaked at 
$\mathrm{P} 5$, and showed a strong reduction by $\mathrm{P} 15$ (P2 vs. P15 -63\%; P<0.001). Constitutive DEVD cleavage activity in the hippocampus decreased gradually over time and was reduced to $56 \%$ by $\mathrm{P} 15$ ( $\mathrm{P} 2$ vs. P15 $-54 \% ; \mathrm{P}<0.001$ ). The pattern of DEVD cleavage in the cerebellum was different compared to the other regions in that it was more stable during the first week of life, dropped at P1 1 (P8 vs. P1 $1 ; \mathrm{P}<0.01$ ) and than increased again at P15.

The time course of DEVD cleavage activity was not different between control and asphyctic group in the SVZ and cerebellum, in contrary to the time course of DEVD cleavage activity within the hippocampus, which was altered by global asphyxia. DEVD cleavage activity within the hippocampus remained at high levels until P8 in the asphyctic group (group $x$ time; $\mathrm{P}=0.01$ ). Furthermore, global asphyxia caused an increase in DEVD cleavage activity within the hippocampus at $\mathrm{P} 8(\mathbb{P}=0.02)$, and a decrease at $\mathrm{P} 11(\mathrm{P}=0.02)$. At $\mathrm{P} 2$, a small decrease in DEVD cleavage after global asphyxia was observed, this was however not significant $(P=0.06)$. Figure 6 shows a comparison of DEVD cleavage activity between control and asphyctic groups for the SVZ, hippocampus and cerebellum. This figure clearly illustrates that global asphyxia did not affect DEVD-cleavage activity during the first days after injury, but instead caused delayed changes in DEVD-cleavage activity during the second week of postnatal life.
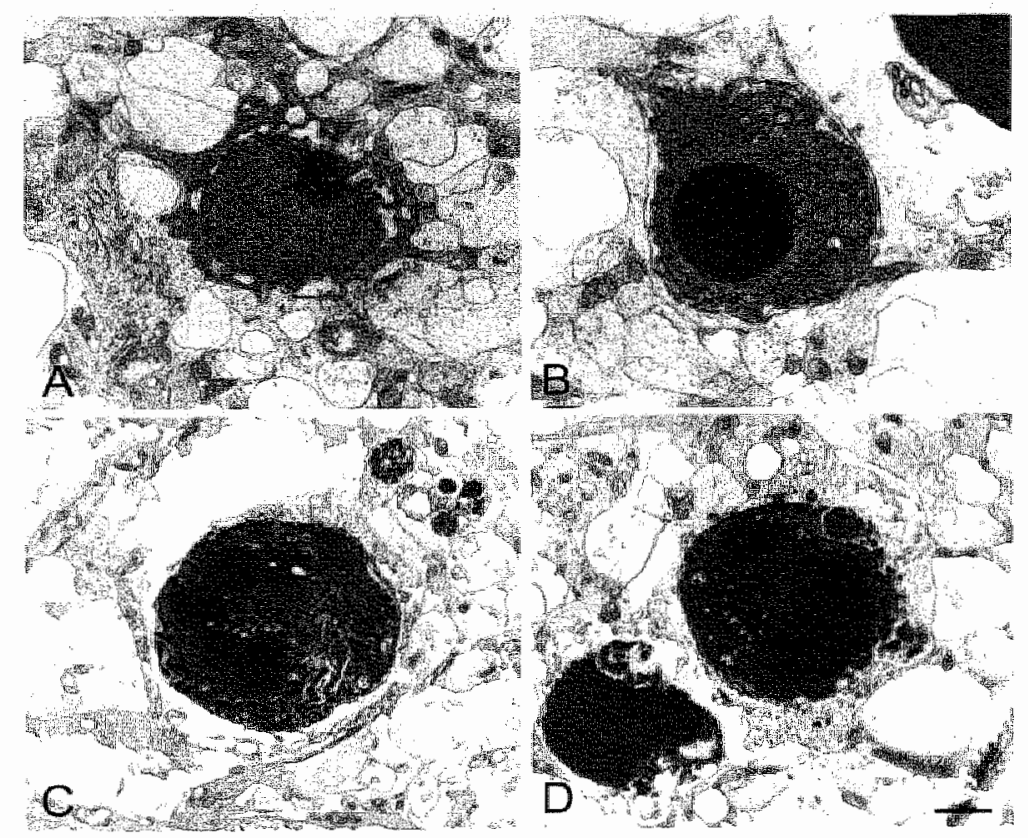

Figure 3. Examples of electron micrographs illustrating morphological features of dying cells within asphyctic rats. Cells exhibited classical features of apoptosis. A and B) these photomicrographs illustrate shrinkage of nucleus and chromatin condensation. $C$ and $D$ ) nuclear condensation (C) and formation of apoptotic bodies (D). Scale bars: A,D $3.8 \mathrm{~mm}$; $\mathrm{B}, 2.2 \mathrm{~mm} ; \mathrm{C}, 0.7 \mathrm{~mm}$. 


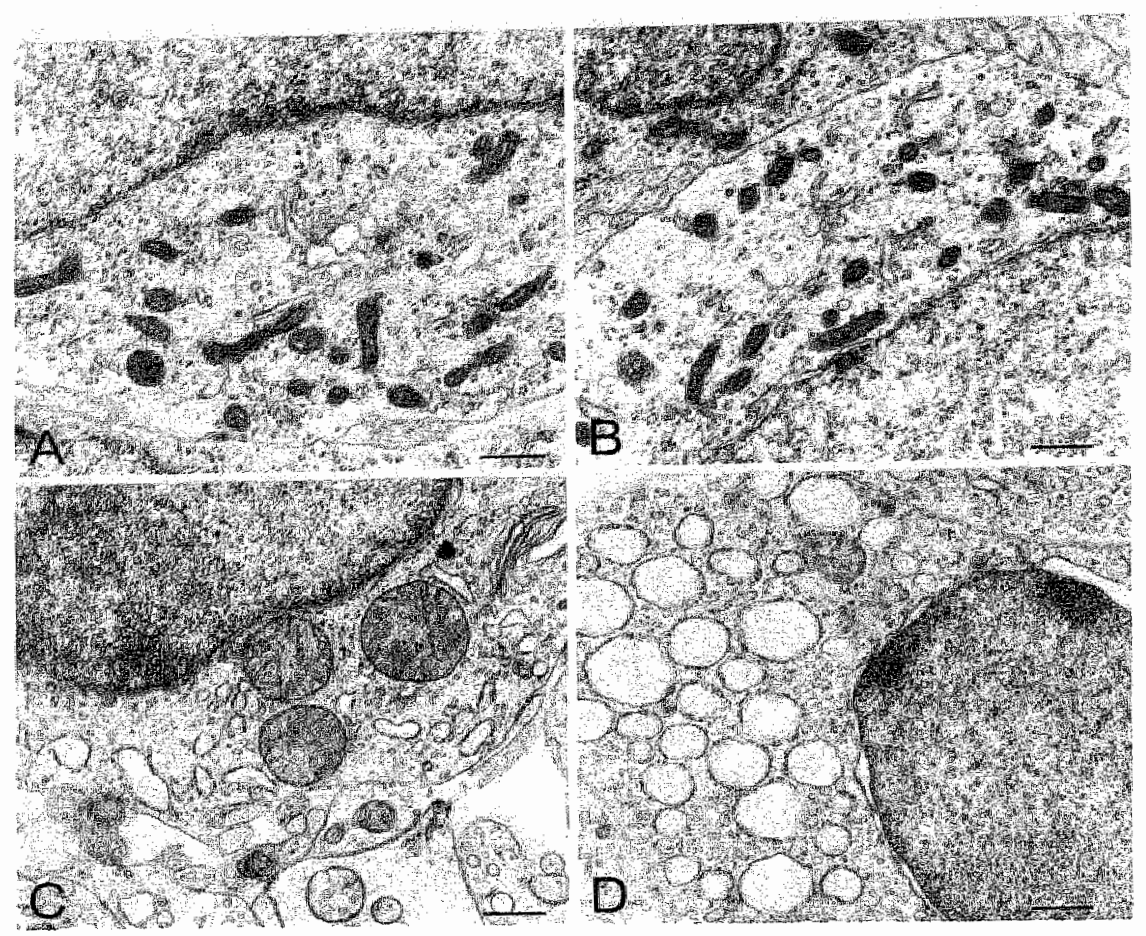

Figure 4. Photomicrographs of the striatum of a control rats (A) and striatum and hippocampus of asphyctic rat $(B, C, D)$ at $P 8 . A, B)$ Mitochondria and endoplasmic reticuli within the striatum of control (A) and asphyctic (B) rats with a normal appearance. C) Swollen mitochondria within hippocampal neuron-like cells with loss of cristae. D) Swollen endoplasmatic reticuli within hippocampus of asphyctic rats at P8. Scale bars: A, B 0.3 $\mathrm{mm} ; \mathrm{C}, \mathrm{D} 2.2 \mathrm{~mm}$.

\section{Discussion}

In the present study, we showed that both neurons and glial cells in specific areas of the SVZ, hippocampus and cerebel tum exhibit apoptotic features during the 15 days of postnatal life in both nomal and asphyctic rats. Global asphyxia at term did not affect the distribution of TUNEL and Caspase-3 positive cells but significantly increased the number of TUNEL. positive cells within the striatun and hippocampus, but not within the cerebellum. Furthermore, our results demonstrate that Caspase-3-like activity, which is associated with apoptotic cell death, is high during the first week of postnal development and declines afterwards in the SVZ and hippocampus in both control and asphyctic rats. The Caspase-3-like activity in the SVZ presumably corresponds with the appearance of apoptotic neurons and glial cells (TUNEL and Caspase 3-positive cells) within the ventricular zone and dorsolateral part of the striatum, 
corpus callossum and sparse neurons within the cortex. Whereas Caspase-3-like activity within the hippocampus likely corresponds to glial cell death within the regions surrounding the pyramidal cell layer, granule cells in dentate gyrus and cells in the subventricular zone. The pattern of Caspase-3-like activity in the cerebellum is unique in that it declines after the first week of postnatal life, but increases again afterwards (P15).

Global asphyxia at term increased Caspase-3-like activity within the hippocampus at P8, which may reflect an increase in neuronal death, as TUNEL-positive cells were observed within the pyramidal cell layer (primarily in CA1 -CA2) only after asphyxia. The decrease in Caspase-3like activity at PI 1 within the hippocampus after global asphyxia, however, might indicate a spatial shift in the pattern of Caspase-3-like activity during normal development within the hippocampus rather than an increase in total number of dying cells.

\section{Distribution of apoptotic cell in the CNS after global asphyxia}

Apoptotic cell death is crucial for the normal development of the CNS and occurs in all brain areas during fetal and postnatal life [4,24]. During the fetal period and early postnatal life, widespread apoptosis has been shown mainly in proliferative regions of the CNS [5]. These regions include the sub ventricular zone (SVZ), the subgranular layer of the dentate gyrus and the granular cell layer of the cerebellum [46]. Afterwards, apoptosis occurs primarily in postmitotic cells throughout adulthood. The results from our study clearly illustrate the large magnitude of cell death within proliferative regions of the CNS during early postnatal development within control and asphyctic rats. These data support previous studies showing a large amount of postnatal neurogenesis and postnatal cell death within the SVZ, hippocampus and cerebellum $[3,45,46]$.

In addition, we showed that selected neurons, astrocytes and oligodendrocytes exhibit apoptotic features during postnatal development. Recent reports provided evidence for an active regulatory role of mature astrocytes in neurogenesis and synapse formation during development [39]. In addition, oligodendrocytes not only posses myelinating properties, but provide trophic support and are able to effect neuronal development [16]. Cell death of astrocytes and oligodendrocytes during postnatal development may therefore play an important role in determining the total number of neurons in later life. Global asphyxia at term increased the number of TUNEL-positive astrocytes and oligodendrocytes within the corpus callosum and cerebellum. Therefore, global asphyxia may not only alter total number of neurons directlly by inducing death, but also by inducing glial loss and thereby causing loss of support by astrocytes and oligodendrocytes.

\section{The ontogeny of apoptotic cell death after global asphyxia at term}

The ontogeny of apoptosis during early postnatal development has primarily been studied by counting pyknotic cells (cresyl violet or silver impregnation method) or TUNEL-positive cells within the brain $[5,13,23]$. Further, the pattern of physiological cell death has been studied using an ELISA for oligonucleosomal DNA fragments [45] and a quantitative immunoblotting 


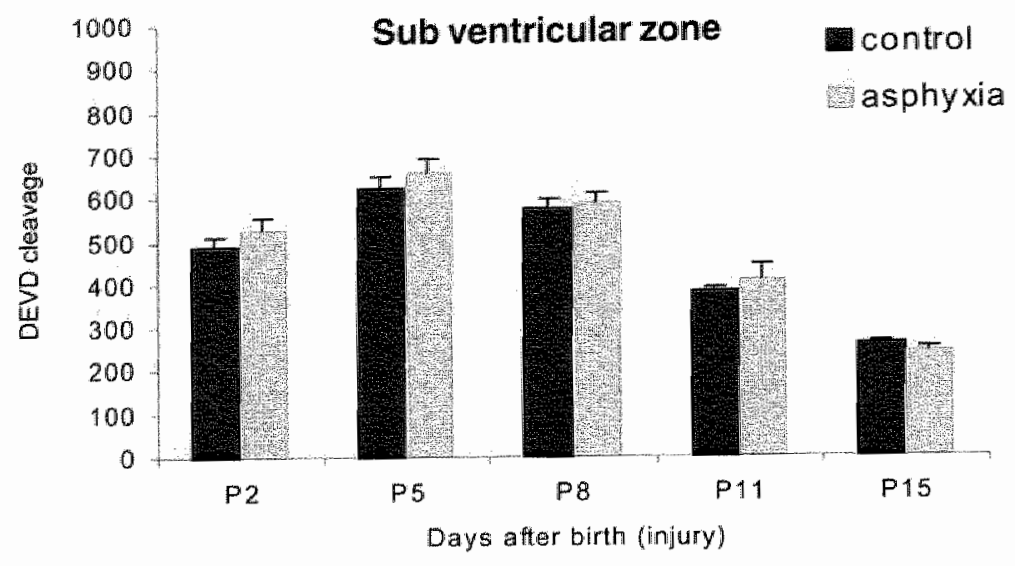

Hippocampus
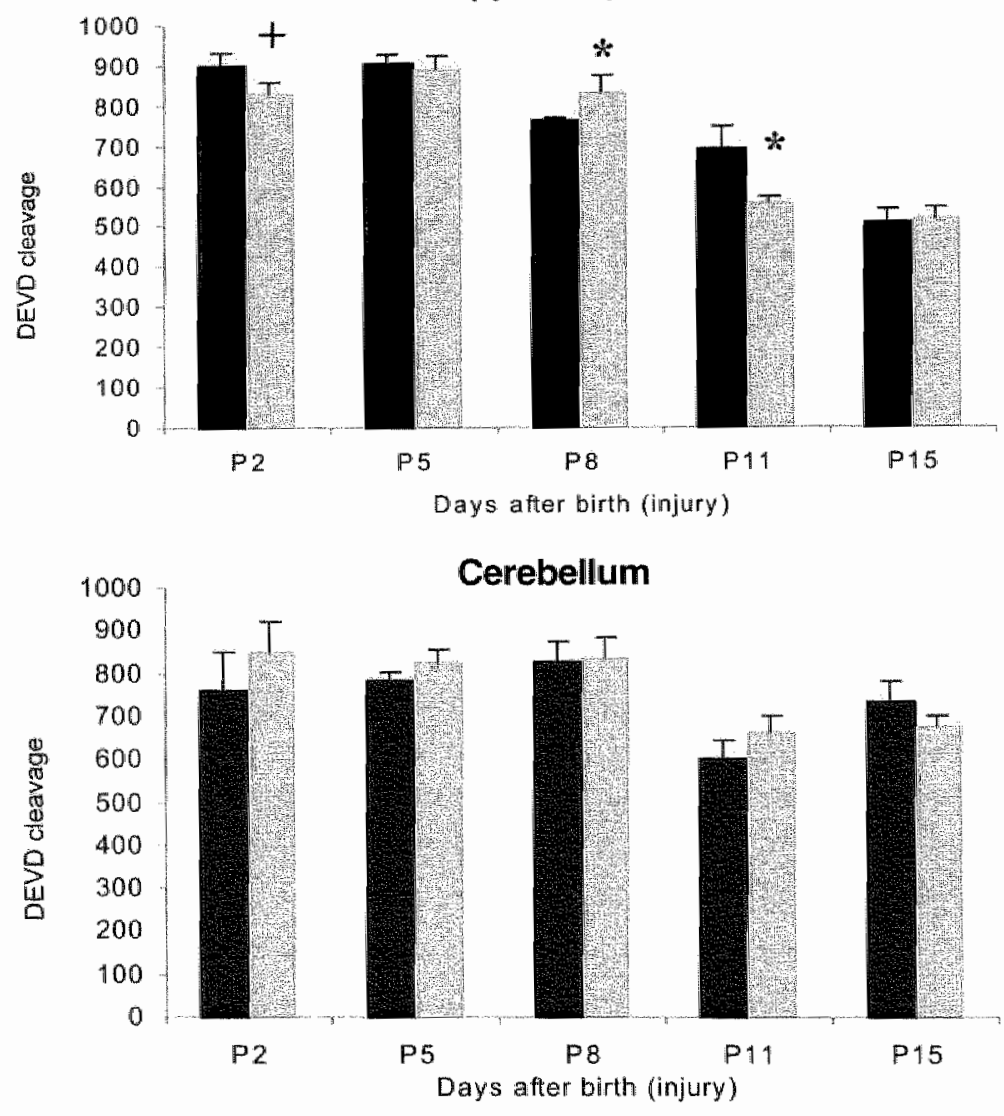

Figure 5. Caspase-3-like activity (DEVD cleavage) within the sub ventricular zone, hippocampus and cerebellum of controll and asphyctic rats during the first 15 days after birth. The Caspase-3-like activity after global asphyxia was compared using a two-way ANOVA with a bonferroni correction for repeated measures. ${ }^{+} P=0.06$; ${ }^{*} P<0.05$. 
technique using antibodies against inactive and active Caspase-3 [25]. In present study, wo measured Caspase-3-like activity in different brain regions using a fluorometric DEVD cleavage assay. This method has been extensively used to study Caspase-3-like activity within cell cultures $[19,29]$ and homogenates of CNS tissue $[15,33]$. Furthermore, DEVD cleavage activity bas been shown to correlate positively with the activation of Caspase-3 [6]. The pattern of Caspase-3-like activity within the SVZ, hippocampus and cerebellum in both control and asphyctic rats showed a similar pattern of cell death as that described by others. This supports the idea that Caspase- 3 plays an important role in both physiological apoptosis and cell death after asphyxia with the early postnatal period [14,27].

Caspase-3 is a cysteine protease that is activated via cleavage of its proenzyme ( $32 \mathrm{kDa}$ protein) into smaller fragments $(20,17$ and $12 \mathrm{kDa})$ [10]. After activation Caspase-3 cleaves endogenous substrate proteins, such as the inhibitory protein of Caspase-activated DNase (ICAD) [12,37], poly(ADP) ribose polymerase (PARP) [11], lamins [34] and PAK2 (a member of the p21-activated kinase family) [36]. Inactivation or activation of these proteins by cleavage of caspase-3 leads to the morphological hallmarks of apoptosis, including the degradation of DNA into DNA fragments, nuclear shrinking and blebbing [17]. In our study, ultrastructural analysis revealed primarily apoptotic features within dying cells of asphyctic rats, which again supports the role of Caspase-3 in delayed cell death after global asphyxia at term.

Although our group and others described an increase in TUNEL-positive cell profiles within the striatum and cerebellum of asphyctic rats (global asphyxia at term) during the first postnatal week $[9,41]$, we did not observe an increase in Caspase-3-like activity within these regions after global asphyxia at term. This difference is likely due to both the selective cell loss within these regions after global asphyxia at term and the method of microdissection. Previously, we have shown that globall asphyxia at term only produced a very small amount of cell loss $(-16 \%)$ within the striatum [41]. In this study, the SVZ contains part of the cortex, striatum and the entire $S V Z$ region. There has not been any increase in cell death reported for the cortex within this global asphyxia model. In addition, cell death in the cerebellum after global asphyxia at term was only described for sparse cells [9]. Furthermore, we observed less Caspase-3 positive cell profiles than TUNEL-positive profiles in asphyctic rats, suggesting that the time window of Caspase-3 activity proceeds the time window of TUNEL-labelling.

\section{Global asphyxia at term versus meonatal hypoxia-ischemia}

Cell death after perinatal asphyxia has mainly been studied in rats using the Rice-Vannucci model [35], which induces cell death and infarction within the cerebral cortex, striatum, thalamus, hippocampus and in the white matter $[7,27,40]$. Both necrotic (cytoplasmic swelling, disruption of cell membrane, mitochondrial swelling, inflammation) and apoptotic (cellular shrinkage, nuclear chromatin condensation, nuclear fragmentation) features have been descibed in neurons and oligodendrocytes following this model $[27,28]$. We used a rat model that produces global asphyxia (rather than focal asphyxia) at term, which also induces delayed apoptotic neuronal and glial cell death within the striatum, hippocampus, cerebellum and spinal cord $[8,9,41]$. However, no clear necrotic features were visible within dying celis of asphyctic 
rats. Furthermore, previous studies showed that there is a lack of inflammation after global asphyxia at term $[9,42]$, supporting the idea that global asphyxia primarily induces delayed apoptotic cell death. The discrepancy in the severity and morphological type of cell death is likely due to the time of induction and type of insult. Global asphyxia is induced in a noninvasive matter reducing the blood and oxygen supply to the entire fetus at tcrm (P0) as occurs clinically during birth asphyxia. Neonatal hypoxia-ischemia is induced by unilateral carotid artery ligation at postnatal day $2,3,7,11$ or 21 followed by exposure to hypoxia ( $8 \%$ oxygen)

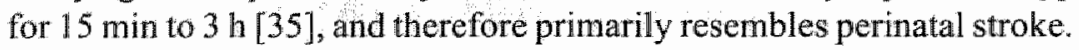

\section{Selective vulnerability of hippocampal neurons after global asphyxia at term}

Many studies have shown that the hippocampus is particularly susceptible to neonatal hypoxiaischemia and/or asphyxila. It is hypothesized that this is due to the large expression of NMDA and AMPA receptors within the hippocampus, which makes these cells more susceptible to excitotoxic cell death [31]. This is supported by previous studies showing attenuation of caspase3 activation after neonatal hypoxia-ischemia by administration of MK-801, a NMDA receptor antagonist [32]. Since, the hippocampus is primarily involved in spatial and object recognition (spatial memory) [26,30], loss of neurons and glial cells within this regions due to perinatal asphyxia may detrimentally affect cognitive performance in later life.

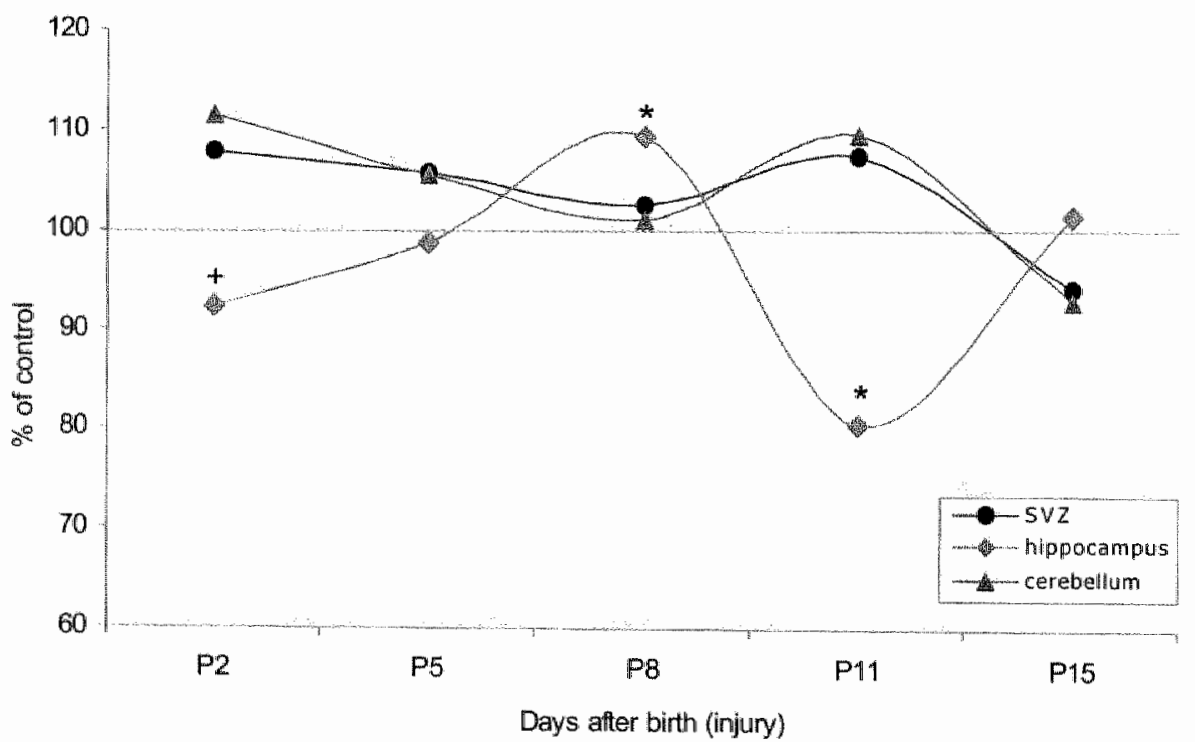

Figure 6. An overview of the effect of global asphyxia at term on Caspase-3-like acitvity during postnatal development. This graphs illustrates the increase in Caspase-3-like activity at $P 8$ and subsequently a drop in Caspase-3-like activity at $P 11$ within the hippocampus. * $P=0.06 ;{ }^{*} P<0.05$. 


\section{Conclusions}

In summary, global asphyxia at term primarily caused delayed apoptotic cell death within the proliferating areas of the striatum and hippocampus. Caspase-3-like activity was at high levels during the first week of life and declined afterwards in all areas examined. Global asphyxia caused an increase in caspase-3-like activity within the hippocampus at P8, followed by a drop in caspase-3-like activity. We conclude that this type of asphyctic insult (P0) causes hippocampal neuronal and glial death within the second week of life and without necrosis. This is in contrast to other models for neonatal hypoxia-ischemia (Rice-Vannucci) that induce necrotic cell death within hours after the insult and apoptotic cell death within 3 days after the injury [35].

\section{Acknowledgements}

We are grateful to Dr. Klas Blomgren and Dr. Henrik Hagberg for providing excellent advice concerning the DEVD cleavage assay. In addition, we would like acknowledge the expert technical assistance provided by R. van Gool for the ultrastructural analysis. 


\section{References}

[1] Back, S.A. Luo, N.L., Borenstein, N.S., Levine, J.M., Volpe, J.J. and Kinney, H.C. 2001. Late oligodendrocyte progenitors coincide with the develapmental window of vullnerability for human perinatal white matter injury, J Neurosci, $211302-12$

Back, S.A., Volpe, J.J. 1997. Cellular and molecular pathogenesis of periventricular white matter injury., Ment Retard Dev Disabil Res Rev, 396-107.

[3] Bayer, S.A., Altman, J., Russo, R.J and Zhang, X. 1993. Timetables of neurogenesis in the human brain based on experimentally determined patterns in the rat, Neurotoxicology, 14 83-144.

[4] Blaschke, A.J., Staley, K. and Chun, J. 1996. Widespread programmed cell death in proliferative and postmitotic regions of the fetal cerebral cortex, Development, 1221165 74.

[5] Blaschke, A.J., Weiner, J.A. and Chun, J. 1998. Programmed cell death is a universal feature of embryonic and postnatal neuroproliferative regions throughout the central nervous system, J Comp Neurol, 396 39-50.

[6] Blomgren, K., Zhu, C., Wang, X., Karlsson, J.O., Leverin, A.L., Bahr, B.A."Mallard, C. and Hagberg, H. 2001. Synergistic activation of caspase-3 by m-calpain after neonatal hypoxiaischemia: a mechanism of "pathological apoptosis"?, J Biol Chem, 276 10191-8.

[7] Bonfoco, E. Krainc, D., Ankarcrona, M., Nicotera, P. and Lipton, S.A. 1995. Apoptosis and necrosis: two distinct events induced respectively, by mild and intense insults with $\mathrm{N}$ methyl-D-aspartate or nitric oxide/superoxide in corticall cell cultures, Proc Natl Acad Sci US A, $927162-6$.

[B] de Louw, A.J., de Vente, J., Steinbusch, H.P., Gavilanes, A.W., Steinbusch, H.W., Blanco, C.E., Troost, J. and Vles, J.S. 2002. Apoptosis in the rat spinal cord during postnatal development; the effect of perinatal asphyxia on programmed cell death. Neuroscience, $112751-8$.

[9] Dell'Anina, E., Chen, Y., Engidawork, $E_{*}$, Andersson, K.; Lubec, G., Luthman, J. and HerreraMarschitz $Z_{1}$ M. 1997. Delayed neuronal death following perinatal asphyxia in rat, Exp Brain Res, 115 105-15.

[10] Du, Y, Bales, K.R., Dodel, A.C., Hamilton-Byrd, E., Horn, J.W., Czilli. D.L., Simmons, L.K., Ni, B. and Paul, S.M. 1997. Activation of a caspase 3-related cysteine protease is required for glutamate-mediated apoptosis of cultured cerebellar granule neurons, Proc Natl Acad Sci U S A, 94 11657-62.

[11] Eliasson, M.J., Sampei "K., Mandir, A.S., Hurn, P.D., Traystman, R.J., Bao, J., Pleper, A., Wang, Z.Q., Dawson, T.M. Snyder, S.H. and Dawson, V.L. 1997. Poly(ADP-ribose) polymerase gene disruption renders mice resistant to cerebral ischemia, Nat Med, 31089 95.

[12] Enari, M., Sakahira, H., Yokoyama, H., Okawa, K., Iwamatsu, A and Nagata, S. 1998. A caspase-activated DNase that degrades DNA during apoptosis, and its inhibitor ICAD, Nature, 391 43-50.

[13] Ferrer, 1., Tortosa, A., Blanco, R., Martin, F., Serrano, T., Planas, A. and Macaya, A. 1994. Naturally occurring cell death in the developing cerebral cortex of the rat. Evidence of apoptosis-associated internucleosomal DNA fragmentation, Neurosci Lett, 182 77-9.

[14] Fukuda, H., Tomimatsu, T., Watanabe, N., Mu, J.W., Kohzuki, M., Endo, M., Fujii, E., Kanzaki, T. and Murata, $Y$. 2001. Post-ischemic hypothermia blocks caspase-3 activation in the newborn rat brain after hypoxia-ischemia, Brain Res, $910187-91$.

[15] Gill, R. Soriano, M., Blomgren, K. Hagberg, H., Wybrecht, R., Miss, M.T., Hoefer, S. Adam, G., Niederhauser, O., Kemp, J.A. and Loetscher, H. 2002. Role of caspase-3 activation in 
cerebral ischemia-induced neurodegeneration in adult and neonatal brain, $\mathrm{J}$ Cereb Blood Flow Metab, 22 420-30.

[16] Gu, C., Casaccia-Bonnefil, P., Srinivasan, A. and Chao, M.V. 1999. Oligodendrocyte apoptosis mediated by caspase activation, J Neurosci, 19 3043-9.

[17] Hengartner, M.O. 2000. The biochemistry of apoptosis, Nature, 407 770-6.

[18] Katsetos, C.D., Spandou, E., Legido, A., Taylor, M.L., Zanelli, S.A., de Chadarevian, J.P., Christakos, S., Mishra, O.P. and Delivoria-Papadopoulos, M. 2001. Acute hypoxia-induced alterations of calbindin-D28k immunoreactivity in cerebellar Purkinje cells of the guinea pig fetus at term, I Neuropathol Exp Neurol, 60 470-82.

[19] Keramaris, E., Stefanis, L., MacLaurin, J., Harada, N., Takaku, K., Ishikawa, T., Taketo, M.M., Robertson, G.S., Nicholson, D.W., Slack, R.S. and Park, D.S. 2000. Involvement of caspase 3 in apoptotic death of cortical neurons evoked by DNA damage, Mol Cell Neurosci, $15368-79$.

[20] Kerr, J.F., Wyllie, A.H. and Currie, A.R. 1972. Apoptosis: a basic biological phenomenon with wide-ranging implications in tissue kinetics, $\mathrm{Br} J$ Cancer, 26 239-57.

[21] Legido, A., Parikh, N.A., Taylor, M.L., Kalleyias, J.K., de Chadarevian, J., Katsetos, C.D. 2002. Decreased immunoreactivity of Calbindin-D28K in cerebellar Purkinje cells of children with hypoxic/ischemic encephalopathy, Pediatric Academic Societies. Abstract nr. 2603.

[22] Loidl, C.F., Gavilanes, A.W., Van Dijk, E.H., Vreuls, W., Blokland, A., Vles, J.S., Steinbusch, H.W. and Blanco, C.E. 2000. Effects of hypothermia and gender on survival and behavior after perinatal asphyxia in rats, Physiol Behav, 68 263-9.

[23] Maciejewska, B., Lipowska, M., Kowianski, P., Domaradzka-Pytel, B. and Morys, J. 1998. Postnatal development of the rat striatum-a study using in situ DNA end labeling technique, Acta Neurobiol Exp (Warsz), $5823-8$.

[24] Milligan, C.E. and Schwartz, L.M. 1997. Programmed cell death during animal development, Br Med Bull, 53 570-90.

[25] Mooney, S.M. and Miller, M.W. 2000. Expression of bcl-2, bax, and caspase-3 in the brain of the developing rat, Brain Res Dev Brain Res, 123 103-17.

[26] Morris, R.G., Garrud, P., Rawlins, J.N. and O'Keefe, J. 1982. Place navigation impaired in rats with hippocampal lesions, Nature, $297681-3$.

[27] Nakajima, W., Ishida, A., Lange, M.S., Gabrielson, K.L., Wilson, M.A., Martin, L.J., Blue, M.E. and Johnston, M.V. 2000 . Apoptosis has a prolonged role in the neurodegeneration after hypoxic ischemia in the newborn rat, J Neurosci, $207994-8004$.

[28] Ness, J.K., Romanko, M.J., Rothstein "R.P., Wood, T.L. and Levison, S.W. 2001. Perinatal hypoxia-ischemia induces apoptotic and excitotoxic death of periventricular white matter oligodendrocyte progenitors, Dev Neurosci, $23203-8$.

[29] Oberdoerster, J. and Rabim, R.A. 1999. Enhanced caspase activity during ethanol-induced apoptosis in rat cerebellar granule cells, Eur J Pharmacol, 385 273-82.

[30] O'Keefe, J.a.N.L. 1978. The hippocampus as a cognitive map. Clarendon Press, Oxford.

[31] Portera-Cailliau, C. Price, D.L. and Martin, L.J. 1997. Excitotoxic neuronal death in the immature brain is an apoptosis-necrosis morphological continuum, J Comp Neurol, 378 70-87.

[32] Puka-Sundvall, M., Hallin, U., Zhu, C. Wang, X., Karlsson, J.O., Blomgren, K. and Hagberg, H. 2000. NMDA blockade attenuates caspase-3 activation and DNA fragmentation after neonatal hypoxia-ischemia, Neuroreport, $112833-6$.

[33] Puka-Sundvall, M., Wallin, C., Gilland, E., Hallin, U., Wang, X., Sandberg, M. , Karlsson, J., Blomgren, K. and Hagberg, H. 2000. Impairment of mitochondrial respiration after cerebral hypoxia-ischemia in immature rats: relationship to activation of caspase-3 and neuronal injury, Brain Res Dev Brain Res, 125 43-50. 
[34] Rao, L., Perez, D. and White, E. 1996. Lamin protealysis facilitates nuclear events during apoptosis, J Cell Biol, 135 1441-55.

[35] Rice, J.E., 3rd, Vannucci, R.C. and Brierley, J.B. 1981. The influence of immaturity on hypoxicischemic brain damage in the rat, Ann Neurol, $9131-41$.

[36] Rudel, T. and Bokoch, G.M. 1997. Membrane and morphological changes in apoptotic cells regulated by caspase-mediated activation of PAK2, Science, 276 1571-4.

[37] Sakahira, H. Enari, M. and Nagata, S. 1998. Cleavage of CAD inhibitor in CAD activation and DNA degradation during apoptosis, Nature, 391 96-9:

[38] Skoff, R.P., Bessert, D.A., Biarks, J.D., Song, D., Cerghet, M. and Silverstein, F.S. 2001. Hypoxic-ischemic injury results in acute disruption of myelin gene expression and death of oligodendroglial precursors in neonatal mice, Int J Dev Neurosci, 19 197-208.

[39] Song, H. Stevens, C.F. and Gage, F.H. 2002. Astroglia induce neurogenesis from adult noural stem cells, Nature, 417 39-44.

[40] Towfighi, J., Mauger, D., Vannucci, R.C. and Vannucci, S.d. 1997. Influence of age on the cerebral lesions in an immature rat model of cerebral hypoxia-ischemia: a light microscopic study, Brain Res Dev Brain Res, 100 149-60.

[41] Van de Berg, W.D., Schmitz, C., Steinbusch, H.W. and Blanco, C.E. 2002. Perinatal asphyxia induced neuronal loss by apoptosis in the neonatal rat striatum: a combined TUNEL and stereological study, Exp Neurol, 174 29-36.

[42] Van de Berg, W.D.J., Steinbusch, H.P.J, Veerbeek, L. Huiban, M." Lopez-Figueroa, M.O. De Vente, J., Blanco, C.E. Steinbusch, H.W.M. 2002. Direct imaging of nitric oxide production using DAF-2/DA after perinatal asphyxia in the rat striatum and cerebellum, Pediatric Academic Societies. Abstract nr. ??

[43] Volpe, J.J.2001. Perinatal brain injury: from pathogenesis to neuroprotection, Ment Retard Dev Disabil Res Rev, 7 56-64.

[44] Wagner, J.P., Black, I.B. and DiCicco-Bloom, E. 1999. Stimulation of neonatal and adult brain neurogenesis by subcutaneous injection of basic fibroblast growth factor, J Neurosci, $196006-16$.

[45] White, L.D. and Barone, S., Jr. 2001. Qualitative and quantitative estimates of apoptosis from birth to senescence in the rat brain, Cell Death Differ, 8 345-56.

[46] Williams, R.W. and Herrup, K. 1988. The control of neuron number, Aninu Rev Neurosci, 11 423-53. 


\section{Perinatal asphyxia increases DAF-2/NO fluorescence during postnatal rat development in the striatum, but not cerebellum}

W.D.J. Van de Berg, H.P.J. Steinbusch, L. Veerbeek, M. Huiban, M.O. Lopez-Figueroa, J. De Vente, C.E. Blanco, H.W.M. Steinbusch

European Journal of Neuroscience, in press 


\section{Abstract}

N-Methyl-D-aspartate receptor-mediated toxicity after perinatal asphyxia may depend on the generation of nitric oxide (NO). 4,5-diaminofluorescein (DAF-2) can react with $\mathrm{NO}$ in combination with $\mathrm{O} 2$ of $\mathrm{O} 2$ to yield the fluorescent derivative DAF-2 triazole. In the present study, we studied DAF-2/NO fluorescence following global asphyxia at term in slices of neonatal rat brain by confocal mieroscopy. Fluorescence in DAF-2 loaded slices was detected in cells and fibers in the striatum and cerebellum at postnatal day (P)5, P8 and $P 12$. Perinatal asphyxia led to a large increase in the intensity and density of DAF-2 fluorescent cells at P5 and P8 in the striatum, but not in the cerebellum. In consecutive slices, an increase of cGMP immunoreactivity was observed in the striatum and cerebellum at $\mathrm{P} 5$ and $\mathrm{P} 8$, whereas nNOS immunoreactivity was unchanged. There was no gliosis detected in the striatum or cerebellum during the first week after perinatal asphyxia. Incubation with $\mathrm{N}^{\mathrm{G}}$-nitro-L-arginine, a NOS inhibitor, suppressed the DAF-2 fluorescent signal in the striatum at all ages. Incubation with sodium nitroprusside, a NO donor, led to a strong increase of the fluorescent signal in both the striatum and the cerebellum. We conclude that perinatal asphyxia increases DAF-2/NO fluorescence and cGMP levels during the first week after birth within the striatum, but not within the cerebellum, without marked gliosis. 


\section{Introduction}

Perinatal asphyxia is a common cause of mortality and morbidity in childhood. The production of nitric oxide (NO) has been proposed as an important cause of neuronal death after asphyxia. NO is a free radical, which functions as an intercellular messenger molecule and is crucial for several signal transduction pathways used for memory formation, sensory processing, and the regulation of cerebral blood flow (for reviews see $[5,9,23]$ ). NO is synthesized by oxidation of L-arginine via NO synthases (NOS), yielding citrulline as a coproduct. NO reacts with the soluble is oform of the enzyme guanylyl cyclase, which leads to increased synthesis of the second messenger guanosine $3^{\prime}, 5^{\prime}$-cyclic monophosphate (cyclic GMP) [14,25]. In addition, NO can react with free radicals such as superoxide (O2) or molecular oxygen $(\mathrm{O} 2)$, which results in the formation of respectively peroxynitrite (ONOO/ONOOH) or dinitrogen trioxide (N2O3) [1,36].

Several researchers have studied NO production after perinatal asphyxia using indirect methods, such as measurement of NOS activity, nicotinamide adenine dinucleotide phosphate diaphorase (NADPH-d) presence or cGMP synthesis. Using a rat model in which asphyxia was induced intra-partum, Loidl et al. showed that perinatal asphyxia led to enhanced staining of NADPH-d in the striatum and cortex in newborn pups and adult rats (6 months of age), indicating increased expression of NOS. Furthermore, an increase in cyclic GMP (cGMP) levels was detected in this model in the striatum 10 days after perinatal asphyxia [20]. However, total NOS activity was not increased after perinatal asphyxia in this model and other models inducing hypoxia-ischemia during development $[10,24]$.

Recently, a fluorometric method has been introduced for imaging of intracellular NO based on the utilization of the cell permeable 4,5-diaminofluorescein-2 diacetate (DAF-2/DA). DAF-2/DA penetrates the cell membrane and is hydrolyzed by cytosolic esterases, producing. DAF-2. DAF-2 reacts with NO to form the highly fluorescent derivative DAF-2 triazole $[12,16,17,26]$. DAF-2 has high selectivity for NO [16]. Furthermore, the DAF-2 has a low detection limit (2-5 nM) and has been used for evaluation of intracellular NO in cell cultures and slices $[2,21,22]$. However, recent studies have shown that DAF- 2 is also able to react with peroxynitrite [29], and dehydroascorbic acid (DHA) and ascorbic acid (AA) $[13,37]$. The selectivity of DAF-2 in different biological systems under both physiological and pathophysiological conditions are still unclear. The actual fluorescent product is probably a nitrosylated product of DAF-2, which we abbreviate as DAF-2/NO.

In the present study, we investigated whether perinatal asphyxia increases DAF-2/NO fluorescence within the striatum and cerebellum during postnatal development. The results of the DAF-2/DA method were compared with results of immunohistochemistry for $\mathrm{nNOS}$ and cGMP of the same tissue. We hypothesized that perinatal asphyxia has long-lasting effects of the NO synthesis in both the striatum and cerebellum. 


\section{Materials and methods}

\section{Animals}

Twelve full-term pregnant Wistar rats and their male pups $(n=48)$ were used (Charles RiverBroekmans, Someren. The Netherlands). They were housed under standard conditions (12:12 h light:dark cycles, $20^{\circ} \mathrm{C}$ ) with free access to standard laboratory chow and water. The local Committee on Animal Welfare approved all animal care and procedures.

\section{Induction of perinatal asphyxia}

Global asphyxia was induced in rat pups at P0 by placing the uteri and its contents in a water bath for 20 minutes, as described previously [32]. Briefly, time-pregnant Wistar dams were decapitated immediately after delivery of two pups (CVD) and rapidly hysterectomized. The uterus horns containing the remaining pups were placed in a water bath at $37^{\circ} \mathrm{C}$ for $20 \mathrm{~min}$ (severe perinatal asphyxia; SPA). Afterwards, pups were removed from the uterus homs and stimulated to breath by cleaning their skin and by gently padding them on the chest. The maximal duration of the resuscitation was 5 minutes. If a pup was not able to gasp, it was excluded from the study. The pups were left to recover for 60 min in a paediatric incubator at $36.5^{\circ} \mathrm{C}$ and afterwards randomly assigned to a surrogate mother ( 10 pups per mother), which had given birth on the same day. The percentage of mortality in the SPA and CVD group was respectively $\pm 50 \%$ and $0 \%$.

\section{4,5-Diaminofluorescein diacetate (DAF-2/DA) detection in slices}

DAF-2/NO fluorescence was studied in slices $(300 \mu \mathrm{m})$ of rat pups at postnatal (P)5, P8 and P1.2 ( $=6$ per group at each time point) using DAF2/DA detection assay (Sigma, The Netherlands). Rat pups were decapitated and their brains were rapidly removed and placed into ice-cold Krebs-Ringer bicarbonate buffer ( $\mathrm{pH} 7.4$ ) areated with $95 \% \mathrm{O} 2 / 5 \% \mathrm{CO}$. The forebrain and the cerebellum were chopped into $300 \mu \mathrm{m}$ coronal slices using a Mclllwain tissue chopper. Slices were separated under a microscope and transferred to multiwell culture plate containing Krebsbuffer $\left(4^{\circ} \mathrm{C} ; \mathrm{pH} 7.4\right)$ and $1 \mathrm{mM}$ isobutyl-methyl xanthine (IBMX) to inhibit 3',5'-cyclic nucleotide phosphodiesterase (PDE) activity. Alternated slices were transferred to a second multiwell plate and used for immunohistochemistry.

All slices were incubated in Krebs-Ringer buffer containing IBMX for 30 min and slowly warmed to $35.5^{\circ} \mathrm{C}$ or $25.5^{\circ} \mathrm{C}$ under an atmosphere of $5 \% \mathrm{CO} 2 / 95 \% \mathrm{O} 2$. Slices were incubated in the presence or absence of the NO donor $0.1 \mathrm{mM}$ sodium nitroprusside (SNP; Sigma) for $10 \mathrm{~min}$, or the NOS inhibitor $0.1 \mathrm{mM} \mathrm{N} \mathrm{N}^{\mathrm{a}}$-nitro-L-arginine (Sigma) for $30 \mathrm{~min}$. $\mathrm{N}^{\mathrm{G}}-\mathrm{L}$-nitroarginine was included in the experimental solution throughout the remainder of the experiment. The slices were incubated with $1.5 \mathrm{ml} 10 \mathrm{mM}$ DAF-2/DA solution (150mM Tris-HCl, $3 \mathrm{mM}$. tetrahydrobiopterin, $1 \mathrm{mM}$ thavin adenine dinucleotide (FAD, Sigma), $1 \mathrm{mM}$ flavin mononucleotide (FMN, Sigma), $1 \mathrm{mMNADPH}, 0.6 \mathrm{mM} \mathrm{CaCl}$ (Merck) and $100 \mathrm{mM}$ L-arginine 
(Sigma) in distilled water, $\mathrm{pH} 7.4$ ) per well for 45 min at $35.5^{\circ} \mathrm{C}$ or $25.5^{\circ} \mathrm{C}$. During incubation with the DAF-2/DA solution, lights were turned off and a dark box was placed over the incubation chamber. As a negative control, slices were incubated in media lacking DAF-2/DA. Following incubation with DAF-2/DA, the slices were washed in phosphate-buffered saline (pH 7.4) and placed on non-coated slides using a $5 \mathrm{ml}$ pipet with a cut tip (without touching the slices) and coverslipped using PBS-glycerol (1:4).

The DAF-2/NO fluorescent signal was measured immediately after the experiment. Digital images were captured using a CoolView CCD camera system attached to a MR C600 confocal microscopy (Leica Microsystems, Germany). The microscope was equipped with an excitation ( $488 \mathrm{~nm}$ ) and emission $(530 \mathrm{~nm})$ green filter for fluorescein. Images were taken at different levels ( $10 \mu \mathrm{m}$ vertical distance) throughout the slice, yielding approximately 25 images per area per slice, using the same settings throughout the whole experiment. The images were combined into one image per area using image analysis software (Biorad). From each rat pup, 3 slices per area (striatum and cerebellum) were analyzed using the semi-quantitative histogram analysis of the images (grey values) using the SIS image analysis system (AnalySIS ${ }^{\mathrm{v}}$, Germany).

\section{4,5-Diaminofluorescein diacetate (DAF-2/DA) detection in frozen sections}

The DAF-2/DA staining on frozen tissue was performed according to the method of LopezFigueroa et al. [22]. CVD $(n=3)$ and SPA $(n=3)$ rat pups were decapitated at P5 and P8 and $P 10$ and their brains were rapidly removed and snap frozen using liquid nitrogen. The forebrain was cut to serial, $16 \mu \mathrm{m}$ thick, coronal sections on a cryostat. The sections were washed in $25 \mathrm{mM}$ Tris- $\mathrm{HCl}(\mathrm{pH} \mathrm{7.4)}$ for $5 \mathrm{~min}$ at room temperature, washed twice in TBS (pH 7.4) for 5 min at $37^{\circ} \mathrm{C}$. This was followed by incubation in $10 \mathrm{mM} \mathrm{DAF-2/DA}$ (Sigma) incubation buffer for $90 \mathrm{~min}$ at $37^{\circ} \mathrm{C}$. After $45 \mathrm{~min}$ incubation, buffer was refreshed once. The sections were washed once with TBS at room temperature, and coverslipped using TBS:glycerol (1:4). The optimal incubation time was tested on several sections of different animals. The best result was seen after $90 \mathrm{~min}$ incubation time (refreshing the incubation buffer after $45 \mathrm{~min}$ ) using a DAF-2/DA concentration of $10 \mathrm{mM}$.

Control sections were preincubated in $0.1 \mathrm{mM} \mathrm{N}^{\mathrm{C}}$-nitro-L-arginine for $10 \mathrm{~min}$, washed twice in TBS at $37^{\circ} \mathrm{C}$ and incubated in DAF-2/DA buffer containing $0.1 \mathrm{mM} \mathrm{N}{ }^{\mathrm{G}}$-nitro-L-arginine for $90 \mathrm{~min}$ at $37^{\circ} \mathrm{C}$. To detect background levels, sections were incubated for $90 \mathrm{~min}$ at $37^{\circ} \mathrm{C}$ with the incubation buffer lacking DAF-2/DA. To investigate whether fixation would improve DAF-2/NO fluorescent signal, we fixed sections with 4\% paraformaldehyde in TBS ( $\mathrm{pH} 7.4$ ) for $10 \mathrm{~min}$ prior to incubation with the DAF-2/DA solution. All sections were examined at a magnification of $\times 200$ with an Olympus AX-70 microscope, using epifluorescent illumination. Pictures were captured using a CCD video camera. 


\section{Immunohistochemistry}

Adjacent slices were used to visualize cGMP-producing and NOS active structures, using antisera against formaldehyde-fixed CGMP [7] and nNOS [11]. Furthermore, we used antisera against glial fibrillary acidic protein (GFAP; Sigma) or vimentin (Zymed) as glial markers. After incubation in the presence or absence of SNP or $N^{G}$-nitro-L-arginine, slices were fixed for $2 \mathrm{~h}$ with ice-cold $4 \%$ (wt/vol) freshly made paraformaldehyde in $0.1 \mathrm{M}$ phosphate buffer $\mathrm{pH} 7.4)$, containing $10 \%$ sucrose. Slices were aligned in a plane, quickly frozen into TissueTek OCT compound using $\mathrm{CO} 2$ and $10 \mu \mathrm{m}$ thick sections were cut on a cryostat. Sections were stored at $-20^{\circ} \mathrm{C}$ until further processing.

All sections were washed and rinsed with TBS and TBS with $0.3 \%$ Triton X-100 (TBS-T). The sheep anti-cGMP and anti-nNOS antisera were used respectively at a dilution of 1:4,000 and 1:2,000 and visualized using a Alexa-conjugated donkey anti-sheep antibody (1:100; Mol. Probes). Mouse monoclonal antibodies against glial fibrillary acidic protein (GFAP) and vimentin were used respectively at a dilution of $1: 1,600$ and $1: 2$ and visualized with a Cy3conjugated donkey anti-mouse antiserum (1:800). The sections were incubated overnight at $4^{\circ} \mathrm{C}$ with the primary antibody and $2 \mathrm{~h}$ at room temperature with the secondary antibody. Parvalbumin was visualized using a rabbit antiserum $(1: 1,500)$ kindly provided by P.C. Emson (Babraham Institute, Cambridge, UK) in combination with a donkey anti-rabbit biotinylated antiserum $(1: 400)$ and streptavidine-Cy $3(1: 2,000)$.

Images of the sections were captured using CCD camera attached to an Olympus AX-70 microscope equipped with a narrowband MNIBA-type FITC filter, or MNG filter for CY fluorescence (Chroma technology). All images were saved on computer files and analyzed using the analySIS ${ }^{3}$ system. All sections were stained simultaneously and recorded on the same day to minimise experimental variation.

\section{Image analysis}

Fluorescence intensity of the images obtained fron the slices loaded with DAF-2/DA and immunostained sections (cGMP, nNOS) were analyzed using a macro designed for measuring grey values in a given area (region of interest, ROI). Background values were measured using the slices incubated with the incubation buffer lacking the DAF-2/DA or lacking the primary antibodly. Measurements were performed on 3 sampled images of 3 systernatically sampled slices. From these data, the mean grey value in the area (striatum or cerebellum) was calculated for each area.

GFAP and vimentin immunofluorescence intensity in the striatum and cerebellum was converted into grey values divided in 16 classes. Differences between control and asphyctic rats in the amount of pixels per grey value intensity class were analyzed for the 7 classes with the maximum pixel number. Measurements were performed on 3 sections per slice ( 3 slices per animals). 


\section{Statistical analysis}

Data are expressed as mean tstandardard error of means ( \pm S.E.M). Grey values of immunofluorescence intensity measurements were evaluated using a Student's t-test. The level of significance was $P<0.05$ for all analyses.

\section{Results}

\section{The distribution of DAF-2 in slices (ex vivo)}

\section{$D A F-2$ distribution and intensity in control rats}

Slices of the striatum and cerebellum of control and asplyyctic rats (P5, P8 and P12) were examined using a confocal microscope after loading with DAF-2/DA. A bright fluorescent signal could be observed in the striatum and cerebellum of all pups. The fluorescent signal was localized intracellular in neuron-like cells, proximal dendrites and vascular structures (Figure 1). The fluorescent signal was punctate and seemed to be confined to the cytoplasm of the neuron (only visible at high magnification).

The lifetime of the fluorescent signal was determined by the duration and intensity of illumination. No direct fading or loss of intensity was seen when studying the slices under the confocal laser scanning microscope. After $5 \mathrm{~h}$ (slices were stored in between in a cold chamber at $4^{\circ} \mathrm{C}$ ) the fluorescent signal became more diffuse and cells were more difficult to distinguish. After $24 \mathrm{~h}$, the fluorescent signal had faded and had a diffuse appearance. Similar observations were made in cortical slices (data not shown).

\section{The effect of perinatal asphyxia on DAF-2 distribution and intensity}

Fluorescence in DAF-2 loaded slices of control rat pups was clearly visible throughout the entire striatum, predominantly in the dorso-medial part of the striatum. The intensity of the fluorescent signal in the striatum was higher at P5 and P8 than at P12 in control rat pups (P = 0.04). The intensity of the fluorescent signal was increased in slices of perinatal asphyctic rat pups at $\mathrm{P} 5$ and $\mathrm{P} 8$ compared to the control rats $(\mathrm{P}=0.04)$, but not at $\mathrm{P} 12(\mathrm{P}>0.05)$. Within the cerebellum, DAF-2/NO fluorescence was visible throughout the molecular and granular cell layer. There was no apparent difference in fluorescence pattern or intensity in the cerebellum between control and asphyctic rats at P5, P8 or P12 (see Figure 1). The fluorescent signal was however less intense at $\mathrm{P} 12$ than at $\mathrm{P} 5$ or $\mathrm{P} 8(\mathrm{P}=0.04)$.

The effect of temperature and NO modulators on DAF-2 distribution and intensity The effect of temperature on the DAF-2/NO fluorescent signal in slices was tested by incubation of the slices at $25.5^{\circ} \mathrm{C}$ instead of $35.5^{\circ} \mathrm{C}$. The lower temperature helped to preserve the quality 
of the slices, but led to a major decrease in the brightness of the fluorescent signal in both the striatum and cerebellum (Figure 2). Incubation with $0.1 \mathrm{mM} \mathrm{N}^{\mathrm{G}}$-nitro-L-arginine slightly suppressed the DAF-2/NO fluorescent signal at $\mathrm{P} 5, \mathrm{P} 8$ and $\mathrm{P} 12$ in both striatum and cerebellum. Pre-incubation with the NO donor SNP led to a major increase in the intensity and density of the fluorescent signal in the both striatum and cerebellum. Background levels were more intense after pre-incubation with SNP and almost all cells showed an extremely bright diffuse fluorescence (see Figure 2).
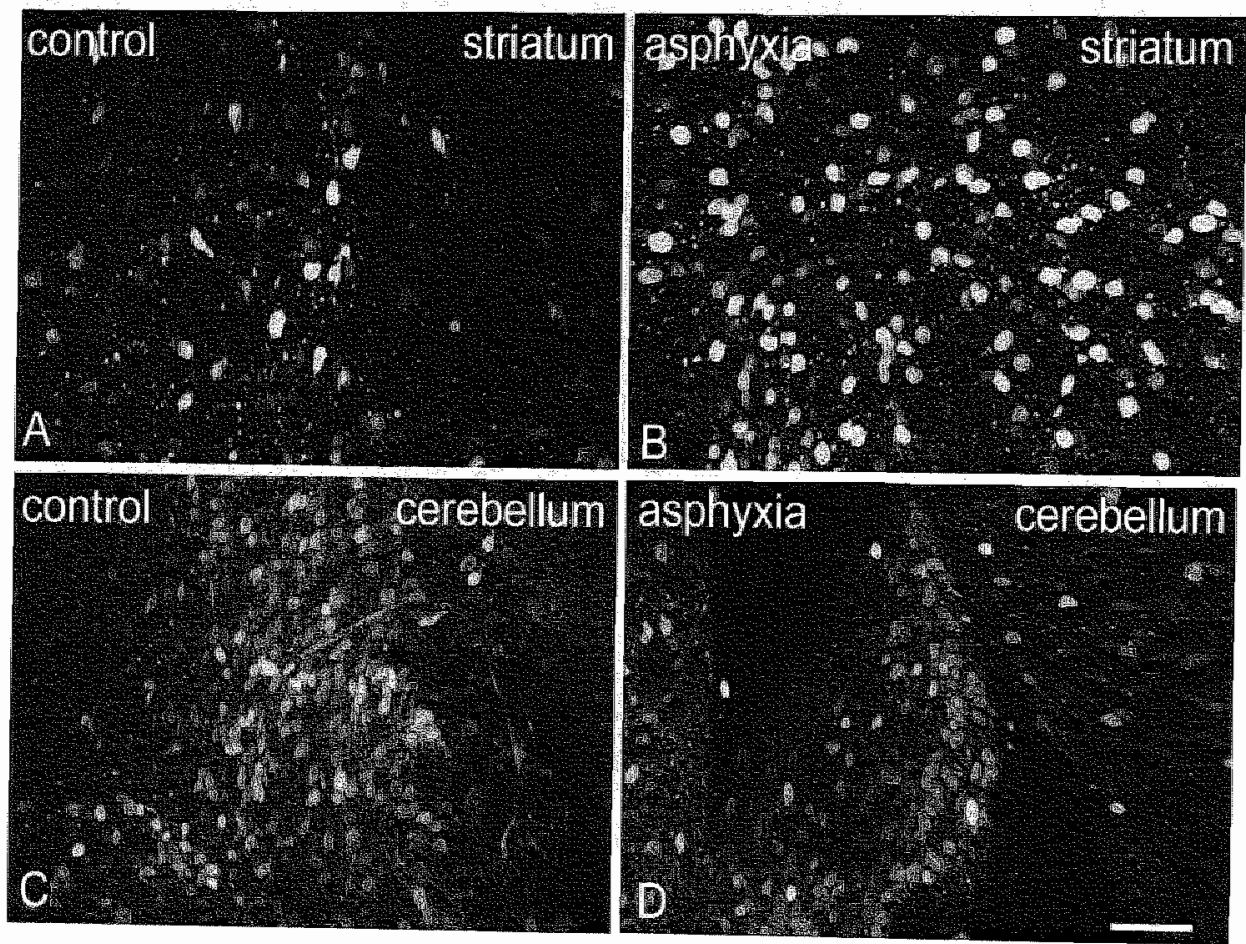

Figure 1. DAF-2 fluorescence in tissue slices from striatum $(A, B)$ and cerebellum (C,D) of a control $(A, C)$ and asphyctic rat $(B, D)$ at postnatal day 8 . Images were taken throughout the slice $(300 \mu \mathrm{m})$ with a confocal laser scanning microscope, and combined into one image per area using an image analysis system. Scale bar is $50 \mu \mathrm{m}$ for all photographs. 

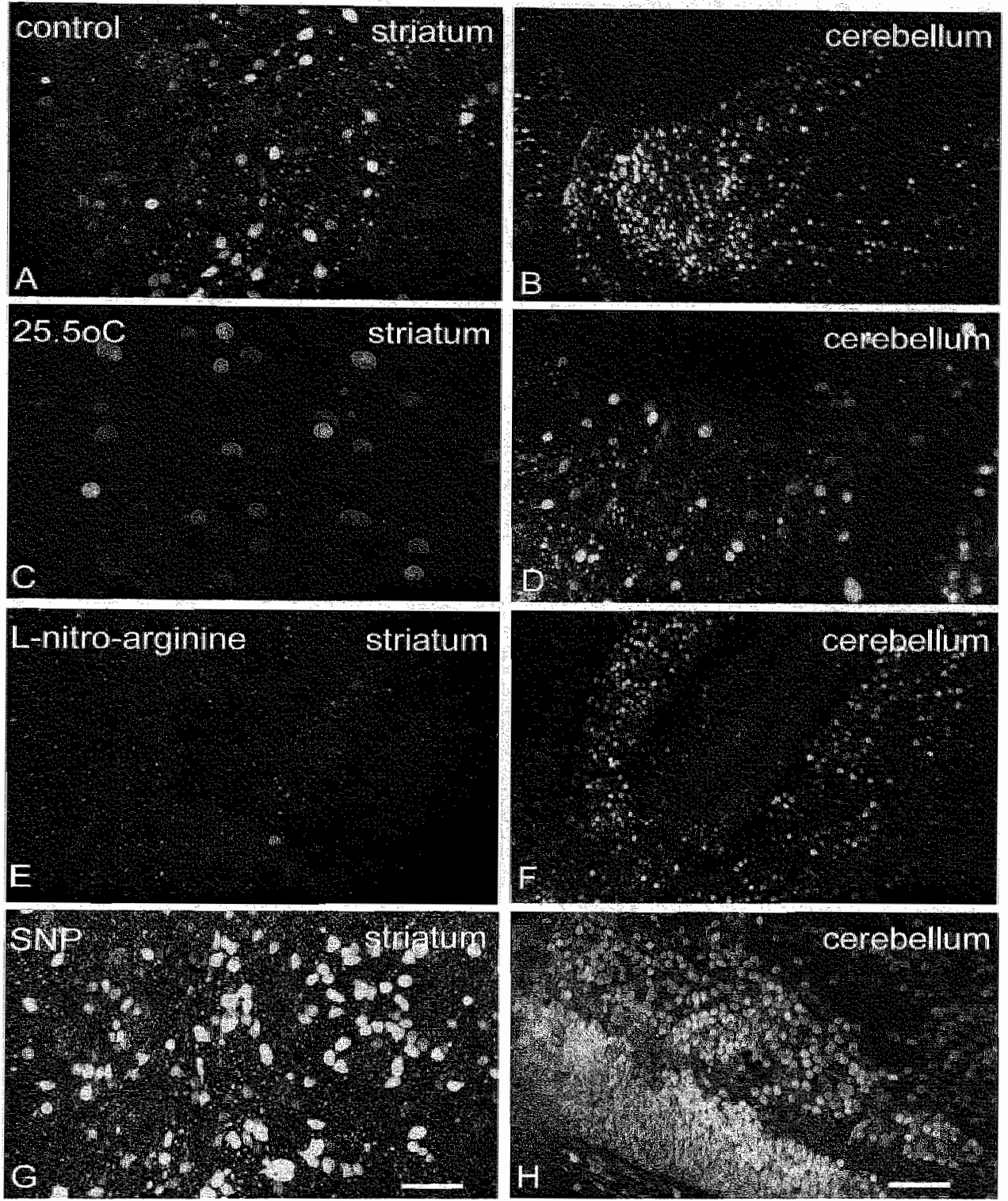

Figure 2. DAF-2 fluorescence in tissue slices of a control rat incubated $(A, B)$ in the presence off $1 \mathrm{mM} I B M X$ at $35.5^{\circ} \mathrm{C}$ for $45 \mathrm{~min}$; and $(\mathrm{C}, \mathrm{D})$ in the presence of $1 \mathrm{mM} I \mathrm{BMX}$ at $25.5^{\circ} \mathrm{C}$ for $45 \mathrm{~min} ;\left(E_{y} F\right)$ incubated in the presence of $0.1 \mathrm{mM} \mathrm{N}$-nitro-L-arginine at $35.5^{\circ} \mathrm{C}$ for $45 \mathrm{~min}$; and $(G, H)$ incubated in the presence of $0.1 \mathrm{mM} \mathrm{SNP}$ at $35.5^{\circ} \mathrm{C}$ for $45 \mathrm{~min}$. Hypothermia or incubation with $N^{G}$-nitro- $L$-arginine led to a decrease in fluorescent signal in both the striatum $(C, E)$ and cerebellum (D, F). Pre-incubation with SNP led to a strong increase of the fluorescent signal in both the striatum (G) and cerebellum (H). Scale bar is $50 \mu \mathrm{m}$ for all photographs. 


\section{DAF-2/DA system on frozen tissue}

DAF-2/NO fluorescence was observed in neuron-like cells of the striatum and cerebellum in both control and asphyctic rat pups at all ages. The fluorescent signal was observed extracellular and intracellular and showed a fine punctate pattern with a morphological appearance that was not apparently different between rats or groups (Figure 3 ). There was no difference in the intensity of the fluorescent signal between sections pre-incubated with or without SNP or $N^{G_{\alpha}}$ nitro-L-arginine. There was no fluorescent signal in sections incubated with DAF-2/DA buffer lacking $\mathrm{CaCl} 2$ or L-arginine, or incubation buffer lacking DAF-2/DA. Fixation of the section prior to DAF-2/DA loading did not improve the fluorescent signal, but led to a major decrease in intensity of the fluorescent signal. Continuous exposure of the section to the fluorescent light led to a rapid fading and loss of fluorescent signal (over a period of $5 \mathrm{~min}$ ).

There were no differences in intensity or density of the fluorescent signal between control or asphyctic rats at P5 or P8 in cryostat section ( $\mathrm{P}>0.05)$, contrary to what was observed in slices. Moreover, there were no differences in intensity or density of the signal between ages ( $\mathrm{P} 5$ versus $\mathrm{P} 8 ; \mathrm{P}=0.05)$ ). The fluorescent signal was more intense in the cerebellum than in the striatum at all ages in all animals $(P=0.04)$.
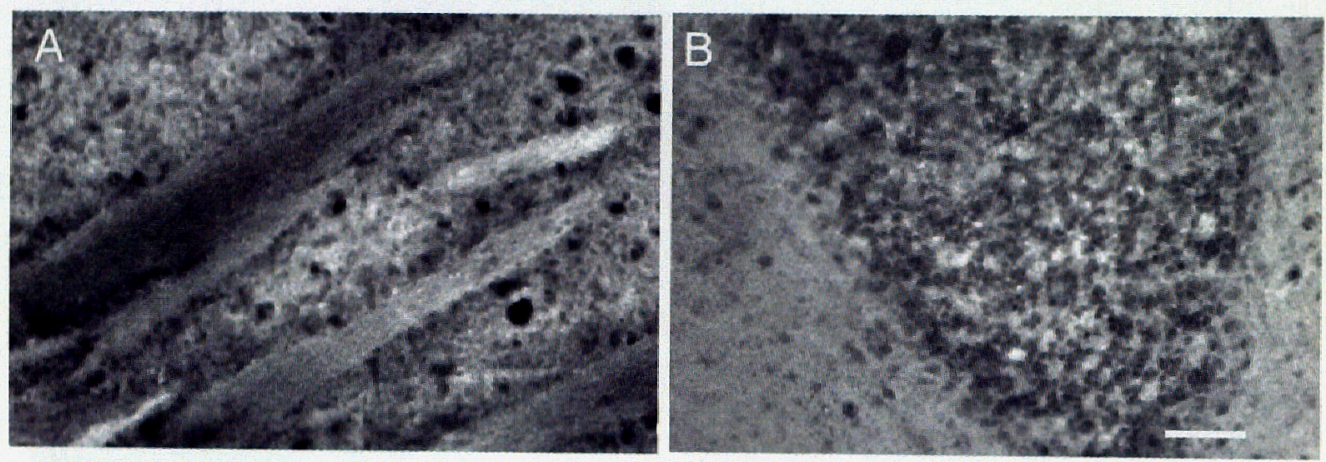

Figure 3. Photographs of fresh frozen sections $(16 \mu \mathrm{m})$ incubated with $\mathrm{DAF}-2 / \mathrm{DA}$ at $37^{\circ} \mathrm{C}$ for 90 min of a 8 day old control rat of A) striatum and B) cerebellum. Fluorescent signal was observed extracellular and intracellular and showed a fine punctate pattern. Scale bar is $20 \mu \mathrm{m}$.

\section{Immunohistochemistry}

Data on NO-mediated cGMP synthesis were obtained from slices incubated with $1 \mathrm{mM}$ IBMX to inhibit PDE activity. Incubation of the rat slices at P5, P8 and P12 with $1 \mathrm{mM} I B M X$ resulted in intense cGMP immunoreactivity (cGMP-IR) in cells and fibers in the striatum and cerebellum. Pre-incubation with $0.1 \mathrm{mM} \mathrm{NG}$-L-nitro-arginine abolished the cGMP signal, indicating that this NOS inhibitor abolished NOS activity and as a consequence NO production and cGMP synthesis. Incubation of the slices with SNP $(0.1 \mathrm{mM})$ resulted in an increase of 
cGMP-IR in cells and fibers in both the striatum and cerebellum. nNOS-IR was observed in cell bodies and varicose fibers in grey and white matter of striatum and cerebellum. Colocalization between cGMP and parvalbumin was found in cells and fibers in the striatum and cerebellum at P5, P8 and P12. There was no co-localization visible between nNOS and parvalbumin (see Figure 4). An increase of cGMP-IR was found in cells and fibers throughout the striatum of asphyctic rats compared to control rat at P5 and P8 $(\mathrm{P}<0.05)$, but not at P12 $(\mathrm{P}>0.05)$. There was no detectable difference in $\mathrm{nNOS}-\mathrm{IR}$ between control and asphyctic rats in the striatum or cerebellum $(\mathrm{P}>0.05)$.

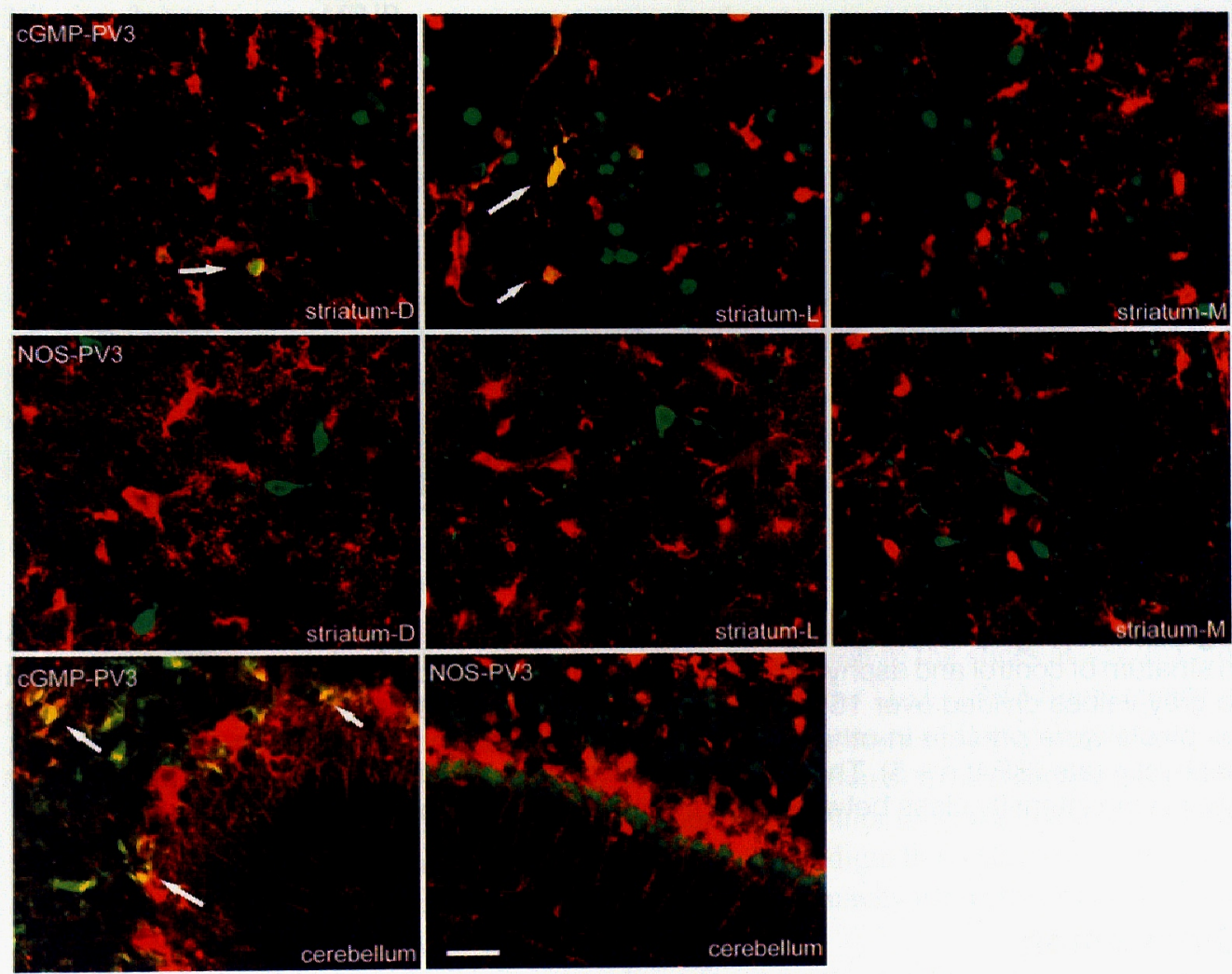

Figure 4. Double labeling of cGMP and parvalbumin (PV-3) in dorsal (A), lateral (B) and medial striatum (C) and cerebellum (G). Arrows indicate double labeled cells or fibers. Double labeling of $n N O S$ and $P V-3$ in dorsal $(E)$, lateral $(F)$ and medial $(G)$ striatum and cerebellum $(H)$. There was co-localization found between cGMP and PV-3, but not between nNOS and PV-3. Scale bar is $50 \mu \mathrm{m}$. 
GFAP-IR was very weak or not visible at P5. At P8 and P12, GFAP-IR was weak in the grey matter, but strong in the white matter. Vimentin-IR was clearly wisible in striatum and cerebellum in both white and grey matter at P5, P8 and P12 in control and asphyctic rats. The results of the image analysis in the striatum and cerebellum did not reveal any differences between control and asphyctic rats at any time point (see Figure 5; $\mathrm{P}>0.05$ ). The histogram of the grey value distribution was determined of 3 sections sampled throughout the obtained slices. The fluorescence pattern appeared to be relatively homogeneous over the entire slice in this experiments as judged by comparison between the section position and the amount of pixels in each class.

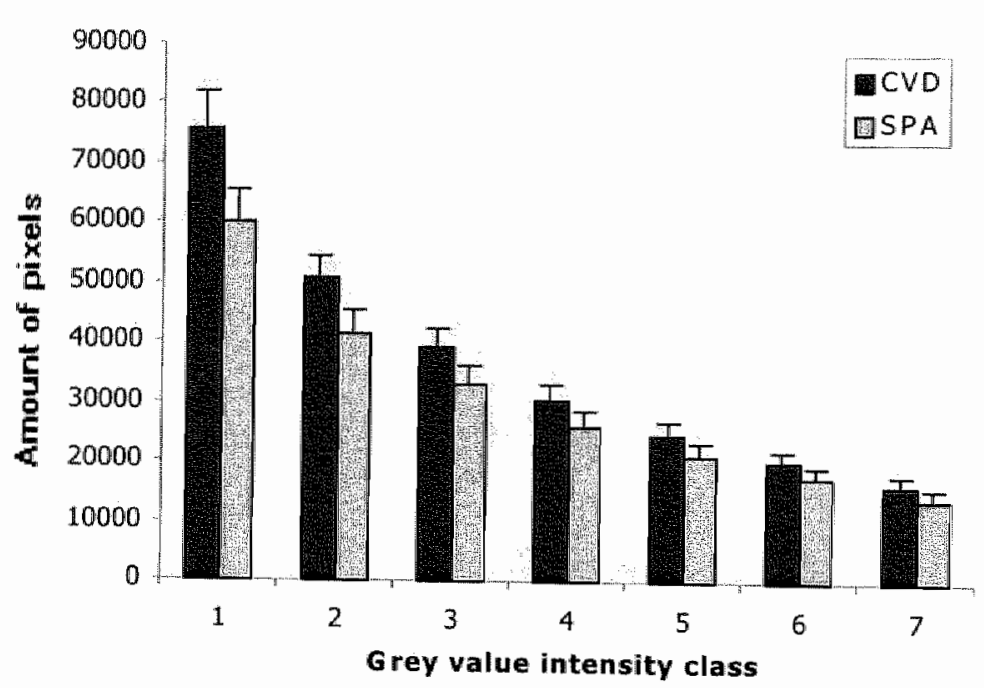

Figure 5. Histogram of division of pixels over intensity classes of GFAP immunoreactivity in striatum of control and asphyctic rats, at PB. Immunofluorescence intensity was converted to grey values divided over 16 classes. The results of classes 10-16 are shown as almost no pixels were present in other classes. Black bar: control rats (CVD; $n=5$ ); Grey bar: asphyctic rats (SPA; $n=5$ ). There was no difference in distribution or amount of pixels per grey value intensity class between the groups. Data are expressed as mean $\pm S$.E.M.

\section{Discussion}

In the present study, we provided evidence for an increase in DAF-2/NO fluorescence after perinatal asphyxia within the striatum at 5 and 8 days, but not in the cerebellum, during the first 8 days of life. DAF- $2 / \mathrm{NO}$ fluorescence in slices was observed in the cytoplasm and dendrites of a subpopulation of neurons in the striatum and cerebellum. Furthermore, cGMPIR within the same slices was increased after the perinatal asphyxia at P5 and P8, whereas 
NOS-IR was unchanged. Both GFAP and vimentin-IR were unchanged after perinatal asphyxia, indicating that there was no gliosis induced by the injury. These data show that perinatal asphyxia induces an increase in NO synthesis up to 8 days after the injury within the striatum. Since NOS-IR is unchanged by the asphyctic insult, we speculate that glutamatergic activation of NOS is increased.

To confirm that the DAF-2 has a high selectivity for NO, we studied the effect of a NO modulators (NOS inhibitor and NO donor) on the cellular distribution and intensity of the fluorescent signal. DAF-2/NO fluorescence was attenuated in slices incubated with $\mathrm{N}^{\circ}$-nitroL-arginine (NOS inhibitor) and intensified with SNP (NO donor). Previously, Brown et al. described an inhibition of DAF-2/NO fluorescence with the NOS inlibitor L-NAME in slices of adult rats [2]. Taken together, these data show the the DAF-2/NO fluorescent signal is dependent on NOS activity and NO production within the slice and can easily be modulated by $\mathrm{N}^{\mathrm{O}}$-nitro-L-arginine and SNP.

Kojima and co-workers demonstrated that DAF-2 has a high selectivity for $\mathrm{NO}$ [16]. Furthermore, they showed that DAF-2 could provide reliable information on intracellular NO levels regardless of the $\mathrm{Ca}^{2+}$ and $\mathrm{Mg}^{2+}$ concentrations [31]. The selectivity and detection limit of DAF-2 NO fluorescence make it a suitable method for detecting intracellular NO in living cells or slices. Furthermore, the location of DAF-2/NO fluorescent cells in slices correlated well with the distribution of NOS as described in immunohistochemical studies or by staining with NADPH-dependent diaphorase [2]. Our study provides further evidence for the suitability of the DAF-2 system for visualization of NO presence within neurons. We show that DAF-2/ NO fluorescence is easily detected in slices using confocial microscopy. Furthermore, we show that results obtained using the DAF-2/DA assay in slices correlate well with data obtained using CGMP-IR measurement in the same slices.

Our data also show that DAF-2/NO fluorescence is affected by the cell conditions, fixation, concentration and/or time of incubation. For example, the DAF-2 fluorescent signal appeared punctate (extra- and intracellular) and weak in the fresh frozen sections, compared to the strong intracellular signal in the slices. The punculated distribution may indicate that DAF 2 is incorporated into vesicles in the cells. Furthemore, incubation of the slices at $25-26^{\circ} \mathrm{C}$ for $45 \mathrm{~min}$ led to a major decrease of intensity and density of the DAF-2/NO flurorescence in cells and fibers compared to incubation at $35.5^{\circ} \mathrm{C}$. An earlier study of Lopez-Figueroa et $a$. showed that the DAF-2 system was able to detect NO production in fresh frozen tissuc of rats injected with IL-1 b. In this model for CNS inflammation a major increase in inducible NOS (iNOS) mRNA and protein was observed. The asphyxia paradigm that was used in our study, does not lead to a major inflammation [8], which might explain why we were not able to detect any changes in the frozen sections after perinatal asphyxia.

Several researchers have provided indirect evidence for an increase or change in NO production in the striatum in the same asphyxia paradigm. An increase in NADPH diaphorase histochemical staining, an indicator of NOS-containing cells [34], was found in striatal cells in newborn pups and 6 months old rats exposed to perinatal asphyxia [19]. Furthermore, an increase in cGMP levels in the striatum were observed 10 days after the induction of perinatal asphyxia, suggesting NOS activity and NO production was increased [20]. At the protein level, an increase 
in immunoreactive nNOS $136,000 \mathrm{~mol}$ wt protein using Westem blot analysis was described. At the transcriptional and activity level of NOS, no changes were found in whole brain samples using northerin and dot blot analysis, and NOS activity assay [24]. Taken together with our data, these results suggest that this perinatal asphyxia paradigm has allong-lasting effect on the NO synthesis within the striatum resulting in an increase in cGMP.

In an earlier study, we showed that perinatal asphyxia predisposes cells in the striatum to die especially in the first 8 days after the insult [33]. Moreover, Dell 'Anna ef al, showed that cell damage occurred mainly in the striatum, frontal cortex and cerebellum at P8 in this model of perinatal asphyxia [8]. An increase in reactive oxygen species was detected in the striatum of perinatal asphyctic rats after reoxygenation in the striatum, indicative of a strong involvement of free radicals in cellular damage after the injury [4]. Our data show that asphyxia triggers a long-lasting reaction leading to an increase in NO synthesis up to 8 days after the injury. Taken together, this suggests that NO might play an important role in mediating cellular damage in the striatum after perinatal asphyxia. Indeed, NOS inhibitors are potent neuroprotective agents in most animals model for hypoxia-ischemia and excitotoxicity in vitro $[3,10,27,30]$ NO is produced in nNOS-containing neurons, localized all throughout the brain, after activation of glutamatergic receptors. In the striatum, virtually all $\mathrm{NNOS-containing} \mathrm{neurons} \mathrm{are} \mathrm{GABA-}$ ergic and contain neuropeptide $Y$ and somatostatin [18,35]. cGMP immunoreactivity is present in cholinergic and parvalbumin-containing neurons in the striatum [6]. Indeed, in the present study, co-localization of cGMP and parvalbumin was found in the striatum and cerebellum. Parvalbumin and cholinergic immunoreactive neurons in the striatum have been proven to be particularly vulnerable to perinatal asphyxia [15] (see chapter 6). These data again illustrate that an increase in $\mathrm{NO}$ can play an important role in signaling transduction pathways after injury. In the cerebellum, nNOS is localized in glutamatergic granule cells as well as GABAergic basket cells [28]. It is yet unknown whether cells in the cerebellum are vulnerable to perinatal aphyxia.

In conclusion, our results provide evidence for an increase DAF-2/NO fluorescence and cGMP levels in the striatum in the first 8 days after the perinatal asphyctic insult without marked gliosis. Moreover, these data suggest an increase in NO synthesis during the first 8 days after the insult, indicative for a long-lasting damaging reaction. Furthermore, DAF-2/DA is a sensitive method for identifying the distribution of intracellular NO within cells of brain slices. Since the $\mathrm{DAF}=2 / \mathrm{DA}$ method is easy and rapid to apply, it can become an important tool in the early detection of cellular damage.

\section{Abbreviations}

CGMP, guanosine $3^{\prime}, 5^{\prime}$-cyclic monophosphate; CY3, cyanine-3; DAF-2/DA, 4,5diaminofluorescein diacetate; GFAP, glial fibrillary acidic protein; IBMX, isobutyl-methyl xanthime; $-I R$, immunoreactivity; NADPH-d, nicotinamide adenine dinucleotide phosphate diaphorase; nNOS, neuron nitric oxide synthase; NO, nitric oxide; PDE, phosphodiesterase; PV-3, parvalbumin; ROI, region of interest; SNP, sodium nitroprusside; SPA, severe perinatal asphyxia; TBS-T, Tris-buffered salline containing $0.3 \%$ Triton- $X-100$. 


\section{Acknowledgements}

The generous gift of the antiserum against parvalbumin by Dr. Piers Emson is gratefully acknowledged. The authors are grateful to Marjanne Markerink for her expert technical assistance and helpful suggestions.

\section{References}

[1] Beckman, J.S. and Koppenol, W.H. 1996. Nitric oxide, superoxide, and peroxynitrite: the good, the bad, and ugly. Am J Physiol 271:C1424-37.

[2] Brown, L.A., Key, B.J. and Lovick, T.A. 1999. Blo-imaging of nitric oxide-producing neurones in slices of rat brain using 4,5-diaminofluorescein. I Neurosci Methods 92:101-10.

[3] Buisson, A., Margaill, I., Callebert, J., Plotkine, M. and Boulu, R. G. 1993. Mechanisms involved in the neuroprotective activity of a nitric oxide synthase inhibitor during focal cerebral ischemia. J Neurochem 61:690-6.

[4] Capani, F., Loidl C.F., Aguirre, F., Piehl, L., Facorro, G., Hager, A., Die Paoli, T., Farach, H. and Pecci-Saavedra, J. 2001. Changes in reactive oxygen species (ROS) production in rat brain during global perinatal asphyxia: an ESR study. Brain Res 914:204-7.

[5] Dawson, T.M., Dawson, V.L. and Snyder, S.H. 1994. Molecular mechanisms of nitric oxide actions in the brain, Ann N $Y$ Acad Sci 738:76-85.

[6] de Vente, J., Markerink-van Ittersum, M., van Abeelen, J., Emson, P.C., Axer, H. and Steinbusch, H.W.2000. NO-mediated cGMP synthesis in cholinergic neurons in the rat forebrain: effects of lesioning dopaminergic or serotonergic pathways on nNOS and cGMP synthesis. Eur J Neurosci 12:507-19.

[7] de Vente, J., Steinbusch, H.W. and Schipper, J. 1987. A new approach to immunocytochemistry of $3^{\prime}, 5^{\prime}$-cyclic guanosine monophosphate: preparation, specificity, and initial application of a new antiserum against formaldehyde-fixed $3^{\prime}, 5^{\prime}$-cyclic guanosine monophosphate. Neuroscience 22:361-73.

[8] Dell'Anna, E., Chen, Y., Engidawork, E. Andersson, K., Lubec, G. Luthman, J. and HerreraMarschitz, M. 1997. Delayed neuronal death following perinatal asphyxia in rat. Exp Brain Res 115:105-15.

[9] Garthwaite, J. and Boulton, C.L. 1995. Nitric oxide signaling in the central nervous system. Annu Rev Physiol 57:683-706.

[10] Groenendaal, F., Mishra, O.P., McGowan, J.E. Hoffman, D.J. and Delivoria-Papadopoulos, M. 1996. Cytosolic and membrane-bound cerebral nitric oxide synthase activity during hypoxia in cortical tissue of newborn piglets. Neurosci Lett 206:121-4.

[11] Herbison, A.E., Simonian, S.X., Norris, P.J. and Emson, P.C. 1996. Relationship of neuronal nitric oxide synthase immunoreactivity to $\mathrm{GnRH}$ neurons in the ovariectomized and intact female rat. $\rfloor$ Neuroendocrinol $8: 73-82$.

[12] Igarashi, J., Thatte, H.S., Prabhakar, P., Golan, D.E. and Michel, T. 1999. Calci um-independent activation of endothelial nitric oxide synthase by ceramide. Proc Natl Acad Sci U S A 96:12583-8.

[13] Jourd'heuil, D. 2002. Increased nitric oxide-dependent nitrosylation of 4,5-diaminofluorescein by oxidants: implications for the measurement of intracellular nitric oxide. Free Radic Biol Med 33:676. 
[14] Knowles, R.G., Palacios, M.,Palmer, R.M. and Moncada, S. 1989. Formation of nitric oxide from L-arginine in the central nervous system: a transduction mechanism for stimulation of the soluble guanylate cyclase. Proc Natl Acad Sci U S A 86:5159-62.

[15] Kohihauser, C. Mosgoeller, W. Hoeger, H., Lubec, G. and Lubec, B. 1999. Cholinergic, monoaminergic and glutamatergic changes following perinatal asphyxia in the rat. Cell Mol Life Sci $55: 1491-501$.

[16] Kojima, H., Nakatsubo , N., Kikuchi, K., Kawahara, S., Kirino, Y., Nagoshi, H., Hirata, Y. and Nagano, T. 1998. Detection and imaging of nitric oxide with novel fluorescent indicators: diaminofluoresceins, Anal Chem 70:2446-53.

[17] Kojima, H., Sakural, K., Kikuchi, K., Kawahara, S., Kirino, Y., Nagoshi, H., Hirata, Y. and Nagano, T. 1998. Development of a fluorescent indicator for nitric oxide based on the fluorescein chromophore. Chem Pharm Bull (Tokyo) 46:373-5.

[18] Kowall, N.W., Ferrante, R.J., Beal, M.F., Richardson, E.P., Jr, Sofroniew, M.V., Cuello A.C. and Martin, J.B. 1987. Neuropeptide $Y$, somatostatin, and reduced nicotinamide adenine dinucleotide phosphate diaphorase in the human striatum: a combined immunocytochemical and enzyme histochemical study. Neuroscience 20:817-28.

[19] Loidl, C.F., Capani, F., Lopez-Costa, J.J., Selvin-Testa, A., Lopez, E.M., Goldstein, J. and Pecci-Saavedra, J. 1997. Short-term changes in NADPH-diaphorase reactivity in rat brain following perinatal asphyxia. Neuroprotective effects of cold treatment. Mol Chem Neuropathol 31:301-16.

[20] Loidl, C.F., De Vente, J., van Ittersum, M.M., van Dijk, E.H., Vles, J.S., Steinbusch, H.W. and Blanco, C.E. 1998. Hypothermia during or after severe perinatal asphyxia prevents increase in cyclic GMP-related nitric oxide levels in the newborn rat striatum. Brain Res $791: 303-7$.

[21] Lopez-Figueroa, M.O., Caamano, C., Marin, R., Guerra, B., Alonso, R., Morano, M.I., Akil, H. and Watson, S.J. 2001. Characterization of basal nitric oxide production in living cells. Biochim Biophys Acta 1540:253-64.

[22] Lopez-Figueroa, M.O., Caamano, C., Morano, M.I., Ronn, L.C., Akil, H. and Watson, S.J, 2000. Direct evidence of nitric oxide presence within mitochondria. Biochem Biophys Res Commun 272:129-33.

[23] Lowenstein, C.J. and Snyder, S.H. 1992. Nitric oxide, a novel biologic messenger. Cell 70:705-7.

[24] Lubec, B., Kozlov, A.V., Krapfenbauer, K., Berger, A., Hoeger, H., Herrera-Marschitz, M., Nohl, $H_{.}, K_{0 e c k} T$, and Lubec, G. 1999. Nitric oxide and nitric oxide symthase in the early phase of perinatal asphyxia of the rat. Neuroscience $93: 1017-23$.

[25] Murad, F., Mittal, C.K., Arnold, W.P. Katsuki, S. and Kimura, H. 1978. Guanylate cyclase: activation by azide, nitro compounds, nitric oxide, and hydroxyl radical and inhibition by hemoglobin and myoglobin. Adv Cyclic Nucleotide Res 9:145-58.

[26] Nagata, N., Momose, K. and Ishida, Y. 1999. Inhibitory effects of catecholamines and antioxidants on the fluorescence reaction of 4,5-diaminofluorescein, DAF-2, a novel indicator of nitric oxide. J Biochem (Tokyo) 125:658-61.

[27] Nowicki, J.P., Duval, D., Poignet, H. and Scatton, B. 1991. Nitric oxide mediates neuronal death after focal cerebral ischemia in the mouse. Eur J Pharmacol 204:339-40.

[28] Rodrigo, d., Springall, D.R., Uttenthal, O., Bentura, M.L. "Abadia-Molina, F. Riveros-Moreno, $V_{*}$ Martinez-Murillo, $R_{n}$. Polak, J.M. and Moncada, S. 1994. Lacalization of nitric oxide synthase in the adult rat brain. Philos Trans. R Soc Lond B Biol Sci 345:175-221.

Roychowdhury, S., Luthe, A., Keilhoff, G., Wolf, G. and Hom, T.F. 2002. Oxidative stress in glial cultures: detection by DAF-2 fluorescence used as a tool to measure peroxynitrite rather than nitric oxide. Glia 38:103-14. 
[30] Strijbos, P.J. 1998. Nitric oxide in cerebral ischemic neurodegeneration and excitotoxicity. Crit Rev Neurobiol 12:223-43.

[31] Suzuki, N., Kojima, H., Urano, Y., Kikuchi, K., Hirata, Y. and Nagano, T. 2002. Orthogonality of Calcium Concentration and Ability of 4,5- Diaminofluorescein to Detect NO. J Biol Chem $277: 47-9$.

[32] Van de Berg. W.D., Blokland, A., Cuello, A.C., Schmitz, C., Vreuls, W., Steinbusch, H.W. and Blanco, C.E. 2000. Perinatal asphyxia results in changes in presynaptic bouton number in striatum and cerebral cortex-a stereological and behavioral analysis. J Chem Neuroanat $20: 711-82$.

[33] Van de Berg, W.D., Schmitz, C., Steinbusch, H.W. and Blanco, C.E. 2002. Perinatal asphyxia induced neuronal loss by apoptosis in the neonatal rat striatum: a combined TUNEL and stereological study. Exp Neurol 174:29-36.

[34] Vincent, S.R. and Hope, B.T. 1992. Neurons that say NO. Trends Neurosci 15:108-13.

[35] Vincent, S.R. and Kimura, H. 1992. Histochemical mapping of nitric oxide symthase in the rat brain. Neuroscience 46:755-84.

[36] Wink, D.A., Nims, R.W., Darbyshire, J.F., Christodoulou, D., Hanbauer, I., Cox, G.W., Laval, F., Laval, J., Cook, J.A., Krishna, M.C. and et al. 1994. Reaction kinetics for nitrosation of cysteine and glutathione in aerobic nitric oxide solutions al neutral $\mathrm{pH}$. Insights into the fate and physiological effects of intermediates generated in the $\mathrm{NO} / \mathrm{O} 2$ reaction. Chem Res Toxicol $7: 519-25$

[37] Zhang, X., Kim, W.S., Hatcher, N., Potgieter, K., Moroz, L.L., Gillette, R. and Sweedier, J.V. 2002. Interfering with nitric oxide measurements: 4,5-diaminofluorescein reacts with dehydroascorbic acid and ascorbic acid. J Biol Chem. 
$-118$ 


\section{Impact of perinatal asphyxia on the GABAergic and locomotor system}

W.D.J. Van de Berg, M. Kwaijtaal, A.J.A. De Louw, N.P.A. Lissone, C. Schmitz, R.L.M. Faull, A. Blokland, C.E. Blanco, H.W.M. Steinbusch Neuroscience 2003;117:83-96 


\section{Abstract}

Perinatal asphyxia can cause neuronal loss and depletion of neurotransmitters within the striatum. The striatum plays an important role in motor control, sensorimotor integration and learning. In the present study we investigated whether perinatal asphyxia leads to motor deficits related to striatal damage, and in particular to the loss of GABAergic neurons. Perinatal asphyxia was induced in time-pregnant Wistar rats on the day of delivery by placing the uterus horns, containing the pups, in a $37^{\circ} \mathrm{C}$ water bath for $20 \mathrm{~min}$. Three motor performance tasks (open field, grip test and walking pattern) were performed at 3 and 6 weeks of age. Antibodies against calbindin and parvalbumin were used to stain GABAergic striatal projections neurons and interneurons, respectively. The motor tests revealed subtle effects of perinatal asphyxia, i.e. small decrease in motor activity. Analysis of the walking pattern revealed an increase in stride width at 6 weeks of age after perinatal asphyxia. Furthermore, a substantial loss of calbindin-immunoreactive (-22\%) and parvalbuminimmunoreactive $(-43 \%)$ cells was found in the striatum following perinatal asphyxia at two months of age. GABAA receptor autoradiography revealed nochanges in GABA binding activity within the striatum, globus pallidus or substantia nigra. We conclude that perinatal asphyxia resulted in a loss of GABAergic projection neurons and interneurons in the striatum without alteration of GABAA receptor affinity. Despite a considerable loss of striatal neurons, only minor deficits in motor performance were found after perinatal asphyxia. 


\section{Introduction}

Perinatal asphyxia is an important cause of chronic neurological disability including spasticity, epilepsy and mental retardation in later life $[2,33]$. However, the precise relationship between specific neurochemical, structural and behavioural changes remains unclear. The striatum plays an important role in motor control, sensorimotor integration, and learning [9]. It receives excitatory input from the entire cerebral cortex and projects to both the substantia nigra and globus pallidus $[7,61,77]$. The primary neuronal population within the striatum is the medium spiny $\gamma$-aminobutyric acid (GABA) -ergic neurons which project to the globus pallidus and substantia nigra pars reticulata [15]. Most other neurons within the striatum are considered to be aspiny interneurons containing GABA/parvalbumin [19], somatostatin, neuropeptide Y [68], acetycholine [46], NADPH-diaphorase [47] or substance P [42].

Recent animal studies have indicated differences in the vulnerability of different striatal cell types to neonatal hypoxia-ischemia and perinatal asphyxia. NADPH-diaphorase and somatostatin interneurons in the striatum seem to be relatively resistant to hypoxia-ischemia induced cell death in the neonatal rat $[18,43,48]$, whereas striatal cholinergic interneurons seem to be more susceptible [44]. Dopamine, glutamate and aspartate levels in the rat striatum have been shown to be decreased after perinatal asphyxia [51]. Furthermore, repeated asphyxia in fetal sheep severely decreased calbindin-IR and parvalbumin-IR neurons, but not somatostatin-IR neurons in the striatum [55]. The effect of perinatal asphyxia on the total number of GABAergic projection neurons or GABA/parvalbumin-IR interneurons in the developing rat striatum is unknown.

GABA, amongst other neurotransmitters, is considered to be a key player in the regulation of neurogenesis, cell migration and apoptosis during early postnatal life $[6,36,54]$. Degeneration of GABAergic neurons and/or disruption of GABA receptor functions during early brain development can have long-lasting effects. For example, exposure to a GABAA antagonist during early postnatal development leads to a decrease in the total number of parvalbumin-immunoreactive interneurons in the striatum, suggesting that GABA increases the total number of striatal neurons via a GABAA receptor mediated mechanisin [54]. Furthermore, loss of GABA or impairment of GABA function can lead to profound changes in the control of movement, as illustrated in Huntington's disease [16]. Huntington's disease is, among others, characterized by a major loss of both GABA/enkephalin and GABA/ substance $P$ interneurons in the striatum $[1,21,62]$.

Experimental studies investigating behavioural changes and neurologic outcome of perinatal asphyxia report impairments in motor activity, anxiety and long-term memory performance $[32,35,73]$. The behavioural outcome, however, depends strongly on the animal model used and time point of investigation. For example, postnatal anoxia $\left(100 \% \mathrm{~N}_{2}\right.$ for $25 \mathrm{~min}$ at approximately $30 \mathrm{~h}$ after birth) induced transitory hyperactivity in the open field task between postnatal day (P) 25 and 40 and permanent spatial memory deficits $[37,69]$. By contrast, at term induced perinatal asphyxia resulted in hypoactivity in the open field at 5 months of age and deficits in long-term memory performance at 18 months of age $[50,73]$. Furthermore, 
in the same model anxiety-related behaviour was reduced at 3 months of age [35].

We have previously shown that perinatal asphyxia, induced at term, leads to neuronal los: in the striatum [74]. In the present study, we investigated whether perinatal asphyxia leads to striatal dys function and a loss of the total number of $\mathrm{GABAergic}$ medium-sized projection neurons and/or interneurons in the striatum and frontal cortex. Striatal function was studied using sensorimotor tasks, including open field task, grip test and foot print task. GABAergic projection neurons and interneurons were identifed using antibodies against calbindin and parvalbumin, respectively. Furthermore, we investigated changes in GABAA receptor binding activity as as consequence of perinatal asphyxia in the projection areas of striatal GABAergic neurons, such as the globus pallidus and the substantia nigra pars reticulata. A rat model for perinatal asphyxia was used, which reproduces clinical situations where umbilical cord circulation is altered. In this model acidosis, hypercapnia and hypoxia are present in the whole animal $[52,53]$.

\section{Materials and methods}

\section{Animals}

Thirty full-term pregnant Wistar rats were purchased from Charles River-Broekmans (Someren, The Netherlands). They were housed at the Animal Care Department of University of Maastricht under a reversed $12: 12$ hours dark:light with lights on at 06:00 p.m. at $20^{\circ} \mathrm{C}$, with free access to standard laboratory chow and water. The ethical board of the Maastricht University approved animal care and procedures.

\section{Induction of perinatal asphyxia}

Perinatal asphyxia was induced as described previously by Loidl and coworkers [49]. Dams were decapitated immediately after one or two pups were delivered vaginally (control vaginal delivery; $C V D$ ), the uterus horns were isolated through an abdominal incision and placed in a water bath at $37^{\circ} \mathrm{C}$ for $20 \mathrm{~min}$ (severe perinatal asphyxia; SPA). Subsequently, the uterus was placed in an incubator $\left(37^{\circ} \mathrm{C}\right.$ ), the pups were removed from the uterus, cleaned and resuscitated by gently padding them on the back. After 5 min the resuscitation stopped, the surviving pups were left to recover for $60 \mathrm{~min}$ in the incubator and placed with a surrogate mother. A total of 10 pups were assigned randomly to a surrogate mother. The survival rate was $\pm 40 \%$ in the SPA group and $100 \%$ in the CVD group. The weight of the pups was measured weekly.

\section{Behavioural analysïs}

Twenty-seven male rat pups (15 CVD pups, 12 SPA pups) were tested in the following behavioural taks at 21 days (P21) and 42 days $(\mathrm{P} 42)$ after birth. 


\section{Open field}

Animals were tested in the open field task as described previously by Loidl and co-workers $[50]$. Spontaneous locomotor activity was studied by placing the rat in the center of a 50 by $50-\mathrm{cm}$ square. The square had a $30-\mathrm{cm}$ high enclosure. A radio was playing softly, providing background noise. Movements of the rat were recorded with a film-camera as soon as the rat was placed in the center of the square. The camera, directly mounted above the open field, was attached to a computer running the Ethovision ${ }^{\text {rw }}$ program (Noldus Equipment, Wageningen, the Netherlands). The whereabouts of the rat in the live image acquired by the program were converted to $\mathrm{X}$ and $\mathrm{Y}$ coordinates and stored in a data-file. All rats were placed in the open field for $30 \mathrm{~min}$, and the distance moved and mean velocity was measured every $5 \mathrm{~min}$. Both total distance moved and mean velocity within the $30 \mathrm{~min}$, as differences between $5 \mathrm{~min}$ intervals were analysed.

\section{Grip test}

Muscle strength was measured using the grip test. Rats were placed on a horizontal wire 30 $\mathrm{cm}$ above the surface. The rats were introduced to the wire such that both forepaws came in contact with the wire and there was equal chance of grasping the wire. After grasping the wire, the rat was released and the time elapsed before it fell off was measured, with a maximum duration of $60 \mathrm{~s}$. A five-point scale was used to score whether a rat reached with one forepaw (1) or two forepaws (2). Furthermore, the rat was scored three when the rat used its hind paws but did not climb onto the wire, was scored four when it used its hind paws to climb onto the wire and was scored five when it used its tail to climb onto the wire.

\section{Foot print test}

For evaluation of walking pattern, the footprint task was performed as described previously $[12,45,71]$. The hind paws of the rats were dipped in black body paint and placed in a walking corridor (length $150-\mathrm{cm}$, width $12-\mathrm{cm}$ ) covered with white paper. A light was placed at the starting-point of the corridor, at the end of the corridor was a dark cave.

Five footprints of each trial were analysed using a computerized footprint analysis programme (Footprints, Version 1.22). The following parameters were measured: area touched per foot, foot length, spreading toes $1-5$, spreading toes $2-4$, stride width and stride length (Figure 1 ).

\section{Morphological analysis}

\section{Tissue preparation}

At P56, 11 male rats (six CVD pups and five SPA pups) were chosen at random and perfused through the heart for immunohistochemistry. Rats were anaesthetized with sodium pentobarbital (60mg/kg; i.p.; NembutalB) and perfused with Tyrode $(0.1 \mathrm{M})$ followed by the fixative containing $4 \%$ paraformaldehyde, $15 \%$ picric acid and $0.05 \%$ glutaraldehyde in $0.1 \mathrm{M}$ phosphate buffer ( $\mathrm{pH} 7.6$ ). Brains were removed from the skulls and post fixed overnight at $4^{\circ} \mathrm{C}$ in the fixative omitting the glutaraldehyde. Brain tissue was cryo-protected by immersion 
in $30 \%$ sucrose / $0.1 \mathrm{M}$ tris buffered saline (TBS, pH 7.4 ) for 48 hours at $4^{\circ} \mathrm{C}$. Another 12 male rat pups (six CVD pups and six SPA pups) were decapitated and processed for receptor autoradiography. The brains of all animals were quickly frozen and stored at$80^{\circ} \mathrm{C}$ until further processing. Tissue was sectioned in the coronal plane of section on a cryostat at $30 \mu \mathrm{m}$ for immunocytochemistry and at $16 \mu \mathrm{m}$ for receptor autoradiography.

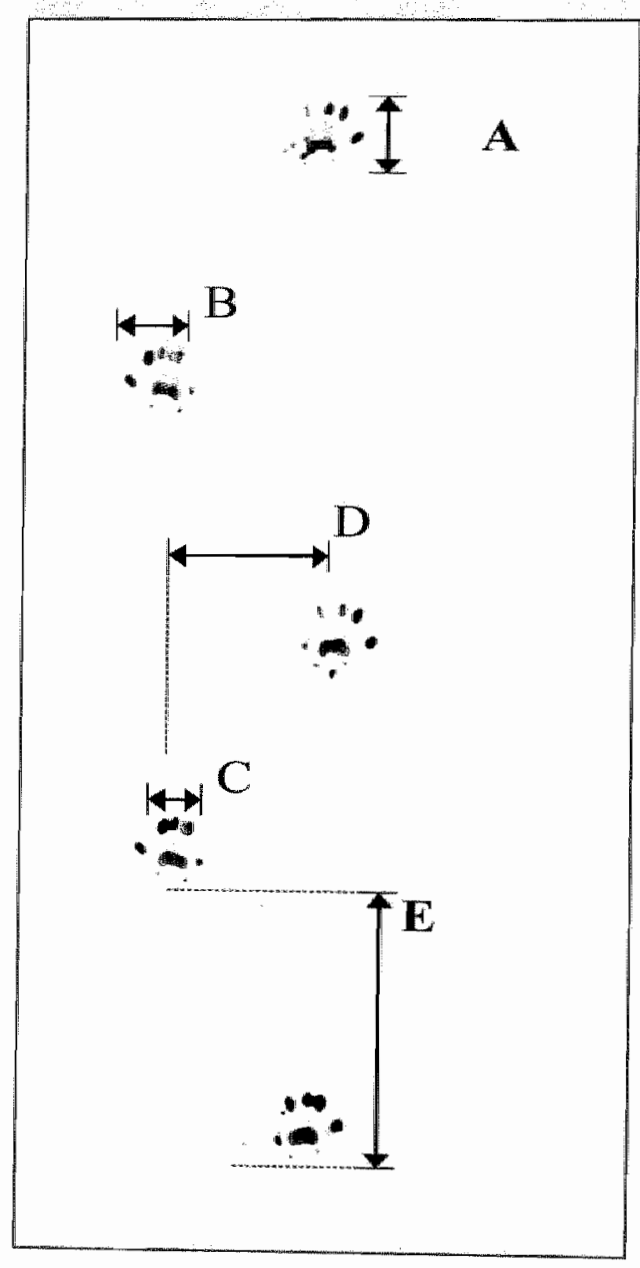

Figure 1. Image of footprint of a control rat at 6 weeks of age. Variables measured were: (A) foot length; (B) spreading toe $1-5 ;(C)$ spreading toe $2-4$; (D) stride width, i.e. distance be-
tween two subsequent feet on the x-axis; (E) stride length, i.e. distance between two subsequent feet on the $y$-axis. In addition, the area touched (i.e. total surface of foot in back ink) was measured (not indicated in figure). All variables were measured in centimeters. 


\section{Immunohistochemistry}

Immunohistochemistry for calbindin and parvalbumin was performed using free-floating sections $(30 \mu \mathrm{m})$. All sections were washed and rinsed with TBS and TBS with $0.3 \%$ Triton X100 (TBS-T; pH 7.4). The calbindin-D46 and parvalbumin (PV3) antibodies (P. Emson, Cambridge, UK) were both used at a dilution of $1: 10,000$ in TBS-T [bovine serum albumine (BSA); Sigma]. Sections were incubated overnight at $4^{\circ} \mathrm{C}$ with the primary antibody. After washing with TBS and TBS-T, the sections were immersed in biotinylated donkey anti-rabbit IgG (1:800; Jackson Immunoresearch Laboratories, Inc., PA, USA) for $1.5 \mathrm{~h}$ at room temperature, followed by $\mathrm{ABC}$-kit (1:800; Vectastain, Burlingame, $\mathrm{CA}$ ). The sections were pre-incubated for $10 \mathrm{~min}$ in $0.3 \% 3.3^{\prime}$-diaminobenzidine tetrahydrochloride (DAB, Sigma) and stained for $5 \mathrm{~min}$ in $0.3 \% \mathrm{DAB}$ containing $0.03 \% \mathrm{H}_{2} \mathrm{O} 2$. The sections were washed and mounted on gelatim-coated slides. After air-drying, the sections were dehydrated in ascending concentrations of alcohol, cleared with microclear and coverslipped with Permount.

\section{Brain areas used for stereological analysis of calbindin and parvalbumin}

The frontal cortex and striatum were defined according to Paxinos and Watson [60]. The area between bregma $2.6 \mathrm{~mm}$ anterior and $-0.8 \mathrm{~mm}$ posterior was identified as the frontal cortex. Medially a line was drawn from the dorsal tip of the left-brain side to the top of the corpus callosum. Laterally a line was drawn from the ventral tip of the lateral ventricle to the cortex in an angle of $45^{\circ}$. Anterior and posterior boundaries for the striatum were set at bregma 1.6 $\mathrm{mm}$ and $-0.8 \mathrm{~mm}$. At bregma 1.6 the corpus callosum first crosses the midline, at bregma$0.8 \mathrm{~mm}$ the fornix joins the dienencephalon. Dorsal and lateral boundaries were defined by the corpus callosum; a line drawn from the ventral tip of the lateral ventricle to the rhinal fissure was used as a ventral boundary [73]. The globus pallidus was excluded from the striatum.

\section{Stereology}

For estimates of the total number of IR cells, every $8^{\text {th }}$ section of the brain was analysed using the optical disector. The thickness of every section was determined using a microcator, attached to a computer. To test whether the immunohistochemical staining penetrated the whole section thickness, we analysed 8 sections of the striatal area ad random chosen of three CVD and three SPA pups for both antibodies (calbindin and PV3). We studied five planes ( $3 \mu \mathrm{m}$ thick) throughout the Z-direction (per plane $\sim 90$ optical disectors). There was almost no staining in the middle plane of the section, so we concluded that the immunohistochemical staining did not penetrate the whole section (see figure 2 ). Therefore two focal planes ( $4 \mu \mathrm{m}$ thick) were used in counting the IR cells per disector, the first layer was 1 to $5 \mu \mathrm{m}$ below the top of the section and the second layer was the last $4 \mu \mathrm{m}, 1 \mu \mathrm{m}$ above the bottom of the section. The C.A.S.T.-Grid system (Olympus, Denmark) was used for quantification. The IR cells, which came into focus within approximately 450 systematically randomly spaced disectors, were counted at a final magnification of $x 3,600$ (distance between disectors in mutually orthogonal directions $x$ and $y$ on the sections: $250 \mu \mathrm{m}$ ). The optical disectors had a base area of 1231 
$\mu \mathrm{m}^{2}$. Estimated total numbers of IR cells were calculated from the number of counted neurons and the sampling probalitity $[27,64]$. Sampling was optimised for prevention of type II error probability due to stereological sampling $[30,65,66]$. The precision of the estimated total numbers of neurons was predicted as recently described in detail $[20,64,66]$.

Volume estimates of the striatum and frontal cortex were obtained using the point-counting method and the Cavalieri's principle [28]. The area of the upper surface of each section was measured using the C.A.S.T.-Grid system. Volume estimates were calculated by multiplying the average surface area with the section thickness (post-processing thickness), the number of investigated sections, and a factor of eight since every eighth section was analysed.

\section{Autoradiographic localization of $\mathrm{GABA}_{\mathrm{A}}$ receptor}

Slide-mounted sections $(16 \mu \mathrm{m})$ were processed for the autoradiographic localisation of GABAA/benzodiazepine receptors as previously described by Waldvogel and co-workers [76]. Sections were labelled at $4^{\circ} \mathrm{C}$ for $90 \mathrm{~min}$ by incubation with $1 \mathrm{nM}\left[{ }^{3} \mathrm{H}\right]$ flunitrazepam (FNZ, $88.0 \mathrm{Ci} / \mathrm{mmol}^{\text {; }}$ Amersham, Little Chalfont, England), $1 \mathrm{nM}\left[{ }^{3} \mathrm{H}\right]$ flunitrazepam in the presence of $200 \mathrm{~nm} \mathrm{CL} 218.872$ (high affinity for type I and low affinity for type II benzodiazepine receptors, FNZ2) or with $1 \mathrm{nM}$ of the benzodiazepine receptor antagonist $\left[{ }^{3} \mathrm{H}\right] \mathrm{Ro} 15-1788$ (Ro 15, 70.8 Ci/mmol, New England Nuclear, Boston, USA). The sections were washed twice in $50 \mathrm{mM}$. Tris- $\mathrm{HCl}$ for $1 \mathrm{~min}$ and dipped in distilled water. Sections were air-dried under a fan

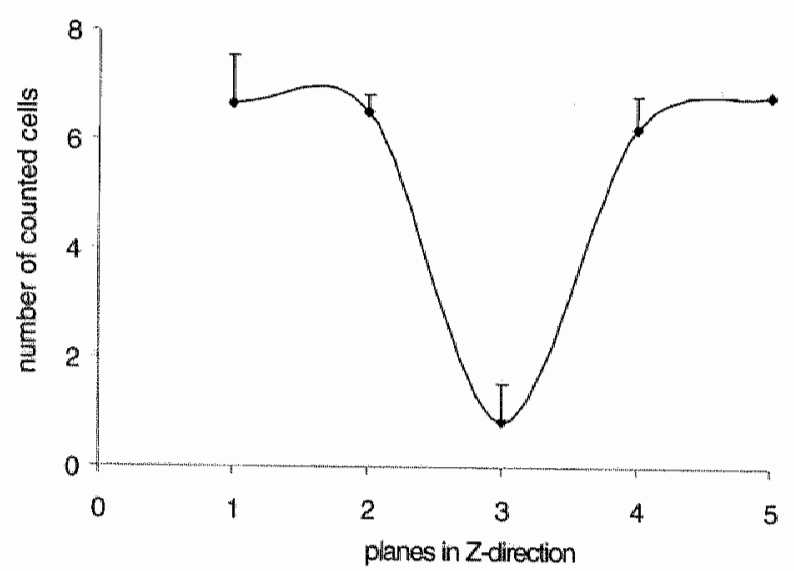

Figure 2. Penetration of the antibodies. The total number of calbindin-IR or parvalbumin-IR cells were counted in 8 sections of the striatal area ad random chosen of three CVD and three SPA rats. Five planes ( $3 \mu \mathrm{m}$ thick) throughout the $Z$-direction (per plane $\sim 90$ disectors: base area of the optical disectors $1231 \mu \mathrm{m}^{2}$ ) were analysed. The mean section thickness was $17 \pm 0.4 \mu \mathrm{m}$. The first and last $1 \mu \mathrm{m}$ of the section was not used for analysis. Since the antibodies were not able to penetrate the whole section (incubated free-floating), there was almost no staining in the middle plane of the section. Results are presented as mean
$\pm S E M$. 
at $4^{\circ} \mathrm{C}$ overnight. A tritium sensitive Hyperfilm (Amersham) was exposed to the slides and placed in X-ray cassettes for 6 weeks. After 6 weeks the film was developed using D 19 (Kodak, Auckland, New Zealland).

\section{Quantitative analysis of autoradiogram}

Densitometric analysis of globus pallidus, striatum and substantia nigra (SN) were made using the MD30 Image Analysis System (Leading Edge Pty, Australia). A distinction between substantia nigra pars compacta (SNc) and substantia nigra pars reticulata (SNr) could not be made so the whole $\mathrm{SN}$ was quantified as one structure. Per animal an average of 25 sections of the striatum, 10 sections of the globus pallidus and 8 sections of the substantia nigra were investigated. The density measurements were converted to 'nCi tritium bound/mg protein' using a standard curve derived from the density reading of the autoradiographic calibration slides; in which $\log \mathrm{nCi} / \mathrm{mg}$ was plotted against $\log$ density $\left(\mathrm{y}=79,151 \mathrm{x}^{1.8084}\right)$. These values were further converted to femtomoles of triated-ligand bound/mg tissue using the known specific activity of the labelled ligand [72].

\section{Statistical analysis}

Results are presented as mean \pm standard error of the means ( \pm SEM). Data of the open field task were analysed by three-factorial (group $x$ interval $x$ age) analysis of variance (ANOVA) with repeated measures over intervals and age. Footprints were analysed using a two-factorial (group $x$ age) ANOVA with age as repeated measures. The grip test was analyzed using the Chi-Square test and a Fishers' exact test, for the measures fall-off latency and strategy, respectively. Stereological and autoradiographical data were analysed using a two-tailed ttest. The level of significance was $\mathrm{P}<0.05$ for all analyses.

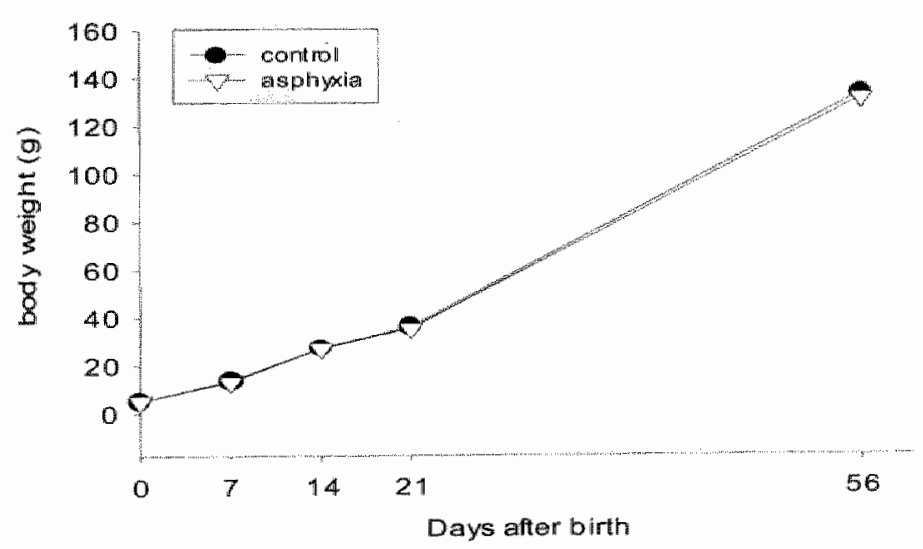

Figure 3. Weights of the rats during the experiment. Closed circles: control rats; Open circles: asphyctic rats. Date are expressed as mean \pm SEM. 


\section{Results}

\section{Body weight and qualitative observations after perinatal asphyxia}

Perinatal asphyxia did not affect the body weight of the rat pups during birth or in the first 2 months after birth (Figure 3 ). Directly after the asphyxia pups were gasping irregularly and had a purple appearance. Pups that were not able to gasp within the first 5 min after the asphyxia were excluded from the study. Asphyctic rat pups were not active in the first $30 \mathrm{~min}$ after birth. Control rat pups had a pink appearance and started moving 5 min after birth. After 60 min in the incubator, there was no visible difference between asphyxia and control rat pups in terms of breathing, colour and movement.

\section{Analysis of behaviour}

\section{Open field}

The locomotor activity of all rats was tested at three and 6 weeks of age. The results of the open field task for the mean distance moved $( \pm S E M)$ and mean velocity $( \pm S E M)$ are given in Figure 4. To remove inhomogeneity of the variances among observations, the data were logarithmically transformed $\left(\log ^{10}\right)$ for analysis. Both groups showed a decline in motor activity $[F(5,125)=9.88$ and $F(5,125)=25.08, P<0.01]$ and mean velocity $[F(5,125)=5.14$ and $\mathrm{F}(5,125)=7.91, \mathrm{P}<0.05]$ during the $30 \mathrm{~min}$ in the open field task at three and 6 weeks of age (Figure 4A and B). A. P21, the mean total distance moved by the SPA rats is only one thind of the distance moved by the CVD rats, However, high interindividual variability among the CVD rats prevented statistical significance. At P42, there was no difference between the mean total distance moved and mean velocity between asphyxia and control group (Figure 4C and 4D). Furthermote, there were no differences in the performance of the rats between intervals. The variance of the data was significantly larger in the control group than in the asphyxia group. There was a tendency for an age $x$ group interaction effect suggesting that the decrease in motor activity was more pronounced in CVD rats than in SPA rats $[\mathrm{F}(1,125)=3.46, \mathrm{P}=0.07]$. A similar effect was found for mean velocity [age $x$ group $F(\mathbb{1}, 125)=2.86, P<0.05$ ].

\section{Grip rest}

Performance of the grip test was tested at 6 weeks of age and depended on the number of repetitions (Table 1). There was no difference in the overall number of scores or fall-off latency between CVD and SPA rats in the three trials. All rats increased the fall-off latency during acquisition of the task.

\section{Foor print analysis}

The effect of perinatal asphyxia on the walking pattern was tested using the footprint task at three and 6 weeks of age (Figure 5). Footprint analysis revealed significant age related change in walking pattern over time for both the CVD and SPA rats on the variables area, foot 
length, spreading toe 1-5 and 2-4, stride width and stride length (Figure 5A-F) [general mean time: $\mathrm{F}>4.5, \mathrm{P}<0.05$ for all variables]. Analysis of stride width revealed a significant increase in width due to the perinatal asphyxia (Figure 5E) [time $x$ group: $F(1,25)=53.5, P<0.01$ ] There was no effect of the perinatal asphyxia on the parameters area, foot length, spreading 1 . 5 and 2-4 or stride length.

A.

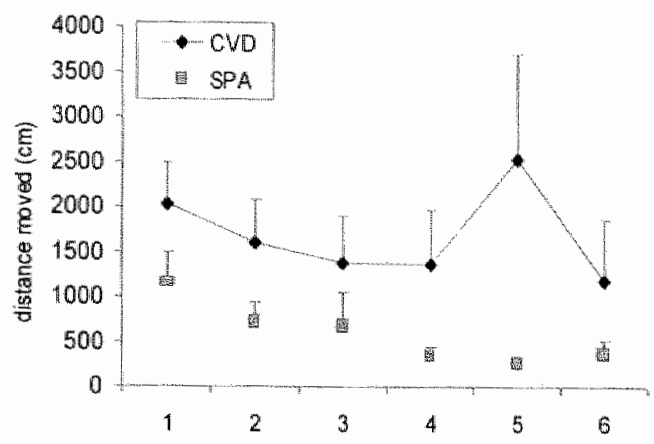

C.

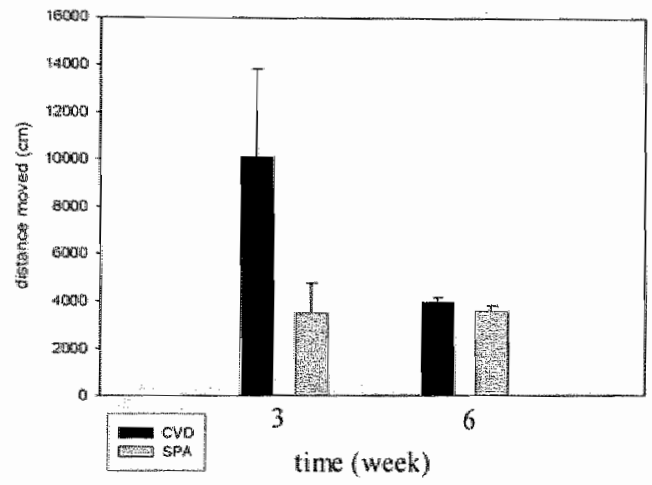

B.

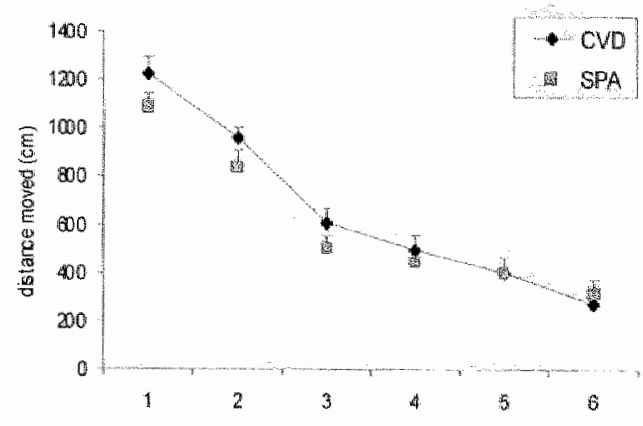

D.

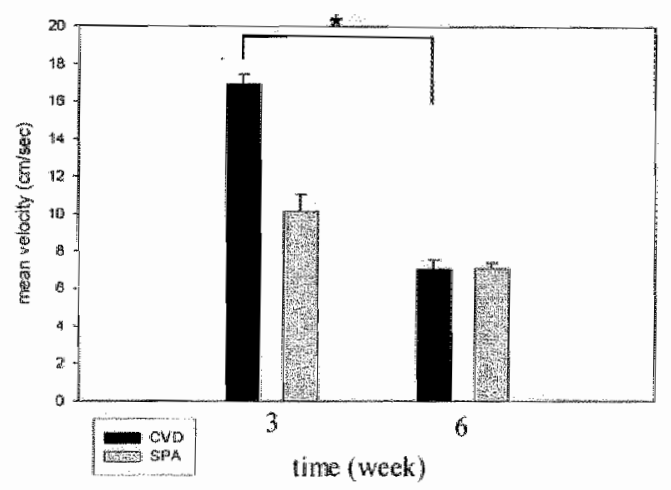

Figure 4. Performance of the three and 6 weeks old rat pups in the open field task. Total distance moved per 5 min interval for CVD (black circle) and SPA (grey square) pups at 3 weeks of age (A) and 6 weeks of age (B). There were no significant differences between the CVD and SPA group. At P21, there was a large inter-individual difference among the CVD rats $(n=15)$. Both groups showed a decline in motor activity during the $30 \mathrm{~min}$ in the open field at 3 and 6 weeks of age. Total distance moved after $30 \mathrm{~min}$ (C) and mean velocity during the $30 \mathrm{~min}$ (D) in the open field task was not different between CVD and SPA rats. However, the decline in mean velocity of the CVD rats was significant larger than the decline in mean velocity of the SPA rats, comparing 3 to 6 weeks of age. Black bar: CVD; grey bar: SPA. Date are expressed as mean \pm SEM. " indicates $P<0.05$. 
A.

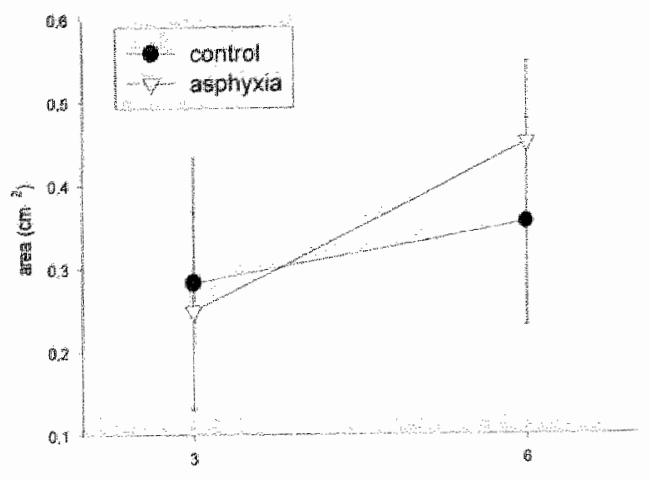

C.

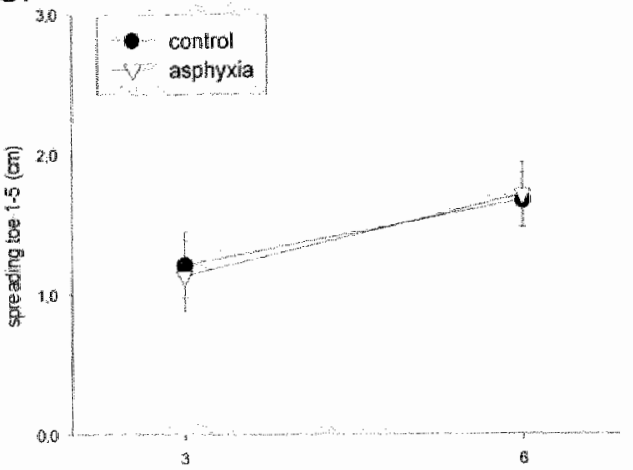

E.

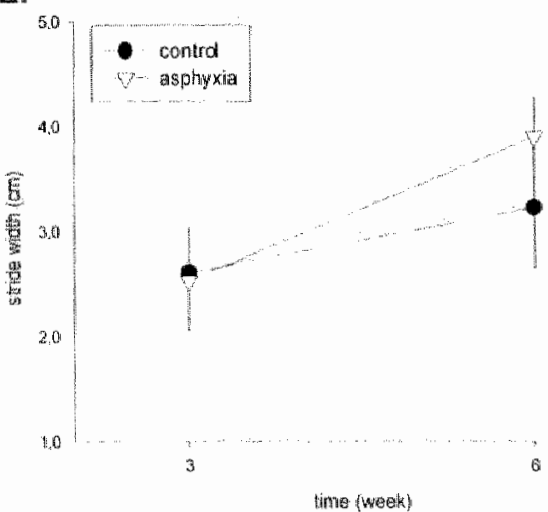

B.

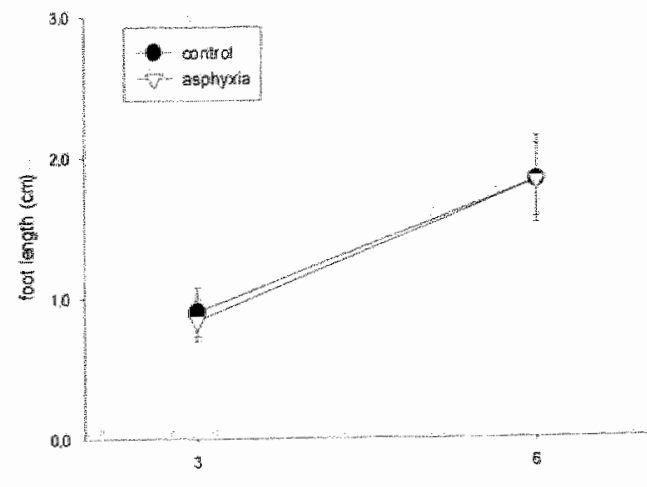

inmo (weetid)
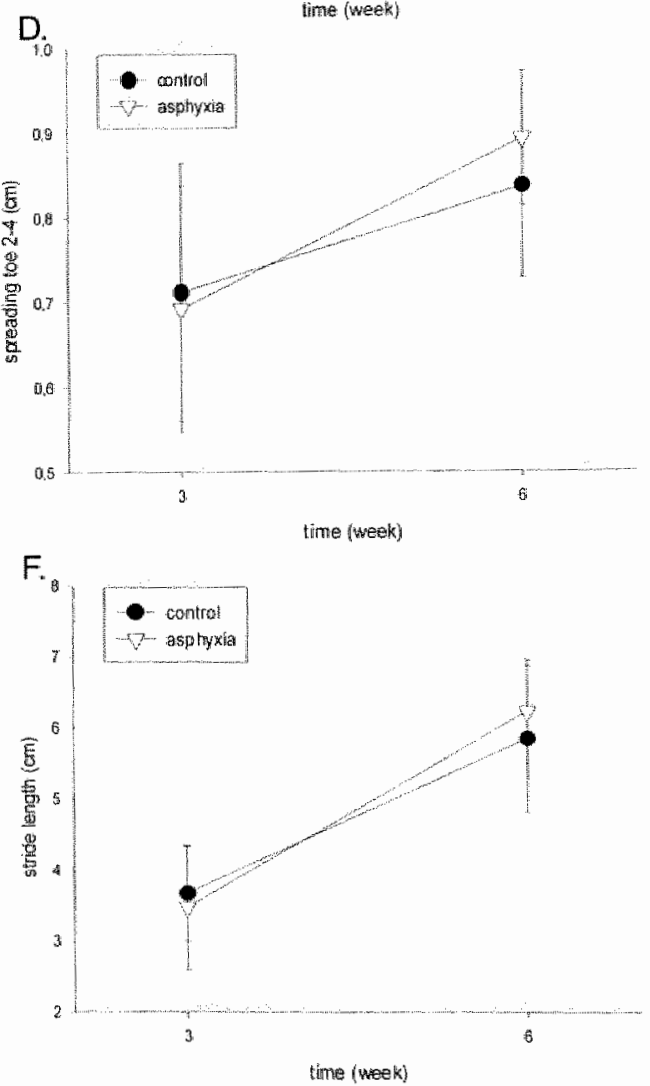

Figure 5. Walking pattern as measured with the footprint analysis. Rats were tested in the footprint analysis at three and 6 weeks of age. Closed circle: control rats (CVD); open circle: asphyctic rats (SPA). (A) Area touched; (B) foot length; (C) spreading toe 1-5 (cm); (D) spreading toes 2-4; (E) stride width; $(F)$ stride length. Data are expressed as mean \pm SEM per group. An age related change was seen in all parameters in both groups $\left(^{*}\right)$. A significant increase in stride width was seen after perinatal asphyxia at 6 weeks of age $(\dagger)$.

* indicates $P<0.05$; † indicates $P<0.01$. 
Table 1. Score of control (CVD; $n=11$ ) and asphyctic (SPA; $n=14$ ) rats in grip task at 6 weeks of age. Data are given as percentages of the total number of rats. Rats were tested 3 times (Trial 1, 2 and 3). Score 1: rat reached with one forepaw. Score 2: rat reached with two forepaws. Score 3: rat used its hind paws. Score 4: rat used its hind paws to climb onto the wire. Score 5: rat used its tail to climb onto the wire. No significant differences were found between groups.

\begin{tabular}{|ccccccc|}
\hline \multirow{2}{*}{ Score } & \multicolumn{2}{c}{ Trial 1 $(\%)$} & \multicolumn{2}{c|}{ Trial $2(\%)$} & \multicolumn{2}{c|}{ Trial 3(\%) } \\
& CVD & SPA & CVD & SPA & CVD & SPA \\
1 & 27.3 & 14.3 & 27.3 & 21.4 & 18.2 & 7.1 \\
2 & 27.3 & 28.6 & 36.3 & 28.6 & 27.3 & 14.3 \\
3 & 27.3 & 35.7 & 27.3 & 28.6 & 27.3 & 21.4 \\
4 & 18.1 & 21.4 & 9.1 & 21.4 & 9.1 & 14.3 \\
5 & 0 & 0 & 0 & 0 & 18.1 & 42.9 \\
\hline
\end{tabular}

\section{Stereology}

The long-term consequence of perinatal asphyxia on the chemospecific cell populations of the striatum and frontal cortex was determined using immunohistochemistry for calbindin and parvalbumin (Figure 6). Estimates of the mean total number of neurons and volumes are presented in Table 2 . In the striatum, a decrease in the mean total numbers of calbindinIR (-22\%) and parvalbumin(PV3)-IR cells $(-43 \%)$ was found in the asphyxia group compared to the control group $(\mathbb{P}<0.05$ for both). In the frontal cortex, a decrease in the mean total number of calbindin-IR cells $(-19.4 \% ; \mathrm{P}<0.05)$ was found in the asphyxia group compared to the control group whereas no change was found in the mean total number of PV3-IR cells (I $1 \%$; n.s.) between the groups. The predicted precision of the estimates was between $0.05-$ 0.11 . Although very large numbers of optical disectors were analysed $(>1200$ per area), only small numbers of immunoreactive cells were found in both the striatum and frontal cortex. Thus, the estimates obtained were based on smaller numbers of counted immunoreactive neurons in the striatum, than recently recommended $[66,67]$. However, all asphyctic rats had smaller total numbers of calbindin-IR cells than the control rats, and the decrease in mean total numbers of calbindin-IR neurons was larger than the predicted precision of the estimates. This demonstrates the appropriateness of the sampling scheme (see [75] for further details). There were no statistically significant differences between the groups in the volume estimates of the investigated brain areas (Table 2). Post-processing thickness of the section was 17.4 $\pm 0.2 \mu \mathrm{m}$. So, the reduction of immunoreactive neurons in the investigated brain regions is not due to differences in volumes, but a reduction in neuronal density. 
Table 2. Estimates of the mean total number of calbindin and parvalbumin (PV3) immunoreactive (IR) neurons in striatum and frontal cortex of control and asphyctic rats. Asphyxia decreased the total number of calbindin-IR neurons in frontal cortex $(-19 \%)$ and striatum $(-22 \%)$. Asphyxia also decreased the total number of PV3-IR in striatum $(-43 \%)$. The volumes of frontal cortex and striatum were unchanged after the asphyxia.

\begin{tabular}{|c|c|c|c|c|c|c|}
\hline \multirow{2}{*}{ Area } & & \multicolumn{2}{|c|}{ Control } & \multicolumn{2}{|c|}{ Asphyxia } & \multirow{2}{*}{$\begin{array}{l}\text { \% c ell } \\
10 \mathrm{ss}\end{array}$} \\
\hline & & mean & SEM & mean & SEM & \\
\hline Frontal & \# calbindin & 707,947 & 48,492 & 570,301 & 26,283 & $-19 \% *$ \\
\hline \multirow[t]{2}{*}{ cortex } & \#V 3 & 175,736 & 3,407 & 157,023 & 23,498 & $-11 \%$ \\
\hline & Volume $\left(\mathrm{mm}^{3}\right)$ & 5.568 & 0.119 & 5.298 & 0.126 & \\
\hline \multirow[t]{3}{*}{ Striatum } & \# calbindin & 716,604 & 46,556 & 562,065 & 52,522 & $-22 \%$ * \\
\hline & \#PV3 & 65,836 & 3,021 & 37,815 & 13,043 & $-43 \%$ \\
\hline & Volume $\left(\mathrm{mm}^{3}\right)$ & 5.579 & 0.079 & 5.553 & 0.162 & \\
\hline
\end{tabular}

$* P<0.05$

\section{Receptor autoradiography}

Quantitative differences in the binding activity of the GABAA receptor in the different projection areas of the striatum were studied using receptor autoradiography. The results of FNZ (A), FNZ2 and Ro15 (C) binding are shown in Figure 7 and 8. Regional distribution of FNZ and Rol 5 was higher in the striatum than in the GP, which was lower than the density in the SN. FNZ2 shows that the GABAA-BZ2 receptors are mainly present in the striatum; the density of FNZ2 is lower in GP and SN than in striatum. In the GP and SN, the GABAA receptors are mainly of the BZ1 type. There was no difference in pattern or distribution between control and asphyctic rats in binding activity of FNZ, FNZ2 or Ro15 $(\mathrm{P}>0.05)$.

\section{Discussion}

In the present study, we investigated whether behavioural deficits following perinatal asphyxia $\left[35,50^{\circ}\right]$ are related to loss of $G A B A$ ergic projection neurons or parvalbumin-immunoreactive interneurons or GABAA receptor affinity. Previously, we reported a neuronal loss within the striatum 3 weeks after perinatal asphyxia [74]. Since the striatum and frontal cortex are involved in co-ordination of locomotion, we expected to observe changes in motor behaviour in asphyctic rats at three and 6 weeks of age. We observed minor deficits in spontaneous locomotor behaviour in the open field and foot print task. In the open field task, a decrease in spontaneous activity in the control group was found when comparing the three to the 6 weeks old rats. 
The asphyctic rats showed a comparable and relatively low spontaneous activity in both open field sessions; hence, it could be argued that the effects of asphyxia in our study were undetected because of so called floor effects. Weight of the animals could be excluded as a confounding factor, since no differences in body weight between control and asphyctic rats were observed at birth, at three or 6 weeks of age. Measurement of walking pattern is considered a sensitive and useful tool for monitoring subtle behavioural changes in relation to striatal degeneration [71]. We found an increase in stride width after asphyxia, indicative of a disturbance in balance or stability when walking. However, all other parameters (area, foot length, spreading toe and stride length) were not changed by perinatal asphyxia. This suggests that the perinatal asplyyxia paradigm used here did not lead to major motor deficits but to very subtle changes.

In the present study, perinatal asphyxia led to a decrease in the mean total number of calbindinIR $(-22 \%)$ and parvalbumin-IR $(-43 \%)$ neurons in the striatum. Furthermore, a decrease ($19 \%$ ) was found in the mean total number of calbindin-IR neurons in the frontal cortex.
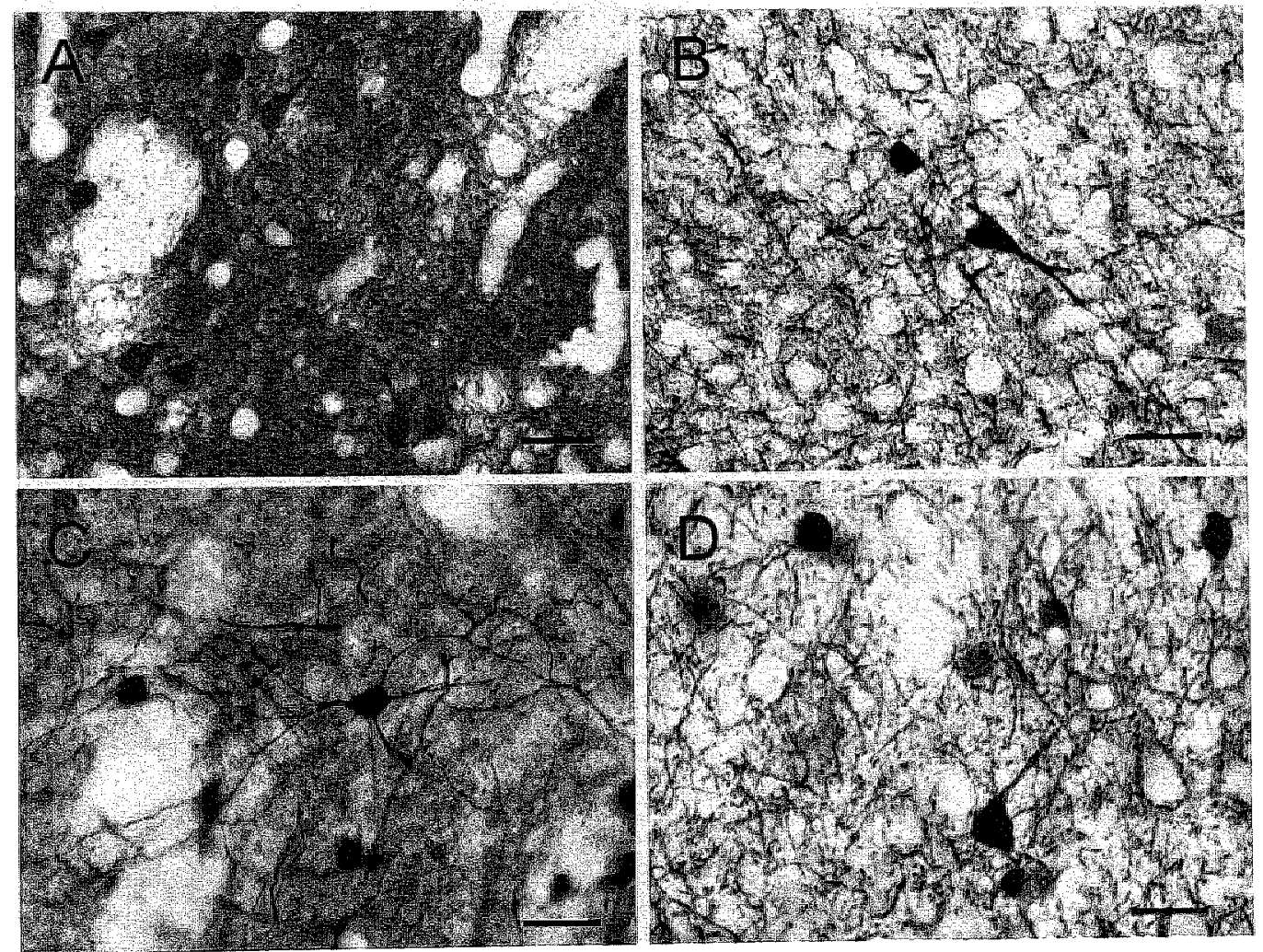

Figure 6. (A and $B$ ) Calbindin immunoreactivity in the striatum ( $A$ ) and 5 th layer of the frontal cortex (B) of 6 weeks old Wistar rats. (C and D) Parvalbumin immunoreactivity in striatum (C) and and 5 th layer of frontal cortex (D). All immunostained nuclei were quantified using stereology. Scale bar is $50 \mu \mathrm{m}$ or $20 \mu \mathrm{m}$. 

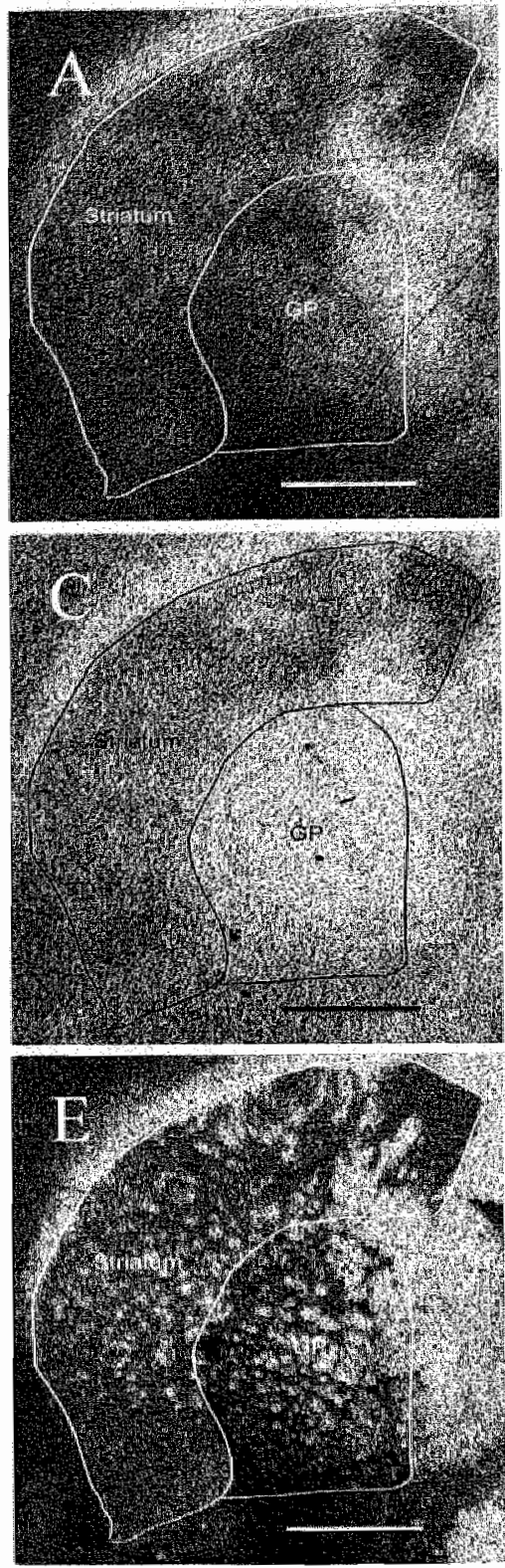
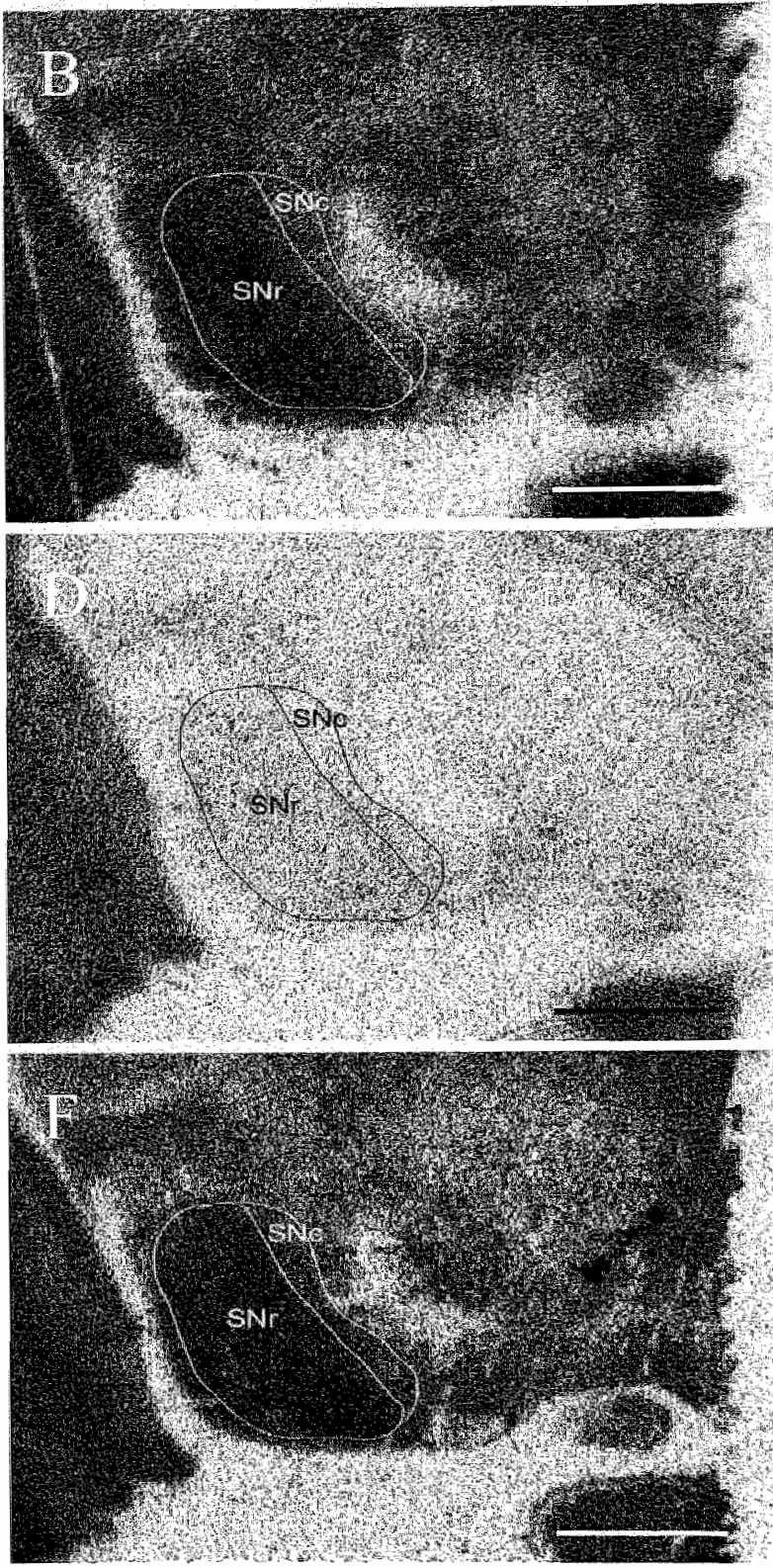

Figure 7. Autoradiograms showing the binding of $\left[{ }^{3} \mathrm{H}\right]$ flunitrazepam ( $\mathrm{FN}$, $\left[{ }^{3} \mathrm{H}\right]$ flunitrazepam in presence of $\mathrm{CL} 218,872$ (FNZ2) and the benzodiazepine receptor antagonist [ $\left.{ }^{3} \mathrm{H}\right] \mathrm{Ro} 15$ 1788 to GABAA receptors in the striatum, globus pallidus (GP) and substantia nigra (SNC and SNr). The density of FN and Ro15 is most pronound in SN. FN2 is mainly present in the striatum. The scalebar represents $1 \mu \mathrm{m}$. 
We did not find a change in density or distribution of the postsynaptic GABAA receptor both within the striatum and in the different projection areas of the striatum. These data suggest that perinatal asphyxia in the rat brain results in a degeneration of both GABAergic projection neurons and interneurons in the striatum. The interneurons are substantially more vulnerable for the asphyctic insult than the GABAergic projection neurons. Since we did not find a change in receptor binding the output centers of the striatum, we hypothesize that the loss of GABAergic projection neurons was compensated for by other neurotransmitter systems on by sprouting of the remaining neurons. Since the GABAA receptor was not affected in the striatum, we propose that the loss of parvalbumin-IR interneurons was compensated for by other interneurons being relatively more resistant to the asphyctic insult. As a consequence, major motor deficits were prevented.

\section{Methodological consideration}

In the present study we provide data concerning the total numbers of calbindin-IR and parvalbumin-IR neurons in the striatum and frontal cortex. The use of stereology allowed precise and valid estimates of the mean total number of calbindin- $\mathbb{R}$ and parvalbumin- $\mathbb{R}$ neurons within the different areas from the control and the asphyxia groups. Since the antibodies were not able to penetrate entirely throughout the section thickness, we analyzed two microscopical virtual planes (each $4 \mu \mathrm{m}$ thick) within the processed sections (thickness after processing was $17.4 \pm 0.2 \mu \mathrm{m}$ ). Luk and Sadikot (2001) studied the total number of parvalbuminIR neurons in the striatum of normal rats between postnatal day 35 and 42 using stereology (optical disector method). They reported 16785 parvalbumin-IR cells in the striatum of Sprague-Dawley rats at 6 weeks of age. We found 65856 parvalbumin-IR cells within the striatum of Wistar rats at 2 months of age. This large difference might be due to the use of different rat strains or time point of investigation. Thus far, there are no studies reporting total numbers of calbindin-IR neurons in the rat striatum. Comparing the mean total number of parvalbumin-IR cells to the mean total number of calbindin-IR cells shows that the mean total number of parvalbumin-IR interneurons within the striatum was approximately $9.2 \%$ of the total number of calbindin-IR projection neurons. Anatomical studies show that the spiny projection neurons represent over $90 \%$ of the total number of cells in the striatum and give rise to almost all outputs of this structure. Our data are in line with this report concerning the ratio between interneurons and projection neurons within the striatum. Furthermore, our data are in line with our previous study, reporting a decrease in mean total number striatal neurons of $16 \%$, three weeks after perinatal asphyxia [74]. The total number of striatal neurons reported in this paper is, however, substantially larger that the total number of projection neurons reported in this study. This is probably due to different proceedings concerning the delineation of the striatum, i.e. in the first study we studied the total number of neurons in the whole striatum and in this study we excluded the globus pallidus and more caudal region of the striatum. 


\section{Neurodegeneration induced by perinatal asphyxia}

Perinatal asphyxia induces the degeneration of several cell types and causes changes in neurotransmitter levels within the basal ganglia $[13,26,43,51]$. Increased extracellular dopamine levels and decreased glutamate levels in the striatum after perinatal asphyxia, induced intrauterine at $\mathrm{PO}$, were reported by microdialysis at 6 months of age [51]. Furthermore, a decrease of GABA levels has been reported in the same study within the substantia nigra. However, $\mathrm{CABA}$ levels were unchanged in the striatum or frontal cortex at three and 6 months of age in the model investigated here [43]. In other models of perinatal asphyxia, loss of $\mathrm{GABAergic}$ neurons was reported in striatum and hippocampus. For instance, loss of glutamate acid decarboxylase (GAD; indicator of GABA levels) was reported in the striatum of sheep after perinatal asphyxia [26]. Loss of striatal neurons, especially loss of calbindin-IR and parvalbumin-IR neurons, was described in the developing brain of the sheep after repeated episodes of cerebral hypoxia-ischemia [55]. Neonatal anoxia (N2 100\% for $25 \mathrm{~min}$ at $30 \mathrm{~h}$ after birth) reduced the number of $G A B A$ ergic neurons in the hippocampus [14]. In conclusion, these data show that GABAergic neurons are particularly vulnerable for perinatal asphyxia. It is assumed that degeneration of calbindin and parvalbumin-IR neurons after the asphyctic insult is mediated by an intracellular calcium accumulation and DNA degradation or via a decrease in metabolic activity of the cell [38]. In the neonatal rat, perinatal asphyxia lleads to an increased release of glutamate into the extracellular space of the striatum directly after the insult, which may result in excitotoxicity [43].

\section{GABAA receptors}

There are three different types of GABA receptors in the brain: bicuculline-sensitive (GABAA), bicuculline-insensitive and $\mathrm{Cl}^{-}$independent $(\mathrm{GABAB})$ and bicuculline- and baclofen-insensitive (GABAC) receptors [34,39]. Activation of the postsynaptic GABAA receptors by synaptically released $G A B A$ inhibits the activation of $G A B A$ ergic spiny neurons and the release of glutamate in the striatum $[57,58]$. GABAB receptors are involved in presynaptic regulation and their stimulation reduces $\mathrm{Ca}^{2+}$ channel conductance [63]. This results in less $\mathrm{Ca}^{2+}$ within the presynaptic cell and results in a decreased release of the neurotransmitter [56]. Behavioural findings suggest a complex interplay between adenosine $\mathrm{A} 2 \mathrm{~A}$, dopamine and $\mathrm{GABA}$ receptormediated mechanisms in the control of motor behaviour [1.7]. Chen and co-workers [10] demonstrated a reduction in the number of dopamine D1 receptors in the nucleus accumbens and substantia nigra, four weeks after perinatal aphyxia. The number of dopamine D2 receptors was unchanged by asphyxia, whereas the D2 agonist affinity was reduced in the striatum. Interestingly, the D2 receptors in the striatum are inhibitory and the D1 receptors are excitatory $[29,70]$. Furthermore, perinatal asphyxia increased mRNA levels of tyrosine hydroxylase (TH), D1 and D2 dopamine receptors in the striatum of 4-week-old rats [25]. Neurotransmitter levels, such as glutamate, GABA, dopamine, were decreased after perinatal asphyxia, although receptors protein levels seem to be less affected. In the present study, we did not detect alterations in GABAA receptor affinity for [ $\mathrm{H} H]$ flunitrazepam or [ $\left.{ }^{3} \mathrm{H}\right] \mathrm{R} 054-864$. These findings suggest that the developing brain does not compensate for the loss in GABA by increasing the affinity or expression of the postsynaptic GABAA receptor. 
FNZ autoradiography

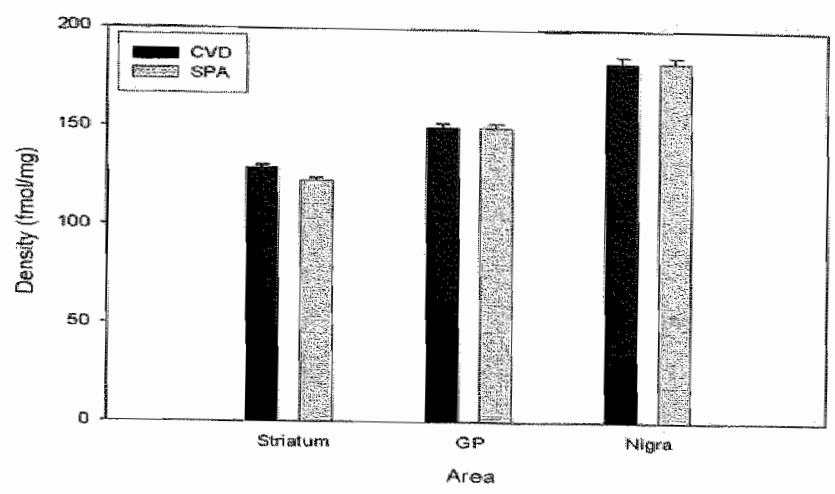

FNZ2 autoradiography

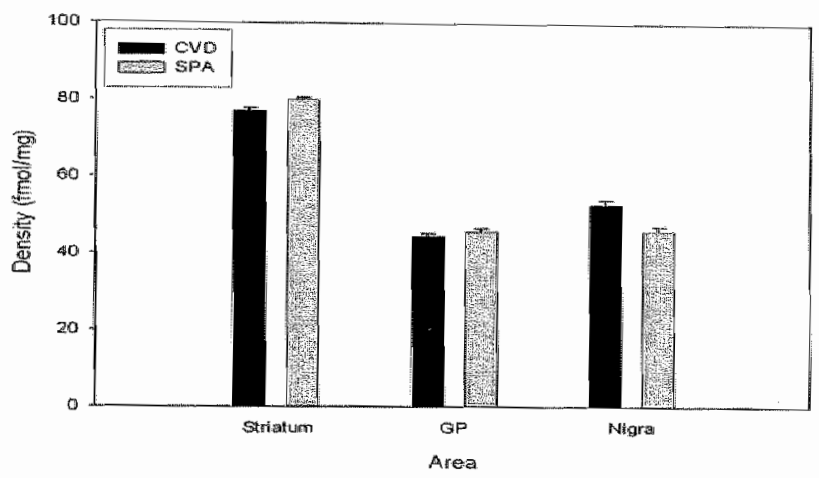

Ro15-1788 authoradiggrapty

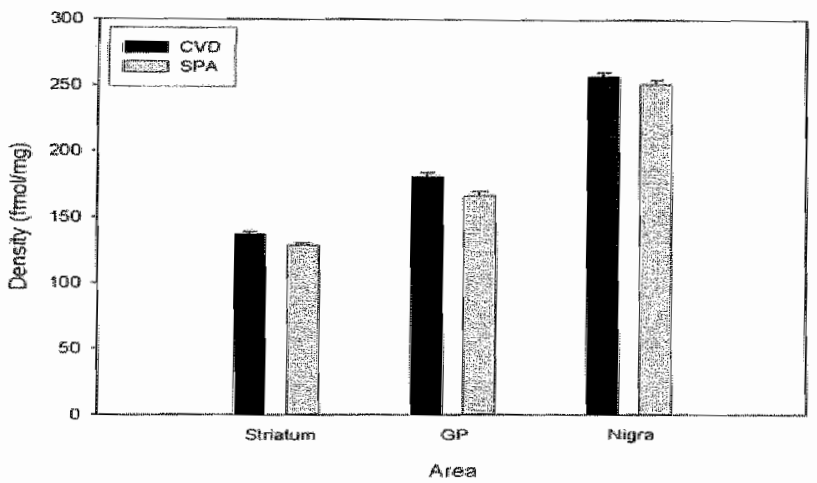

Figure 8. Density of GABAA receptors in the brain of perinatal asphyctic and control rats as a function of the studied brain regions, investigated using receptor autoradiography at 8 weeks of age. Sections were labelled with the GABAA receptor agonists FNZ, FNZ in the presence of CL218.872 (FNZ2) or with the GABAA receptor antagonist Ro15. There was no difference in pattern or distribution between control (black bar) and asphyctic (grey bar) rats in binding activity of the agonists or the antagonist. Date are expressed as mean \pm SEM. 


\section{GABA and development}

GABA is considered to be the main neurotransmitter released from striatal neurons projecting to the different output structures of the basal ganglia. It plays a central role in the processing of information in the striatum [22-24]. During early development, GABA induces depolarization (excitation) in neonatal neurons as a results of high Cl concentration within the cell. During maturation, the $\mathrm{Cl}^{-}$concentration decreases which results in an opposite eflect, i.e. Cl ions are pumped out of the cell and the cell becomes hyperpolarised (inhibition) [40]. This switch occurs in the first week of postnatal life [1 1,31]. Furthermore, in neonatal neurons, the effect of GABA is enhanced by a desensitization of the GABA currents. It is suggested that the GABA receptor changes in structure or assembly of the different subunits during postnatal life. These neurochemical changes lead to modifications in function of the GABAA receptor. It is hypothesized that $\mathrm{GABA}$ exerts trophic actions during early postnatal life by increasing [ $\mathrm{Ca}^{2+}$, which is essential for neuronal grow th and differentiation [59].

Loss of GABAergic cells during early brain development can lead to a distuption of the neuronal network and organization. Ethanol, which interacts with the GABAA receptor, can lead to abnormallities in brain growth when administered during early brain development. GABA is synthesized from glutamic acid by glutamic acid decarboxylase (GAD), which consists of two isoforms with molecular masses of 65 and $67 \mathrm{kDa}$ (GAD65 and GAD67, respectively) [41]. A reduction of GABA in GAD67 /- mice resulted in cleft palate and neonatal death [4]. Loss of GAD65 (GAD65 - / ) , however, did not lead to changes in GABA levels or behavioural changes, except for an increase in susceptibility to seizures [3]. It seems that the brain compensates for the loss of GAD65 and tries to maintain a balance between stimulatory and inhibitory synapses. In our study, we have shown that perinatal asphyxia can lead to a decrease in the number of GABAergic neurons at 2 months of age. Abovementioned studies show that this loss might be compensated during development so that the balance between the excitatory and inhibitory system is not disturbed. Loss of GABAergic neurons during development, however, might affect the total number of neurons or synaptic contacts in later life.

\section{Behavioural changes induced by perinatal asphyxia}

The main function of the basal ganglia is the initiation of movement, or modulation of memoryrelated ol sensorimotor behaviour [22]. Only minor motor deficits were found in the open field and foot print task after perinatal asphyxia, although neuron loss in the striatum was detected using stereology. Our results are in agreement with Hoeger and co-workers [35], who reported no changes in the open field task between asphyctic and control rats at 3 months of age. In the same model, Loidl and co-workers [50] however described a decrease in activity in the open field task at 5 months of age. This difference might be due to the time point of investigation or to the large inter-individual variance at three and 6 weeks of age. At 5 months of age, the inter-individual variance is much smaller and even small changes can be measured. In other studies, using carotid artery ligation at P7 as a model for hypoxia-ischemia, also no changes were also found in the open field task, but a decrease in performance in the grip test and foot print task was reported $[5,8]$. The differences in outcome concerning the grip test 
and foot print task can be related to the different methods used to induce perinatal asphyxia. The control group showed large variations in the performance in the open field task at 3 weeks of age. These results are consistent with previous reports showing large inter-individual differences between rats in behavioural tasks in immature/developing rats.

\section{Concluding remarks}

The present study demonstrates that perinatal asphyxia results in very subtle changes in spontaneous behaviour. GABAA receptor binding was not changed in the different output centers of the striatum after perinatal asphyxia, despite a considerable loss of calbindin- and parvalbumin-IR neurons in the striatum and frontal cortex. Loss of specific neurotransmitters with trophic actions, such as calbindin or parvalbumin, during neonatal life may have important effects on the brain network and the time schedule of events. Studying the mechanisms by which the brain adapts to these changes can give us more inside into interactions between neurotransmitters and developmental disorders of the brain.

\section{Acknowledgements}

We are grateful to Mrs Hellen Steinbusch for technical assistance. Furthermore, we like to thank Judith Dortmans for her assistance with the behavioural tasks. This study was supported by grants from the Health Research Council of New Zealand at the New Zealand Neurological Foundation. 


\section{References}

[1] Albin, R.L., Reiner, A., Anderson, K.D., Dure, L.S.t., Handelin, B., Balfour, R., Whetsell, W.O., Jr., Penney, J.B. and Young, A.B. 1992. Preferential loss of striato-external pallidal projection neurons in presymptomatic Huntington's disease. Ann Neurol. 31: 425-30.

[2] Amiel-Tison, C. and Ellison, P. 1986. Birth asphyxia in the fullterm newborn: early assessment and outcome. Dev Med Child Neural. 28: 671-82.

[3] Asada, H. Kawamura, Y., Maruyama, K., Kume, H., Ding, R., Ji, F., ., Kanbara, N., Kuzume, $\mathrm{H}_{\text {, }}$ Sanbo, M., Yagi, T and Obata, K. 1996. Mice lacking the $65 \mathrm{kDa}$ isoform of glutamic acid decarboxylase (GAD65) maintain normal levels of GAD67 and GABA in their brains but are susceptible to seizures. Biochem Biophys Res Commun. 229: 891-5.

[4] Asada, H., Kawamura, Y., Maruyama, K., Kume, H., Ding, R. G., Kanbara, N., Kuzume, H., Sanbo, $M_{*}$ Yagi, T. and Obata, $K .1997$. Cleft palate and decreased brain gamma-aminobutyric acid in mice lacking the $67-\mathrm{kDa}$ isoform of glutamic acid decarboxylase. Proc Natl Acad Sci U SA. 94: 6496-9.

[5] Balduini, W., De Angelis, V., Mazzoni, E. and Cimino, M. 2000. Long-lasting behavioral alterations following a hypoxic/ischemic brain injury in neonatal rats. Brain Res. 859: 31825.

16] Belhage, B., Hansen, G.H. Elster, L. and Schousboe, A. 1998. Effects of gammaaminobutyric acid (GABA) on synaptogenesis and synaptic function. Perspect Dev Neurobiol. 5: 235-46.

[7] Berendse, H.W. and Groenewegen, H.J. 1991. The connections of the medial part of the subthalamic nucleus in the rat: evidence for a parallel organization. In: The basal gnaglia III (Bernardi, G., Carpenter, M.B., Di Chiara, G., Morelli, M., Stanzione, P., Eds.). New York: Plenum: 89-98.

[8] Bona, E., Johansson, B. B. and Hagberg, H. 1997. Sensorimotor function and neuropathology five to six weeks after hypoxia-ischemia in seven-day-old rats. Pediatr Res. 42: 678-83.

[9] Calabresi, P., De Murtas, M. and Bernardi, G. 1997. The neostriatum beyond the motor function: experimental and clinical evidence. Neuroscience. 78: 39-60.

[10] Chen, Y., Hillefors-Berglund, M., Herrera-Marschitz, M., Bjelke, B., Gross, U., Andersson, $\mathrm{K}$. and won Euler ${ }_{n}$ G. 1997. Perinatal asphyxia induces long-term changes in dopamine D1, $D 2$, and D3 receptor binding in the rat brain. Exp Neurol. 146: 74-80.

[11] Cherubini, E., Gaiarsa, J.L. and Ben-Ari, Y. 1991. GABA: an excitatory transmitter in early postnatal life. Trends Neurosci. 14:515-9.

[12] de Medinaceli, L. Freed, W.J. and Wyatt, R.J. 1982. An index of the functional condition of rat sciatic nerve based on measurements made from walking tracks. Exp Neurol. 77: 63443.

[13] Dell'Anna, E., Chen, Y., Engidawork, E., Andersson, K., Lubec, G., Luthman, J. and HerreraMarschitz, M. 1997. Delayed neuronal death following perinatal asphyxia in rat. Exp Brain Res. 115: $105-15$.

[14] Dell'Anna, E., Geloso, M.C., Magarelli, M. and Molinari, M. 1996. Development of GABA and calcium binding proteins immunoreactivity in the rat hippocampus following neonatal anoxia. Neurosci Lett. 211: 93-6.

[15] DeLong, M.R. 1990. Primate models of movement disorders of basal ganglia origin. Trends Neurosci. 13: 281-5.

[16] Faull, R.L., Waldvogel, H.J., Nicholson, L.F. and Synek, B.J. 1993. The distribution of GABAAbenzodiazepine receptors in the basal ganglia in Huntington's disease and in the quinolinic acid-lesioned rat. Prog Brain Res. 99: 105-23.

[17] Ferre, S., Fredholm, B.B., Morelli, M., Popoli, P. and Fuxe, K. 1997. Adenosine-dopamine 
receptor-receptor interactions as an integrative mechanism in the basal ganglia. Trends Neurosci. 20: 482-7.

[18] Ferriero, D.M., Arcavi, L.J., Sagar, S.M., McIntosh. T.K. and Simon, R.P. 1988. Selective sparing of NADPH-diaphorase neurons in neonatal hypoxia- ischemia. Ann Neurol. 24: 670-6.

[19] Gerfen, C.R., Baimbridge, K.G. and Miller, J.J. 1985. The neostriatal mosaic: compartmental distribution of calcium-binding protein and parvalbumin in the basal ganglia of the rat and monkey. Proc Natl Acad Sci U S A. 82: 8780-4.

[20] Glaser, E.M. and Wilson, P.D. 1998. The coefficient of error of optical fractionator population size estimates: a computer simulation comparing three estimators. J Microsc. 192: 163-71.

[21] Graveland, G.A., Williams, R.S. and DiFiglia, M. 1985. Evidence for degenerative and regenerative changes in neostriatal spiny neurons in Huntïngton's disease. Science. 227: $770-3$.

[22] Graybiel, A.M. 1990. The basal ganglia and the initiation of movement. Riev Neurol. 146: 570-4.

[23] Graybiel, A.M. 1990. Neurotransmitters and neuromodulators in the basal ganglia. Trends Neurosci. 13: 244-54.

[24] Graybiel, A.M., Aosaki, T., Flaherty, A.W. and Kimura, M. 1994. The basal ganglia and adaptive motor control. Science. 265: 1826-31.

[25] Gross, J., Muller, I., Chen, Y., Elizalde, M., Leclere, N., Herrera-Marschitz, M. and Andersson, K. 2000. Perinatal asphyxia induces region-specific long-term changes in mRNA levels of tyrosine hydroxylase and dopamine $D(1)$ and $D(2)$ receptors in rat brain. Brain Res Mol Brain Res. 79: 110-7.

[26] Guan, J., Bennet, T.L., George, S., Waidvogel, H.J., Faull, R.L., Gluckman, P.D., Keunen, H. and Gunn, A.J. 2000. Selective neuroprotective effects with insulin-like growth factor-1 in phenotypic striatall neurons following ischemic brain injury in fetal sheep. Neuroscience. 95 : $831-9$.

[27] Gundersen, H.J. 1986. Stereology of arbitrary particles. A review of unbiased number and size estimators and the presentation of some new ones, in memory of William R. Thompson. J Microsc. 143: 3-45.

[28] Gundersen, H.J. and Jensen, E.B. 1985. Stereological estimation of the volume-weighted mean volume of arbitrary particles observed on random sections. $J$ Microsc. 138: 127-42.

[29] Harsing, L.G., Jr. and Zigmond, M.J. 1997. Influence of dopamine on GABA release in striatum: evidence for D1-D2 interactions and non-synaptic influences. Neuroscience. 77 : 419-29.

[30] Heinsen, H. Rub, U., Bauer, M., Ulmar, G., Bethke, B. Schuler, M., Bocker, F., Eisenmenger, W., Gotz, M., Korr, H. and Schmitz, C. 1999. Nerve cell loss in the thalamic mediodorsal nucleus in Huntington's disease. Acta Neuropathol (Berl). 97: 613-22.

[31] Herlenius, E. and Lagercrantz, H. 2001. Neurotransmitters and neuromodulators during early human development. Early Hum Dev. 65: 21-37.

[32] Hershkowitz, M., Grimm, V.E. and Speiser, Z, 1983. The effects of postnatal anoxia on behaviour and on the muscarinic and beta-adrenergic receptors in the hippocampus of the developing rat. Brain Res. 283: 147-55.

[33] Hill, A. and Volpe, J.J. 1981. Seizures, hypoxic-ischemic brain injury, and intraventricular hemorrhage in the newborn. Ann Neurol. 10: 109-21.

[34] Hill, D.R. and Bowery, N.G. 1981. 3H-baclofen and 3H-GABA bind to bicuculline-insensitive GABA B sites in rat brain. Nature. 290: 149-52.

[35] Hoeger, H., Engelmann, M., Bernert, G., Seidl, R., Bubna-Littitz, H., Mosgoeller, W., Lubec, $B$. and Lubec, G. 2000 . Long term neurological and behavioural effects of graded perinatal asphyxia in the rat. Life Sci. 66: 947-62. 
[36] Ikonomidou, C., Bittigau, P, Koch, $C_{.,}$Genz, K., Hoerster, F, Felderhoff-Mueser, U., Tenkova, T., Dikranian, K. and Olney, J.W. 2001. Neurotransmitters and apoptosis in the developing brain. Biochem Pharmacol. 62: 401-5.

[37] Iuvone, L., Geloso, M.C. and Dell'Anna, E. 1996. Changes in open field behavior, spatial memory. and hippocampal parvalbumin immunoreactivity following enrichment in rats exposed to neonatal anoxia. Exp Neurol. 139:25-33.

[38] Johansen, F.F., Tonder, N., Zimmer, J., Baimbridge, K.G. and Diemer, N.H. 1990. Shortterm changes of parvalbumin and calbindin immunoreactivity in the rat hippocampus following cerebral ischemia. Neurosci Lett. 120: 171-4.

[39] Johnston, G.A.R. 1997. Molecular biology, pharmacology, and physiology of GABAC receptors. In: Enna, S.J., bowery, N.G. (Eds.), The GABA Receptors. Human Press, Totowa, NJ: 297-323.

[40] Kaila, K. and Voipio, J. 1987. Postsynaptic fall in intracellular pH induced by GABA-activated bicarbonate conductance. Nature. 330: 163-5.

[41] Kaufman, D.L., Houser, C.R. and Tobin, A.J. 1991. Two forms of the gamma-aminobutyric acid synthetic enzyme glutamate decarboxylase have distinct intraneuronal distributions and cofactor interactions. J Neurochem. 56: 720-3.

[42] Kim, J.S., Bak, I.J, Hassler, R. and Okada, Y. 1971. Role of -aminobutyric acid (GABA) in the extrapyramidal motor system. 2. Some evidence for the existence of a type of GABArich strio-nigral neurons. Exp Brain Res. 14: 95-104.

[43] Kohihauser, C., Kaehler, S., Mosgoeller, W., Singewald, N. Kouvelas, D., Prast, H., Hoeger, $H$. and Lubec, B. 1999. Histological changes and neurotransmitter levels three months following perinatal asphyxia in the rat. Life Sci. 64:2109-24.

[44] Kohlhauser, C., Mosgoeller, W., Hoeger, H., Lubec, G. and Lubec, B. 1999. Cholinergic, monoaminergic and glutamatergic changes following perinatal asphyxia in the rat. Cell Mol Life Sci. 55: 1491-501.

[45] Kunkel-Bagden, E. and Bregman, B.S. 1990. Spinal cord transplants enhance the recovery of locomotor function after spinall cord injury at birth. Exp Brain Res. 81: 25-34.

[46] Lehmann, J. and Langer, S.Z. 1983. The striatal cholinergic interneuron: synaptic target of dopaminergic terminals? Neuroscience. 10: 1105-20.

[47] Leigh, P.N., Connick, J.H. and Stone, T.W. 1990. Distribution of NA.DPH-diaphorase positive cells in the rat brain. Comp Biochem Physiol C. 97: 259-64.

[48] Loidl, C.F. Capani, F., Lopez-Costa, J.J., Selvin-Testa, A., Lopez, E.M. Goldstein, J. and Pecci-Saavedra, J. 1997. Short-term changes in NADPH-diaphorase reactivity in rat brain following perinatal asphyxia. Neuroprotective effects of cold treatment. Mol Chem Neuropathol. 31:301-16.

[49] Loidl, C.F., De Vente, J., van Ittersum, M.M., van Dijk, E.H., Vles, J.S., Steinbusch, H.W. and Blanco, C.E. 1998. Hypothermia during or after severe perinatal asphyxia prevents increase in cyclic GMP-related nitric oxide levels in the newborn rat striatum. Brain Res. 791: 303-7.

[50] Loidl, C.F."Gavilanes, A.W., Van Dijk, E.H., Vreuls, W., Blokland, A., Vles, J.S., Steinbusch, H.W. and Bilanco, C.E. 2000. Effects of hypothermia and gender on survival and behavior after perinatal asphyxia in rats. Physiol Behav. 68: 263-9.

[51] Loidl, G.F., Herrera-Marschitz, M. Andersson, K., You, Z.B., Goiny, M., O'Connor, W.T., Silveira, R., Rawal, R., Bjelke, B., Chen, $Y$. and el al. 1994. Long-term effects of perinatal asphyxia on basal ganglia neurotransmitter systems studied with microdialysis in rat. Neurosci Lett. 175: 9-12.

[52] Lubec, B., Dell'Anna, E., Fang-Kircher, S., Marx, M., Herrera-Marschitz, M. and Lubec, G. 1997. Decrease of brain protein kinase $\mathrm{C}$, protein kinase $\mathrm{A}$, and cyclin- dependent kinase 
correlating with pH precedes neuronal death in neonatal asphyxia. J Investig Med. 45: 28494.

[53] Lubec, B., Marx, M., Herrera-Marschitz, M., Labudova, O., Hoeger, H., Gille, L., Nohl, H., Mosgoeller, W. and Lubec, G. 1997. Decrease of heart protein kinase C and cyclin-dependent kinase precedes death in perinatal asphyxia of the rat. Faseb J. 11: 482-92.

[54] Luk, K.C. and Sadikot, A.F. 2001. GABA promotes survival but not proliferation of parvalbumin- immunoreactive interneurons in rodent neostriatum: an in vivo study with stereology. Neuroscience. 104: 93-103.

[55] Mallard, E.C., Waldvogel, H.J., Williams, C.E., Faull, R.L. and Gluckman, P.D. 1995. Repeated asphyxia causes loss of striatal projection neurons in the fetal sheep brain. Neuroscience. 65: 827-36.

[56] Matsumoto, R.R. 1989. GABA receptors: are cellular differences reflected in function? Brain Res Brain Res Rev. 14: 203-25.

[57] McKernan, R.M. and Whiting, P.J. 1996. Which GABAA-receptor subtypes really occur in the brain? Trends Neurosci. 19: 139-43.

[58] Mehta, A.K. and Ticku, M.K. 1999. An update on GABAA receptors. Brain Res Brain Res Rev. 29: 196-217.

[59] Miller, R.J. 1988. Calcium signalling in neurons. Trends Neurosci. 11:415-9.

[60] Paxinos, G., Watson, C. 1986. The rat brain in stereotaxic coordinates. Academic press, Sydney.

[61] Plenz, D. and Kitai, S.T. 1998. Up and down states in striatal medium spiny neurons simultaneously recorded with spontaneous activity in fast-spiking interneurons studied in cortex-striatum-substantia nigra organotypic cultures. J Neurosci. 18: 266-83.

[62] Reiner, A., Albin, R.L., Anderson, K.D., D'Amato, C.J., Penney, J.B. and Young, A.B. 1988. Differential loss of striatal projection neurons in Huntington disease. Proc Natl Acad Sci U SA. 85: 5733-7.

[63] Robertson, B. and Taylor, W.R. 1986 . Effects of gamma-aminobutyric acid and (-)-baclofen on calcium and potassium currents in cat dorsal root ganglion neurones in vitro. $\mathrm{Br} J$ Pharmacol. 89: 661-72.

[64] Schmitz, C. 1998. Variation of fractionator estimates and its prediction. Anat Embryol (Berl). 198: 371-97.

[65] Schmitz, C.2000. Towards the use of state-of-the-art stereology in experimental gerontology. Exp Gerontol. 35: 429-31.

[66] Schmitz, C. and Hof, P.R. 2000. Recommendations for straightforward and rigorous methods of counting neurons based on a computer simulation approach. J Chem Neuroanat. 20:93114.

[67] Schmitz, C., Korr, H., Perl, D.P. and Hof, P.R. 2001. Advanced use of 3 D methods for counting neurons. Trends Neurosci. 24: 377-80.

[68] Smith, $Y$. and Parent, A. 1986. Neuropeptide Y-immunoreactive neurons in the striatum of cat and mankey: morphological characteristics, intrinsic organization and co- localization with somatostatin. Brain Res. $372: 241-52$.

[69] Speiser, Z., Korczym, A.D., Teplitzky, I. and Gitter, S. 1983. Hyperactivity in rats following postnatal anoxia. Behav Brain Res. 7: 379-82.

$[70]$ Starr, M. 1987. Opposing roles of dopamine $D 1$ and $D 2$ receptors in nigral gamma$[3 \mathrm{H}]$ aminobutyric acid release? $₫$ Neurochem. 49: 1042-9.

[71] Teunissen, C.E., Steinbusch, H.W., Angevaren, M., Appels, M., de Bruijn, C., Prickaerts, J. and de Vente, J. 2001. Behavioural correlates of striatal glial fibrillary acidic protein in the 3 . nitropropionic acid rat model: disturbed walking pattern and spatial orientation. Neuroscience. 105: $153-67$. 
[72] Unnerstall, J.R., Niehoff, D.L., Kuhar, M.J. and Palacios, J.M. 1982. Quantitative receptor autoradiography using $[3 \mathrm{H}]$ ultrofilm: application to multiple benzodiazepine receptors. $]$ Neurosci Methods, 6: 59-73.

[73] Van de Berg, W.D., Blokland, A., Cuello, A.C., Schmitz, C., Vreuls, W. Steinbusch, H.W. and Blanco, C.E. 2000. Perinatal asphyxia results in changes in presynaptic bouton number in striatum and cerebral cortex-a stereological and behavioral analysis. J Chem Neuroanat. 20:71-82.

[74] Van de Berg, W.D., Schmitz, C., Steinbusch, H.W. and Blanco, C.E. 2002. Perinatal asphyxia induced neuronal loss by apoptosis in the neonatal rat striatum: a combined TUNEL and stereological study. Exp Neurol. 174:29-36.

[75] von Horsien, S., Helfritz, A., Kuhimann, S., Nave, H., Tschernig, T., Pabst, R., Ben-Eliyahu, S., Meyer, D., Schmidt, R.E. and Schmitz, C. 2000. Stereological quantification of carboxyfluorescein-labeled rat lung metastasis: a new method for the assessment of natural killer cell activity and tumor adhesion in vivo and in situ. J Immunol Methods. 239: 25-34.

[76] Waldvogel, H.J., Faull, R.L., Jansen, K.L., Dragunow, M., Richards, J.G., Mohler, H. and Streit, P. 1990. GABA, GABA receptors and benzodiazepine receptors in the human spinal cord: an autoradiographic and immunohistochemical study at the light and electron microscopic levels. Neuroscience. 39: 361-85.

[77] Wilson, C.J. and Kawaguchi, Y. 1996. The origins of two-state spontaneous membrane potential fluctuations of neostriatal spiny neurons. J Neurosci. 16: 2397-410. 


\section{Regional specificity in neuronal and synaptic loss after perinatal asphyxia in the rat brain}

W.D.J. Van de Berg, C. Schmitz, H.P.J Steinbusch, J. Dortmans, J.S.H. Vies, H.W.M Steinbusch, C.E. Blanco

Brain Research, submitted 


\section{Abstract}

Perinatal asphyxia can lead to long-lasting motor and cognitive deficits. We hypothesized that these behavioural deficits were partly the result of a deficient number of synapses within the striatum, hippocampus and/or cerebellum. We therefore quantified the number of neurons and the density of synaptophysin-immunoreactive presynaptic boutons within the striatum, the CA1 and CA3 layers of the hippocampus, and the molecular layer of the cerebellum in rats with a history of global asphyxia at term and controls using stereology and image analysis. Perinatal asphyxia caused a $13 \%$ decrease in the number of hippocampal pyramidal cells at three weeks of age; in contrast the number of Purkinje cells within the cerebellum was not changed. Furthermore, perinatal asphyxia caused a reduction in the number of synapses at two months of age within the striatum, predominantly within its dorso-caudal part. Within the hippocampus, the number of synapses was not affected within the hippocampus, whereas the number of synapses within the cerebellum was slightly reduced. Considering these and previous data from our laboratory, we conclude that perinatal asphyxia induces damages to the brain in a region-specific manner, predominantlly within the striatum. 


\section{Introduction}

Extensive neuronal death and synaptic loss within the developing or aging brain has been associated with functional decline $[29,50,51]$. Moreover, there is a selective vulnerability to which neurons and synapses die and which are resistant to degeneration, especially after brain injury. Brain injury due to perinatal asphyxia can lead to motor and cognitive impairment in adult life $[2,16,53]$. Perinatal asphyxia may result in cell death within the striatum, hippocampus and cerebellum [8,54]. Gamma aminobutyric acid (GABA)-ergic neurons within the striatum are known to be especially vulnerable to perinatal asphyxia mediated cell death [27,55], as well as Purkinje cells within the cerebellum [19,24]. Little is known however about the consequences of cell death after perinatal asphyxia on the number of synapses within these affected areas.

Synaptic plasticity, i.e. the capability of the central nervous system to change the structure, distribution, or number of synapses when required [7], is most prominent during development and is dependent on both external and internal perturbations including changes in hormonal levels and environmental modifications $[30,45]$. The hippocampus seems to be particularly plastic. Changes in the number of synapses within the hippocampus were reported; an increase in synaptic numbers was described after brain-derived neurotrophic factor (BDNF) infusion [52] and watermaze overtraining [39] and a decrease in synaptic numbers after ischemic injury $[17,28]$. Similarly, arborizations of Purkinje cells within the cerebellum seem to be particularly plastic, especially in response to motor activity $[9,37]$.

To establish whether there are region-specific changes in synaptic numbers after perinatal asphyxia in the rat, we investigated both neuronal and synaptic loss within particularly vulnerable areas, i.e. striatum, hippocampus and cerebellum. An antibody against synaptophysin, which is an intrinsic membrane protein located within smal $\rrbracket$ presynaptic vesicles of neurons, was used as a marker for presynaptic boutons [33,59]. Neuronal and synaptic loss was investigated using state-of-the-art quantitative methods considering stereologic principles.

\section{Methods}

\section{Induction of perinatal asphyxia}

Fifteen time-pregnant Wistar rats were purchased from Charles River Broekmans (The Netherlands). Rats were housed under standard conditions in conventional cages and given food (standard chow diet) and water ad libitum; lights on from 07.00 to $19.00 \mathrm{~h}$ in a temperature and humidity controlled room. The ethical board of the University of Maastricht (The Netherlands) approved animal care and all experimental procedures.

Global asphyxia at term (perinatal asphyxia) was induced as described previously [53]. Briefly, dams were decapitated immediately after delivery of one or two pups (control pups). Afterwards, dams were rapidly hysterectomized and the uterus horns containing the 
remaining pups were detached and placed in a water bath at $37^{\circ} \mathrm{C}$ for $20 \mathrm{~min}$ (time of perinatal asphyxia). Thereafter, pups were removed from the uterine horns, cleaned and stimulated to breatl by gently padding them on the chest. The mortality in the asphyxia group was $\pm 40-60 \%$. Both control and rats subjected to perinatal asphyxia were left to recover in an incubator at $37^{\circ} \mathrm{C}$ for $60 \mathrm{~min}$ and then randomly placed with a surrogate mother ( 10 pups per mother). At 21 days of age, the pups were weaned and placed separately in cages. It is important to note that these rats were not handled or subjected to any environmental changes before histologic analysis, as this is known to affect synaptic numbers. Only male rats were used for this study because motor and cognitive impairment was previously observed in male rats $[53,55]$.

\section{Perfusion and fixation}

Existing material from eleven rats (control group: $n=6$; asphyxia group: $n=5$ ) was used to study the total number of hippocampal pyramidal cells (using $100 \mu \mathrm{m}$ thick cresylviolet-stained sections). These animals were perfused at three weeks of age (as described in detail in [54]). Another twelve rats were perfused at two months of age (control: $\mathrm{n}=5$; asphyxia: $\mathrm{n}=7$ ) to investigate the total number of cerebellar Purkinje cells (using $16 \mu \mathrm{m}$ thick sections processed with immunohistochemistry) and the synaptic density within striatum, hippocampus and cerebellum (using $30 \mu \mathrm{m}$ thick sections also processed with immunohistochemistry). We used $16 \mu \mathrm{m}$ thick sections for analysis of the total number of Purkinje cells, since thick sections $(30 \mu \mathrm{m})$ were not suitable for quantification of the fluorescent marker (Cy3). Different sets of animals had to be used since it was not possible to realize the different section thicknesses on tissue from the same animals. Considering the general advice to keep numbers of laboratory animals as small as possible, it seemed appropriate to use the already existing material instead a new set of 11-12 animals at two months of age for the investigations of total numbers of neurons. All rats were anaesthetized with sodium pentobarbital $(60 \mathrm{mg} / \mathrm{kg}$; i.p.; Nembutal) and perfused transcardially ( $50 \mathrm{mmHg}$ ), first with tyrode, followed by $500 \mathrm{ml}$ fixative containing $4 \%$ paraformaldehyde and $2 \%$ picric acid in $0.1 \mathrm{M}$ phosphate buffer ( $\mathrm{PB}$; $\mathrm{pH} 7.6)$ at room temperature.

following perfusion, brains were removed from the skull and post fixed in $4 \%$ paraformaldehyde in $0.1 \mathrm{M} \mathrm{PB}$ at $4^{\circ} \mathrm{C}$ for 48 hrs or $2 \mathrm{~h}$ for investigation of the number of neurons or synapses, respectively. Thereafter, brain tissue was cryoprotected by immersion in $15 \%$ sucrose $/ 0.1 \mathrm{M}$ Tris buffered saline (TBS; $\mathrm{pH} 7.6$ ) for $48 \mathrm{~h}$ at $4^{\circ} \mathrm{C}$. The brain tissue was quickly frozen using $\mathrm{CO} 2$ snow and stored at $-80^{\circ} \mathrm{C}$ until further processing.

\section{Stereological analysis of total numbers of neurons and volumes of cell layers}

The forebrains of the three-week-old animals were entirely cut on a cryostat into serial 100 $\mu \mathrm{m}$-thick coronal sections. Every third section was stained with cresyl violet $(0.01 \%, 35 \mathrm{~min})$. The hippocampal pyramidal cell layer was delineated as described previously in [42]. No distinctions were made between the subregions, $\mathrm{CA} 1, \mathrm{CA} 2$ and $\mathrm{CA} 3$ within the hippocampus. Estimates of total numbers of cells were carried out under blind conditions by two independent 
investigators with the optical fractionator $[13,41,44]$. As the results obtained by these investigators were nearly the same, only one set of results is presented in this paper. The stereology workstation consisted of a modified light microscope (type BX50; Olympus), Olympus LPlanApo objectives ( $10 \times, \mathrm{NA}=0.40 ; 100 \times$, oil, $\mathrm{NA}=1.35$ ), a motorized specimen stage for automatic sampling (Maerzhaeuser, Wetzlar, Germany), an electronic microcator (Heidenhain, Traunreut, Germany), a CCD colour video camera (JAI, Glostrup, Denmark), a PC with framegrabber board and stereology software (C.A.S.T.-Grid, Olympus) and a $17^{\prime \prime}$ television screen monitor. All hippocampal pyramidal cells that came into focus within approximately 600 optical disectors systematically sampled were counted at a final magnification of $\times 3,600$. Disectors were $10 \mu \mathrm{m}$ high in an average actual section thickness of approximately $30 \mu \mathrm{m}$, measured using the microcator. The frame area was $492 \mu \mathrm{m}^{2}$. Estimated total numbers of cells were calculated from the number of counted neurons and the sampling probability $[13,41,44]$.

For investigating the total number of cerebellar Purkinje cells, the cerebelli of the two-months old animals were halved mediasagitally, and the left half of each animal was entirely cut into serial $16 \mu \mathrm{m}$-thick sagittal sections for investigation of the total number of cerebellar Purkinje cells. Every tenth section was incubated overnight with calbindin-D46 (1:10,000; P. Emson, Cambridge, UK) diluted in TBS with $0.3 \%$ Triton X-100. The primary antibody was visualized using biotinylated donkey anti-rabbit $\operatorname{IgG}(1: 800 ; 60 \mathrm{~min}$ at room temperature; Jackson Immunoresearch Laboratories, Inc., PA, USA) and streptavidin-Cy3 (1:2,000 dilution; 60 min at room temperature; Sigma). Between the incubation periods, the sections were washed with TBS and TBS-T. At the end of the staining procedure, sections were mounted with TBS: glycerol (1:3). All calbindin-immunoreactive cells were counted using an Olympus AX-70 microscope (magnification $\mathrm{x} 400$ ) and epifluorescent illumination. The total number of Purkinje cells per rat was estimated by multiplying the sum of immunoreactive cells in all sections by the sampling interval (i.e. equal to ten).

The volume of the hippocampal pyramidal cell layer (area CA1-3) as well as the volumes of the cerebellar molecular and granule cell layers were estimated as previously described [53]. Briefly, the region of interest was delineated and measured using the C.A.S.T Grid software at a final magnification of $\times 360$. From the obtained data and the corresponding average actual post-processing section thickness, total volumes were calculated by means of the Cavalieri's principle [14].

\section{Analysis of the density of synaptophysin-immunoreactive presynaptic boutons within striatum, hippocampus and cerebellum}

The immunohistochemical reactions for the analysis of the synaptophysin-immunoreactive presynaptic bouton (SIPB) density were performed simultaneously on all sections of all two-month-old animals using free-floating sections. For doing so, the forebrains were entirely cut on a cryostat into serial $30-\mu \mathrm{m}$-thick coronal sections, and the right cerebellar halves into serial $30 \mu \mathrm{m}$ thick sagittal sections. A monoclonal antibody against synaptophysin (Chemicon International, Inc., CA) was used for the analysis of synaptophysin-immunoreactive 
presynaptic bouton density (SIPB). TBS-T (0.1 M) was used for washing and diluting antibodies. Sections were pre-incubated in $5 \%$ normal goat serum (NGS; Sigma, The Netherlands) at room ternperature for $30 \mathrm{~min}$ and $2 \% \mathrm{NGS}$ was added to all solutions containing antibodies to minimize background staining. Sections were incubated with anti-synaptophysin serum ( $1: 2000)$ overnight at $4^{\circ} \mathrm{C}$. After washing with TBS and TBS-T, the tissue was immersed in goat antimouse IgG ( $1: 150$; American Qualex, San Clemente, CA., USA) for 1 h at room temperature, followed by a monoclonal mouse anti-peroxidase antibody $(1: 50$; Seralab, Crawley Down, Sussex, United Kingdom) with $5 \mathrm{mg} / \mathrm{ml}$ horseradish peroxidase (Sigma, type IV) added for h. After incubation with the antibodies and washing with TBS and TBS-T, all sections were incubated in $0.5 \% 3,3^{\circ}$-diaminobenzidine tetrahydrochloride (DAB; Sigma) containing $0.03 \%$ $\mathrm{H} 2 \mathrm{O} 2$ in TBS-T for 15 min at room temperature. All sections were washed twice with TBS and mounted on gelatine-coated glass slides. After air-drying, the sections were dehydrated in ascending concentrations of alcohol, cleared with microclair and coverslipped with Permount (Merck, Amsterdam, The Netherllands).

SIPB density analyses were carried out for the right brain half. Concerning striatum and hippocampus, investigations were performed on five to seven sections per brain region and animal, depending on the individual rostro-caudal extension of either striatum or hippocampus. On each section, the striatum was divided into four subregions, i.e., dorsal, medial, lateral, and ventral. Within the hippocampus, the analysis was focussed on the areas CA1 and CA3. For the cerrebellum, investigations were performed on approximately 10 sections per animal showing the mollecular layer.

Within each region of interest, two or three photomicrographs were taken and evaluated using an image analysis system, slightly modified for detection of greyscale punctae (AnalySIS -pro; Münster, Germany). The SIPB density was estimated as previously described [53,60]. Briefly, an Olympus BH -2 microscope equipped with a $100 \times$ oil immersion planachromatic objective and a CCD video camera (Paes, Zoeterwoude, The Netherlands) was used to obtain photomicrographs of the regions. Background levels were equalized and the detection threshold was tested and kept at the same level for all samples. The SIPB data are presented as the number of immunoreactive dots per $100 \mu^{2}$.

\section{Statistical analysis}

Mean and standard error of the mean ( $($ SEM) were calculated for all investigated variables. With respect to the SIPB density within the striatum, region-specific results were obtained for each animal (i.e. dorsal, medial, lateral, ventral) by calculating the mean of SIPB density within a specific region for all six sections (in rostral-caudal direction). In addition, orientationspecific results were obtained for each animal (i.e. rostral, medium, caudal) by calculating the mean of SIPB density per 2 sections. For example, from the first and second section in rostrocaudal direction all results were pooled and the mean of these data are presented. Finally, the mean SIPB density within the entire striatum was calculated by pooling all results of the different regions and sections. 
Group-specific means $( \pm \mathrm{SEM})$ were calculated for (i) the total number of hippocampal pyramidal cells, (ii) the volume of the hippocampal pyramidal cell layer, (iii) the volume of the cerebellar molecular and granule cell layer and (iiii) the SIPB densities within the investigated areas. Comparisons between the groups were performed with the Mann-Whitney U-test. Differences were considered statistically significant if $\mathrm{P}<0.05$. All calculations were performed using GraphPad Prism (Version 3.00 for Windows, GraphPad Software, San Diego, USA).

\section{Results}

\section{Total numbers of neurons and volumes of cell layers}

The results of the stereologic analysis are displayed in Figure 1. The mean total number of hippocampal pyramidal cells was $469,169 \pm 17,132$ for the control rats and $406,487 \pm 12,108$ for the asphyctic rats. There was a significant reduction in the estimated mean total number of hippocampal pyramidal cells in asphyctic rats compared to control rats $(-13.4 \% ; \mathrm{P}=0.02)$. With respect to the volume of the hippocampal pyramidal cell layer, there were no significant differences between the control and asphyctic rats $(-6.6 \% ; \mathrm{P}=0.33)$. The mean total number of cerebellar Purkinje cells was $126,693 \pm 18,583$ in control rats and $124,027 \pm 8,734$ in asphyctic rats at two months of age. There was no significant difference between the groups $(P=0.34)$. Furthermore, the mean volumes of both molecular and granule cell were unaffected by the asphyctic insult $(\mathrm{P}=0.20)$.

\section{Density of synaptophysin immunoreactive presynaptic boutons}

The results of the SIPB density measurements are shown in Figure 2 and 3 . When investigating the striatum in a subregion-specfic manner, the mean SIPB density was significantly reduced by $25.2 \%(\mathrm{P}=0.02)$ within the dorsal part of the striatum of asphyctic rats as compared to control rats. Furthermore, the mean SIPB density within the lateral, medial and ventral parts of the striatum was decreased $(-17.9 \%,-21.3 \%,-20.5 \%$ respectively). However, these differences did not reach statistical significance $(0.05<\mathrm{P}<0.10)$. When investigating the striatum in an orientation-specific manner, the mean SIPB density within the median and caudal part of the striatum was significantly reduced with $23.4 \%(P=0.002)$ and $27.5 \%(P=0.002)$ respectively. By contrast, the mean SIPB density within the rostral part of the striatum was not significantly reduced compared to control rats $(-13.6 \% ; \mathrm{P}=0.20)$. Within the entire striatum, SIPB density was reduced in asphyctic rats compared to control rats with $-22 \%(P=0.04)$. From these data it is reasonable to conclude that within the striatum the SIPB density was significantly reduced according to the asphyctic insult, predominantly in the dorsomedian and dorsocaudal parts of the striatum. 
Within the $C A 1$ and $C A 3$ regions of the hippocampus, the mean SIPB densities were not different between the groups. In consequence, the mean SIPB density within the entire hippocampus was unaffected by asphyctic insult (Figure 4).

With respect to the cerebellum, the mean SIPB density within the molecular layer of the asphyctic group was reduced by $19 \%$ compared to the control group. This difference was, however, not statistically significant $(\mathrm{P}=0.12)$. Most probably this was due to the large variability within the asphyctic group.

Hippocampus
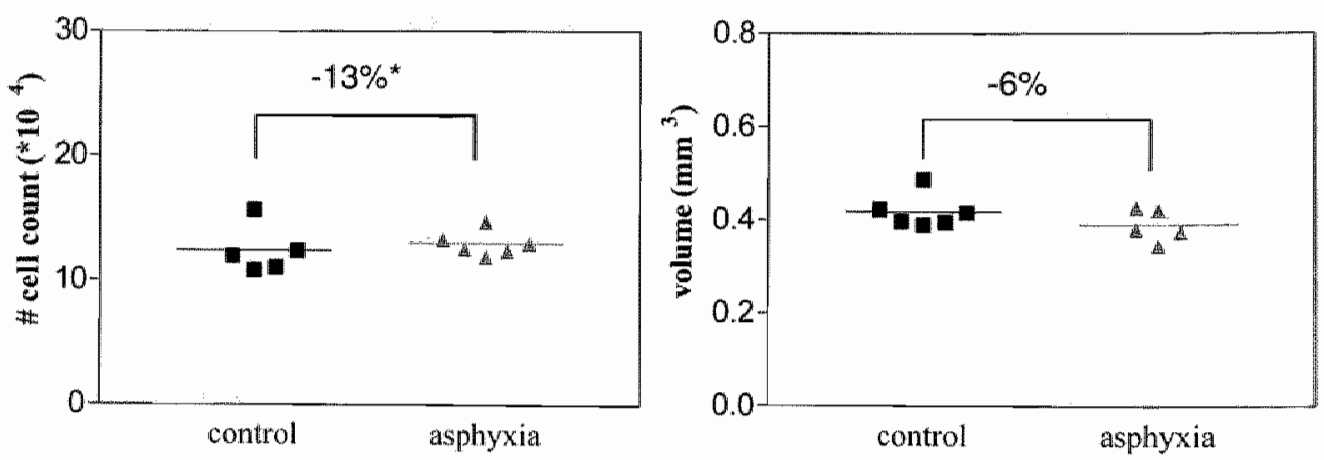

Cerebellum
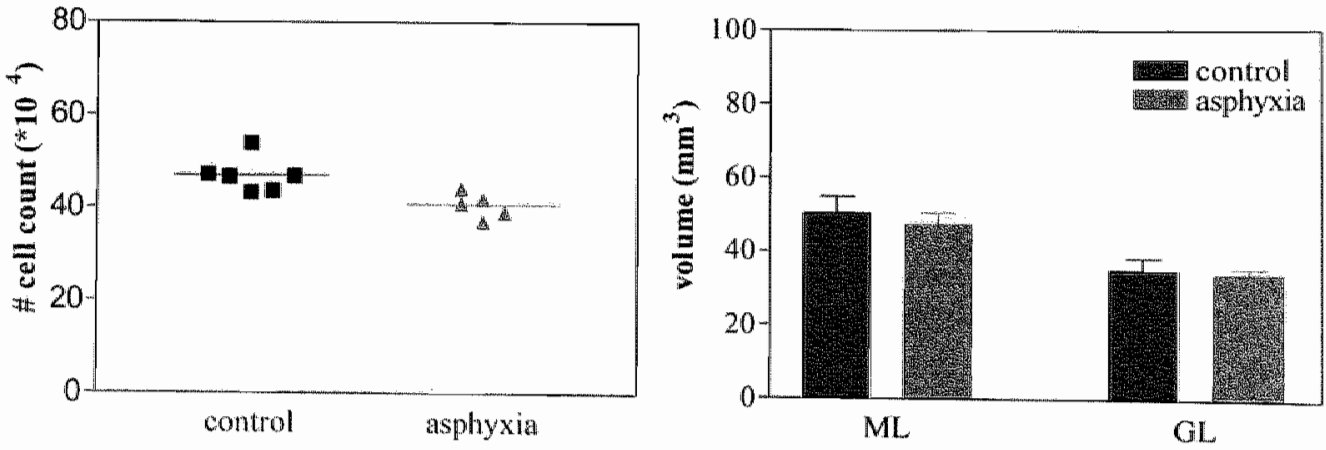

Figure 1. (A) Estimated total numbers of hippocampal pyramidal cells in CA1-3 of 3-weekold male Wistar rats. There was a significant decrease in the mean total number of pyramidal cells in asphyctic rats compared to control animals $(P=0.01)$. (B) Estimated volume of the hippocampal pyramidal cell layer (CA1-3) of 2 months old rats. There was no significant difference between asphyctic and control rats. (C) Estimated total numbers of Purkinje cells within the cerebellum. (D) Estimated volume of the molecular layer (ML) and granule layer (GL) within the cerebellum. Perinatal asphyxia did not affect the mean number of Purkinje cells or volume of the ML and GL. * $P<0.05$ 
Since the volumes of the pyramidal cell layer within the hippocampus, the entire striatum, and the molecular and granule cell layers within cerebellum were unaffected by the asphyctic insult, it was reasonable to interpret the SIPB data as representing estimates of the numbers of presynaptic boutons within the different areas (cf [53]). It was therefore reasonable to conclude that the perinatal asphyxia regimen investigated here reduced the number of synaptophysin-immunoreactive presynaptic boutons within the striatum, but not within the hippocampus at two months of age. With respect to the cerebellum, it has to be noted that there is a tendency toward a decrease in total number of presynaptic boutons in the cerebellum in asphyctic rats.

\section{Discussion}

Recently it was proposed that a deficiency in the number of synapses at birth might trigger the premature onset of neurodegenerative diseases, such as dementia, or induce a more rapid progression of such diseases [50]. In the present study, we show that perinatal asphyxia in the rat affects both neuronal and synaptic numbers in a region-specific manner. Concerning the SIPB density, the dorsomedian and dorsocaudal parts of the striatum were affected by the asphyctic insult. Within the hippocampus, the number of pyramidal neurons within the CA1$\mathrm{CA} 3$ region was reduced in asphyctic rats at 21 days, whereas the mean number of SIPB was
unchanged at 2 months of age. Finally, no evidence for Purkinje cell loss or loss of SIPB within the cerebellum could be found at two months of age, suggesting that the cerebellum was relatively spared during the insult.

This is the first study showing that perinatal asphyxia affects synaptic numbers in a regionspecific manner. This may be relevant since perinatal asphyxia could increase the risk for developing neurodegenerative diseases in the adult.

\section{Striatum}

The striatum is one of the most severely damaged brain areas during an asphyxial insult. The data provided in the present study show that perinatal asphyxia leads to a reduction of the mean total number of SIPB within the dorsomedian and dorsocaudal part of the striatum. The regions of the striatum (dorsal, lateral ventral and medial) described in this study are part of the caudate-putamen complex $(\mathrm{CPu})$, excluding the nucleus accumbens. The significance of the loss of SIPB within the different subregions of the striatum depends on which synapses disappear. There are several possibilities: (i) the loss of $\mathrm{SIPB}$ may reflect neuronal death within the striatum or (ii) a loss in synaptic input from the substantia nigra, cortical subregions and/or the thalamus. Furthermore, (iii) the loss of SIPB may be the result of impaired synaptogenesis or impaired rearrangement of existing/new synapses during postnatal development following asphyxia. With respect to the first possibility, we have previously shown that perinatal asphyxia in the rat leads to a $16 \%$ reduction in striatal neurons at three weeks of age [54]. 
Striatum
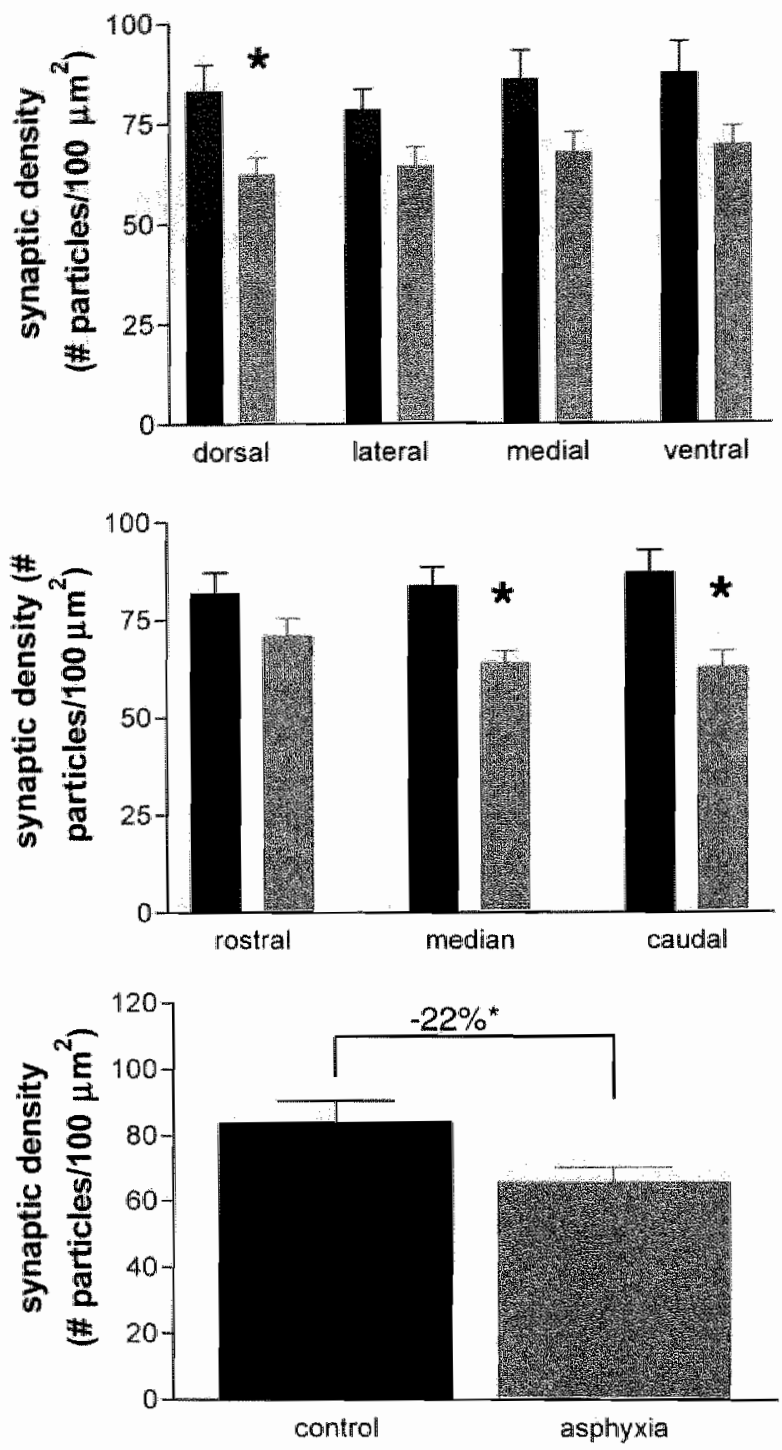

control

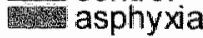

Figure 2. Densities of synaptophysin-immunoreactive presynaptic boutons (SIPB) within the striatum of asphyctic or control rats. (A) Subregion-specific SIPB densities (i.e. dorsal lateral, medial, and ventral part of the striatum). SIPB density was significantly reduced in the dorsal part of the striatum of asphyctic rats compared to control rats $(P=0.02) .(B)$ Orientation-specific SIPB densities (i.e. rostral, median and caudal part of the striatum). SIPB density in asphyctic rats was reduced in the median $(-23.4 \% ; P=0.002)$ and caudal $(-27.5 \% ; P=0.002)$ parts. (C) SIPB densities within the entire striatum. Perinatal asphyxia reduced the SIPB density with $22 \%(P=0.04) .{ }^{*} P<0.05 ;{ }^{* *} P<0.01$. 
Hippocampus
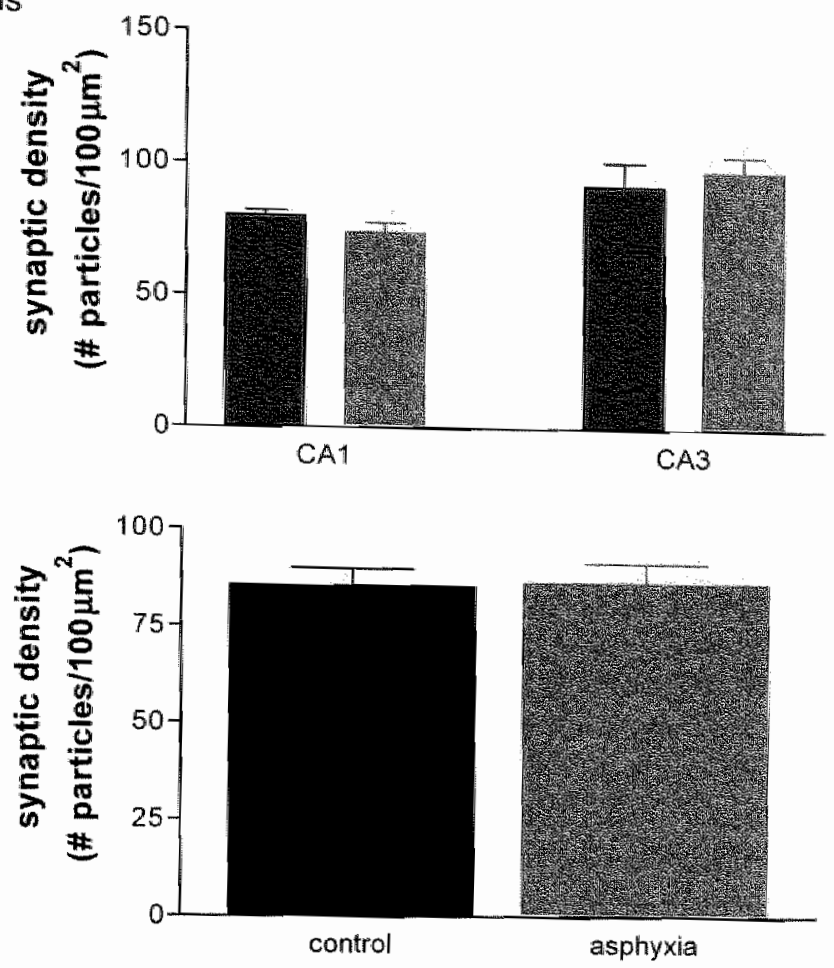

Cerebellum

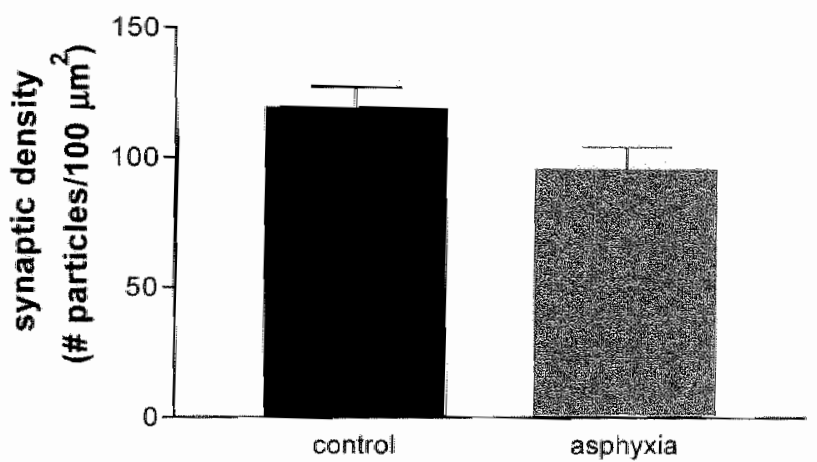

Figure 3. (A) SIPB densities within CA1 and CA3 region of the hippocampus of 2-monthold control and asphyctic rats. (B) SIPB densities within the entire pyramidal layer of the hippocampus (mean CA1 and CA3). (C) SIPB densities within the molecular layer of the cerebeilum at 2 months of age. There were no differences between control and asphyctic rats within any hippocampal subregion or the cerebellar molecular layer. 
Further, perinatal asphyxia particularly affects GABAergic projection neurons as well as GABAergic and cholinergic intemeurons within the striatum $[27,55]$. Hence, loss of SIPB within the dorsomedian and dorsocaudal parts of the striatum may reflect the loss of these neurons. Concerning the second possibility ( $i_{*} e_{*}$ loss of SIPB within the striatum due to loss of synaptic inpur from the substantia nigra pars compacta ( $\mathrm{SNC}$ ), thalamus and/or cortical areas) one has to consider that the main targets of the corticostriatal terminals are the spines of the GABAergic medium spiny projection neurons and the GABAergic interneurons expressing the calcium-binding protein, parvalbumin (PV3) $[38,48]$. The GABAergic interneurons as well as other interneurons, such as the cholinergic ones, distribute their axons within the striatum and make synaptic contacts with the spiny projection neurons. Dopaminergic terminals make direct synaptic contacts with spiny projections neurons [22]. It was shown that perinatal asphyxia results in a decrease of striatal dopamine release [26]. Hence, loss of SIPB within the CPu might be related to a reduced nigrostriatal dopamine input after perinatal asphyxia. 6-OHDA lesions of the nigrostriatal dopamine system involving the dorsomedial part of the CPu affect both locomotion and drug-induced turning behaviour, whereas $l$ esions involving the ventrolateral parts of the CPu impair movement initiation, sensorimotor orientation and skilled motor behaviour [20]. Thus, loss of SIPB within the dorsal parts of the striatum might therefore be related to the decrease in motor activity described after perinatal asphyxia in rats [55]. By contrast, functional consequences related to synaptic loss within the median-caudal part of the CPu are not known. Finally, with respect to the possibility that asphyxia-induced loss of SIPB may be the result of impaired synaptogenesis or impaired rearrangement of existing/new synapses, it should be mentioned that perinatal asphyxia may change endogenous growth factor levels during postnatal development. Several studies have shown that growth factors were both up-and downregulated after brain injury and can directly affect both neuro- and synaptogenesis $[1,6,34]$. Hence, perinatal asphyxia might lead to the re-arrangement of synapses or: to a decrease in synaptogenesis.

Previously, we have shown that the perinatal asphyxia paradigm investigated in the present study results in long-term cognitive impairment without alteration in SIPB density in the rat striatum at 22 months of age [53]. The data presented in the present study seem to contradict these results. However, the difference in results might reflect changes in SIPB during development or aging. Synaptophysin-immunoreactivity within the striatum peaks at two months of age in the rat and continues to decline afterwards [47]. Therefore, we speculate that perinatal asphyxia affects the decline in the number of SIPB during aging and consequently the total number of SIPB in later life. However, it is important to keep in mind that behavioural tasks, such as the Morris water maze, can also influence the number of synapses (and, therefore, the number of SIPB). Many studies have shown that learning and physical activity can induce synaptogenesis within the developing and aging brain [10]. Based on these data, it is reasonable to assume that the mean numbers of SIPB seen at 22 months of age were affected by behavioural testing, which was carried out at 6 weeks and 18 months of age [53]. Further research should be conducted to establish whether loss of SIPB after perinatal asphyxia is reversible by learning or physical activity, and whether this can be used as a therapeutic approach. 


\section{Hippocampus}

In the present study, it was shown that perinatal asphyxia leads to neuronal loss $(-13 \%)$ within the pyramidal cell layer of the hippocampus at 3 weeks of age. Although we used state-of-theart stereology to assess the number of pyramidal cells within the rat hippocampus, we found less neurons within the hippocampus (approximately 470,000) of control rats than previously reported in the literature (i.e., approximately $600,000-700,000 ;[43,58]$ ). However, this difference was far less than the previously reported differences in neuronal numbers by factors of about 3 or even more [12]. Our data concerning hippocampal pyramidal cell loss after perinatal asphyxia are in agreement with results of previous studies showing cell death of hippocampal cells after hypoxic-ischemic or asphyctic insults $[8,32,46]$. Perinatal asphyxia might directly damage neurons within the hippocampus and consequently reduce the number of neurons in later life.

Interestingly, revently evidence was provided for an increase in BDNF protein levels within the hippocampus after perinatal asphyxia (using the same animal model) during postnatal development [40]. Several studies have shown that BDNF is able to promote neurogenesis as wel] as synaptogenesis and plays a major role during recovery from brain injury $[23,34,52]$. Thus, a transient increase in hippocampal BDNF protein levels may explain why the numbers of SIPB within the CA1 and/or CA3 regions of the hippocampus were unaffected by the perinatal asphyctic insult at two months of age. However, the lack of effect on SIPB numbers within the hippocampus might also be explained by the severity of the perinatal asphyctic insult. In the present study a perinatal asphyxia paradigm was used that induces very subtle functional deficits $[16,53,55]$. Moreover, this perinatal asphyxia paradigm leads to a small decrease in the number of hippocampal pyramidal cells $(-13.3 \%)$ during a period in which proliferation within the dentate gyrus and synaptogenesis within the hippocampus continues at high levels $[3,36]$. The granule cell axons (i.e, the mossy fibers) of the dentate gyrus project to the CA3 pyramidal cells, which in turn give rise to axons that innervate the CAI field as well as the CA3 field itself [49]. The SIPB number within the CA1 and CA3 fields were unaffected by the perinatal asphyxial insult, suggesting that intrahippocampal synaptic circuits were resistant to the asphyctic insult or that the synaptic circuits were rearranged after the asphyctic insult, i.e. yielding more synapses per neuron. Consistent with our results, behavioural studies investigating cognitive performance after perinatal asphyxia have not provided evidence for major changes in hippocampal function during the first two months of life $[4,25]$.

\section{Cerebellum}

The cerebellum plays an important role in motor performance and, according to recent studies, it is also involved in regulating cognitive interactions $[5,31]$. It receives input from several cortical areas, from the hypothalamus, and from serotonergic and dopaminergic nuclei within the brain stem. Its output is focused on specific componens of the motor system, such as the primary cortex and the premotor cortex. Purkinje cells within the cerebellum use GABA as transmitter and exert postsynaptic inhibitory effects on cells of the cerebellar and vestibular 
nuclei $[18,35]$. The molecular layer of the cerebellum contains the dendritic arbors of the Purkinje cells and Bergmann glial fibers, of which axons terminate on the primary dendrites of Purkinje cells [56]. The results presented here show that there were no significant changes in the number of Purkinje cells and SIPB density within the molecular layer of the cerebellum induced by asphyxia. The volumes of the molecular and granule cell layers were unchanged after perinatal asphyxia, suggesting that no plastic changes occurred.

Our data confirm results from a previous study investigating morphologic changes in the cerebellum of 2-year-old rats following perinatal asphyxia, in which neither long-lasting changes in the mean number of Purkinje cells or the volumes of both molecular and granule cell layers were found [57]. Furthermore, our results are in agreement with previous studies reporting approximately $100,000-150,000$ Purkinje cells within the cerebellum of the rat [43].

Interestingly, there was a tendency towards a decrease in SIPB numbers within the molecular layer of the cerebellum of asphyctic rats compared to control rats. This reduction in SIPB numbers might result in a reduction in behavioural performance. Behavioural deficits after perinatal asphyxia that are known to depend on cerebellar function have been documented, including tests of balance/walking patterns and co-ordinated motor performance $[4,25,55]$. However, motor deficits occurring after perinatal asphyxia are not consistent [16] and performance might have been affected by the behavioural tasks itself [21]. To investigate the large variability in SIPB density and behavioural performance and the consequences of SIPB loss within the cerebellum, structural changes within the cerebellum should be correlated to behavioural performance in a next study.

\section{Conclusions}

In summary, perinatal asphyxia reduced SIPB density within the dorsal and median-caudal striatum, whereas the SIPB densities within the hippocampal pyramidal cell layer was relatively spared. In addition, there was slight reduction in SIPB density within the cerebellum. The mean number of hippocampal pyramidal cells was reduced after asphyxia. By contrast, the mean number of cerebellar Purkinje cells was unaffected by the asphyctic result. We therefore conclude that perinatal asphyxia causes region-specific damage primarily within the rat forebrain. Since both the striatum and cerebellum play a key role in motor control, loss of SIPB within these regions might explain the motor deficits induced by perinatal asphyxia. 


\section{References}

[1] B. Alsina, T. Vu and S. Cohen-Cory. 2001. Visualizing symapse formation in arborizing optic axons in vivo: dynamics and modulation by BDNF. Nat. Neurosci. 4: 1093-101.

[2] W. Balduini, V. De Angelis, E. Mazzoni and M. Cimino. 2000. Long-lasting behavioral alterations following a hypoxic/ischemic brain injury in neonatal rats. Brain Res. 859: 318-25.

[3] S.A. Bayer. 1980. Development of the hippocampal region in the rat. II. Morphogenesis during embryonic and early postnatal life. J. Comp. Neurol. 190: 115-34.

[4] P. Boksa, A. Krishnamurthy and W. Birooks. 1995. Effects of a period of asphyxia during birth on spatial learning in the rat. Pediatr. Res. 37: 489-96.

[5] L.R. Caplan. 2002. The cerebellum may be directly involved in cognitive functions. Neurology 59: $790-1$.

[6] C.W. Cotman and N.C. Berchtold. 2002. Exercise: a behavioral intervention to enhance brain health and plasticity. Trends Neurosci. 25: 295-301.

[7] C.W. Cotman and M. Nieto-Sampedro. 1984. Cell biology of synaptic plasticity. Science 225: $1287-94$.

[8] E. Dell'Anna, Y. Chen, E. Engidawork, K. Andersson, G. Lubec, J. Luthman and M. HerreraMarschitz. 1997. Delayed neuronal death following perinatal asphyxia in rat. Exp. Brain Res. 115: 105-15.

[9] M.K. Floeter and W.T. Greenough. 1979. Cerebellar plasticity: modification of Purkinje cell structure by differential rearing in monkeys. Science 206: 227-9.

[10] Y. Geinisman, R.W. Berry, J.F. Disterhoft, J.M. Power and E.A. Van der Zee. 2001. Associative learning elicits the formation of multiple-synapse boutons. J. Neurosci. 21: 5568-73.

[11] E. Gould, P. Tanapat, N.B. Hastings and T.J. Shors. 1999. Neurogenesis in adulthood: a possible role in learning. Trends Cogn. Sci. 3: 186-192.

[12] R.W. Guillery and K. Herrup. 1997. Quantification without pontification: choosing a method for counting objects in sectioned tissues. J. Comp. Neurol. 386: 2-7.

[13] H.J. Gundersen, P. Bagger, T.F. Bendtsen, S.M. Evans, L. Korbo, N. Marcussen, A. Moller, K. Nielsen, J.R. Nyengaard, B. Pakkenberg and et al. 1988. The new stereological tools: disector, fractionator, nucleator and point sampled intercepts and their use in pathological research and diagnosis. Apmis 96: 857-81.

[14] H.J. Gundersen and E.B. Jensen. 1985. Stereological estimation of the volume-weighted mean volume of arbitrary particles observed on random sections. J. Microsc. 138: 127-42.

[15] N.B. Hastings and E. Gould. 1999. Rapid extension of axons into the CA3 region by adultgienerated granule cells. J. Comp. Neurol. 413: 146-54.

[16] H. Hoeger, M. Engelmann, G. Bernert, R. Seidl, H. Bubna-Littitz, W. Mosgoeller, B. Lubec and $G$. Lubec. 2000 . Long term neurological and behavioral effects of graded perinatal asphyxia in the rat. Life Sci. 66: 947-62.

[17] H. Ishimaru, F. Casamenti, K. Ueda, Y. Maruyama and G. Pepeu. 2001. Changes in presynaptic proteins, SNAP-25 and synaptophysin, in the hippocampal CA1 area in ischemic gerbils. Brain Res. 903: 94-101.

[18] M. Ito and M. Yoshida. 1964. The cerebellar-evoked monosynaptic inhibition of Deiters" neurones. Experientia 20: 515-6.

[19] C.D. Katsetos, E. Spandou, A. Legido, M.L. Taylor, S.A. Zanelli, J.P. de Chadarevian, S. Christakos, O.P. Mishra and M. Delivoria-Papadopoulos. 2001. Acute hypoxia-induced alterations of calbindin-D28k immunoreactivity in cerebellar Purkinje cells of the guinea pig fetus at term. J. Neuropathol. Exp. Neurol. 60: 470-82.

[20] D. Kirik, C. Rosenblad and A. Bjorklund, 1998. Characterization of behavioral and neurodegenerative changes following partial lesions of the nigrostriatal dopamine system 
induced by intrastriatal 6-hydroxydopamine in the rat. Exp. Neurol. 152: 259-77.

[21] A.Y. Klintsova, C. Scamra, M. Hoffman, R.M. Napper, C.R. Goodlett and W.T. Greenough. 2002. Therapeutic effects of complex motor training on motor performance deficits induced by neonatal binge-like alcohol exposure in rats: II. A quantitative stereological study of synaptic plasticity in female rat cerebellum. Brain Res. 937: 83-93.

[22] Y. Kubota, S. Inagaki, S. Kito and J.Y.Wu. 1987. Dopaminergic axons directly make synapses with GABAergic neurons in the rat neostriatum. Brain Res. 406: 147-56.

[23] J. Lee, W. Duan and M.P. Mattson. 2002. Evidence that brain-derived neurotrophic factor is required for basal neurogenesis and mediates, in part, the enhancement of neurogenesis by dietary restriction in the hippocampus of adult mice. J. Neurochem. 82: 1367-75.

[24] A. Legido, Parikh, N.A., Taylor, M.L., Kaleyias, J.K., de Chadarevian, J., Katsetos, C.D. 2002. Decreased immunoreactivity of Calbindin-D28K in cerebellar Purkinje cells of children with hypoxic/ischemic encephalopathy. Pediatric Academic Societies. Abstract nr. 2603.

[25] C.F. Loidl, A.W. Gavilanes, E.H. Van Dijk, W. Vreuls, A. Blokland, J.S. Vles, H.W. Steinbusch and C.E. Blanco. 2000. Effects of hypothermia and gender on survival and behavior after perinatal asphyxia in rats. Physiol. Behav. 68: 263-9.

[26] C.F. Loidl, M. Herrera-Marschitz, K. Andersson, Z.B. You, M. Goiny, W.T. O'Connor, R. Silveira, R. Rawal, B. Bjelke, Y. Chen and el al. 1994. Long-term effects of perinatal asphyxia on basal ganglia neurotransmitter systems studied with microdialysis in rat. Neurosci. Lett. 175: $9-12$

[27] E.C. Mallard ${ }_{4}$ H.J. Waldvogel, C.E. Williams, R.L. Faull and P.D. Gluckman. 1995. Repeated asphyxia causes loss of striatal projection neurons in the fetal sheep brain. Neuroscience 65:827-36.

[28] G. Martinez, C. Di Giacomo, M.L. Carnazza, V. Sorrenti, R. Castana, M.L. Barcellona, J.R. Perez-Polo and A. Vanella. 1997. MAP2, synaptophysin immunostaining in rat brain and behavioral modifications after cerebral postischemic reperfusion. Dev. Neurosci. 19: 45764.

[29] E. Masliah. M. Mallory, L. Hansen, R. DeTeresa and R.D. Terry. 1993. Quantitative synaptic alterations in the human neocortex during normal aging. Neurology 43: 192-7.

[30] C. Mazer, J. Muneyyirci, K. Taheny, N. Raio, A. Borella and P. Whitaker-Azmitia. 1997. Serotonin depletion during synaptogenesis leads to decreased synaptic density and learning deficits in the adult rat: a possible model of neurodevelopmental disorders with cognitive deficits. Brain Res. 760:68-73.

[31] F.A. Middleton and P.L. Strick. 2000. Basal ganglia and cerebellar loops: motor and cognitive circuits. Brain Res. Birain Res. Rev. 31: 236-50.

[32] W. Nakajima, A. Ishida, M.S. Lange, K.L. Gabrieison, M.A. Wilson, L.J. Martin, M.E. Bllue and M.V. Johnston. 2000. Apoptosis has a prolonged role in the neurodegeneration after hypoxic ischemia in the newborn rat. $J$. Neurosci. 20: 7994-8004.

[33] F. Navone, R. Jahn, G. Di Gioia, H. Stukenbrok, P. Greengard and P. De Camilli. 1986. Protein p38: an integral membrane protein specific for small vesicles of neurons and neuroendocrine cells. J. Cell Biol. 103: 2511-27.

[34] M. Nieto-Sampedro, E.R. Lewis, C.W. Cotman, M. Manthorpe, S.D. Skaper, G. Barbin, F.M. Longo and S. Varon. 1982. Brain injury causes a time-dependent increase in neuronotrophic activity at the lesion site. Science 217: 860-1.

[35] K. Obata. 1969. Gamma-aminobutyric acid in Purkinje cells and motoneurones. Experientia 25: 1283.

[36] D.A. Peterson. 2002. Stem cells in brain plasticity and repair. Curr. Opin. Pharmacol. 2: 3442.

[37] J.J. Pysh and G.M. Weiss. 1979. Exercise during development induces an increase in Purkinje cell dendritic tree size. Science 206: 230-2. 
[38] S. Ramanathan, J.J. Hanley, J.M. Deniau and J.P. Bolam. 2002. Synaptic convergence of motor and somatosensory cortical afferents onto GABAergic interneurons in the rat striatum. J. Neurosci. 22: 8158-69.

[39] V. Ramirez-Amaya, M.L. Escobar, V. Chao and F. Bermudez-Rattoni. 1999. Synaptogenesis of mossy fibers induced by spatial water maze overtraining. Hippocampus 9: 631-6.

[40] A. Scheepens, Wassink, G., Blanco, C.E. in press. The effect of a global birth asphyxia on the ontogeny of BDNF and NGF protein expression in the brain. Dev. Brain Res.

[41] C. Schmitz. 1998. Variation of fractionator estimates and its prediction. Anat. Embryol. (Berl) 198: 371-97.

[42] C. Schmitz, E. Bultmann, M. Gube and H. Korr. 1999. Neuron loss in the mouse hippocampus following prenatal injection of tritiated thymidine or saline. Int. J. Dev. Neuroscl. 17: 185-90.

[43] C. Schmitz, M. Dafotakis, H. Heinsen, K. Mugrauer, A. Niesel, G.J. Popken, M. Stephan, W.D. Van de Berg, S. von Horsten and H. Korr. 2000. Use of cryostat sections from snapfrozen nervous tissue for combining stereological estimates with histological, cellular, or molecular analyses on adjacent sections. J. Chem. Neuroanat. 20: 21-9.

[44] C. Schmitz and P.R. Hof. 2000. Recommendations for straightforward and rigorous methods of counting neurons based on a computer simulation approach. J. Chem. Neuroanat. 20: 93-114.

[45] L.M. Schrott. 1997. Effect of training and environment on brain morphology and behaviour. Acta Paediatr. Suppl. 422: 45-7.

[46] R.A. Sheldon, J.J. Hall, L.J. Noble and D.M. Ferriero. 2001. Delayed cell death in neonatal mouse hippocampus from hypoxia-ischemia is neither apoptotic nor necrotic. Neurosci. Lett. 304: 165-8.

[47] S. Shimohama, S. Fujimoto, Y. Sumida, K. Akagawa, T. Shirao, Y. Matsuoka and T. Taniguchi. 1998. Differential expression of rat brain synaptic proteins in development and aging. Biochem. Biophys. Res. Commun. 251: 394-8.

[48] Y. Smith, B.D. Bennett, J.P. Bolam, A. Parent and A.F. Sadikot. 1994. Synaptic relationships between dopaminergic afferents and cortical or thalamic input in the sensorimotor territory of the striatum in monkey. J. Comp. Neurol. 344: 1-19.

[49] L.W Swanson, C. Kohler, A. Bjorklund. 1987. The limbic region. I: The septohippocampal system. In: A. Bjorklund, T. Hokfelt, L.W. Swanson. Handbook of chemical Neuroanatomy: Integrated systems of the CNS, Part I. Elsevier, the Netherlands 5: 125-277.

[50] R.D. Terry and R. Katzman. 2001. Life span and synapses: will there be a primary senile dementia? Neurobiol. Aging 22: 347-8; discussion 353-4.

[51] U.I. Tuor, T.J. Hudzik, K. Malisza, S. Sydserff, P. Kozlowski and M.R. Del Bigio. 2001. Longterm deficits following cerebral hypoxia-ischemia in four-week-old rats: correspondence between behavioral, histological, and magnetic resonance imaging assessments. Exp. Neurol. 167: 272-81.

[52] W.J. Tyler and L.D. Pozzo-Miller. 2001. BDNF enhances quantal neurotransmitter release and increases the number of docked vesicles at the active zones of hippocampal excitatory synapses. J. Neurosci. 21: 4249-58.

[53] W.D. Van de Berg, A. Blokland, A.C. Cuello, C. Schmitz, W. Vreuls, H.W. Steinbusch and C.E. Blanco. 2000. Perinatal asphyxia results in changes in presynaptic bouton number in striatum and cerebral cortex-a stereological and behavioral analysis. J. Chem. Neuroanat. 20: $71-82$.

[54] W.D. Van de Berg, C. Schmitz, H.W. Steinbusch and C.E. Blanco. 2002. Perinatal asphyxia induced neuronal loss by apoptosis in the neonatal rat striatum: a combined TUNEL and stereological study. Exp Neurol 174: 29-36. 
[55] W.D. Van de Berg, M. Kwailtaal, A.J.A. De Louw, N.P.A. Lissone, C. Schmitz, R.L.M. Faull, A. Blokland, C.E. Blanco, H.W.M. Steinbusch. 2003. Impact on perinatal asphyxia on the GABAergic and locomotor system. Neuroscience 117: 83-96.

[56] J.Voogd D Jaarsma, E Marani. 1996. The cerebellum: chemoarchitecture and anatomy. In L.W. Swanson, A. Bjorklund, T. Hokfelt. Handbook of chemical neuroanatomy: Integrated systems of the CNS, Part III. Elsevier, The Netherlands 12: 1-369.

[57] R. Weitzdoerfer, N. Gerstl, H. Hoeger, W. Mosgoeller, W. Dreher, E. Engidawork, J. Overgaard-Larsen and B. Lubec. 2002. Long-term sequelae of perinatal asphyxia in the aging rat Cell. Mol Lite Sci. 59:519-26.

[58] M.J. West, L. Slomianka and H.J. Gundersen. 1991. Unbiased stereological estimation of the total number of neurons in thesubdivisions of the rat hippocampus using the optical fractionator. Anat. Rec. 231: $482-97$.

[59] B. Wiedenmannand W.W. Franke, 1985. Identification and localization of synaptophysin, an integral membrane glycoprotein of Mr 38,000 characteristic of presynaptic vesicles. Cell 41:1017-28.

[60] T.P. Wong, P.M. Campbell, A. Ribeiro-da-Silva and A.C. Cuello. 1998. Synaptic numbers across cortical laminae and cognitive performance of the rat during ageing. Neuroscience: 84: 403-12. 


\section{Perinatal asphyxia results in}

changes in presynaptic bouton number in striatum and cerebral cortex

\section{A stereological and behavioural analysis}

WDJ van de Berg, A Blokland, AC Cuello, C Schmitz, WVreuls, HWM Steinbusch, CE Blanco

J. Chem. Neuroanatomy 2000;20:71-82 


\section{Abstract}

Deficits in cognitive function have been related to quantitative changes in synaptic population, particularly in the cerebral cortex. Here, we used an established model of perinatal asphyxia that induces morphological changes, i.e. neuron loss in the cerebral cortex and striatum, as well as behavioural deficits. We hypothesized that perinatal asphyxia may lead to a neurodegenerative process resulting in cognitive impairment and altered presynaptic bouton numbers in adult rats. We studied cognitive performance at 18 months and presynaptic bouton numbers at 22 months following perinatal asphyxia. Data of the spatial Morris water escape task did not reveal clear memory or learning deficits in aged asphyctic rats compared to aged control rats. However, a memory impairment in aged rats versus young rats was observed, which was more pronounced in asphyctic rats. We found an increase in presynaptic bouton density in the parietal cortex, whereas no changes were found in striatum and frontal cortex in asphyctic rats. An increase of striatal volume was observed in asphyctic rats, leading an increase in presynaptic bouton numbers in this area. These findings stress the issue that volume measurements have to be taken into account when determining presynaptic bouton density. Furthermore, perinatal asphyxia led to region-specific changes in presynaptic bouton numbers and it worsened the age-related cognitive impairment. These results suggest that perinatal asphyxia induced neuronal loss, which is compensated for by an increase in presynaptic bouton numbers. 


\section{Introduction}

Perinatal asphyxia causes irreversible damage to the brain leading to neuron loss in certain brain regions such as the cerebral cortex and the striatum $[10,27,41]$. Furthermore, perinatal asphyxia can produce several long-term neurological and behavioural changes, such as reduced learning disabilites, mental retardation, spasticity and epilepsy $[37,43,48]$. Epidemiological studies have documented an increased incidence of birth complications among patients who developed psychiatric disorders $[9,23]$ see for recent reviews [33,34]. In particular, a strong association has been found between perinatal brain damage and the age of onset of schizophrenia $[25,42]$.

Only few studies have examined the long-term effect of perinatal asphyxia on behaviour in animal models. Main findings of these studies were that perinatal asphyxia led to learning deficits [4], reduced anxiety-related behavior [22] and reduced locomotor activity [29] at 3-5 months of age.

Deficits in cognitive and motor function have been associated with alterations in synaptic numbers and organization, particularly in the cerebral cortex and striatum. Several papers have reported a loss of synaptic input to the cortex during aging and related this to a decline in cognitive function $[32,47]$. On the other hand, no changes in synaptic numbers have been reported in the hippocampus during aging $[6,36]$. Increased synaptic numbers in the cerebral cortex have been reported as a consequence of cerebral ischemia [40] or associative learning [3].

Several methods have been used for estimating the synaptic numbers i.e. quantitative lightmicroscopical or ultrastructural analyses of synaptic densities or stereology $[7,18,24,47]$. Quantification of synapses at the ultrastructural level is based on the visualization of individual synaptic contacts. Quantification of synapses at the light-microscopical level is based upon the detection of immunostained particles using an image analysis, applying synaptophysin as a specific and selective marker for presynaptic boutons $[46,47]$. This method allows quantification of presynaptic bouton density, which is a ratio that represents the total number of presynaptic boutons $(\mathrm{N})$, divided by the volume of the structure (Vref). Stereological methods for estimating presynaptic bouton numbers at the light-microscopic level are based on the quantification of synaptophysin-stained presynaptic boutons at a high magnification in 3D-space.

In this study, we present a new approach for the analysis of presynaptic bouton numbers, combining light-microscopic analysis of presynaptic bouton density [47] with volume estimates using the point-counting method and the Cavalieri's principle [19]. This combination of these two values is referred to as the presynaptic bouton numbers. We tested whether neurodegeneration during development induced by perinatal asphyxia lead to changes presynaptic bouton numbers in adulthood and whether these changes were related to behavioural changes, i.e. impaired cognition. 


\section{Materials and methods}

\section{Animals}

Full-term pregnant Wistar rats were used either as surrogate mothers $(n=13)$ or as experimental group $(\mathrm{n}=34)$. Dams were purchased from Charles River-Broekmans (Someren, The Netherlands). They were housed in the Animal Care Department of Maastricht University under standard conditions $\left(12: 12 \mathrm{~h}\right.$ light: dark cycles, $20^{\circ} \mathrm{C}$, with free access to standard laboratory chow and water). Animal care and procedures were approved by the ethical board of University of Maastricht.

\section{Induction of perinatal asphyxia and experimental set-up}

A rat model of perinatal asphyxia was applied as described previously by Loidl et al. $[29,30]$. Briefly, dams from the experimental group were decapitated immediately after one or two pups were delivered vaginally (control vaginal delivery; CVD). Afterwards, they were rapidly hysterectomized, the uterus horns containing the remaining foetuses were detached and the horns were placed in a water bath at $37^{\circ} \mathrm{C}$ for $20 \mathrm{~min}$ (severe perinatal asphyxia; SPA). After 20 min in the water bath, pups were removed from the uterus horns and stimulated to breath by drying up the skin and gently padding them on the chest. The maximal duration of the resuscitation was 5 min. If a pup was not able to gasp within this period, it was considered dead and excluded from the study. These procedures were performed under a heating lamp. The pups were left to recover for 60 min under the heating lamp, marked and then placed with a surrogate mother. Each surrogate mother received 8 pups, which were randomly selected from the CVD and SPA group. The percentage of mortality in the SPA group was approximately $80 \%$. All pups belonging to the CVD group were breathing immediately after birth and all survived.

At postnatal day (P) 21 , the animals were weaned and housed in Makrolon ${ }^{\text {MM }}$ plastic cages containing hardwood bedding in groups of two of the same sex.

At 1.5 months and 18 months of age, the rats were tested in the spatial Morris water escape task and at 22 months they were processed for morphological analysis of presynaptic bouton numbers.

\section{Behavioural analysis}

Cognitive performance was assessed using the spatial Morris water escape task. The rats were first trained in this task when they were 1.5 months old. These finding were reported in a previous paper [29]. At 18 months of age, the rats were re-trained in the same task under identicall conditions (same room, same spatial cues). We used a modification of the protocol given by Prickaerts et al. [39]. A black water tank (diameter $1.22 \mathrm{~m}$ ) was divided into four quadrants, which were designated north, west, south and east (the last containing a hidden platform). "The rats ( $\mathrm{n}=7$ per group) were started facing the wall of the tank from different, 
randomly chosen, start positions, and trained to find the hidden platrorm (diameter $11 \mathrm{~cm}$ ) at a fixed invisible position ( $1 \mathrm{~cm}$ below the surface of the water) in the water tank. The water temperature was approximately $22^{\circ} \mathrm{C}$. A trial lasted until a rat had found the platform or until $60 \mathrm{~s}$ had elapsed. If the rat did not reach the platform within $60 \mathrm{~s}$, it was placed on the platform for $3 \mathrm{~s}$ and then removed from the water tank. The rats were trained during 2 days, with 2 trials a day. The time between subsequent trials was approximately $60 \mathrm{~min}$. Spatial cues were provided by including posters, a computer, a door, and by the presence of the experimenter. A radio was playing softly, providing a background noise during the testing period.

A probe trial was given 1 day before the rats were re-trained at 18 months of age in the spatial Morris water escape task. In this probe trial, the platform was removed and the rats had to swim for $60 \mathrm{~s}$. A second probe trial was given after the last trial on day two (at the end of the training period). All rats were started, facing the wall of the tank, opposite to the position of the removed platform. A video tracking system (Ethovision ${ }^{\mathrm{TM}}$ ) was used to record the movements of the rats automatically.

Three parameters of the spatial Morris water escape task were analysed: escape latency (time to reach the platform) ( $\mathrm{sec}$ ), total distance moved (cm) and swimming speed (cm/sec). Spatial learning ability was defined as the difference between trial $\mathbb{1}$ and 2 in escape latency and distance moved. Spatial short-time memory was defined as the difference between trial 2 on day 1 and trial 1 on day 2 in escape latency and total distance moved. Spatial long-term memory was defined as the difference between the last day of testing at 1.5 months and first day of testing at 18 months of age in total distance moved.

\section{Morphological analysis}

\section{Perfusion and tissue processing}

At 22 months, the rats ( $n=5$ for each group; randomly selected) were anaesthetized with sodium pentobarbital ( $60 \mathrm{mg} / \mathrm{kg}$; i.p.; Nembutal $)$ and perfused transcardially ( $50 \mathrm{mmHg}$ ), first with tyrode $(0.1 \mathrm{M})$, followed by the fixative containing $4 \%$ paraformaldehy de in $0.1 \mathrm{M}$ phosphate buffer (PB; pH 7.6; room temperature). Following perfusion, the brain was removed from the skull and postfixed for 24 hours at $4^{\circ} \mathrm{C}$ in the same fixative. Brain tissue was cryoprotected by immersion in $30 \%$ sucrose $/ 0.1 \mathrm{M}$ Tris-buffered saline (TBS) for 48 hours at $4{ }^{\circ} \mathrm{C}$. Afterwards, the brain was quickly frozen and stored at $-80^{\circ} \mathrm{C}$ until further processing. The brain was then cut into $30 \mu \mathrm{m}$ thick coronal section on a cryostat. Alternate sections were used either for irnmunohistochemical staining, or contrasted with $0.3 \%$ cresyl violet for supporting anatomical delineation of the neostriatum and the different layers of the cerebral cortex.

\section{Synaptoplyysin immunohistochemistry}

All immunohistochemical reactions were performed using free-floating sections. All sections were processed simultaneously to ensure that all were processed under identical conditions. TBS $(0.01 \mathrm{M})$ with $0.2 \%$ Triton $X-100$ (TBS-T) was used for washing and diluting antibodies. 
To minimize background staining, sections were pre-incubated in $5 \%$ normal goat serum (Sigma, Zwijndrecht, The Netherlands) at room temperature for $30 \mathrm{~min}$ and normal goat serum (2.5\%) was added to all solutions containing antibodies. A monoclonal antibody against synaptophysin (Boehringer-Mannheim, Germany) was used at a dilution of $1: 35$ overnight at $4^{\circ} \mathrm{C}$. After washing with TBS and TBS-T, the tissue was immersed in goat anti-mouse IgG (1:150; American Qualex, San Clemente, CA, USA) for $1 \mathrm{~h}$ at room temperature, followed by a monoclonal mouse anti-peroxidase antibody (1:50; Seralab, Crawley Down, Sussex, United Kingdom) with $5 \mathrm{mg} / \mathrm{ml}$ horseradish peroxidase (Sigma, type IV) added for $1 \mathrm{~h}$ at room temperature. After washing with TBS and TBS-T the sections were incubated in $0.3 \% 3,3 \%$ dlaminobenzidine tetrahydrochloride (DAB; Sigma) containing $0.03 \% \mathrm{H} 2 \mathrm{O} 2$ in TBS-T for 15 min at room temperature. All sections were washed with TBS and mounted on gelatine-coated glass slides. After air-drying, the sections were dehydrated in ascending concentrations of alcohol, cleared with microclair and coverslipped with Permount (Fisher, Montreal, Canada). The synaptophysin-immunoreactivity was restricted to small punctae with a morphological appearance that was not apparently different between animals or groups.

\section{Delineation of investigated brain regions}

Three regions of the brain were analysed in the present study: frontal and parietal cortex and neostriatum. Since the boundaries of these regions are difficult to determine, defined areas according to Paxinos and Watson (1986) were chosen for analysing the volume and synaptic density (see Figure 1). The frontal cortex was defined as the area between interaural 11.20 $\mathrm{mm}$ (anterior boundary) and $7.20 \mathrm{~mm}$ (posterior boundary). An arbitrary medial boundary consisted of a line drawn from the dorsal tip of left-brain to top of the corpus callossum. The lateral boundary consisted of a line from the ventral tip of the lateral ventricle to the top of the cortex in a specific angle of $45^{\circ}$. The parietal cortex was defined as the area adjacent to the frontal cortex, using the same anterior and posterior boundaries. A ventral boundary was set drawing a horizontal line from the area where the corpus callossum shows a distinct decrease in thickness. A specified region of the neostriatum was considered, which could be defined with unambiguous anatomical boundaries. The level at which the corpus callossum first crosses the midline (10.6 $\mathrm{mm}$ anterior of the interaural line) was used as anterior boundary and where the fornix joins the diencephallon $(8.2 \mathrm{~mm}$ anterior of the interaural position) was used as posterior boundary. The corpus callossum defines the dorsal and lateral boundaries, the lateral ventricle defines the medial edge, and arbitrary ventral boundary consisted of a line drawn from the ventral tip of the lateral ventricle to the rhinal fissure including a part of the nucleus accumbens (as described by [24]). The globus pallidus was excluded from the defined region in sections in which the imaginary line crossed its borders.

\section{Estimates of the density of synaptophysin-immunoreactive presynaptic boutons}

The density of synaptophysin-immunoreactive presynaptic boutons (SIPB) was estimated as described by Wong et al. [47]. Briefly, an Olympus BH-2 microscope equipped with a 100 


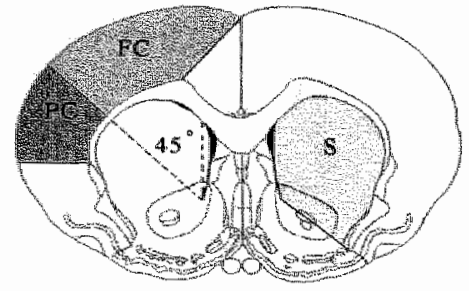

Interaural $11020 \mathrm{~mm}$

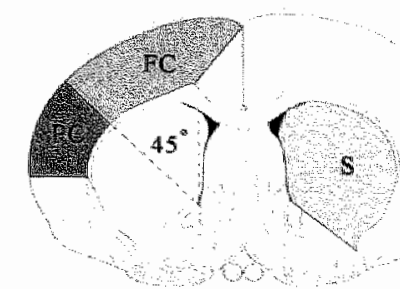

Interaural $9.48 \mathrm{~mm}$

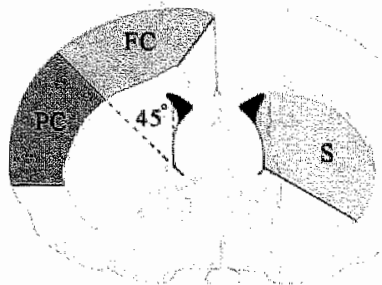

Interaural $8.74 \mathrm{~mm}$

Figure 1. Drawings of coronal sections through the rat brain (according to Paxinos and Watson [38]), showing the region of frontal cortex (FC) and parietal cortex (PC), and neostriatum (s) considered in the present study (shaded areas). The anterior limit was taken as the place in which the corpus callosum crosses the midline, and the caudal limit was the place in which the fornix joins the diencephalon (not shown). The medial boundary of the frontal cortex consisted of a line drawn of the dorsal tip of left brain to top of corpus callosum. The lateral boundary consisted of a line drawn from the ventral tip of the lateral ventricle to the top of the cortex in a specific angle, $45^{\circ} \mathrm{C}$. The parietal cortex was defined as the area next to the frontal cortex, using the same anterior and posterior boundaries. A ventral boundary was set drawing a horizontal line from the area where the corpus callosum shows a distinct decrease in thickness. The corpus callosum also defines the dorsal and lateral boundaries of the neostriatum. The lateral ventricle defines the medial edge, and an arbitrary ventral boundary consisted of a line drawn from the ventral tip of the lateral ventricle to the rhinal fissure, including part of the nucleus accumbens (as described by ingham et al. [24]). The globus pallidus was excluded from the defined region in sections in which the imaginary line crossed its borders.

oil immersion planachromatic objective and $x 10$ projection lens was used. The microscope was equipped with a CCD video (DAGE, MTC, CCD72, Michigan City, USA). Immunoreactive presynaptic boutons were detected by an image analysis system, slightly modified for detection of greyscale punctae (MCID M4; Imaging Research Inc., St. Catharines, Ontario, Canada). All measurements were performed on a single focal plane. Shading error correction was performed before measurements to correct for irregularities in illumination of the microscopic field. All images were saved on computer files and background levels equalized using the M3D software of the image analysis system. Measurements were performed on the equalized images. The detection thresholds were tested; corresponding values were stored in the computer program and kept at the same levels for all samples. Measurements were performed on 5 systematically and randomly sampled areas of $5,000 \mu \mathrm{m}^{2}$ in 6 systematically and randomly sampled sections of each investigated brain area. From these data, the SIPB density per unit 
area $\left(100 \mu \mathrm{m}^{2}\right)$ was calculated in each area and referred to as the presynaptic bouton density (DPB). The data of all layers of the cortex were pooled and referred to as presynaptic boutons global density (GDPB).

\section{Volume estimates}

Estimates of defined volumes (Vref) of the frontal and parietal cortex and neostriatum were carried out by means of the C.A.S.T. Grid software (Olympus, Albertslund, Denmark), using the point-counting method and the Cavalieri's principle [19]. By means of systematic and random sampling, a set of every sixth section of the entire brain regions was collected (immunohistochemically processed sections). The surface areas of the frontal and parietal cortex and neostriatum were measured on the left side; 8-10 sections per brain area were sampled. Volumes were measured at a final magnification of $x 3,600$ at approximately $900-$ 1,300 points systematically and randomly spaced throughout the frontal cortex, parietal cortex and neostriatum; point distance was $200 \mu \mathrm{m}$ by $200 \mu \mathrm{m}$. Volume estimates were calculated by multiplying the average surface area with the section thickness (post-processing thickness) and the number of investigated sections [19]. Post-processing thickness was measured to be able to compensate for shrinkage due to histological processing after dissection of the brains. Section thickness was measured using a microcator, which was attached to the microscope (see [45]). This microcator measured the distance between top (defined by the position where first cellular components came into focus) and bottom (defined by the position where the last cellular components were in focus) surfaces of the sections.

\section{Statistical analysis}

Results are given as mean \pm SEM. For the spatial Morris water escape task, data were analysed using ANOVA and Student's t-test. The differences in volume estimates, DPB and GDPB between SPA rats and CVD rats were tested using Student's t-test. The level of significance was set as $P<0.05$.

\section{Results}

\section{Behavioural analysis}

The data of the cognitive performance of the rats at 1.5 months have been published elsewhere by Loidl et al. [29]. Here data of the rats at 18 months are presented and compared with clata of 1.5 months of age. Figure 2 and 3 show the measurements of the acquisition of the spatial Morris water escape task and the performance in the probe trial. The time and total distance moved to reach the platform provide a measure of the ability to learn the task. Both CVD and SPA had acquired the task, as shown by the decrease in time (Figure $2 \mathrm{~A}$ ) and distance moved (Figure $2 B$ ) to reach the platform at the second trial on day one compared with the first trial 
A.

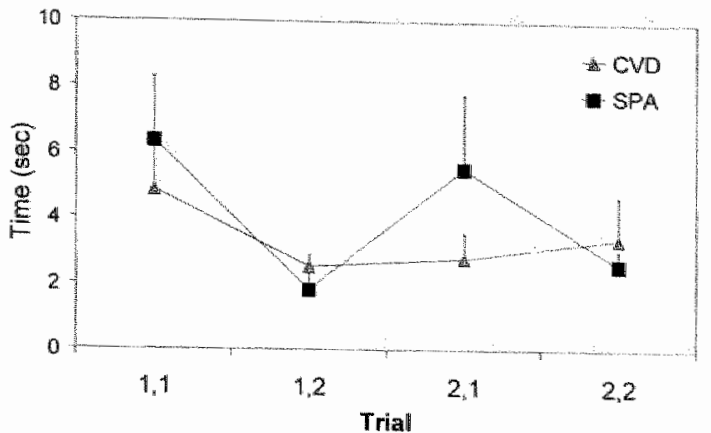

B.

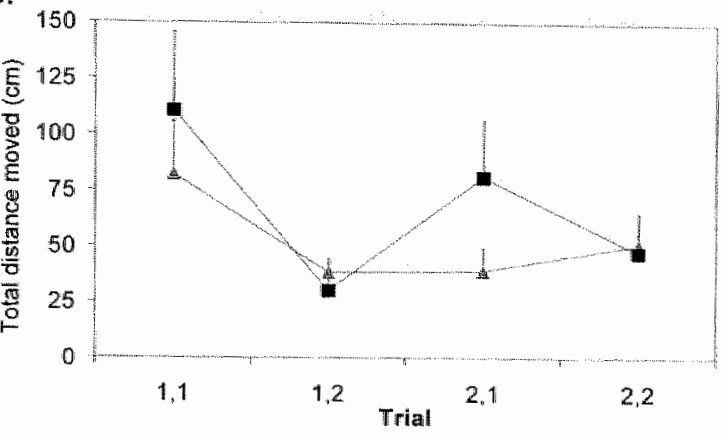

c.

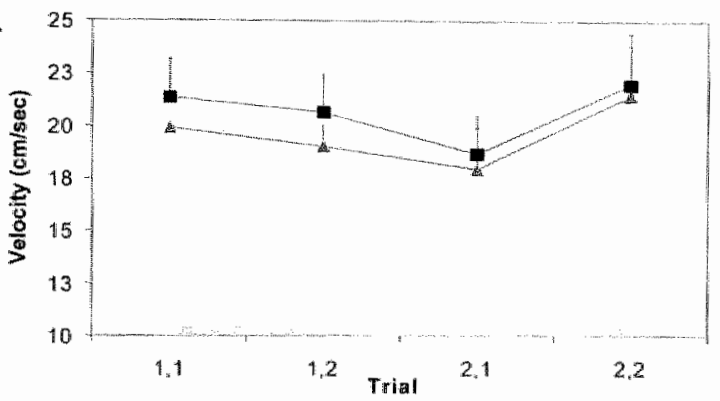

Figure 2. Results from spatial Morris water maze task of CVD rats (closed circles; $n=7$ ) and SPA rats (closed squares; $n=7$ ) at 18 months of age. Each rat was tested twice on one day (trial 1 and 2) and twice on the following day (trial 3 and 4). Values are presented as mean \pm SEM. (A) Time (seconds) needed to find the platform. Both CVD and SPA rats were able to find the platform and performed better on the second trial of the first day. The SPA rats were not able to remember the place of the platform on the second day of the experiment (increase in time to reach platform; trial 3 versus 2), in contrast to the CVD rats. The CVD rats did not perform better on the second trial (trial 4) of the second day, in contrast to the SPA rats. (B) Total distance moved $(\mathrm{cm})$ to find the hidden platform. Both CVD and SPA rats performed better on the second trial of the first day, as shown by a decrease in total distance moved (trial 2 versus trial 1). The SPA rats were not able to remember the position of the platform on the second day, as shown by increase of total distance moved (trial 3 versus trial 2). The CVD did not perform better on the second trial (trial 4) of the second day, in contrast to the SPA rats. (C) Mean swimming speed $(\mathrm{cm} / \mathrm{sec})$ during the tests. No differences were seen in mean velocity between CVD and SPA rats. 
on day one. Repeated measures ANOVA (trial, group) revealed a trial effect $(F(3,33)=3.14$; $P=0.04)$, but no effect of group, indicating that both CVD and SPA were equally efficient in re-learning the task. A group effect was seen comparing total distance moved of day 1 (trial 1 and 2) with day 2 (trial 3 and 4), indicating that asphyctic rats had more problems remembering the task than CVD. However, this difference was not statistically significant $(F(3,33)=2,48$; $P=0.08$ ). No differences were found in mean velocity (swim speed; Figure $2 \mathrm{C}$ ) between the different trials or between the CVD and SPA. In the probe trial that was done before the retraining period at 18 months, no quadrant preference was seen in the rats (both CVD and SPA). In the probe trial, which was done after the last trial at 18 months, all rats showed a quadrant preference $(P=0.03$; Figure 3$)$. The time spent in the training quadrant was the same for the CVD and SPA rats.

Multiple comparison between age groups demonstrated that the distance moved to reach the platform of 18 month-old rats at first day of the experiment was higher than that of 1.5 monthold rats at last day of experiment $(F(1,26)=3,32 ; \mathrm{P}=0.04)$. The data clearly show that spatial Morris water escape performance of all rats decreases from 1.5 month to 18 months (Fig. 4a). In addition, the increase in distance moved to reach the platform between 1.5 months and 18 months was greater in the SPA group than in the CVD group $(F(3,24)=3.26 ; \mathrm{P}=0.045$; Figure 4b).

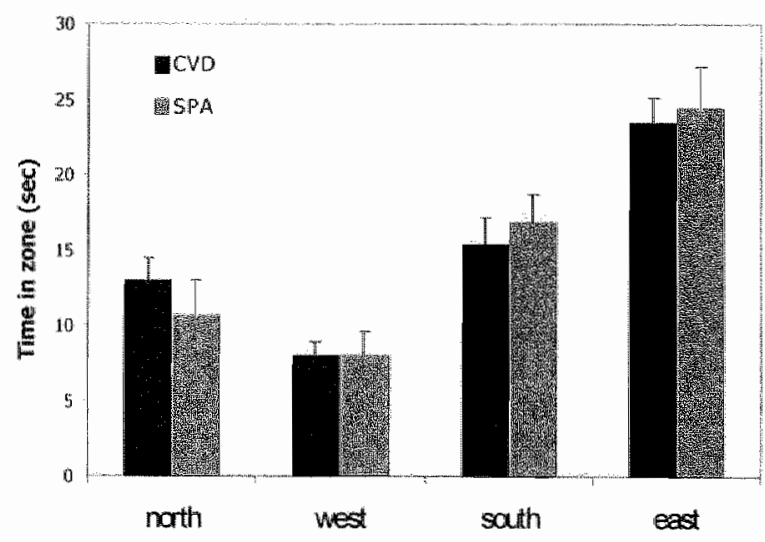

Figure 3. Results obtained from the probe trial of CVD rats (grey bars; $n=7$ ) and SPA rats (black bars; $n=7$ ) at 18 months of age. Each rat had to swim for $60 \mathrm{~s}$, and the times spent in the four quadrants of the water tank were recorded. The quadrants were organized with respect to the training quadrant, i.e. north, west, south and east (the last containing the hidden platform). Values are given as means \pm SEM. Both CVD and SPA rats had a preference for quadrant 4 (east). No differences were found between the groups. 


\section{Morphological analysis}

\section{Density of synaptophysin-immunoreactive presynaptic boutons}

Figure 5 shows synaptophysin-immunostaining in parietal cortex and examples of edited images, showing the detection of SIPB by the image analysis system. Figure 6 displays the results obtained for DPB in the different layers of the frontal and parietal cortex of CVD and SPA rats. For the frontal cortex, there were no significant differences between CVD and SPA animals. By contrast, for the parietal cortex a significantly greater DPB was found for SPA animals as for CVD animals in layer 4 $(P=0.012)$, layer $5(P=0.048)$ and layer $6(P=0.030)$. Figure 7 shows the results obtained for GDPB in frontal cortex, parietal cortex and neostriatum of CVD and SPA rats. For frontal cortex and neostriatum, no significant difference was found between CVD and SPA animals. By contrast, SPA animals showed a significantly greater GDPB in parietal cortex than $\mathrm{CVD}$ animals $(+10.4 \% ; P=0.04)$. This difference was more apparent in the deeper cortical layers than in superficial layer of parietal cortex $(12.8 \%$ in layer $4-6$ and $8.1 \%$ in layer $1-3$ ).
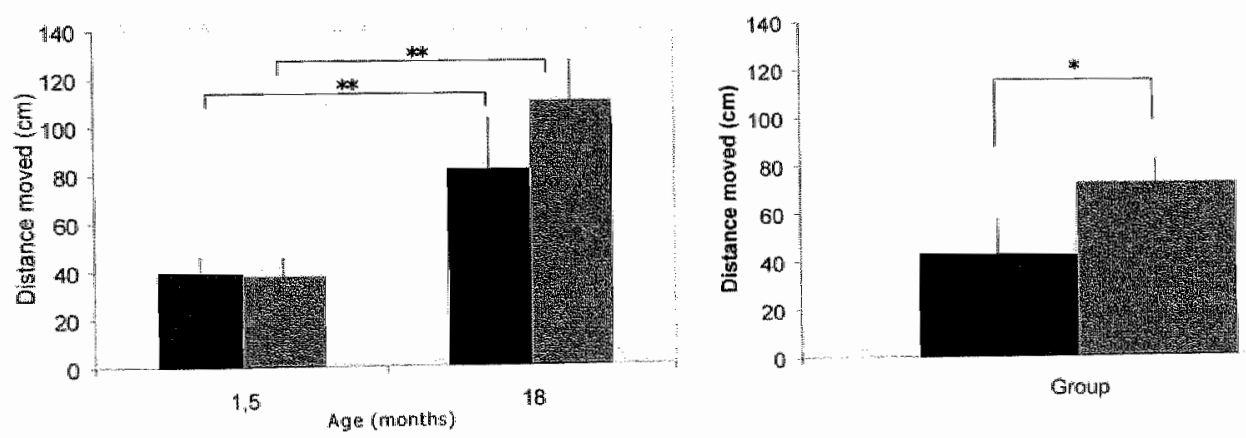

Figure 4. Results obtained from spatial Morris water escape task of CVD rats (gray bars; $n$ $=7$ ) and SPA rats (black bars; $n=7$ ) at either 1.5 or 18 months of age. Each rat was tested wice on one day and twice on the following day. Data shown for 1.5 months old rats represent means $( \pm$ SEM) of the two trials on the last day of the experiment, while data shown for 18 months old rats represent means $( \pm S E M)$ of the two trials on the first day of the experiments. A. Distances moved of 1.5 months and 18 months old rats. Both CVD and SPA rats performed worse at 18 months of age, as shown by an increase in total distance moved. B, Differences between the distance moved at 1.5 months of age and the distance moved at 18 months of age of CVD and SPA rats. The differences between distance moved at 1.5 months and 18 months of age were more pronounced in SPA rats than in CVD rats. $P<0.05$; **P<0.01. 


\section{Volume estimates}

Figure 8 shows the results of the volume estimates of the frontal cortex, parietal cortex and neostriatum. For none of the investigated brain areas, significant differences were found between CVD rats and SPA rats. However there was a trend towards an increased volume of the neostriatum following perinatal asphyxia $(+9,6 \% ; P=0.10)$.

Post-processing thickness of the sections was $18 \mu \mathrm{m} \pm 1 \mu \mathrm{m}$ for all rats (no difference between groups).

\section{Total numbers of synaptophysin-immunoreactive presynaptic boutons}

From the data presented here it is not possible to calculate total number of presynaptic boutons, since size, shape and geometric orientation of the presynaptic boutons are unknown. However, no apparent morphological differences (Nissl staining) were found between CVD and SPA rats. Considering the increased DPB in layer 4 to 6 of the parietal cortex (as well as the increased GDPB in this brain area) and the unchanged volume of this brain area in SPA animals, it was reasonable to conclude that there was a tendency towards an increase of total number of presynaptic boutons in the parietal cortex in the adult asphyctic rats. Furthermore, considering the unchanged GDPB in the neostriatum and the increased volume of this brain area in SPA animals, it was reasonable to conclude that there was a tendency towards an increase of total number of presynaptic boutons in the neostriatum in the adult asphyctic rats.
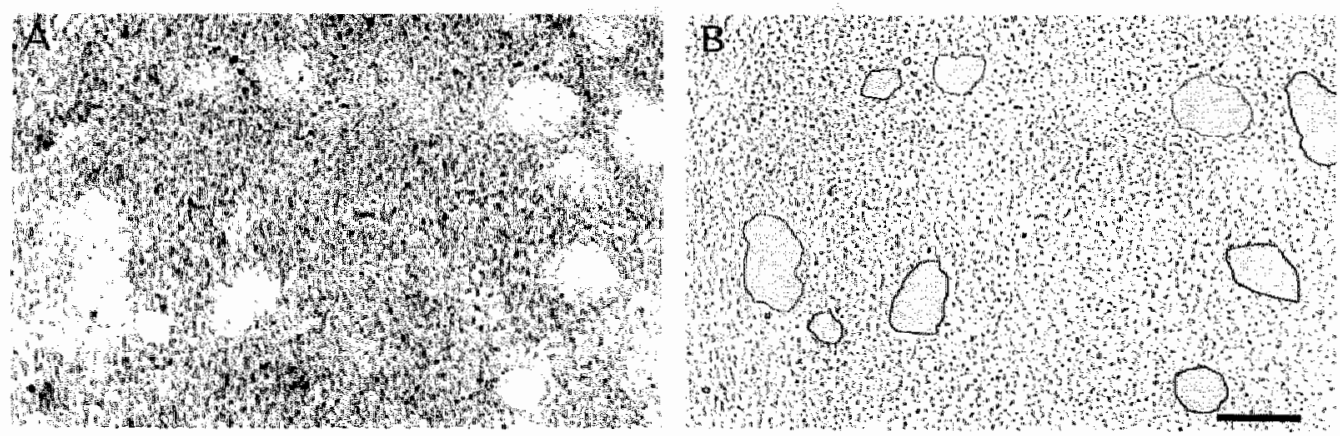

Figure 5. Example of an image of synaptophysin-stained area in parietal cortex as obtained with the image analysis system. (A) Unedited gray level image. (B) Edited image showing the detection of the immunoreactive dots by the image analysis system. Blood vessels, neuronal cell bodies and the area not in focus were excluded from the analyses (grey areas). Scale bar $=10 \mu \mathrm{m}$. 

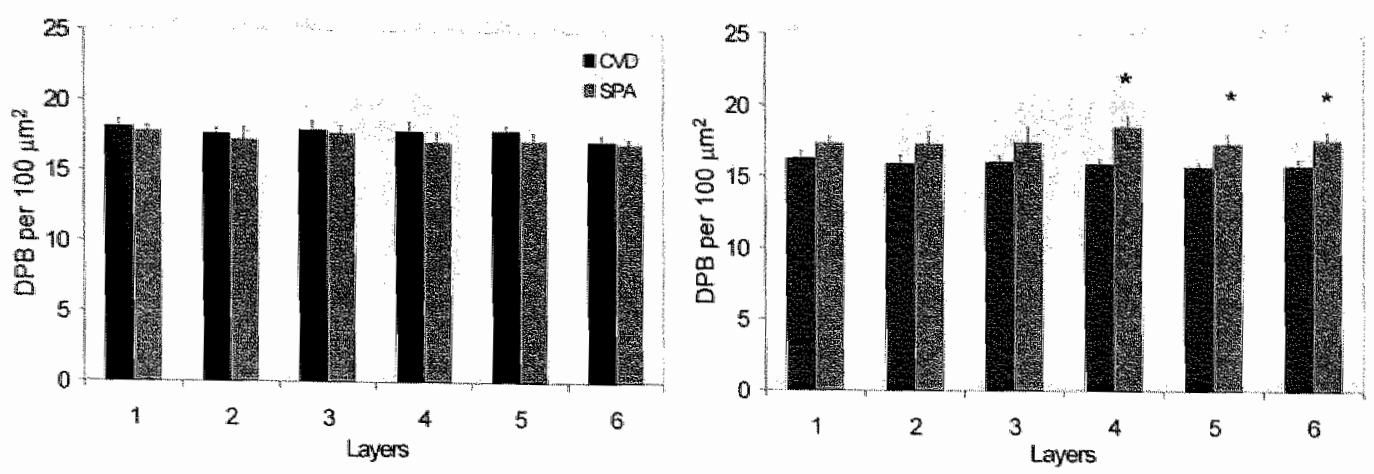

Figure 6. Synaptophysin-immunoreactive presynaptic boutons per $100 \mu \mathrm{m}^{2}$ (DPB) in different layers of the cortex of CVD rats (grey bars; $n=5$ ) and SPA rats (black bars; $n=5$ ) at 18 months of age. Data are expressed as mean \pm SEM. (A) DPB in different layers of frontal cortex. There were no significant differences between CVD and SPA rats. (B) DPB in different layers of parietal cortex. In layers 4,5 and 6, a significant increase in DPB was found in SPA rats compared to CVD rats. ${ }^{*} P<0.05$.

\section{Discussion}

\section{Age-related reduction in cognitive performance of asphyctic rats}

In the present study, we show that perinatal asphyxia leads to exaggerated age-related longterm memory impairment. However, perinatal asphyxia did not lead to deficits in learning ability or short-term memory. Spatial discrimination learning involves place learning (i.e. learning a position in space) as well as non-spatial components such as procedural learning (i.e. learning to search for an escape platform), sensorimotor processes and possible motivational processes necessary for executing the task $[35,39]$.

The spatial Morris water escape task has proven to be an exceptionally useful tool for assessing the spatial and non-spatial memory abilities of rats $[5,39]$. During the acquisition of the spatial Morris water escape task, the rats learned to locate the platform that was in a fixed spatial location relative to distal room cues [35]. The probe trial can reveal whether the rats have actually learned the position of the platform. Place learning was considered by placement of the platform in one of the four positions of the maze, while the rat was trained two trails/day. This learning procedure is considered analogous to rapid acquisition memory processes, e.g., working memory, although still being a cognitive mapping-learning task [5]. This assumes that the spatial Morris water escape task as performed in these studies is mainly a test for cortical function. However, swim speed (mean velocity) of the rats in this task can be related to motor and motivational behaviour, which is not only related to the motor cortex but also to the neostriatal area $[1,26]$. 
A

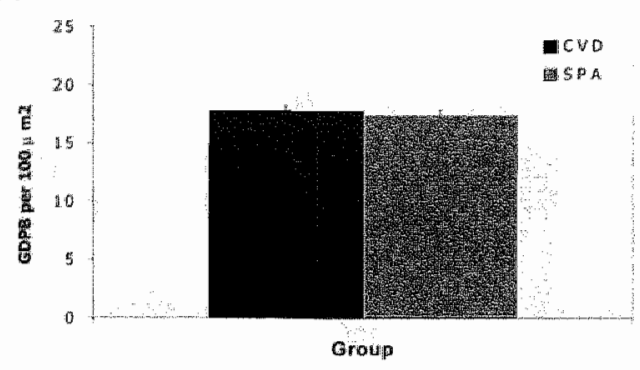

B

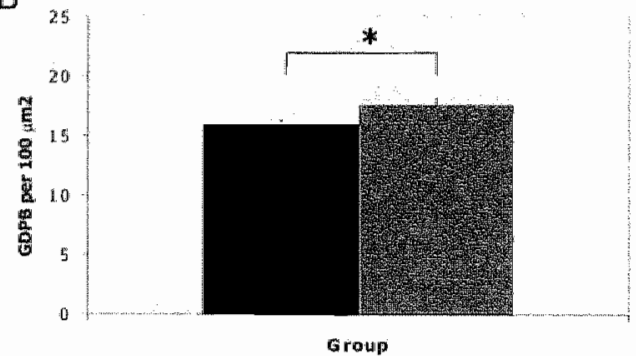

C

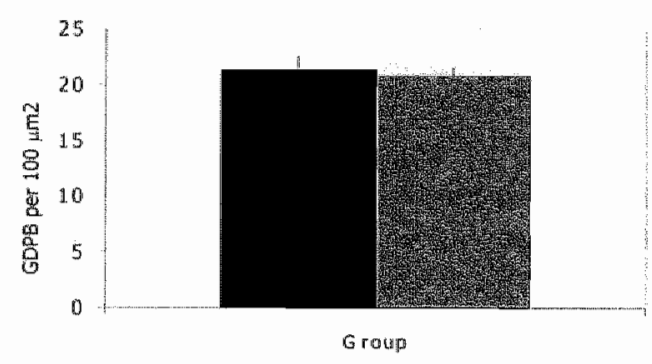

Figure 7. Global density of synaptophysinimmunoreactive presynaptic boutons (GDPB; calculated as number of presynaptic boutons per $100 \mu \mathrm{m}^{2}$ ) in frontal cortex, parietal cortex and neostriatum of CVD rats (grey bars; $n=5$ ) and SPA rats (black bars; $n$ $=5$ ) at 18 months of age. (A) GDPB in frontal cortex. No significant differences were found between CVD and SPA rats. (B) GDPB in parietal cortex. An increase in GDPB was found in SPA rats, compared to SPA rats. (C) GDPB in striatum. No significant differences were found between CVD and SPA rats.

* $P<0.05$.
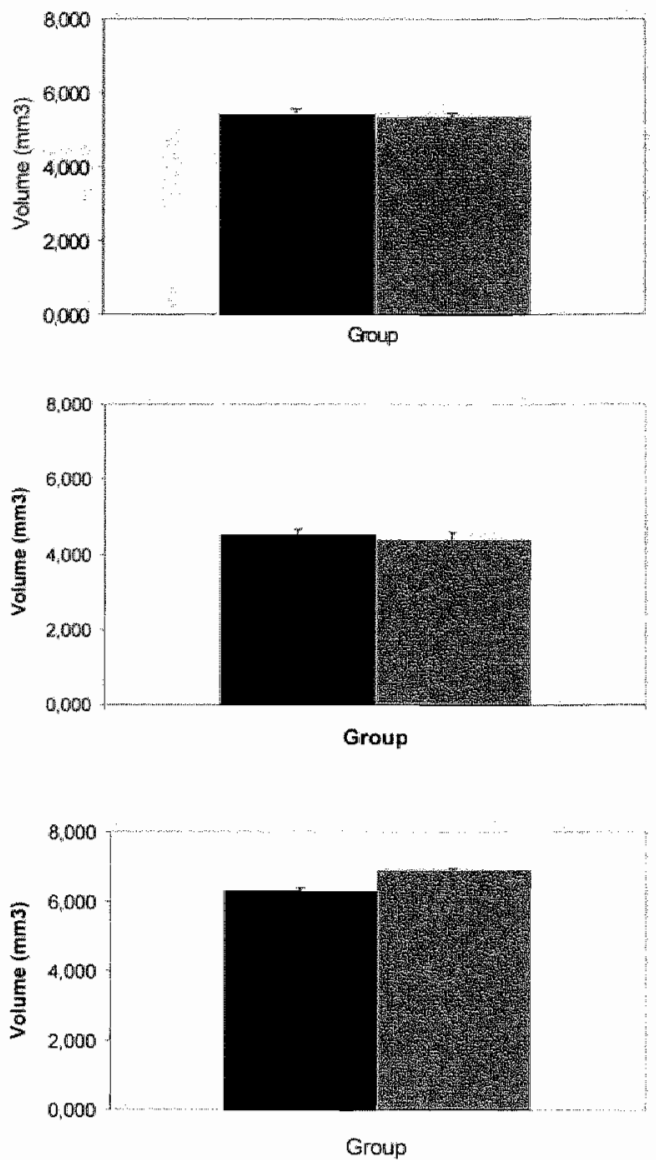

Figure 8. Volume estimates of frontal cortex (A), parietal cortex $(B)$ and neostriatum (C) of CVD rats (grey bars; $n=5$ ) and SPA rats (black bars; $n=5$ ) at 18 months of age. There were no significant differences between CVD and SPA animals. 
The findings presented here suggest that the performance of SPA rats in the spatial task during the acquisition and in the probe trial (both trials) did not differ from CVD rats at 18 months of age. This indicates that place learning and visual and sensorimotor processes were not affected in the SPA rats. The finding that both CVD and SPA rats improved their performance on the first day (trial 1 vs. trial 2) indicated that asphyxia did not affect the spatial learning ability in rats. Swim speed of aged SPA rats in the spatial Morris water escape task did not differ from swim speed of aged CVD rats, showing that motor processes in both groups were not disturbed. When comparing the individual performance of the rats from the first acquisition (1.5 months, last day) with the second acquisition ( 18 months, first day), it was found that the rats performed worse at 18 months of age.

Cognitive impairment as a consequence of aging has been related to a reduction in synapses in parietal cortex [47]. Furthermore, aged "impaired" rats also show a significant reduction of cholinergic neurons (ChAT) in striatum and frontal and sensomotor cortices, which have proven to be related to place-learning deficits [12-14,21]. However, it might be noted that aged 'impaired' rats also show alterations in other neurotransmitter systems, such as the dopaminergic and glutamatergic system [15,49]. The observed difference in distance moved between young and aged rats was more pronounced in SPA rats than in CVD rats, suggesting a more pronounced impairment in cognitive performance in aged SPA rats.

Previous studies have shown that perinatal asphyxia can lead to neuron loss in cerebral cortex and striatum $[27,41]$. Furthermore, perinatal asphyxia can lead to a decrease in spontaneous locomotor activity in the open field task [29] and damage to dopaminergic and cholinergic neurotransmitter systems $[11,30]$. Taken together, this would indicate that damage to the dopaminergic system and/or cholinergic system might lead to a reduction in cognitive performance and a disturbance of the motor system.

Furthermore, this would indicate that the reduction in cognitive performance might be due to a disturbance of the motor system. Finally, these studies support the idea that perinatal asphyxia can result in cortical changes besides the already reported alterations of striatal and hippocampal area $[10,27]$.

\section{Morphological analysis}

\section{Validity of methods and sources of error}

There is a controversy in the literature about the validity and usefulness of the several methods used to quantify presynaptic boutons. Most problems arise during the delineation of the region of interest, the sampling scheme and choice of counting method $(\operatorname{cf}[8,44])$. In addition, all counting methods have their advantages and disadvantage. Quantification of synapses at the ultrastructural level is the onlly available way to visualize individual synaptic contacts, but is extremely time-consuming and complex. Quantification of presynaptic boutons using synaptophysin-immunostaining is simple, but assumes that the size, shape and geometic orientation of the synapses are not changed in an experimental group versus a control group. Furthermore, alterations of presynaptic bouton density have to be interpreted 
in a careful way, because it can be the consequence of a change in total number of presynaptic boutons $(\mathrm{N}$ ), change in volume of the structure (Vref) or changes in both (cf [44]). Stereology can provide accurate measurements of total number of presynaptic boutons without assumptions stemming from tissue processing of momphological changes. However this method is dependent on magnification and can only be termed 'unbiased' if the resolution of the immunostained boutons is uniform throughout the section [6].

All these methods were considered for the present study. We combined light-microscopic image analysis of synaptophysin-stained presynaptic boutons in the striatum and the cerebral cortex with volume estimates of the corresponding brain areas. This method was used since ultrastructural analysis was extremely difficult and stereological methods (e.g. optical disector) were unfit for this analysis since the resolution of the immunostained boutons was not uniform throughout the section. Density estimates of presynaptic boutons have given accurate detection of relative changes of this variable in the cerebral cortex of human neuropathological material $[2,32]$ and in transgenic animals models [16,31]. Furthermore, volume estimates using the point counting method and the Cavalieri's principle have become the state-of-the-art in modern stereology (cf [19]).

The accuracy of the measurements can be easily affected by variations in the intensities and qualities of the immunostaining and the illumination settings of the microscope. These problems can be avoided by measurement of the number of presynaptic boutons per unit area using image analysis [47]. In addition, the accuracy of the measurements can be affected by the delineation of the region of interest and the sampling scheme of investigated areas and sections. To obtain accurate measurements of presynaptic bouton densities per unit area, we systematically and randomly sampled both areas and sections throughout the investigated brain areas. Definitions of borders of these brain regions were made according to Paxinos and Watson [38]. Threshold settings were fixed and same settings were used for all investigations. Shading error corrections were performed before measurements to correct for irregularities in illumination in the microscopic field. We also performed a further correction using the M3D module of the software to allow for compensation of indifferences in background levels of the immunostained areas. During actual measurements from the saved images, detection levels were kept the same during all quantifications, so that valid and unbiased data could be gathered. Furthemore, the volume estimates were done using a thigh magnification objective $(\times 100)$ to avoid overprojection (i.e. overcounting of synapes) during the investigation of the relatively thick sections. Therefore, correction of the volume estimates with respect to overprojection was not necessary in this study. In addition, high magnification objectives can provide accurate estimates of the number of presynaptic boutons with diameters in the one to two micron range [28]. The small size of presynaptic boutons (in contrast to neuronal cell bodies) simplified stereological protocols as their small size minimizes the potential overlapping of features between adjacent sections or focal planes.

Our data emphasize the repeated waming in the literature to use dlensity measurements always in conjunction with volume measurements [20]. A combined approach using light-microscopic analysis of presynaptic boutons density and volume estimates can provide clear evidence of the change in presynaptic bouton number in a defined area. 


\section{Perinatal asphyxia increases presynaptic bouton numbers in striatum and parietal cortex}

In this study, we show that asphyctic injury during the development of the brain can have a long-lasting effect on the synaptic organization of the brain, especially in the striatum and the parietal cortex. Alterations in presynaptic bouton density of the parietal cortex have been previously reported in relation to aging. For example, Wong er al. [47] have shown in Brown Norway $x$ Fischer $344 \mathrm{~F} 1$ hybrid rats that aging can be related to a decrease in presynaptic bouton density in the parietal cortex. A loss of synaptic input was also reported in the frontal cortex in humans comparing young with older individuals [32]. However, an increase in presynaptic bouton density has only been reported as a direct result of damage to the brain tissue. An increase in presynaptic bouton density in the cortex was reported as a consequence of cerebral ischemia [40] or as a consequence of decortication [17]. The observed increase in presynaptic bouton numbers in parietal cortex and striatum in asphyctic rats supports the hypothesis that brain damage during brain development can not only lead to synaptic loss, but also to the formation of new synaptic connections. An increase in presynaptic bouton numbers would allow for more neural communication and could compensate for a loss of neurons. However, the increase in presynaptic bouton numbers shown here did not compensate for long-term memory impairment.

In conclusion, perinatal asphyxia leads to an augmented age-related cognitive impairment and an increase in total number of presynaptic boutons in the parietal cortex and striatum. This synaptic plasticity induced by perinatal asphyxia was region-specific. The mechanism related to this type of synaptic plasticity is still unknown. It is expected that future studies and developments in the field should shed. more light on the mechanisms underlying the compensatory role of synaptogenesis in the restoration of the damaged CNS during development.

\section{Acknowledgements}

The authors gratefully acknowledge the expert technical assistance provided by Mrs Hellen Steinbusch and Sylvain Coté. We are grateful to Dr. Alfredo Ribeiro-Da-Silva for his helpful discussions. Furthermore, we would like to thank Michèl Aquarius for assistance in the morphological analysis. 


\section{References}

[1] Alexander, G.E. and Crutcher, M.D. 1990. Functional architecture of basal ganglia circuits: neural substrates of parallel processing. Trends Neurosci. 13: 266-71.

[2] Alford, M.F, Masliah, E., Hansen, L.A. and Terry, R.D. 1994. A simple dot-immunobinding assay for quantification of synaptophysin-like immunoreactivity in human brain. J Histochem Cytochem. 42: 283-7.

[3] Black, J.E. Isaacs, K.R., Anderson, B.J., Alcantara, A.A. and Greenough, W.T. 1990. Learning causes synaptogenesis, whereas motor activity causes angiogenesis, in cerebellar cortex of adult rats. Proc Natl Acad Sci U S A. 87: 5568-72.

[4] Boksa, P., Krishnamurthy, A. and Brooks, W. 1995. Effects of a period of asphyxia during birth on spatial learning in the rat. Pediatr Res. 37: 489-96.

[5] Brandeis, R., Brandys, Y. and Yehuda, S. 1989. The use of the Morris Water Maze in the study of memory and learning. Int $J$ Neurosci. 48: 29-69.

[6] Calhoun, M.E., Jucker, M., Martin, L.J., Thinakaran, G., Price, D.L. and Mouton, P.R. 1996. Comparative evaluation of synaptophysin-based methods for quantification of synapses. $J$ Neurocytol. 25: 821-8.

[7] Calhoun, M.E., Kurth, D., Phinney, A.L., Long, J.M., Hengemihle, J., Mouton, P.R., Ingram, D.K. and Jucker, M. 1998. Hippocampal neuron and synaptophysin-positive bouton number in aging C57BL/6 mice. Neurobliol Aging. 19:599-606.

[8] Coggeshall, A.E. and Lekan, H.A. 1996. Methods for determining numbers of cells and synapses: a case for more uniform standards of review. J Comp Neurol. 364: 6-15.

[9] Dalman, C., Allebeck, P., Cullberg, J., Grunewald, C. and Koster, M. 1999. Obstetric complications and the risk of schizophrenia: a longitudinal study of a national birth cohort. Arch Gen Psychiatry. 56: 234-40.

[10] Dell'Anna, E., Chen, Y., Engidawork, E., Andersson, K., Lubec, G., Luthman, J. and HerreraMarschitz, M. 1997. Delayed neuronal death following perinatal asphyxia in rat. Exp Brain Res. 115: 105-15.

[11] El-Khodor, B.F. and Boksa, P. 1998. Birth insult increases amphetamine-induced behavioral responses in the adult rat. Neuroscience. 87: 893-904.

[12] Fischer, W., Chen, K.S., Gage, F.H. and Bjorklund, A. 1992. Progressive decline in spatial learning and integrity of forebrain cholinergic neurons in rats during aging. Neurobiol Aging. 13: 9-23.

[13] Fischer, W, Nilsson, O.G. and Bjorklund, A. 1991. In vivo acetylcholine release as measured by microdialysis is unaltered in the hippocampus of cognitively impaired aged rats with degenerative changes in the basial forebrain. Brain Res. 556: 44-52.

[14] Fischer, W.,Wictorin, K., Bjorklund, A., Williams, L.R., Varon, S. and Gage, F.H. 1987. Amelioration of cholinergic neuron atrophy and spatial memory impairment in aged rats by nerve growth factor. Nature. 329: 65-8.

[15] Gallagher, M., Burwell, R.D., Kodsi, M.H., McKinney, M., Southerland, S. Vella-Rountree, $L$. and Lewis,$M . H .1990$. Markers for biogenic amines in the aged rat brain: relationship to decline in spatial learning ability. Neurobiol Aging. 11:507-14.

[16] Games, D. Adams, D., Alessandrini, R., Barbour, R. Berthelette, P., Blackwell, C., Carr, T., Clemens, J., Donaldson, T., Gillespie, Fn and et al. 1995. Alzheimer-type neuropathology in transgenic mice overexpressing V717F beta-amyloid precursor protein. Nature. 373: 5237.

[17] Garofalo, L., Ribeiro-da-Silva, A. and Cuello, A.C. 1992. Nerve growth factor-induced synaptogenesis and hypertrophy of cortical cholinergic terminals. Proc Natl Acad Sci U S A. 89: $2639-43$. 
[18] Geinisman, Y., Disterhoft, J.F., Gundersen, H.J., McEchron, M.D., Persina, I.S., Power, J.M., van der Zee, E.A. and West, M.J. 2000. Remodeling of hippocampal synapses after hippocampus-dependient associative learning. J Comp Neurol. 417: 49-59.

[19] Gundersen, H.J. and Jensen, E.B. 1985. Stereological estimation of the volume-weighted mean volume of arbitrary particies observed on random sections. J Microsc. 138: 127-42.

[20] Heinsen, H., Rub, U., Bauer, M., Ulmar, G., Bethke, B., Schuler, M., Bocker, F., Eisenmenger, W., Gotz, M., Korr, H. and Schmitz, C. 1999. Nerve cell loss in the thalamic mediodorsal nucleus in Huntington's disease. Acta Neuropathol (Berli). 97: 613-22.

[21] Hellweg, R., Fischer, W., Hock, C., Gage, F.H., Bjorklund, A. and Thoenen, H. 1990. Nerve growth factor levels and choline acetyltransferase activity in the brain of aged rats with spatial memory impairments. Brain Res. 537: 123-30.

[22] Hoeger, H., Engelmann, M., Bernert, G. Seidl, R., Bubna-Littitz, H., Mosgoeller, W., Lubec, $B$. and $L u b e c, G$. 2000. Long term neurological and behavioral effects of graded perinatal asphyxia in the rat. Life Sci. 66: 947-62.

[23] Hultman, C.M., Sparen, P., Takei, N., Murray, R.M. and Cnattingius, S. 1999. Prenatal and perinatal risk factors for schizophrenia, affective psychosis, and reactive psychosis of early onset: case-control study. Bmj. 318: 421-6.

[24] Ingham, C.A., Hood, S.H., Taggart, P. and Arbuthnott, G.W. 1998. Plasticity of synapses in the rat neostriatum after unilateral lesion of the nigrostratal dopaminergic pathway. J Neurosci. 18: $4732-43$.

[25] Jones, P.B., Rantakallio, P., Hartikainen, A.L., Isohanni, M. and Sipila, P. 1998. Schizophrenia as a long-term outcome of pregnancy, delivery, and perinatal complications: a 28-year followup of the 1966 north Finland general population birth cohort. Am J Psychiatry. 155: 355-64.

[26] Kalaska, J.F. and Crammond, D.J. 1992. Cerebral cortical mechanisms of reaching movements. Science. 255: 1517-23.

[27] Kohlhauser, C., Kaehler, S. Mosgoeller, W., Singewald, N., Kouvelas, D. Prast, H., Hoeger, $H$. and Lubec, B. 1999. Histological changes and neurotransmitter levels three months following perinatall asphyxia in the rat. Life Sci. $64: 2109-24$.

[28] Kuroda, M. and Price, J.L. 1991. Ultrastructure and synaptic organization of axon terminals from brainstem structures to the mediodiorsal thalamic nucleus of the rat. $J$ Comp Neurol. 313: 539-52.

[29] Loidl, C.F., Gavilanes, A.W., Van Dijk, E.H., Vreuls, W., Blokland, A., Vles, J.S. Steinbusch, H.W. and Blanco, C.E. 2000. Effects of hypothermia and gender on survival and behavior after perinatal asphyxia in rats. Physiol Behav. 68: 263-9.

[30] Loidl, C.F., Herrera-Marschitz, M., Andersson, K., You, Z.B., Goiny, M., O'Connor, W.T., Silveira, R., Rawal, R., Bjelke, B., Chen, Y. and et al. 1994. Long-term effects of perinatal asphyxia on basal ganglia neurotransmitter systems studied with microdialysis in rat. Neurosci Lett. 175: 9-12.

[31] Masliah, E. Ilwai, A., Maliory, M., Ueda, K, and Saitoh, T. 1996. Altered presynaptic protein NACP is associated with plaque formation and neurodegeneration in Alzheimer's disease. Am J Patholl. 148: 201-10.

[32] Masiliah. E., Mallory, M., Hansen, L., DeTeresa, R. and Terry, R.D. 1993. Quantitative synaptic alterations in the human neocortex during normal aging. Neurology. 43: 192-7.

[33] McDonald, C. and Murray, R.M. 2000. Early and late environmental risik factors for schizophrenia. Brain Res Brain Res Rev. 31: 130-7.

[34] McNeil, T.F., Cantor-Graae, E. and Ismail, B. 2000. Obstetric complications and congenital malformation in schizophrenia. Brain Res Brain Res Rev. 31: 166-78.

[35] Morris, R. 1984. Developments of a water-maze procedure for studying spatial learning in the rat. $J$ Neurosci Methods. 11: 47-60. 
[36] Nicolle, M.M. Gallagher, M. and McKinney, M. 1999. No loss of synaptic proteins in the hippocampus of aged, behaviorally impaired rats. Neurobiol Aging. 20: 343-8.

[37] Palmer, C. and Vannucci, R.C. 1993. Potential new therapies for perinatal cereloral hypoxiaischemia. Clin Perinatol. 20:411-32.

[38] Paxinos, G., Watson, C. 1986. The rat brain in stereotaxic coordinates. Academic press, Sydney.

[39] Prickaerts, J., De Vente, J., Markerink-Van Ittersum, M. and Steinbusch, H.W. 1998. Behavioural, neurochemical and neuroanatomical effects of chronic postnatal $\mathrm{N}$-nitro-L. arginíne methyl ester treatment in neonatal and adult rats. Neuroscience. 87: 181-95.

[40] Stroemer, R.P., Kent, T.A. and Hulsebosch, C.E. 1992. Increase in synaptophysin immunoreactivity folllowing cortical infarction. Neurosci Lett. 147: 21-4.

[41] Vande Berg, W.D., Schmitz, C., Steinbusch, H.W. and Blanco, C.E. 2002. Perinatal asphyxia induced neuronal loss by apoptosis in the neonatal rat striatum: a combined TUNEL and stereological study. Exp Neurol. 174: 29-36.

[42] Verdoux, H., Geddes, J.R., Takei, $\mathbb{N}_{\text {. }}$ Lawrie, S.M., Bovet, P., Eagles, J.M., Heun, $\mathbb{R}$., McCreadie, R.G., McNeil T.F., O'Callaghan, E., Stober, G., Willinger, M.U., Wright, P. and Murray, R.M. 1997. Obstetric complications and age at onset in schizophrenia: an international collaborative meta-analysis of individual patient data. Am J Psychiatry. 154: 1220-7.

[43] Volpe, J.J. 1992. Brain injury in the premature infant-current concepts of pathogenesis and prevention. Biol Neonate. $62: 231-42$.

[44] West, M.d. 1999. Stereological methods for estimating the total number of neurons and synapses: issues of precision and bias. Trends Neurosci. 22: 51-61.

[45] West, M.J., Ostergaard, K., Andreassen, O.A. and Finsen, B. 1996. Estimation of the number of somatostatin neurons in the striatum: an in situ hybridization study using the optical fractionator method. J Comp Neurol. 370: 11-22.

[46] Wiedenmann, B. and Franke, W.W. 1985. Identification and localization of synaptophysin, an integrall membrane glycoprotein of $\mathrm{Mr} 38,000$ characteristic of presynaptic vesicles. Cell. 41: 1017-28.

[47] Wong, T.P., Campbell, P.M., Ribeiro-da-Silva, A. and Cuello, A.C. 1998. Synaptic numbers across cortical laminae and cognitive performance of the rat during ageing. Neuroscience. 84: 403-12.

[48] Younkin, D.P. 1992. Hypoxic-ischemic brain injury of the newborn-statement of the problem and overview. Brain Pathol. 2: 209-10.

[49] Zhang, W.Q. Mundy, W. R. Thai "L., Hudson, P.M. Gallagher, M., Tilson, H. A. and Hong, J.S. 1991. Decreased glutamate release correlates with elevated dynorphin content in the hippocampus of aged rats with spatial learning deficits. Hippocampus. 1: $391-7$. 


\section{Hypothermia prevents cytoplasmic stress gene response induced by perinatal asphyxia}

W.D.J. Van de Berg, W. Paschen, A. Scheepens, H.W.M. Steinbusch, C.E. Blanco 


\section{Abstract}

In the present study, we investigated the effect of post-asphyxia hypothermia on the stress gene response using semi-quantitative RT-PCR. Asphyxia was induced at term by immersing fetus-containing uterus horns of Wistar dams in a water bath at $37^{\circ} \mathrm{C}$ for $19 \mathrm{~min}$. Rat pups were placed at random in a paediatric incubator at $37^{\circ} \mathrm{C}$ (normothermia) or $21^{\circ} \mathrm{C}$ (hypothermia) for $45 \mathrm{~min}$, before placement with the surrogate mother. Rat pups were sacrificed at 6 and 24 hrs after the insult. During the hypothermic period, the oral temperature of the rat pups dropped to $24^{\circ} \mathrm{C}$ and was maintained or less then $34^{\circ} \mathrm{C}$ for a $90 \mathrm{~min}$ period. Our results demonstrate a strong induction of hsp $70 \mathrm{mRNA}$ within the striatum and cerebellum, but not in the cerebral cortex, during the first 24 hrs after perinatal asphyxia. Grp78 mRNA levels and xbp-1 processing was unchanged following perinatal asphyxia. Hypothermia prevented the injury induced hsp70 mRNA induction, whereas it did not affect grp78 mRNA levels or XBP-1 processing. Therefore, we conclude that hypothermia can prevent the induction of cytoplasmic stress following perinatal asphyxia without affecting endoplasmic reticulum function. 


\section{Introduction}

Infants who suffer perinatal asphyxia frequently develop cerebral injury that may result in lifelong behavioural impairment. Cellular events occurring during and after perinatal asphyxia, including acidosis, calcium influx, release of excitatory amino acids, mitochondrial dysfunction and free radical formation $[22,38]$, lead to major neuronal damage and eventually death within vulnerable brain regions, such as the basal ganglia, the hippocampus and the cerebellum $[21,24,31]$. At present, there are no treatment strategies that have proven to be both effective and safe within the clinic.

Mild post-asphyxia hypothermia has proven to be one of the most potent neuroprotective strategies in animal models of brain injury. Mild hypothermia during the first $5.5 \mathrm{~h}$ after the insult, for example, has proven to be effective in reducing energy failure, neurochemical damage and conveys long-term neuroprotection in piglets, rats and sheep $[14,33,39]$. Furthermore, both experimental and clinical studies showed that post-asphyxia mild hypothermia is feasible and safe $[13,14,35]$. Several randomised clinical trial are currently in progress to test the efficacy and safety of post-asphyxia mild hypothermia. The mechanisms whereby hypothermia protects neurons against asphyxia are still not fully understood. Several studies demonstrated that hypothermia effects cerebral metabolism, decreases ATP depletion $[40,42]$ and reduces glutamate release $[3,16,25]$ during asphyctic-like insults. Furthermore, hypothermia is able to decrease free radical formation [19] and prevent post-ischemic caspase3 activation $[11,34]$.

To get more insight into the mechanisms underlying the neuroprotective effect of hypothermia, we investigated the effect of hypothermia on the stress gene response after perinatal asphyxia in the rat. Specifically, overproduction of $70 \mathrm{kDa}$ heat shock protein (hsp 70 ) has been shown to play an important role in the cellular defence mechanism that protect the brain against cell death produced by ischemia and other stresses [32]. Another gene important for neuronal function and survival is the glucose-regulated protein, GRP78, which is localized in the endoplasmic reticulum [18]. GRP78 is synthesized in all cells in response to low levels of glucose and oxygen. Changes in mRNA levels of hsp70, grp78 and XBP-1 (transcription factor essential for grp 78 activation) [6] were studied using semi-quantitative RT-PCR in homogenated brain tissue of rat pups with a history of perinatal asphyxia treated with or without hypothermia.

\section{Materials and methods}

\section{Perinatal asphyxia}

Eightteen full-term pregnant Wistar rats and their male pups $(n=20)$ were used (Charles River-Broekmans, Someren, The Netherlands). They were housed under standard conditions (12:12 $\mathrm{h}$ light:dark cycles, $20^{\circ} \mathrm{C}$ ) with free access to standard laboratory chow and water. The local Committee on Animal Welfare of the University of Maastricht approved all animal care 
and procedures.

Asphyxia was induced in rat fetuses at birth ( $\mathrm{P} 0$ ) by placing the uteri and its contents in a water bath for 19 minutes, as described in detail previously [37]. Briefly, dams were decapitated and rapidly hysterectomized immediately after delivery of two pups (control vaginal delivery; CVD). The uterus horns containing the remaining fetuses were detached and placed in a water bath at $37^{\circ} \mathrm{C}$ for exactly $19 \mathrm{~min}$ (severe perinatal asphyxia; SPA). The remaining pups were then removed from the uterine horns and stimulated to breath by cleaning the skin and by gently padding them on the chest. The maximal duration of resuscitation was 5 minutes. If a pup was not able to gasp by then, it was excluded from the study.

The CVD and SPA pups ( $\mathrm{n}=5$ per group per time point) were left to recover within a carefully controlled environment of $37^{\circ} \mathrm{C}$ and $90 \%$ humidity for $60 \mathrm{~min}$ with the use of a paediatric incubator. Hypothermia was induced by placing CVD and SPA pups in a paediatric incubator, set at room temperature $\left(21^{\circ} \mathrm{C} ; 90 \%\right.$ humidity), for $45 \mathrm{~min}$ (CVD-HYP, SPA-HYP; $n=5$ per group per time point). These pups were then placed with the normothermic pups (CVD and SPA) in a pediatric incubator set at $37^{\circ} \mathrm{C}$ for $15 \mathrm{~min}$. Finally, all surviving pups were randomly assigned to a surrogate mother ( 10 pups per mother) which had given birth on the same day. Mortality within the CVD and SPA group was $0 \%$ and $\pm 40 \%$, respectively. Hypothermia did not affect the mortality rate.

\section{Temperature measurements}

The temperature of the rat pups was measured orally using a special designed thermometer. Rat pups were kept in the pediatric incubator during the measurements and touched as little as possible. Temperature measurements were taken every ten minutes, starting $10 \mathrm{~min}$ after birth. As control, vaginally delivered pups that were born on the same day and who remained with their mother were measured during their first hours after birth using the same device.

\section{Collection of brain tissue and RT-PCR analysis}

Rat pups were randomly selected and rapidly decapitated at 6 and 24 hours after birth. The brain was rapidly removed from the skull and brain regions of interest (cerebellum, striatum, hippocampus and cerebral cortex) were dissected out and collected in RNAse free eppendorf tubes. The hippocampus samples were later discarded due to difficulties in dissecting at $6 \mathrm{hrs}$ and 24 hrs after birth. All tissue was weighed and rapidly frozen in liquid nitrogen. Samples were stored at $-70^{\circ} \mathrm{C}$ until processing.

Total RNA was isolated using the acid guanidinium thiocyanate/phenol/chloroform extraction method [7] and reverse transcribed into cDNA. Changes in mRNA levels of hsp 70, grp78, $\mathrm{xbp}-1$ were evaluated using quantitative PCR as described earlier [10,12]. Briefly, PCR reactions for hsp 70 and grp 78 were run in the presence of an internal standand, which had a non-matching sequence in the middle flanked by the sequences of the interest. Thus the internal standard could be amplified together with the specific cDNA using the same set of primers but yielding a smaller PCR product. The following pairs of primers were used: hsp 70, 5'- 
'TGCTGACCAAGATGAAGG-3" and 5'-AGAGTCGATCTCCAGGC-3'; GRP78, 5'GTTCTGCTTGATGTGTGTCC-3' and 5'-TTTGGTCATTGGTGATGGTG-3'; XBP-1, 5'AAACAGAGTAGCAGCGCAGACTGC-3' and 5'-GGATCTCTAAAACTAGAGGCTTGGTG-3'. The PCR products obtained with the XPB- 1 primers were further digested with PstI to reveal a restriction site that is lost after IRE 1 -mediated cleavage and splicing of the MRNA. IRE 1 is a stress-activated endonuclease resident in the ER and IRE-1 mediated cleavage controls expression of XBP-1 and GRP78 [6]. For evaluation, the PCR products were separated on a $2 \%$ agarose gel supplemented with ethidium bromide, gels were transilluminated with UV light and bands were photographed. Films were scanned with a rotating microdensitometer and the optical density of bands was evaluated by image analysis using appropiate standard curves, as previously described [12]. The standard curves were prepared by running PCR reactions with different concentration ratios of plasmids containing internal standard cDNA or the cDNA of interest as inserts.

\section{Statistical analysis}

All data are presented as mean \pm standard error of the mean (SEM). Statistical significant: differences between experimental groups were evaluated using a pairwise two way ANOVA analysis and post-hoc tests were performed using the Bonferroni t-test corrected for repeated measures. Statistics were carried out using SigmaStat ${ }^{i m}$ software version 2.03. Statistical significance was assumed to exist at $P=0.05$.

\section{Results}

\section{Temperature measurements}

Oral temperatures of the rat pups $(n=10$ per group) during the first $90 \mathrm{~min}$ of life are shown in Figure 1. CVD and SPA rat pups were subjected to normothermia $\left(37^{\circ} \mathrm{C}, 60 \mathrm{~min}\right)$ or hypothermia $\left(21^{\circ} \mathrm{C}, 45 \mathrm{~min}\right.$ and then $37^{\circ} \mathrm{C}, 15 \mathrm{~min}$ ) directly after birth (time of asphyxia). Oral temperatures of CVD-HYP and SPA-HYP rat pups were significantly decreased compared to the CVD and SPA groups during first 2 hrs of life $(\mathrm{P}<0.01$; minimum respectively 24.0 $\pm 0.3^{\circ} \mathrm{C}$ and $23.5 \pm 0.3^{\circ} \mathrm{C}$ at $50 \mathrm{~min}$ after birth). Oral temperatures of SPA-HYP were significantly reduced compared to the CVD-HYP group at 30 and 40 min after birth $(P<$ 0.05). At two hours after birth, onal temperatures of the rat pups subjected to hypothermia (between $33.0-34.2^{\circ} \mathrm{C}$ ) were comparable to the other pups (normothermic; see figure 1 ). Oral temperature of control rat pups, which after vaginal delivery remained with their mother, was approximately $34.7 \pm 0.2^{\circ} \mathrm{C}$ during the first $2 \mathrm{~h}$ of life. Ten min after birth, the temperature of control rat pups was approximately $27.6 \pm 0.5^{\circ} \mathrm{C}$, which increased during maternal nursing and/or physical activity. These data show that the oral temperature of vaginally delivered pups is much lower than that measured in more mature pups; after 3 days of nursing and feeding, the temperature of the control rat pups was approximately $35.8 \pm 0.4^{\circ} \mathrm{C}$. 


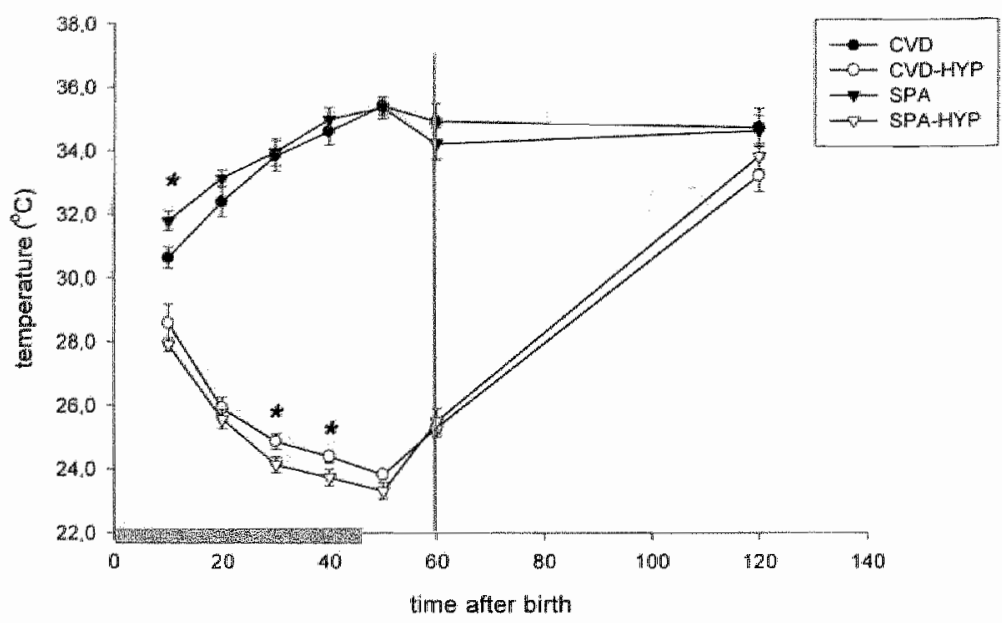

Figure 1. Oral temperature of rat pups during first $90 \mathrm{~min}$ after asphyxia. Rat pups were placed in a pediatric incubator, set at $37^{\circ} \mathrm{C}$ (normothermia) or $21^{\circ} \mathrm{C}$ (hypothermia) and $90 \%$ humidity. Rat pups were subjected to hypothermia for $45 \mathrm{~min}$; afterwards rat pups were placed within the pediatric incubator set at $37^{\circ} \mathrm{C}$ for $15 \mathrm{~min}$, before placing them with a surrogate mother. The gray bar indicates the time of hypothermia. The gray line indicates the time at which all pups were placed with a surrogate mother. The temperature of the CVD-HYP and SPA-HYP rat pups dropped respectively to $24.0^{\circ} \mathrm{C}$ and $23.5^{\circ} \mathrm{C}$ at 50 min after birth. At 90 min after birth, the temperature of all pups was comparable (between 33.0 $-34.2^{\circ} \mathrm{C}$ ). The oral temperature of CVD-HYP and SPA-HYP rat pups was significantly decreased compared to the temperature CVD and SPA during first 2 hrs of life $(P<0.01$; indicated by ${ }^{*}$ ). The temperature of SPA-HYP was significantly reduced compared to the temperature of CVD-HYP at 30 and 40 min after birth $(P<0.05)$.

\section{Gene response induced by asphyxia}

\section{mRNAs encoding for the cytoplasmic protein hsp 70}

Asphyxia-induced changes in striatal hsp $70 \mathrm{mRNA}$ levels are shown in Figure 2. Asphyxia caused $94 \%$ increase in hsp 70 mRNA levels at 24 hrs after the injury within the striatum ( $P=$ 0.05 ), whereas hsp 70 mRNA within the cerebellum was already $163 \%$ increased at 6 hrs after the injury $(p=0.04)$. Asphyxia did not affect hsp $70 \mathrm{mRNA}$ within the cerebral cortex at either 6 or 24 hrs after injury (birth).

mRNA levels of hsp 70 within the striatum of SPA rats were increased $(+59 \%)$ at 24 h after birth compared to 6 h after birth $(P=0.05)$. In contrary, hsp $70 \mathrm{mRNA}$ levels within the striatum of control rats were more stable $(-14 \%)$ during the first 24 hrs of life. Within the cerebellum of controls, hsp $70 \mathrm{mRNA}$ levels were $127 \%$ increased at 24 hrs after birth compared to 6 hrs after birth $(\mathrm{P}=0.04)$. mRNA of hsp 70 within the cerebellum of asphyctic rats at 24 here $85 \%$ decreased compared to hsp $70 \mathrm{mRNA}$ levels at $6 \mathrm{~h}$ after birth $(\mathrm{p}=0.04)$. mRNA levels of hsp 70 within the cortex of all rats remained stable during the first $24 \mathrm{~h}$ of life ( $6 \mathrm{~h}$ vs $24 \mathrm{~h}$ ). 


\section{mRNAs encoding for the ER-resident stress protein GRP 78 and XBP-I processing}

The patterns of change in $\mathrm{mRNA}$ levels of grp 78 after asphyxia within the striatum are shown in Figure 3. Grp $78 \mathrm{mRNA}$ levels within the striatum appeared to be reduced by the asphyxia at $6 \mathrm{~h}(-35 \%)$ and $24 \mathrm{~h}(-37 \%)$ after birth. These differences were, however, not significant $(\mathrm{P}>$ 0.05 ). Within the cerebellum, grp $78 \mathrm{mRNA}$ levels were unchanged by the asphyxial insult. Within the cortex, grp $78 \mathrm{mRNA}$ levels were slightly reduced at $6 \mathrm{~h}$ atter birth $(-65 \% ; \mathrm{P}=$ $0.05)$, but again restored at $24 \mathrm{~h}$ after birth.

Grp $78 \mathrm{mRNA}$ levels within the striatum were increased by $77 \%$ at $24 \mathrm{~h}$ after birth compared to $6 \mathrm{~h}$ after birth in control rats $(\mathrm{P}=0.04)$. Striatal grp $78 \mathrm{mRNA}$ levels in asphyctic rats were $72 \%$ increased at $24 \mathrm{hrs}$ compared to $6 \mathrm{~h}$ after birth $(\mathrm{P}=0.04)$. Within the cerebellum and cortex, grp 78 mRNA levels remained fairly stable during the first 24 h of life.

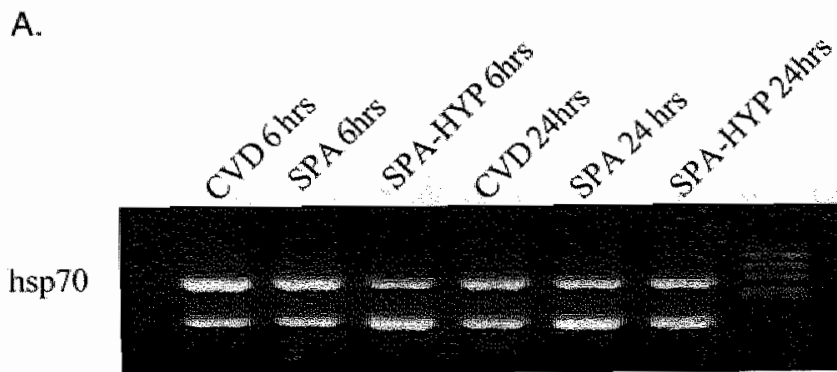

B.

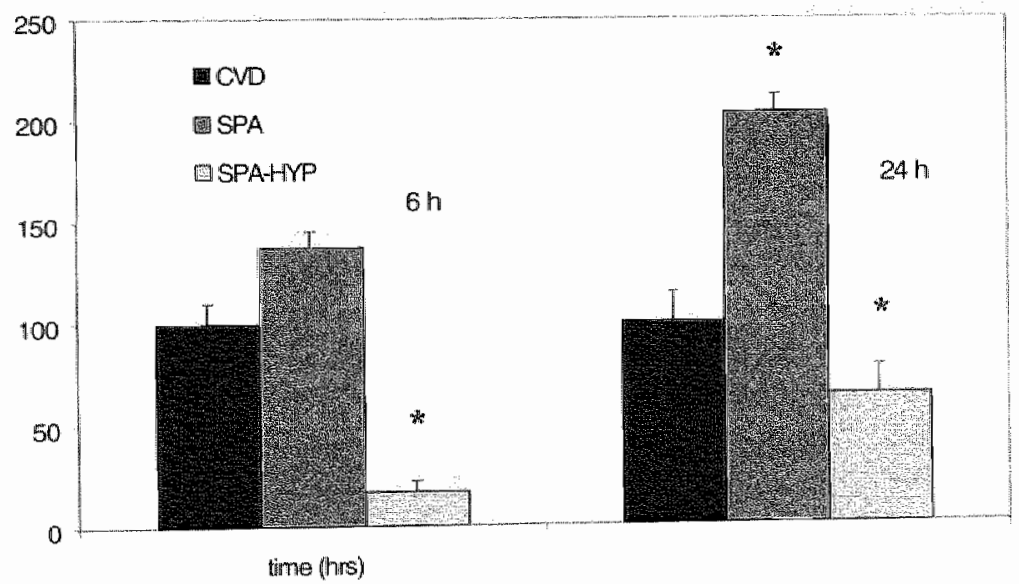

Figure 2. Changes in hsp70 mANA levels induced by asphyxia and hypothermia were evaluated by seimi-quantitative RT-PCR. Data are presented as percentage of control value. Striattal hsp70 mRNA levels were increased by $94 \%$ at $24 \mathrm{hrs}$ after the perinatal asphyxial injury. Striatal hsp70 mRNA levels were decreased after post-asphyxia hypothermia. (A) PCA products were separated on a agarose gel and changes in mRNA levels were assessed by image analysis $(B)$. ${ }^{*} p<0.05$ or $p=0.05$. 
A.

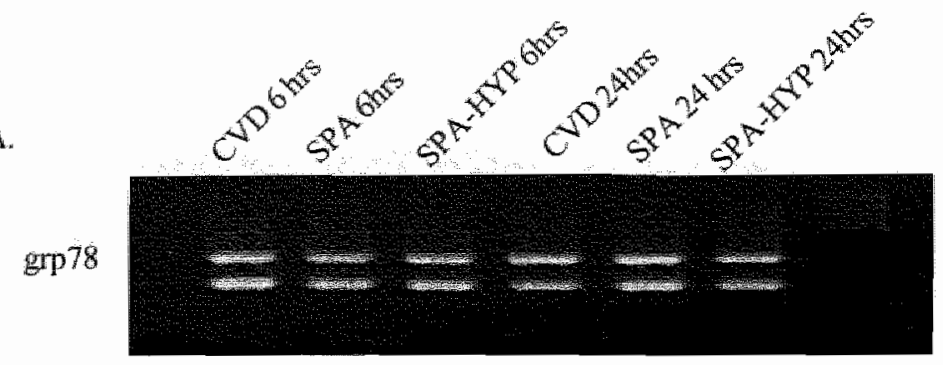

B.

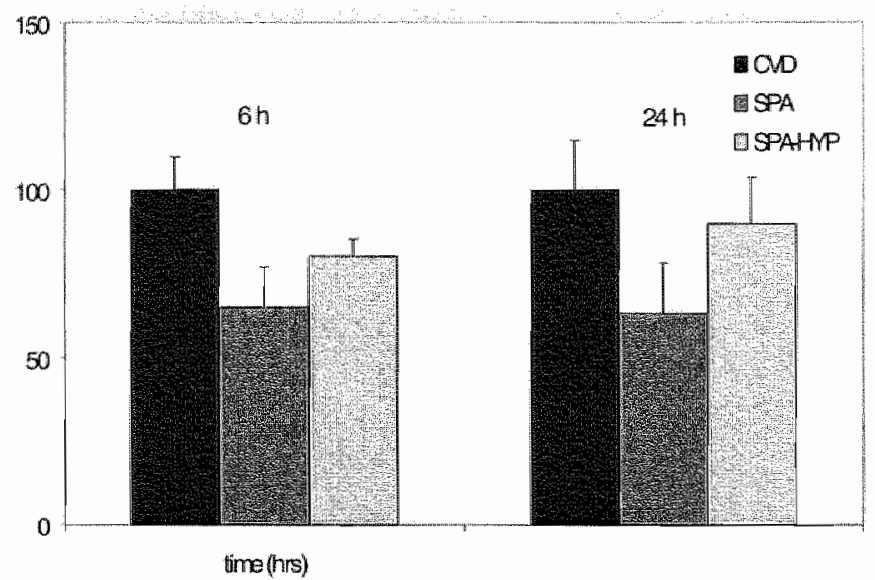

Figure 3. Changes in striatal grp78 mRNA levels induced by asphyxia and hypothermia were evaluated by seimi-quantitative RT-PCR. Data are presented as percentage of control. Striatal grp78 mRNA levels were not statistically changes after perinatal asphyxia or postasphyxia hypothermia. (A) PCR products were separated on a agarose gell and changes in $\mathrm{mRNA}$ levels were assessed by image analysis (B).

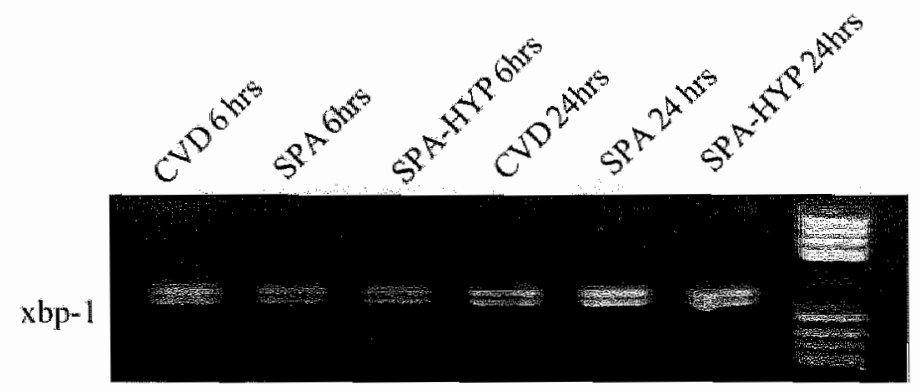

Figure 4. Effect of perinatal asphyxia and hypothermia on processing of XBP-1 mRNA in homogenated striatal tissue. Perinatal asphyxia did not induce XBP-1 processing. 
Activation of an ER stress response causes IRE 1-mediated splicing of a small intron from XBP-1 mRNA [6]. The protein encoded by the processed XBP-1 mRNA accumulates during the stress response and is required for the activation of target genes, including GRP78. Perinatal asphyxia did not induce XBP-1 processing at $6 \mathrm{~h}$ or $24 \mathrm{~h}$ after birth within the striatum, cerebellum or cortex (see Figure 4), indicating that there was no ER stress response induced by the asphyxial insult at $6 \mathrm{~h}$ or $24 \mathrm{~h}$ after birth.

\section{Gene response induced by post-asphyxia hypothermia}

\section{mRNAs encoding for the cytoplasmic protein hsp 70}

Post-asphyxia hypothermia prevented the increase in hsp $70 \mathrm{mRNA}$ levels caused by asphyxia within the striatum at $24 \mathrm{~h}$ after birth (SPA-HYP vs SPA; see Figure 2). Within the cerebellum, it prevented the increase in hsp $70 \mathrm{mRNA}$ levels at $6 \mathrm{~h}$ and the reduction at $24 \mathrm{~h}$. Within the cortex, hsp $70 \mathrm{mRNA}$ levels were stable after post-asphyxia hypothermia.

Post-asphyxia hypothermia caused a $72 \%$ decrease in hsp $70 \mathrm{mRNA}$ levels within the striatum at $6 \mathrm{~h}$ after birth, and a slight (-44\%) reduction at $24 \mathrm{~h}$ after birth (SPA-HYP vs CVD). Within the cerebellum and cortex, it did not affect hsp $70 \mathrm{mRNA}$ levels at $6 \mathrm{~h}$ or $24 \mathrm{~h}$ after birth. There was no difference in hsp $70 \mathrm{mRNA}$ levels between samples of CVD and CVD-HYP at any timepoint $(\mathrm{P}>0.05)$.

\section{$m R N A s$ encoding for $G R P 78$ and $X B P-1$ processing}

Post-asphyxia hypothermia did not affect grp $78 \mathrm{mRNA}$ levels within the striatum or cerebellum at 6 or $24 \mathrm{~h}$ after birth (Figure 3 ). It prevented the slight reduction in grp $78 \mathrm{mRNA}$ levels within the cortex at $6 \mathrm{~h}$ after birth. At $24 \mathrm{~h}$ after birth, grp $78 \mathrm{mRNA}$ levels in samples or SPAHYP were similar to grp 78 mRNA levels in control or asphyctic rats. Furthermore, postasphyxia did not affect processing of XBP-1 (see Figure 4).

\section{Discussion}

In this study, we investigated the effects of post-asphyxial hypothermia on the stress gene response in a rat model for intrauterine asphyxia at term. During the hypothermic period, the oral temperature of the rat pups dropped to $\pm 24^{\circ} \mathrm{C}$ and was maintained or less then $34^{\circ} \mathrm{C}$ for a 90 min period. Our results demonstrate that the induction of hsp $70 \mathrm{mRNA}$ as a consequence of perinatal asphyxia is strongly influenced by body temperature during the first few hours after injury. Post-asplayxia hypothermia prevented and suppressed the injury induced expression of hsp 70 mRNA, suggesting that hypothermia prevented cytoplasmic stress. Perinatal asphyxia did not affect grp 78 mRNA levels or XBP-1 processing, suggesting that ER function was preserved after this injury. Furthermore, our results indicate that post-asphyxia hypothermia did not induce changes GRP78 mRNA levels or XBP-1 processing, indicating that the 
hypothermic period allone did not affect ER function.

Previous studies demonstrated that post-insult hypothermia is effective in preventing brain damage following intrauterine asphyxia [14,39]. In our study, we applied cooling immediately after the cerebral insult and continued for $45 \mathrm{~min}$. This paradigm was chosen to prevent glucose and maternal deprivation; and all pups were placed with a nursing dam within 60 min of the injury. Furthermore, long-term neuroprotection has been obtained when hypothermia was induced immediately after injury and prolonged for hours [5]. However, such prolonged cooling may also increase the risk of serious side effects. The optimal period and setting of hypothermia for neonates has yet to be investigated in randomized clinical trials. The present study represents a mechanistic study in regard to the impact of perinatal asphyxia and post-asphyxial hypothermia on stress gene response after injury.

Currently, it is unclear which mechanisms are responsible for the delayed cell death induced by perinatal asphyxia, as described recently $[8,27,37]$. Evidence has been provided for mitochiondrial failure and ER dysfunction leading to activation of caspases and eventually cell death (see $[15,22]$ for reviews). Heat shock proteins (HSPs) play a vital role in normal cellular function during postnatal devellopment [17] and serve as chaperones, which bind to other proteins and aid the restoration of the structure and function of denatured proteins. Thus, the induction of heat shock proteins represents a cellular defense mechanism that may protect the cell against any stress that induces protein denaturation, including asphyxia. A number of studies have shown that HSP70 is part of a cytoplasmic stress response system which can protect cells against both necrotic and apoptotic cell death [32]. HSP70 has been shown to protect against glutamate-mediated toxicity [20], protect astrocytes against acidosis-produced damage [26] and inhibit cytochrome C-mediated Capase-9 and -3 activation [2,23]. In the present study, we show that overproduction of hsp 70 mRNA levels is induced within $24 \mathrm{~h}$ of the insult within both the striatum and cerebellum, but not the cerebral cortex. Previously, we have shown that the striatum and cerebellum are particularly vulnerable to perinatal asphyxia mediated cell loss [37]. Taken together, these data show that hsp 70 overproduction was induced within the damaged brain regions during the first $24 \mathrm{~h}$ of life, possible in a neuroprotective function. Future studies are required to test whether prolonged overproduction of hsp 70 can provide further neuroprotection and prevent excess cell death.

Disturbances in ER function also induce a stress response, which activates the expression of genes encoding for transcription factors (e.g. XBP-1) and ER-resident proteins (e.g. grp78, grp94 and gadd 153) and kinases resulting in the suppression of global protein synthesis $[4,28]$. GRP78 is known to act as molecular chaperone regulating protein folding and facilitating protein translocation in the endoplasmic reticulum and protein secretion. Our results show that perinatal asphyxia did not induce any changes in grp $78 \mathrm{mRNA}$ levels during the first $24 \mathrm{~h}$ after the insult. This suggests that ER function was preserved following perinatal asphyxia and that cell death was primarily caused by cytoplasmic stress. Previous studies have shown that cerebral ischemia, induced via carotid artery occlusion, can induce ER dysfunction and changes in the expression of genes coding for ER-resident stress proteins $[29,30]$. This insult induces both necrotic and apoptotic cell death within the damaged regions. In contrary, the perinatal asphyxia paradigm used in this study does not induce necrotic cell death, but only apoptotic 
cell death, which peaks at postnatal day (P) 8 [37]. Furthermore, the amount of cell death induced by the cerebral ischemic insult is much larger than amount of cell death detected after perinatal asphyxia $[8,37]$. Therefore, we conclude that the ER dysfunction is not responsible for the delayed apoptotic cell death following perinatal asphyxia. Changes in the expression of genes coding for ER-resident proteins, however, might predict the severity of the insult and the amount of subsequent cell death.

The resuits of the present study show that post-asphyxia hypothermia can suppress hsp 70 overproduction induced by perinatal asphyxia. These data confirm previous studies showing that the expression of hsp $70 \mathrm{mRNA}$ is strongly influenced by temperature. It has been suggested that the expression of hsp $70 \mathrm{mRNA}$ after perinatal asphyxia is directly related to the intensity of cellular stress [1]. The decrease in hsp $70 \mathrm{mRNA}$ levels following post-asphyxia hypothermia may then be explained as a decrease in cellular stress and eventually in cell death. However, our results might also indicate that the drop in temperature during the cooling period was too large, leading to impaired energy metabolism and subsequently reduced transcriptional activity. The consequence of a reduction in transcriptional activity on brain function and injury recovery remains unknown. Future studies are necessary to investigate the long-term consequences of post-asphyxia hypothermia and of the effects of temporary suppression of global transcriptional activity.

Following hypothermia, the regional pattern of expression of grp 78 mRNA within the striatum, cerebellum and cortex was unchanged. Furthermore, XBP-1 processing was not affected by hypothermia. These data indicate that post-asphyxia hypothermia did not cause ER stress or effect transcriptional activity of these genes. Therefore, we conclude that perinatal asphyxia causes cytoplasmic stress during the first $24 \mathrm{~h}$ after birth, which may result in cell injury. Cytoplasmic stress after perinatal asphyxia can be prevented using mild post-asphyxia hypothermia. 


\section{References}

[1] Aoki, M., Tamatani, M., Taniguchi, M., Yamaguchi, A., Bando, Y, Kasai, K, Miyoshi Y., Nakamura, Y., Vitek, M.P., Tohyama, M. Tanaka, H. Sugimoto, H. 2001. Hypothermic ureatment restores glucose regulated protein 78 (GRP78) expression in ischemic brain. Mol Brain Res. 95.

[2] Beere, H. M., Wolf, B.B., Cain, K., Mosser, D.D., Mahboubi, A., Kuwana, T., Tailor, P., Morimoto, R.I. Cohen, G.M., Green, D.R. 2000. Heat-shock protein 70 inhibits apoptosis by preventing recruitment of procaspase-9 to the Apaf-1 apoptosome. Nat Cell Biol. 2: 469-75.

[3] Berger ${ }_{i 1}$., Jensen, A., Hossmann, K., Paschen W. 1998. Effect of mild hypothermia during and after transient in vitro ischemia on metabolic disturbances in hipocampal slices at different stages of development. Dev Brain Res. 105: 67-77.

[4] Bodsch, W., Takahashi, K., Barbier, A., Grosse Ophoff, B. Hossmann, K.-A. 1985. Cerebral protein synthesis and ischemia. Prog Brain Res. 63: 197-210.

[5] Bona, E., Hagberg, $H_{\text {, }}$ Loberg, E.M., Banasiak, K., Thoresen, M. 1998. Protective effects of moderate hypothermia after neonatal hypothermia after neonatal hypoxia-ischemia: shortand long-term outcome. Pediatr Res. 43: 738-745.

[6] Calfon, M., Zeng, H., Urano, F., Till, J.H., Hubbard, S.R., Harding, H.P., Clark, S.G., Ron . D. 2002. IRE1 couples endoplasmic reticulum load to secretory capacity by processing the XBP-1 mRNA. Nature. 415: 92-96.

[7] Chomoczynski, P., Sacchi, N. 1987. Single-step method of RNA isolation by acid guanidinium thiocyanate-phenol-chloroform extraction. Anal Biochem. 162: 156-159.

[8] Dell'Anna, E., Chen, Y., Engidawork, E., Andersson, K., Lubec, G., Luthman, J. and HerreraMarschitz, M. 1997. Delayed neuronal death following perinatal asphyxia in rat. Exp Brain Res. 115: 105-15.

[9] Dietrich, W. D., Busto, R., Alonso, O., Globus, M.Y, ginsberg, M.D. 1993. Intraischemic but not postischemic brain hypothermia protects chronically following global forebrain ischemia in rats. J Cereb Blood Flow Metab. 13: 541-549.

[10] Doutheil, J., Althausen, S., Gissel, C., Paschen, W. 1999. Activation of MYD116(gadd34) expression following transient forebrain ischemia of rat: implications for a role of disturbances of endoplasmic reticulum calcium homeostasis. Mol Brain Res. 63: 225-232.

[11] Fukuda, $H_{\text {. }}$ Tomimatsu, T., Watanabe, N., Mu, J.W., Kohzuki, M., Endo, M., Fujiil, E., Kanzakj, T. and Murata, Y. 2001. Post-ischemic hypothermia blocks caspase-3 activation in the newborn rat brain after hypoxia-ischemia. Brain Res. 910: 187-91.

[12] Gissel ${ }_{\text {, }}$., Doutheil, J., Paschen, W. 1997. Temporal analysis of changes in neuronal c-fos mRNA levels induced by dpeletion of endoplasmic reticulum calcium stores $\ddot{O}$ effect of clamping cytoplasmic calcium activity at resting levels. J Neurochem. 69: 2538-2545.

[13] Gunn, A. J. 2000. Cerebral hypothermia for prevention of brain injury following perinatal asphyxia. Curr Opin Pediatr. $12: 111-5$.

[14] Gunn, A. J., Gunn, T.R., Gunning M.I, de Haan, H.H., Williams, C.E., Gluckman, P.D. 1997. Dramatic neuronal rescue with prolonged selective head cooling after ischemia in fetal lambs. J Clin Invest. 99: 248-256.

[15] Inder, T. E. and Volpe, J. J. 2000. Mechanisms of perinatal brain injury. Semin Neonatol. 5: 316.

[16] Li, P. A., He, Q. P., Miyashita, H., Howllet, W.s Siesjo, B. K. and Shuaib, A. 1999. Hypothermia ameliorates ischemic brain damage and suppresses the release of extracellular amino acids in both normo- and hyperglycemic subjects. Exp Neurol. 158: 242-53.

[17] Lindquist, S. Craig, E.A. 1988. The heat shock proteins. Annu Rev Genet. 22: 631-677.

[18] Little, E., Ramakrishnan, M., Roy, B., Gazit, G., Lee, A.S. 1994. The glucose-regulated 
proteins (GRP78 and GRP94): functions, gene regulation, and applications. Critical reviews in Eukaryotic gene expression. 4: 1-18.

[19] Loidl, C. F., De Vente, J., van Ittersum, M. M., van Dijk, E. H., Vles, J. S., Steinbusch, H. W. and Blanco, C. E. 1998. Hypothermia during or after severe perinatal asphyxia prevents increase in cyclic GMP-related nitric oxide levels in the newborn rat striatum. Brain Res. 791: 303-7.

[20] Lowenstein, D. H., Chan, P.H., Miles, M.F. 1991. The stress protein response in cultured neurons: characterization and evidence for a protective role in excitotoxicity. Neuron. 7: 1053-1060.

[21] Mallard, E. C., Williams, C. E., Johnston, B. M. and Gluckman, P. D. 1995. Neuronal damage in the developing brain following intrauterine asphyxia. Reprod Fertil Dev. 7: 647-53.

[22] Mishra, O.P. and Delivoria-Papadopoulos, M. 1999. Cellular mechanisms of hypoxic injury in the developing brain. Brain Res Bull. 48:233-8.

[23] Masser, D. D., Caron, A.W., Bourget, L., Meriin, A.B. ,Sherman, M.Y., Morimoto "R.I., Massie, B. 2000 . The chaperone function of hsp70 is required for protection against stress-induced apoptosis. Mol Ceil Biol. 20:7146-59.

[24] Nakamura, Y., Nakashima, T., Fukuda, S., Nakashima, H. and Hashimolo, T. 1986. Hypoxicischemic brain lesions found in asphyxiating neonates. Acta Pathol Jpn. 36: 551-63.

[25] Nakashima, K. and Todd, M. M. 1996. Effects of hypothermia on the rate of excitatory amino acid release after ischemic depolarization. Stroke. 27: 913-8.

[26] Narasimhan, P., Swanson, R.A., Sagar, S.M., Sharp, F.R. 1996. Astrocyte survival and HSP7O heat shock protein induction following heat shock and acidosis. Glia. 17: 147-159.

[27] Northington, F. J., Ferriero, D. M., Flock, D. L. and Martin, L. J. 2001. Dellayed neurodegeneration in neonatal rat thalamus after hypoxia-ischemia is apoptosis. J Neurosci. 21: $1931-8$.

[28] Paschen, W.2000. Role of calcium in neuronal cell injury: which subcellular compartment is involved? Brain Res Bull. 53: 409-413.

[29] Paschen, W., Doutheil, J. 1999. Disturbances of the functioning of endoplasmic reticulum: a key mechanism underlying neuronal cell injury? J Cereb Blood Flow Metab. 19: 1-18.

[30] Paschen, W., Gissel, C., Linden, T., Althausen, S., Doutheill, J. 1998. Activation of gadd153 expression through transient cerebral ischemia: evidence that ischemia causes endoplasmic reticulum dysfunction. Mol Brain Res. 60: 115-122.

[31] Pasternak, J.F., Predey, T. A. and Mikhael, M. A. 1991. Neonatal asphyxila: vulnerability of basal ganglia, thalamus, and brainstem. Pediatr Neurol. 7: 147-9.

[32] Sharp, F. R., Massa, S.M., Swanson, R.A. 1999. Heat-shock protein protection. TINS. 22: 97-99.

[33] Thoresen, M., Penrice, J., Lorek, A. Cady, E.B., Wylezinska, M., Kirkbride, V., Cooper" C.E., Brown, G.C., Edwards, A.D., Wyatt, J.S. et al. 1995. Mild hypothermia after severe transient hypoxia-ischemia ameliorates delayed cerebral energy failure in hte newborn piglet. Pedriatr Res. 37.

[34] Tomimatsu, T., Fukuda, H., Endo, M., Watanabe, N., Mu. J., Kohzuki, M., Fujii, E., Kanzaki, $T$. and Murata, Y, 2001. Effects of hypothermia on neonatal hypoxic-ischemic brain injury in the rat: phosphorylation of Akt, activation of caspase-3-like protease. Neurosci Lett. 312: 21-4.

[35] Trescher, W. H., Ishiwa, S. and Johnston, M. V. 1997. Brief post-hypoxic-ischemic hypothermia markedly delays neonatal brain injury. Brain Dev. 19: 326-38.

[36] Trescher, W. H. Ishiwa, S., Johnston, M.V. 1997. Brief post-hypoxic-ischemic hypothermia markedly delays neonatal brain injury. Brain Dev. 19: 326-338.

Van de Berg, W. D., Schmitz, C., Steinbusch, H. W. and Blanco, C. E. 2002. Perinatal 
asphyxia induced neuronal loss by apoptosis in the neonatal rat striatum: a combined TUNEL and stereological study. Exp Neurol. 174: 29-36.

[38] Volpe, J. J. 2001. Perinatal brain injury: from pathogenesis to neuroprotection. Ment Retard Dev Disabil Res Rev. 7: 56-64.

[39] Wagner, B. P. Nedelcu, J. and Martin, E. 2002. Delayed postischemic hypothermia improves long-term behavioral outcome after cerebral hypoxia-ischemia in neonatal rats. Pediatr Res. 51:354-60.

[40] Williams, G. D., Dardzinski, B. J., Buckalew, A. R. and Smith, M. B. 1997. Modest hypothermia preserves cerebral energy metabolism during hypoxia-ischemia and correllates with brain damage: a $31 \mathrm{P}$ nuclear magnetic resonance study in unanesthetized neonatal rats. Pediatr Res. 42: $700-8$.

[41] Vager, J., Towfighi, J., Vannucci, R.C. 1993. Influence of mild hypothermia on hypoxic-ischemic brain damae in the immature rats. Pediatr Res. 34: 525-529.

[42] Yager, J. Y. Asselin, J. 1996. Effect of mild hypothermia on cerebral energy metabolism during the evolution of hypoxic-ischemic brain damage in the immature rat. Stroke. 27: 919926. 
General discussion 


\section{Introduction}

The studies presented in this thesis show that global asphyxia at term has long-lasting effects on the number of neurons and synapses within specific regions of the brain. The striatum was most severely affected by the injury. Global asphyxia caused an increase in developmental apoptosis within the striatum, which peaked at P8, and loss of both GABergic projection neurons and inteneurons at 2 months of age. In addition, our data indicate that global asphyxia at term causes both motor and cognitive deficits in later life. Finally, we show that hypothermia can directly affect the stress response during the first $24 \mathrm{~h}$ after injury. The relevance of these results, their clinical application and future directions will be discussed in this chaper.

\section{Rat model for perinatal asphyxia}

The studies presented in this thesis were performed using a rat model developed by Bjelke and co-workers [1]. This model was chosen for the studies since it represents a non-invasive experimental model that induces global asphyxia (whole body asphyxia) at term, in contrary to the Rice-Vannucci model that induces focal hypoxia-ischemia during the postnatal development of the rat (see chapter 1). Moreover, the global asphyxia model is well suited for studying long-term neurological and behavioural deficits after acute perinatal asphyxia. A disadvantage of the global asphyxia model is the high and variable mortality in the asphyxia group (40-80\%). Important concerns in this regard include: "Does the high mortality cause a selection-bias (survival of the fittest)? And why is the mortality so variable?" We think that early mortality is due to cardiovascular failure and not brain mediated. This is supported by the finding that cell death after perinatal asphyxia occurs within hours and peaks at 8 days after the insult (chapter 3 and 4 ). Therefore, death due to brain cell loss would more likely occur within hours or days after the insult. In addition, the variability in mortality is likely due to variability in litter size, brain maturation stage at the time of birth/insult as well as maternal weight and strain.

One of the major concerns regarding the global asphyxia model and its possible relevance to human is the age at which the injury is induced. There is no consensus on a postmatal age at which the rat brain could reflect the human brain at term. The grow spurt of the human brain peaks around birth and of the rat brain around postnatal day 7 [2]. For this reason, many researchers used the 7-day-old rat Rice-Vannucci model as a model of perinatal asphyxia in the human brain. Considering the formation of synapses within the rat brain which peaks at 4 6 weeks [15], it could even be more relevant to study asphyxia at a much later stage [18]. Thus, one could argue that the global asphyxia model reflects the situation of a premature infant with a history of asphyxia more closely than a term infant. It is important to note that the timing of developmental processes, namely synaptogenesis, neurogenesis, gliagenesis, myelination, apoptosis among others, and growth spurt is different between species. The underlying mechanisms, i.e. apoptosis, however seem to be resemble the human situation. 
Evidence for Caspase-3 activation and caspase-like proteolytic activity for example has been provided in postmortem brain tissue of infants who suffered acute perinatal asphyxia [14]. Therefore, we conclude that studies using this global asphyxia model should focus on molecular and/or biochemical mechanisms underlying brain damage as well as the long-lasting behavioural effects.

\section{Cell death as a consequence of global asphyxia at term}

Acute asphyxia causes selective damage within the developing white matter in the premature infant prior to 32 weeks of gestation. The vulnerability of the grey matter in the brain, especially the basal ganglia and cerebral cortex, increases towards term. The global asphyxia model reflects rather closely the clinical features of acute asphyxia in neonates in which lesions are characterically found in the basal ganglia and thalamus $[12,16]$. The dorsolateral part of the striatum was most severely affected by the perinatal brain injury. This might indicate that the striatal cells are more vulnerable to excitotoxic damage and oxidative stress at this age than cells in other brain areas. The striatum, hippocampus and cerebral cortex contain large number of glutamate receptors $[9,20]$. Excitotoxicity can occur when glutamate or aspartate causes excessive amounts of calcium and sodium to traverse neuronal membranes [22]. Selective vulnerability in the developing brain can partially be explained by understanding the age and anatomically specific expression patterns of glutamate receptors. Microinjection of the glutamate agonist NMDA into postnatal rats demonstrated that hypoxicischemic brain injury is enhanced at 7 days of age in rats, compared to older or younger pups [9]. The density of NMDA receptors is also higher in the early postnatal period of rats than in adulthood $[9,10,19]$, suggesting that there is an overshoot in the number of receptors followed by pruning later in development. Several other factors could potentially contribute to the selective vulnerability of GABAergic medium spiny neurons and GABAtergic intemeurons within the striatum. It is possible that the additional vulnerability of these cells arises fiom the enormous metabolic expenditure of GABAergic medium spiny neurons required to maintain transmembrane potentials and/or interaction with additional factors including local NO release (chapter 5), the distribution of calcium-binding cells or local densities of trophic factors [11].

White matter lesions ranging from subtle alterations with focal necrosis to almost total infarction have been described in fetal sheep brain exposed to different times of intrauterine asphyxia $[3,5]$. In contrast, hypoxia-ischemia induced during postnatal development (P7) in the rat (Rice-Vannucci model) causes grey rather than white matter damage. Apoptotic cell death after global asphyxia in the term rat is not restricted to neuronal death within the grey matter. Oligodendrocytes and glial cells within the corpus callosum and white matter of cerebellum are also damaged by the insult (chapter 4). Moreover, global asphyxia at term has been shown to produce long-lasting but subtle myelination deficits within the corpus 
callosum, hippocampus and cerebellum [8]. Thus altogether, these results provide evidence for both short and long-term grey and white matter changes after global asphyxia in the term rat. This animal model is therefore an interesting and powerful tool to study structural relationships between white and grey matter damage, a subject that is still controversial in the pathophysiology of perinatal asphyxia.

Glial cells play an important role in the regulating of neurogenesis and neuronal fate [17]. This suggests that the loss of glial cells may affect neuronal proliferation as well as neuronal survival. Loss of glials due to asphyxia may therefore lead to a loss of mature neurons and reduced neurogenesis. There is also evidence that white matter damage in the premature infant has a profound adverse effect on subsequent neuronal development within the cerebral cortex [6]. This suggests that the loss of oligodendrocytes during postnatal development can have an adverse effect on neuronal survival. Therapeutic strategies should therefore not only focus on neuronal survival, but cell survival in general.

\section{Long-term consequences of global asphyxia at term}

Infants exposed to moderate or severe perinatal asphyxia typically develop motor impairments, for example cerebral palsy and/or cognitive impairments [21]. The studies in this thesis show that global asphyxia in the term rat reduces motor activity and slightly impaires walking pattern at 2 months of age (chapter 6). Furthermore, long-term cognitive performance was impaired at 18 months of age in rats with a history of global asphyxia at term (chapter 8). Although the differences between the asphyctic and control rats were rather small, these data show that global asphyxia in the term rat has long-lasting effects on behavioural function.

Our results indicate that global asphyxia at term leads to both neuronal, glial and synaptic loss at 2 months of age within the striatum (chapter 6 and 7). It is generally accepted that the functional outcome after injury is related to synaptic numbers $[13,23]$. So, it is reasonable to assume that a decrease in synaptic number impairs behavioural function. The striatum plays a central role in motor function and injury to the striatum is often associated with cerebral palsy [4]. Little is known however about the specific structural basis of this disorder. Thus, treatment strategies that are able to stimulate synaptic growth and plasticity could be beneficial for improving functional outcome of infants with cerebral palsy.

\section{Therapeutic strategies}

Several therapeutic strategies have been proposed for perinatal asphyxial brain injury and few are being tested. Advances in the understanding of the mechanisms present during and after asphyxia have prompted the use of therapies oriented to post-asphyxia mild hypothermia. Concerning the effectiveness and the protective effect of post-asphyxia hypothermia, no 
conclusions can be drawn from the studies in this thesis. This is partly due to the short time frame in which the gene response was investigated (chapter 5). Another limitation of the experimental setup was that we were unable to control body temperature of the rat pups. Body temperature of the pups at room temperature dropped to $24^{\circ} \mathrm{C}$. This temperature drop might have been too large, since mild hypothermia $\left(\sim 33^{\circ} \mathrm{C}\right)$ is more effective than severe hypothermia in animal experiments. In our study, post-asphyxial hypothermia reduced the post-asphyxial increase in hsp70. This suggests that post-asphyxia hypothermia inhibits endogenous neuroprotective mechanisms or that it prevents the cellular stress response. This observation should be further studied in the near future.

Interestingly, the temperature measurements of asphyctic and control rats indicate that body temperature of asphyctic rats was lower than control rats during the first 30 min of life, which could suggest that an endogenous neuroprotective strategy is activated after asphyxia. This might partly explain why these rats are so highly resistant to a global asphyxial insult at term.

\section{Conclusions and future perspective}

The studies in this thesis illustrate that increased NO release and Caspase activation play an important role in the initiation of cell death following perinatal asphyxia. These data provide a basis for the mechanism involved in brain injury after perinatal asphyxia and the development of therapeutic agents that could be able to improve functional outcome. Future experiments on the consequences of perinatal asphyxia in the rat should involve both short-and long-term evaluation of biochemical parameters in order to evaluate functional implications of the brain injury. In addition, determination of the interactions between and/or response of different neurotransmitter systems to the loss of GABAergic neurons within the striatum during development would allow more insight in the basis of neurological deficits after perinatal asphyxia.

Most studies in the literature focus on mechanisms of cell death after brain injury. The studies in this thesis show that it is also important to understand the mechanisms of cell survival during development and aging. Therapeutic strategies may be more beneficial when they are able to improve neuronal and synaptic plasticity as well as protect against cell death. Learning and physical activity can improve functional outcome after perinatal asphyxia [7, 13], likely via the induction of both neuro-, glia- and synaptogenesis. Consequently, studying molecular mechanisms involved in learning and physical activity as well as neuro- and synaptogenesis may elucidate whether these therapeutic strategies could improve the outcome of infants with a history of perinatal asphyxia and which therapeutic strategies should be used. A therapy combining hypothermia with anti-apoptotic and pro-plasticity agents may be a promising strategy to improve long-term functional outcome. Hypothermia is able to extend the therapeutic window as well as limit cellular damage (short-term). Treatment with proplasticity agents, such as growth factors, environmental enrichment including socialisation, may in turn stimulate the surving cells to make more synaptic connections and improve longterm functional outcome. 


\section{References}

[1] Bjelke, B., Andersson, K. Ogren, S.O. and Bolme, P. 1991. Asphyctic lesion: proliferation of tyrosine hydroxylase-immunoreactive nerve cell bodies in the rat substantia nigra and functional changes in dopamine neurotransmission. Brain Res 543: 1-9.

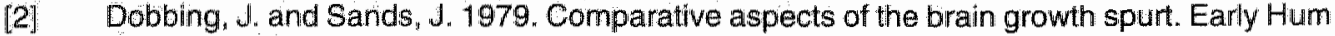
Dev 3: 79-83.

[3] Falkowski, A, Hammond, R., Han, V. and Richardson, B. 2002 Apoptosis in the preterm and near term ovine fetal brain and the effect of intermittent umbilical cord occlusion. Brain Res Dev Brain Res 136: 165-73.

[4] Hayashi, M., Satoh, J., Sakamoto, K. and Morimatsu, Y. 1991. Clinical and neuropathological findings in severe athetoid cerebral palsy: a comparative study of globo-Luysian and thalamoputaminal groups. Brain Dev 13: 47-51.

[5] Ikeda, T., Choi, B.H., Yee, S., Murata, Y. and Quilligan, E.J. 1991. Oxidative stress, brain white matter damage and intrauterine asphyxia in fetal lambs. Int $J$ Dev Neurosci 17: 1-14.

[6] Inder, T.E., Huppi, P.S., Wartield, S., Kikinis, R., Zientara, G.P., Barnes, P.D., Jolesz, F. and Volpe, J.J. 1999. Periventricular white matter injury in the premature infant is folllowed by reduced cerebral cortical gray matter volume at term. Ann Neurol 46: 755-60.

[7] Kim, H.T., Kim, I.H., Lee, K.J., Lee, J.R., Park, S.K., Chun, Y.H., Kim, H. and Rhyu, I.J. 2002. Specific plasticity of parallel fiber/Purkinje cell spine synapses by motor skill learning. Neuroreport 13: 1607-10.

[8] Kohlhauser, C., Mosgoller, W., Hoger, H. and Lubec, B. 2000. Myelination deficits in brain of rats following perinatal asphyxia. Life Sci 67: 2355-68.

[9] McDonald, J.W. and Johnston, M.V. 1990. Physiological and pathophysiological roles of excitatory amino acids during central nervous system development. Brain Res Brain Res Rev 15: 41-70.

[10] McDonald, J.W., Johnston, M. V. and Young, A.B. 1990. Differential ontogenic development of three receptors comprising the NMDA receptor/channel complex in the rat hippocampus. Exp Neurol 110: 237-47.

[11] Mitchell, I.J. "Cooper , A.J. and Griffiths, M.R. 1999. The selective vulnerability of striatopallidal neurons. Prog Neurobiol 59: 691-719.

[12] Pasternak, J.F. and Gorey, M.T. 1998. The syndrome of acute near-total intrauterine asphyxia in the term infant. Pediatr Neurol 18: 391-8.

[13] Ramirez-Amaya, V., Balderas, I., Sandoval, J., Escobar, M.L. and Bermudez-Rattoni, F. 2001. Spatial long-term memory is related to mossy fiber synaptogenesils. J Neurosci 21 : $7340 \sim 8$.

[14] Rossiter, J.P. Anderson, L.L., Yang, F. and Cole, G.M. 2002. Caspase-3 activation and caspase-like proteolytic activity in human perinatal hypoxic-ischemic brain injury. Acta Neuropathol (Berl) 103:66-73.

[15] Shimohama, S., Fujimoto, S., Sumida, Y., Akagawa, K., Shirao, T., Matsuoka, Y. and Taniguchi, T. 1998. Differential expression of rat brain synaptic proteins in development and aging. Biochem Biophys Res Commun 251: 394-8.

[16] Sie, L.T., van der Knaap, M.S., Oosting, J., de Viries, L.S., Lafeber, H.N. and Valk, J. 2000. MA patterns of hypoxic-ischemic brain damage after prenatal, perinatal or postnatal asphyxia. Neuropediatrics 31: 128-36.

[17] Song, H., Stevens, C.F. and Gage, F.H. 2002. Astroglia induce neurogenesis from adult neural stem cells. Nature 417: 39-44.

[18] Terry, R.D. and Katzman, R. 2001. Life span and synapses: will there be a primary senile dementia? Neurobiol Aging 22: 347-8; discussion 353-4. 
[19] Tremblay, E., Roisin, M.P., Represa, A., Charriaut-Marlangue, C. and Ben Ar, Y, 1988, Transient increased density of NMDA binding sites in the developing ral hippocamops. Brain Res 461: 393-6.

[20] Trescher, W.H., McDonald, J.W. and Johnston, M.V. 1994 Qunolinate-induced migny is enhanced in developing rat brain. Brain Res Dev Brain Res 83: 224-32.

[21] Volpe, J.J. 1998. Brain injury in the premature infant: overview of clinical aspects, neuropathology, and pathogenesis. Semin Pediatp Neurol 5: 135-51.

[22] White, R.J. and Reynolds, I.J. 1995. Mitochondria and Na+/Ca2+exchange buther glutamateinduced calcium loads in cultured cortical neurons. J Neurosoi 15: 131828.

[23] Wong, T.P., Campbell, P.M., Ribeiro-da-Silva, A. and Cuello, A.C. 1998. Synaptic numbers across cortical laminae and cognitive performance of the rat during aging. Neuroscience 84: $403-12$. 
.204 


\section{Summary}

The major aims of the present thesis "Cell death and synaptic remodelling as a consequence of perinatal asphyxia. Implications of hypothermia" were to investigate cellular processes induced by perinatal asphyxia that lead to cell death, the long-term consequences of perinatal asphyxia and the neuroprotective effects of hypothermia following perinatal asphyxia.

The studies show that in a rat model of perinatal asphyxia neurons, glial cells and oligodendrocytes die mainly from apoptotic mechanisms, involving Caspase- 3 activation, production of nitric oxide and cytoplasmic stress. The main cell types that die in the striatum are the GABAergic projection neurons and parvalbumin-immunoreactive interneurons. Synaptic density was altered after perinatal asphyxia within the striatum and cortex, both two months and 22 months of age. Behavioural assessment reveals minor motor deficits at 6 weeks of age and age-related memory impairment at 22 months of age. Post-asphyxia hypothermia appears to reduce the cytoplasmic stress response during the first 24 hours after injury (birth).

In chapter 1, an overview of the current state of knowledge on perinatal asphyxia and cell death in rats, including the cellular mechanisms leading to cell death and nitric oxide production, is given. Asphyxia and hypoxia-ischemia are defined, brain regions with respect to neurotransmitters are introduced and regeneration and behavioural outcome after perinatal asphyxia is reviewed. In particular, consequences and (dis)advantages of the use of the RiceVannucci model (unilateral carotid ligation followed by hypoxia) and the global asphyxia model (intra-uterine hypoxia-ischemia) are discussed. Finally, therapeutic strategies to reduce brain injury following perinatal asphyxia are discussed.

In summary, hypoxia-ischemia (Rice-Vannucci model) produces mild or severe focal lesions depending on the duration of hypoxia and age of induction. Global asphyxia does not produce a focal lesion, but damage in several brain regions, including the striatum, thalamus and cortex. Hypoxia-ischemia (Rice-Vannucci model) at P7 induces both necrotic and apoptotic cell death, mainly within the grey matter. Global asphyxia produces cell death within both the grey and white matter. The cells die mainly from apoptotic mechanisms. It was concluded that both rat models are able to resemble certain clinical features of perinatal asphyxia, such as motor deficits and/or cognitive impairment. Furthermore, there seems to be a direct relationship between the extent of structural damage within specific brain regions and the functional deficits as a consequence of the perinatal brain injury. Post-asphyxia hypothermia has proven to be effective in animal models and non-toxic in infants. Therefore, we concluded that postasphyxia hypothermia is one of the most promising therapies currently available. Furthermore, it can provide a therapeutic window in which caspase inhibitors or anti-inflammatory agents can act.

In chapter 2, methodological issues concerning stereological assessment of the total number of cells in frozen tissue from rat and human brain are discussed. We addressed the question whether or not sections of snap-frozen rat brain may be used for counting neurons using the 
optical disector. Frozen cryostat sections from hippocampal and cerebellar regions of two rat strains and cerebellar and cerebral regions from human brain were analysed using the optical disector. The results did not differ from data reported previously in the literature. Therefore, it was concluded that cryostat sections of snap-frozen tissue can successfully be applied for estimating total neuronal numbers using the optical disector.

In chapter 3, cell damage within the striatum following perinatal asphyxia was determined using a quantitative neuro-anatomical approach. Double stranded DNA breaks were visualized using the TUNEL method. Morphological changes were studied using Haematoxylin-Eosin staining and Hoechst, in conjunction with confocal microscopy. Cell loss within the striatum was assessed by using state-of-the-art stereology. It was concluded that perinatal asphyxia leads to delayed cell death (up to 15 days of the injury) in the striatum, with morphological features of apoptosis. This lead to a $16 \%$ reduction in the total number of neurons within the rat striatum at three weeks of age.

In chapter 4 , the ontogeny of apoptotic cell death during postnatal development after perinatal asphyxia was studied in the striatum, hippocampus and cerebellum. The objective was to characterize, using qualitative methods, which cell type is particularly vulnerable to a perinatal asphyxial insult and whether these cells exhibited morphological features of apoptosis and/or necrosis. In addition, the time course of Caspase-3-like activity after perinatal asphyxia was assessed (quantitative analysis). Apoptosis was examined by TUNEL, immunohistochemistry using an antibody against Caspase-3, electron microscopy and Caspase-3-like activity assay. In summary, perinatal asphyxia increased the number of TUNEL-positive cells within the striatum and hippocampus, but did not affect the distribution of the dying cells within these areas. TUNEL-positive cells could partly be identified as astrocytes and oligodendrocytes. Perinatal asphyxia increased Caspase-3-like activity in the hippocampus at $\mathrm{P} 8$ and caused a decrease at $P 11$ compared to control pups. No changes were found in the subventricular zone and the cerebellum. It was concluded that perinatal asphyxia primarily causes delayed apoptotic cell death within the proliferating areas of the striatum and hippocampus.

In the study presented in chapter 5, NO production following perinatal asphyxia during the first 8 days a fter birth was investigated. Intracellular NO was measured using a fluorometric method based on the reaction of DAF-2 with intracellular NO, yielding the highly fluorescent derivative $\mathrm{DAF}-2$ triazole. The study provides evidence for a large increase in DAF-2/NO production and cGMP levels in the striatum in the first 8 days after the perinatal asphyxial insult without marked astrogliosis. These results support the hypothesis that NO plays an important role in mediating cellular damage in the striatum after perinatal asphyxia. Furthermore, the results show that perinatal asphyxia triggers a long-lasting reaction leading to an increase in NO synthesis up to 8 days after the insult.

The aim of chapter 6 was to investigate whether behavioural deficits following perinatal asphyxia are related to striatal damage. Behavioural performance of control rats and rats 
exposed to perinatal asphyxia was tested using the open field task, grip task and foot print task at three and 6 weeks of age. At eight weeks of age, the number of calbindin- and parvalbuminimmunoreactive neurons within the rat striatum were determined using stereology and the binding activity of the GABAA receptor in de different projection areas of the striatum was studied using receptor autoradiography. It was shown that perinatal asphyxia, in the global asphyxia rat model, results in minor changes in spontaneous behaviour. A decrease in locomotor behaviour during aging was found in the control group, whereas the rats exposed to perinatal asphyxia showed a comparable and relatively low spontaneous activity at three and 6 weeks of age. An increase in stride width after asphyxia, indicative of a disturbance in balance or stability when walking, was found in the foot print task. The total number of calbindin- $22 \%$ ) and parvalbumin-immunoreactive $(-43 \%)$ neurons within the striatum were decreased after perinatal asphyxia at eight weeks of age, whereas the binding activity of the postsynaptic GABAA receptor within the striatum and its projection areas was unaffected by the asphyxial insult. It was concluded that perinatal asphyxia may result in minor deficits in motor performance despite a considerable loss in both GABAergic projection neurons and interneurons within the striatum. Furthermore, loss of striatal neurons after perinatal asphyxia does not necessarily affect GABAA receptor binding activity within the striatum or its projection areas.

The aim of the study presented in chapter 7 was to establish whether there are region-specific changes in synaptic numbers after perinatal asphyxia. The total number of neurons and the density of synaptophysin-immunoreactive presynaptic boutons (SIPB) within the striatum, $\mathrm{CA} 1$ and CA3 layer of the hippocampus, and cerebellum of control rats and rats exposed to perinatal asphyxia were investigated using quantitative methods considering stereologic principles. The study shows that perinatal aspliyxia primarily affect the dorsomedian and dorsocaudal part of the striatum. The number of pyramidal neurons within the CA1-CA3 regions was reduced in rats exposed to perinatal asphyxia at three weeks of age, whereas synaptic density was unchanged at two months of age. Perinatal asphyxia did not affect the total number of Purkinje cells within the cerebellum but the synaptic density within the molecular layer of the cerebellum was slightly reduced. It was concluded that perinatal asphy xia causes regionspecific damage and synaptic loss primarily within the rat forebrain.

In chapter 8, the long-term consequences of perinatal asphyxia are discussed. Cognitive performance and synaptic density following perinatal asphyxia was investigated at respectively 18 and 22 months of age. The spatial Morris water escape task did not reveal short-term memory of learning deficits in old rats exposed to perinatal asphyxia compared to old control rats. Perinatal asphyxia did cause long-term memory impairment ( 18 months old rats versus 1.5 months old rats) and an increase in presynaptic bouton density in the parietal contex at 22 months of age. Presynaptic bouton density was unchanged in the striatum and/or frontal cortex in rats exposed to perinatal asphyxia compared to control rats. An increase in striatal volume was observed in rats exposed to perinatal asphyxia. It was concluded that perinatal asphyxia leads to augmented age-related cognitive impairment and an increase in the total number of presynaptic boutons within the parietal cortex and striatum. 
The aim of the study presented in chapter 9 was to investigate the response of the cytoplasmic stress-related gene hsp 70 and the endoplasmic reticulum genes grp 78 and $x b p-1$ after perinatal asphyxia and post-asphyxia hypothermia. Hsp 70 was increased in the striatum at $24 \mathrm{~h}$ after asphyxia and this response was prevented by post-asphyxia hypothermia. It contrast, grp-78 and $x b p-1$ processing were not affected by perinatal asphyxia. This study provides evidence for cytoplasmic stress after perinatal asphyxia rather than endoplasmic reticulum stress. Furthermore, the results show that post-asphyxia hypothermia is able to prevent the cytoplasmic stress response and does not induce endoplasmic stress.

\section{Final conclusions}

The great advantage of the global asphyxia rat model is that it induces global asphyxia in the fetus at the time of birth and allows for long-term survival and behavioural analysis. It was concluded that studies using the global asphyxia model should focus on studying molecular and/or biochemical mechanisms underlying brain damage as well as the long-lasting behavioural effects. Furthermore, the rat model is an interesting and powerful tool to study the structural relationships between white and grey matter damage as global asphyxia causes cell death in both the white and grey matter during the postnatal period. Therapeutic strategies should not only focus on neuronal survival, but cell survival in general. Furthermore, therapeutic strategies that are able to stimulate synaptic growth and plasticity might be beneficial for improving outcome of infants with a history of perinatal asphyxia. 


\section{Samenvatting}

Het doel van de studies in dit proefschrift, getiteld "Cell death and synaptic remodelling as a consequence of perinatal asphyxia. Implications of hypothermia" was het bestuderen van (1) cellulaire processen, geïnduceerd door perinatale asfyxie, die leiden tot celdood; (2) de langetermijneffecten van perinatale asfyxie; (3) en de neuroprotectieve effecten van hypothermie na asfyxie.

De studies in dit proefschrift tonen aan dat in een diermodel voor perinatale asfyxie neuronen, glia cellen en oligodendrocyten doodgaan als gevolg van apoptotische mechanismen, waarbij Caspase-3 activiteit, de productie van stikstof monooxide en cytoplasmatische stres een belangrijke rol spelen. Voornamelijk sterven GABAerge projecterende neuronen en parvalbumine-immunoreactieve interneuronen in het striatum als gevolg van perinatale asfyxie. Perinatal asfyxie verandert ook de dichtheid van de synapsen in het striatum en de cortex. Functionele studies toonden aan dat perinatale asfyxie kleine motorische afwijkingen en aanveroudering-gerelateerde geheugenafwijkingen kan veroorzaken. Hypothermie na asfyxie kan cytoplasmatische stress reduceren gedurende de eerste 24 uur na letsel (geboorte).

In hoofdstuk 1 is een overzicht gegeven van de huidige kennis op het gebied van perinatale asfyxie en celdood in ratten. De cellulaire mechanismen die tot celdood en productie van stikstof monooxide leiden, zijn besproken en de termen asfyxie en hypoxie-ischemie zijn gedefinieerd. Tevens zijn verschillende hersengebieden en neurotransmitter geïntroduceerd, evenals regeneratieprocessen en de gevolgen van perinatale asfyxie op gedrag. In het bijzonder zijn de gevolgen en voor-en/of nadelen van het Rice-Vannucci diermodel voor perinatale asfyxie (unilaterale ligatie van de carotis gevolgd door hypoxie) en het globale asfyxie model (in uterus hypoxie-ischemie) besproken. Ten slotte zijn therapeutische behandelingsstrategieën ter vermindering van langetermijneffecten van perinatale asfyxie belicht.

Hypoxie-ischemie (Rice-Vannucci model) produceert milde of ernstige focale laesies. De grootte van de laesie is afhankelijk van duur van hypoxie en de leeftijd waarop de asfyxie wordt geïnduceerd. Globale asfyxia produceert geen focale laesie, maar veroorzaakt schade in verschillende hersengebieden, zoals het striatum, de thalamus en de cortex. Hypoxieischemie (Rice-Vannucci model), geinduceerd op P7, leidt tot necrotische en apoptotische celdood, voornamelijk in de grijze stof. Global asfyxie veroorzaakt celdood in de grijze en witte stof. De cellen gaan dood als gevolg van apoptotische mechanismen. Beide diermodellen kunmen klinische kenmerken van asfyxie nabootsen, zoals motorische afwijkingen en/of cognitieve stoornissen. Tevens, blijkt er een directe relatie te bestaan tussen de structurele schade in specifieke delen van de hersenen en functionele afwijkingen veroorzaakt door perinatale asfyxie. Er is aangetoond dat hypothermie na asfyxie effectief kan zijn in diermodellen en niet toxisch is voor pasgeborenen. Derhalve, concluderen wij dat hypothermie na asfyxia één van de meest veelbelovende therapieën is. Hypothermie kan het schadeproces vertragen en verschaft zo een mogelijkheid om met behulp van caspase remmers en ontstekingsremmers de schade te beperken. 
In hoofdstuk 2 worden methodologische kwesties die betrekking hebben op stereologische bepalingen van het totale aantal cellen in bevroren materiaal van hersenen afkomstig van ratten of mensen, belicht. De vraag of coupes van bevroren rattenhersenen gebruikt kunnen worden woor het kwantificeren wan neuronen met behulp van de 'optical disector' stond centraal. Bevroren coupes, gemaakt met een cryostaat, van de hippocampus en cerebellum van twee verschillende rattenstammen en van humane hersenen werden geanalyseerd met behulp van de 'optical disector'. De resultaten waren vergelijkbaar met eerder gepubliceerde data. Derhalve concludeerde wij dat cryostaat coupes van diep-gevroren materiaal gebruikt kan worden voor de bepaling van het totale aantal neuronen met de 'optical disector'.

In hoofdstuk 3 werd celdood in het striatum als gevolg van perinatale asfyxia bestudeerd door middel van een kwantitatieve neuro-anatomische aanpak. Dubbelstrengse DNA breuken werden gevisualiseerd met behulp van de TUNEL methode. Morfologische veranderingen werden bestudeerd door middel van aankleuring met Hematoxyline-Eosine of Hoechst, in samenwerking met confocale microscopie. Het aantal verloren cellen in het striatum werd bepaald met behulp van stereologie. De resultaten van deze studie laten zien dat perinatale asfyxia tot vertraagde celdood (tot 15 dagen na de geboorte) in het striatum kan leiden. De degenererende cellen vertoonden apoptotische kenmerken. De celdood leidde tot een reductie in het totale aantal neuronen in het striatum, 3 weken na de geboorte.

In de studie beschreven in lhoofdstuk $\mathbf{4}$ werd de tijdscurve van apoptotische celdood tijdens postnatale ontwikkeling na perinatale asfyxie in het striatum, de hippocampus en het cerebellum bestudeerd. Het doel van de studies was het bepalen van het typen cellen gevoelig voor asfyxie op een kwalitatieve wijze. Tevens werd gekeken of deze cellen apoptotische of necrotische kenmerken vertoonden. De mate van Caspase-3 activiteit na perinatale asfyxie werd gemeten (kwantitatieve analyse). Apoptotische kenmerken werden met behulp van TUNEL, immunohistochemie met antilichamen tegen Caspase-3, elektron microscopie, en Caspase-3 activiteitsassay bestudeerd.

Perinatale asfyxia leidde tot een toename van het aantal TUNEL-positieve cellen in het striatum en de hippocampus, maar had geen affect op de distributie van de degenererende cellen in deze gebieden. TUNEL-positieve cellen konden gedeeltelijk geïdentificeerd worden als astrocyten en oligodendrocyten. Perinatale asfyxie veroorzaakte een toename in Caspase-3 activiteit in de hippocampus op P8 en een afname op P11. Er werden geen veranderingen gemeten in de regio rondom het ventrikel en het cerebellum. Perinatal asfyxie veroorzaakt dus primair vertraagde apoptotische celdood in gebieden van het striatum en de hippocampus waar proliferende cellen aanwezig zijn.

In de studie gepresenteerd in hoofdstuk 5 werd het effect van perinatale asfyxie op de productie van stikst of monooxide $(\mathrm{NO})$ onderzocht. Intracellulaire $\mathrm{NO}$ werd gemeten door middel van een fluorometrische methode, gebaseerd op de reactie van DAF-2 met intracellulair NO en resulterend in het sterk fluorescerende product DAF-2 triazole. De studie toont aan dat 
perinatale asfyxie een grote toename in DAF-2/NO productie en cGMP concentraties in het striatum veroorzaakt gedurende de eerste 8 dagen na de geboorte zonder dat er astrogliose optreedt. Deze resultaten ondersteunen de hypothese dat NO een belangrijke rol speelt bij het induceren van cel schade in het striatum na asfyxie. Tevens laten de resultaten zien dat perinatale asfyxie een langdurende reactie teweegbrengt die leidt tot een toename van NO synthese.

Het doel van de studie in hoofdstuk 6 was het bestuderen van functionele afwijkingen die veroorzaakt zijn door perinatale asfyxie en gerelateerd zijn aan schade in het striatum. Het gedrag van controle ratten en ratten die blootgesteld waren aan perinatale asfyxie werd geèvalueerd door middel van de open veld test, grip test en voet-afdruk-test, 3 en 6 weken na de geboorte. Het totale aantal calbindin- en parvalbumine-immunoreactieve neuronen in het striatum werd bepaald met behulp van stereologie. De bindingsactiviteit van de GABAA receptor in het striatum, de globus pallidus en substantia nigra werd bestudeerd met behulp van receptor autoradiografie.

Perinatale asfyxie leidde tot kleine afwijkingen in spontaan motorische gedrag. Een afname in motorische activiteit tijdens veroudering ( 3 versus 6 weken leeftijd) werd gemeten bij controle ratten, terwijl ratten die blootgesteld waren aan perinatale asfyxie een vergelijkbare en relatief lage activiteit vertoonden op 3 en 6 weken leeftijd. Een toename in de stap-afstand werd gevonden in de voet-afdruk test, hetgeen duidt op een verstoring van de balans of stabiliteit tijdens lopen. Het totale aantal calbindin- $(-22 \%)$ en parvalbumine-immunoreactieve ($43 \%$ ) neuronen in het striatum was afgenomen als gevolg van perinatale asfyxie op 2 maanden leeftijd. De bindingsactiviteit van de postsynaptische GABAA receptor in het striatum, de globus pallidus en de substantia nigra was niet aangedaan door perinatale asfyxie. Perinatale asfyxie kan dus kleine motische afwijkingen veroorzaken ondanks een groot verlies in het aantal GABAerge projecterende neuronen en interneuronen in het striatum. Tevens doen deze resultaten vermoeden dat verlies van striatale neuronen niet noodzakelijkerwijs de bindingsactiviteit van de GABAA receptor beinvloedt.

Het doel van de studie in hoofdstuk 7 was vaststellen of er regionale veranderingen in het aantal synapsen optreden na perinatale asfyxie. Het totale aantal neuronen en de dichtheid van synaptophysin-immunoreactieve presynaptische boutons (SIPB) in het striatum, de CA1 en CA3 laag van de hippocampus, en het cerebellum van controle ratten en ratten blootgesteld aan perinatale asfyxie werd bestudeerd met behulp van kwantitatieve methoden gebaseerd op stereologische principes. De studie toont aan dat perinatale asfyxie primair het dorsomediane en dorsocaudale deel van het striatum beschadigt. Het aantal pyramidale cellen in het CA1CA3 gebied was gereduceerd in ratten die blootgesteld waren aan perinatale asfyxie, terwijl de dichtheid van de SIPB onveranderd was op twee maanden leeftijd. Perinatale asfyxie had geen effect op het totale aantal Purkinje cellen in het cerebellum, maar de dichtheid van de SIPB was licht gereduceerd. Er kan dus geconcludeerd worden dat perinatal asfyxie regiospecifieke schade en verlies van SIPB induceerd, voornamelijk in het voorste deel van de hersenen. 
Om meer inzicht te verkrijgen in de langetermijneffecten van perinatale asfyxie werd in hoofdstuk 8 de cognitieve prestatie en de dichtheid van SIPB van controle ratten en ratten blootgesteld aan perinatale asfyxie gemeten op respectievelijk 18 en 22 maanden. De spatiële 'Morris water escape' test liet geen kortetermijneffecten van perinatale asfyxie op het geheugen of de leerprestatie zien op 18 maanden leeftijd. Perinatale asfyxie veroorzaakte langetermijneffecten op cognitieve prestatie ( 18 maanden oude ratten versus 1,5 maanden oude ratten) en een toename in de dichtheid van SIPB in de parietale cortex op 22 maanden leeftijd. De dichtheid van SIPB was onveranderd in het striatum en de frontale cortex in ratten blootgesteld aan perinatale asfyxie. Een toename in het volume van het striatum werd gevonden bij ratten blootgesteld aan perinatale aspfyxie, vandaar dat er geconcludeerd kan worden dat perinatale asfyxie kan leiden tot een afname van de cognitieve prestatie tijdens veroudering en een toename van het totale aantal SIPB in de parietale cortex en het striatum op late leeftijd.

Het doel van de studie in hoofdstuk 9 was het bestuderen van het effect van perinatale asfyxie en hypothermie post-asfyxie op de concentratie van het cytoplasmatische stres-gerelateerde gen hsp 70 en de endoplasmatisch reticulum genen grp 78 en xbp-1. Hsp70 was toegenomen in het striatum 24 uur na de geboorte en deze response kon voorkomen worden door post-asfyxie hypothermie. In tegenstelling, de concentratie van grp 78 en het activeren van xbp-1. was niet veranderd door perinatale asfyxie. De studie toont aan dat perinatale asfyxie cytoplasmatische stress veroorzaakt en geen endoplasmatische stress. Tevens blijkt dat post-asfyxie hypothermie de cytoplasmatische stress kan voorkomen en zelf geen stress veroorzaakt.

\section{Eindconclusies}

Een groot voordeel van het globale asfyxie model is dat het globale asfyxie induceert in de foetus tijdens de geboorte en dat langetermijneffecten en functionele veranderingen bestudeerd kunnen worden. Studies gebruikmakend van het globale asfyxie model zouden zich moeten richten op het bestuderen van moleculaire en biochemische mechanismen betrokken bij celdood en het bestuderen wan langetermijneffecten. Het diermodel is een interessant en krachtig hulpmiddel om de relatie tussen structurele afwijkingen in de witte stof en grijze stof te bestuderen, aangezien globale asfyxie celdood veroorzaakt in beide gebieden. Therapeutische behandelingsstrategieën moeten zich niet alleen richten op de overleving van neuronen, maar op de overleving van cellen in het algemeen. Therapeutische behandelingsstrategieën die synaptische groei en plasticiteit kunnen stimuleren kunnen gunstige effecten hebben op de langetermijneffecten van perinatale asfyxie. 


\section{Curriculum vitae}

Wilma van de Berg was born on the first of March 1974. After passing the VWO- $B$ exam at the St. Maartenscollege in Maastricht, she studied Chemistry at the Catholic University of Nijmegen for one year. Afterwards, she studied Biomedical Health Science at the University of Maastricht. As a trainee, she performed a research project on Parkinson's disease and cell death at the department of Neuropsychology and Psychiatry of the University of Maastricht. Thereafter, she further specialized in Neuroscience at the Vision 2000 lab of the department of Neurobiology, Dalhousie University in Halifax (Canada). Here, she studied c-Jun phosphorylation after BDNF and GDNF infusion in a rat model for Parkinson's disease and was supervised by Prof. T. Hagg. In 1998, she received the Organon Young Research Award for her thesis 'Cell death: suicide or murder?' After graduation, she started a $\mathrm{PhD}$ project at the department of Pediatrics in cooperation with the department of Cellular Neuroscience at the University of Maastricht en University Hospital Maastricht (Research Institutes GROW and Brain \& Behaviour) in 1998. She studied the short-term and long-term consequences of perinatal asphyxia and was supervised by Prof. C.E. Blanco (department of Pediatrics) en Prof. H.W.M. Steinbusch (department of Cellular Neuroscience). The studies presented in this thesis were performed.

Wilma van de Berg werd geboren op 1 maart 1974. Zij behaalde haar VWO-B examen aan het St. Maartenscollege te Maastricht en ging Scheikunde studeren aan de Katholieke Universiteit Nijmegen voor 1 jaar. Vervolgens studeerde zij Biologische Gezondheidskunde aan de Universiteit van Maastricht. Tijdens haar stage voerde zij een studie uit naar Parkinson en celdood bij de vakgroep Neuropsychologie en Psychiatrie van de Universiteit van Maastricht. Daarna specialiseerde zij zich verder in de Neurowetenschappen in het Vision 2000 laboratorium van de vakgroep Neurobiologie van de Dalhousie universiteit te Halifax (Canada). Hier onderzocht zij, onder supervisie van Prof. dr. T. Hagg, c-Jun fosforylatie na BDNF en GDNF infusies in a diermodel voor Parkinson. In $1998 \mathrm{kreeg}$ zij voor haar afstudeerwerkstuk getiteld 'Cell death: suicide or murder' de Organon Young Research Award. Na haar afstuderen, werd ze aangesteld als assistent in opleiding (AIO) bij de vakgroep Kindergeneeskunde en de vakgroep Cellulaire Neurowetenschappen van de Universiteit van Maastricht en het Academisch Ziekenhuis Maastricht (onderzoeksinstituten GROW en Hersenen \& Gedrag). In dit kader heeft zij onderzoek verricht naar de korte- en langetermijneffecten van perinatale asfyxie onder leiding van Prof. dr. C.E. Blanco (vakgroep Kindergeneeskunde) en Prof. dr. H.W.M. Steinbusch (vakgroep Cellulaire Neurowetenschappen), hetgeen resulteerde in dit proefschrift. 


\section{Publications}

\section{First author}

W.D.J. Van de Berg, A. Blokland, A.C. Cuello, C. Schmitz, W. Vreuls, H.W.M. Steinbusch, C.E. Blanco. 2000. Perinatal asphyxia results in changes in presynaptic bouton number in striatum and cerebral cortex-a stereological and behavioral analysis. J Chem Neuroanat. 20:71-82.

W.D.J. van de Berg, C. Schmitz, H.W.M. Steinbusch, C.E. Blanco. 2002. Perinatal asphyxia induced neuron loss by apoptosis in the neonatal rat striatum: a combined TUNEL and stereological study. Experimental Neurology 174:29-36

W.D.J. Van de Berg, M. Kwaijtaal, A.J.A. De Louw, N.P.A. Lissone, C. Schmitz, R.L.M. Faull, A. Blokland, C.E. Blanco, H.W.M. Steinbusch. 2003. Impact of perinatal asphyxia on GABAergic and motor system. Neuroscience 117:83-96

W.D.J. Van de Berg, H.P.J. Steinbusch, L. Veerbeek, M. Huiban, M.O. Lopez-Figueroa, J. DeVente, C.E. Blanco, H.W.M. Steinbusch. In Press. Direct imaging of nitric oxide production using DAF-2/DA after perinatal asphyxia in the rat striatum and cerebellum. Eur. J.Neuroscience

W.D.J. Van de Berg, A. Scheepens, H.P.J. Steinbusch, H.W.M. Steinbusch, C.E. Blanco. In press. Qualitative and quantitative analysis of the ontogeny of apoptotic cell death during postnatal development after birth asphyxia. Experimental Neurology

W.D.J. Van de Berg, C. Schmitz, H.P.J. Steinbusch, J. Dortmans, J.S.H. Vles, H.W.M. Steinbusch, C.E. Blanco. Regional specificity in neuronal and synaptic loss after perinatal asphyxia in the rat brain (submitted).

W.D.J. Van de Berg, W. Paschen, A. Scheepens, H.W.M. Steinbusch, C.E. Blanco. Hypothermia post-asphyxia prevents cytoplasmic stress gene response induced by perinatal asphyxia (submitted). 


\section{Co-author}

D. Terwel, W.D.J. Van de Berg. 2000. c-Jun/AP-1 (N) directed antibodies cross-react with "apoptosis-specific autophagic process during neuronal apoptosis. Neuroscience 96: 445-6.

C. Schmitz, M. Dafotakis, H. Heinsen, K. Mugrawer, A. Niesel, G.J. Popken, M. Stephan, W.D.J. Van de Berg, S. von Horsten, H. Korr. 2000. Use of cryostat sections from snap frozen nervous tissue for combining stereological estimates with histological, cellular, or molecular analyses on adjacent sections. J Chem Neuroanat. 20:21-29.

A.J.A. De Louw, W.D.J. Van de Berg, J. De Vente, H.W.M. Steinbusch, A.W.D. Gavilanes, H.P.J. Steinbusch, J. Troost, J.S.H. Vles. 2001. Developmental apoptosis in the spinat cord white matter in neonatal rats. Glia 37:89-91.

M. Mulder, B.J A. Janssen, W.D.J. Van de Berg, L.M. Havekes, E.R. De Kloet, A. Blokland. Impaired memory and less hippocampal presynaptic boutons in low-density lipoprotein receptor-knockout mice (submitted).

A. Scheepens, G. Wassink, M.J. Piersma, W.D.J. Van de Berg, C.E. Blanco. In press. A delayed increase in hippocampal neurogenesis after severe global asphyxia in the neonatal rat.

Brain Res.

B.P.F. Rutten, O. Wirths, W.D.J. Van de Berg, T. Hartmann, G. Multhaup, S.F. Lichtentaler, J.Vehoff, H.W.M. Steinbusch, H.Korr, K. Beyreuther, T.A. Bayer, C. Schmitz. In Press. Expression of the $\beta$-cleaved C-terminal APP fragment does not cause loss of hippocampal neurons or alterations in hippocampal synaptic bouton numbers in aged transgenic mice. Neurobiol. of disease

B. Scholtissen, R. Deumens, W.D.J. Van de Berg, W. Honig, H.P.J. Steinbusch, A.F.G. Leentjens, A. Blokland, H.W.M. Steinbusch, J. Prickaerts. Functional investigations into the role of dopamine and serotonin in rats with partial bilateral striatal 6 hydroxydopamine lesions (submitted). 


\section{Dankwoord}

Dit proefschrift is tot stand gekomen dankzij de enthousiaste inzet en steun van vele mensen. De laatste woorden van dit proefschrift wil ik gebruiken om een aantal mensen te bedanken.

Prof. dr. C.E. Blanco, beste Carlos. In de afgelopen jaren heb ik je leren kennen als een fantastische mentor. Ik wil je bedanken voor je wetenschappelijke en vriendschappelijke steun. Je wist telkens weer de juiste kritische vragen te stellen, als ik met allemaal nieuwe, wilde ideeën en plannen in je deurpost stond. Ik wil je bedanken voor je steun bij mijn internationale escapades. Ik heb veel geleerd van de internationale congressen en werkbezoeken. Ik zal de gezamenlijke reizen missen. Bedankt voor je vertrouwen in mij en ik hoop dat we volgend jaar weer samen in de kroeg staan tijdens Carnaval.

Prof. dr. H.W.M. Steinbusch, beste Harry. Bedankt dat je me de mogelijkheid bood om in jouw lab mijn/onze ideeën uit te werken. De vakgroep 'Cellulaire Neurowetenschappen' fungeerde voor mij als een soort thuisbasis. Mede dankzij jou, heb ik me hier altijd 'thuis' gevoeld. Je deur stond en stalat altijd open en je gaf me de kans om nieuwe technieken te leren. Ik heb groot respect voor de manier waarop je EURON hebt opgezet. EURON geeft AIO's de kans om cursussen en werkbezoeken, zowel nationaal als internationaal, te doen. Ik wil je bedanken voor de mogelijkheid om specifieke technieken aan de universiteit te Aken en het Max-Planck instituut Keulen te leren en om deel te nemen aan internationale cursussen.

De analisten van het lab, beste Hellen Steinbusch en Marjanne Markerink. Het was erg fijn om met jullie samen te werken op het lab. Hellen, je hebt me laten zien hoe leuk het kan zijn om coupes te bekijken onder de microscoop en typen cellen te herkennen. We hebben veel gelachen tijdens uren achter de cryostaat en bij het opslepen van coupes. Bedankt voor al je inzet en je grote interesse voor mijn onderzoek. Marjanne, je hebt een groot hart en staat altijd voor iedereen klatar. Bedankt voor je luisterend oor en je vele adviezen. Wiel Honig, ik wil jou bedanken voor de ondersteuning bij de gedragsexperimenten en het image analyse (SIS) systeem en je humor.

Het asphyxia-team. Tijdens mijn promotie periode heb ik menige uren doorgebracht in de operatickamer met Fabian Loidl, Danilo Gavilanes, Anton de Louw, Hans Vles, Arjan Scheepens en Hellen Steinbusch en vele studenten, waaronder Willem Vreuls, Neirude Lissone, Judith Dortmans, Laetitia Veerbeek, Martijn Kwaijtaal en Monica Huiban. Ik wil jullie allen bedanken voor de enthousiaste samenwerking. We hebben veel gediscussieerd en gelachen tijdens het wachten op de geboorte van 'onze' pups en de gedragsexperimenten.

Graag wil ik de medewerkers en AIO's van de vakgroep Cellulaire Neurowetenschappen en Kindergeneeskunde bedanken voor hun wetenschappelijke input, de gezellige tijd tijdens congressen, in het lab, bij het imaging systeem en tijdens de lunch. In het bijzonder, wil ik Jan de Vente en Arjan Blokland bedanken voor hun wetenschappelijke ondersteuning en hun kriti- 
sche opmerkingen. Ik heb veel bewondering voor jullie onderzoek. Verder wil ik Jos Prickaerts, Monique Mulder, Dick Terwel en Arjan Scheepens bedanken voor hun interesse en begeleiding bij nieuwe uitdagingen. Dick, zonder jou was ik vermoedelijk nooit aan een promotieonderzoek begonnen. Ik wens je veel succes met je onderzoek in Leuven en ik hoop dat we in de toekomst nog eens samen kunnen werken. Beste Arjan, we hebben menig uurtje gevuld met kletsen over onze onderzoeksideeën, onze toekomstplannen en reisplannen. Ik wil je bedanken voor het corrigeren van mijn Engels en de gezellige tijd tijdens de congressen. Ik hoop dat we snel samen kumnen gaan duiken in Nieuw Zeeland.

Dank aan mijn kamergenootje Wilma van Staveren en collega Gunter Kenis. We zaten in hetzelfde schuitje. De gesprekken over de afronding van onze manuscripten en de arbeidsmarkt hebben me gesteund bij mijn zoektocht naar een nieuwe baan. Wilma, ik zal je missen als kamergenoot.

Verder wil ik Karin Ruijtembeek bedanken voor de gezelligheid tijdens de congressen. We hebben aardig wat afgereisd samen: Vlieland, Boston, Nieuw Zeeland. Veel succes met je nieuwe carriére als medical writer/clinical research associate. Tevens wil ik Twain Mulder en Eduardo Villamor bedanken voor hun gezelligheid tijdens congressen. Tamara, Marleen en Natascha wil ik bedanken voor al het geregel door de jaren heen. Bedankt dat ik bij jullie kon komen 'beppen' en stoom afblazen.

In addition, I wish to express my gratitude to Prof. A. C. Cuello. Dear Claudio, thank you for giving me the opportunity to work in your lab in Montreal for a few months. I had a great time and I learnt a lot about imaging techniques. I thank Dr. Christoph Schmitz for teaching me the principles of stereology en his thoughtful ideas and critical comments on the research designs and manuscripts. Equally, I thank Prof. W. Paschen for giving me the opportunity to work in his lab at the Max-Planck institute in Cologne.

Ook mijn vrienden in Maastricht wil ik bedanken voor hun steun. Alhoewel het vaak moeilijk te begrijpen was waarom ik 's avonds en in het weekend wilde doorwerken, hebben jullie altijd interesse in mijn werk getoond. In het bijzonder wil ik Semahat Gundogmus, Anja Klein en Paula Jansen noemen.

Lieve familie. Ma en pa, bedankt voor jullie steun en het feit dat jullie altijd voor me klaar staan. Marjo, fijn dat je mijn paranimf bent.

Lieve Pieter, bedankt voor jouw geduld en betrokkenheid gedurende de laatste 2 jaar van mijn promotie-periode. De avontuurlijke tocht door de woestijn van Azerbaijan, het klussen in Rijswijk, het houthakken in het bos, onze uren voor de brandende kachel...... deze gezamenlijke activiteiten hebben de afgelopen twee jaar heel speciaal gemaakt.

Rijswijk, 6 april 2003 